\title{
COMPARISON AND PREFERENCE: MANUAL SIGNING, PICTURE EXCHANGE, AND SPEECH-GENERATING DEVICES AS COMMUNICATION MODES FOR CHILDREN WITH DEVELOPMENTAL DISABILITIES
}

\author{
BY \\ LARAH VAN DER MEER
}

\begin{abstract}
A thesis
submitted to Victoria University of Wellington

in fulfilment of the requirements for the degree of

Doctor of Philosophy
\end{abstract}

Victoria University of Wellington

2012 


\section{THESIS HIGHLIGHTS}

- Twelve children (across three studies utilising single-case designs) with autism spectrum disorder (ASD) and other developmental disabilities (DD) were taught to request preferred snacks and/or toys using an $\mathrm{iPod} 囚 / \mathrm{iPad}(\mathrm{B}$-based speech-generating device (SGD), picture exchange (PE), and manual signing (MS)

- Preference for using the SGD versus PE versus MS was assessed before, during, and after acquisition training

- The intervention procedures appeared to result in the acquisition of at least one of the communication options for all 12 children

- Eight children demonstrated a preference for using the SGD, three children showed a preference for $\mathrm{PE}$, and one child appeared to show a preference for MS

- Acquisition and maintenance of requesting skills was better with the preferred communication option 


\section{ABSTRACT}

Children with autism spectrum disorder (ASD) and related developmental disabilities (DD), who do not speak or have very limited spoken language, are often candidates for augmentative and alternative communication (AAC). Three common modes of AAC are manual signing (MS), picture exchange (PE), and speech-generating devices (SGDs). Studies suggest that children with ASD and other DD might demonstrate comparable proficiency in learning to use each of these communication systems. Because there may not be one single mode of AAC that appears to be most readily taught to, and learnt by, these children, decisions regarding which AAC mode to implement might need to be based upon an assessment of the individual's preferences for using different AAC systems. Giving students the opportunity to self-select their most preferred AAC mode could also be viewed as one way of promoting self-determination in AAC intervention and this in turn might significantly influence progress in learning to communicate and maintenance of newly acquired AAC skills.

The three empirical intervention studies that are presented in the present thesis each made use of systematic instructional procedures, based upon the principles of applied behaviour analysis (ABA), to teach children with ASD and other DD to request (mand) preferred items using MS, PE, and SGD. A key aspect of the studies was the assessment of each child's preference for using one of the three AAC modes via a structured choice-making protocol. This choice-making protocol was designed to determine whether children made greater progress, showed increased communication ability, and continued to use $A A C$ during follow-up sessions when their preferences for different $A A C$ options were assessed and incorporated into the intervention process.

Utilising single-case research methodology (combined multiple-baseline and alternating-treatments designs), a total of 12 participants ( 9 boys, 3 girls, aged 4 to 13 years) received interventions that were designed to compare acquisition, maintenance, and preference for MS, PE, and SGD. Overall, the results of the three studies demonstrated that seven of the 12 (58\%) participants learnt to use, and reached criterion, for use of each AAC option. Five participants did not reach criterion for MS. Two participants did not reach criterion for $P E$ and one of these participants did not reach criterion for SGD. Eight of the 12 (67\%) 
participants appeared to show a preference for using the SGD. Preference for using PE was demonstrated by three out of eight (38\%) participants (PE was only assessed in Studies 2 and 3). Finally, one out of the 12 (8\%) participants appeared to show a preference for using MS.

These data suggest greater proficiency and better maintenance when children used their preferred communication option. These findings highlight some potentially positive effects of enabling some degree of self-determination with respect to aspects of the use of AAC systems in communication intervention. Given the encouraging intervention outcomes for the present series of three studies, future research and practice should continue to seek ways of increasing opportunities for self-determination in AAC and related interventions for individuals with ASD/DD. 


\section{PUBLICATIONS ARISING FROM, AND RELATED TO, THE PRESENT THESIS}

Rispoli, M., Franco, J., van der Meer, L., Lang, R., \& Carmargo, S. (2010). The use of speech generating devices in communication interventions for individuals with developmental disabilities: A review of the literature. Developmental Neurorehabilitation, 13, 276-293.

van der Meer, L., Didden, R., Sutherland, D., O'Reilly, M., Lancioni, G., \& Sigafoos, J. (2012). Comparing three augmentative and alternative communication modes for children with developmental disabilities. Journal of Developmental and Physical Disabilities, 24, 451-468.

van der Meer, L., Kagohara, D., Achmadi, D., O'Reilly, M., Lancioni, G., Sutherland, D., \& Sigafoos, J. (2012). Speech-generating devices versus manual signing for children with developmental disabilities. Research in Developmental Disabilities, 33, 1658-1669.

van der Meer, L., \& Rispoli, M. (2010). Communication interventions involving speech-generating devices for children with autism: A review of the literature. Developmental Neurorehabilitation, 13, 294-306.

van der Meer, L., Sigafoos, J., O'Reilly, M., \& Lancioni, G. (2011). Assessing preferences for AAC options in communication interventions for individuals with developmental disabilities: A review of the literature. Research in Developmental Disabilities, 32, 1422-1431.

van der Meer, L., Sutherland, D., O'Reilly, M., Lancioni, G., \& Sigafoos, J. (2012). A further comparison of manual signing, picture exchange, and speechgenerating devices as communication modes for children with autism spectrum disorders. Research in Autism Spectrum Disorders, 6, 1247 1257. 


\section{ACKNOWLEDGEMENTS}

The present thesis would not have been possible without the constant guidance and support from my primary supervisor, Professor Jeff Sigafoos. Thank you. Special thanks also to my secondary supervisor, Associate Professor Matt McCrudden, for your continued support over the past three years and input on the final thesis draft.

Thank you very much to my cohort group, fellow postgraduate students, and most of all Tara Evans, for not only sharing ideas and providing feedback and support, but (importantly) for the never ending supply of home baking and much needed breaks from all the hard work.

Special recognition also needs to be given to the children, families, and teachers who participated in this research. It has been a pleasure working with you.

I would like to thank Dr. Dean Sutherland, Dr. Robert Didden, Dr. Mark O’Reilly, Dr. Guilio Lancioni, Dr. Debora Kagohara, as well as Donna Achmadi and Laura Roche for helping implement the studies presented in this thesis and manuscripts that resulted from this work.

Support for this research was provided from the New Zealand Government through the Marsden Fund Council, administered by the Royal Society of New Zealand; and by Victoria University of Wellington, Vice-Chancellor's Strategic Research Scholarship.

Last but not least thanks go to my family: my parents Edith and Ton, my brothers Luke and Steven, and my sister Sanne, for your endless faith, love, and support. Finally, to my friends (you know who you are if you are reading this), you kept me going. 


\section{DECLARATION BY THE AUTHOR}

The present thesis consists of the author's original work conducted for this $\mathrm{PhD}$ under the supervision of Victoria University of Wellington. No part of it has been previously submitted for a degree or diploma. Work by other authors has been duly referenced in text and contribution by others in jointly-authored work has been clearly stated.

Ethical approval for Study 1 included in this thesis was part of a broader research project: Enhancing the communication, leisure, and social skills for children with developmental disabilities. Ethical approval for Study 2 was gained specifically for replicating Study 1 in The Netherlands: Comparing speechgenerating devices and manual signing as communication aids for children with autism. Ethical approval for Study 3 was also part of a different broader research project: Enhancing communication intervention for children with autism. The ethical approval letters for each of these studies are included in Appendix A.

Input and feedback were received from the author's primary supervisor, Professor Jeff Sigafoos, and other members of a cohort team supervised and employed by Sigafoos. However, the studies presented in the current thesis were soley the independent work of the author, with conceptual input from Sigafoos, several international mentors, and peers. The author designed and conducted three studies in consultation with several international mentors and peers.

Sigafoos also provided advice with respect to data analysis and interpretation of results in all three studies and edited drafts of the subsequent articles, as well as the chapters in this thesis. Associate Professor Matt McCrudden (co-supervisor) edited drafts of the thesis chapters.

The author reports no conflicts of interests. The author alone takes sole responsibility for the content and writing of this thesis. 
TABLE OF CONTENTS

\begin{tabular}{|c|}
\hline 115 \\
\hline 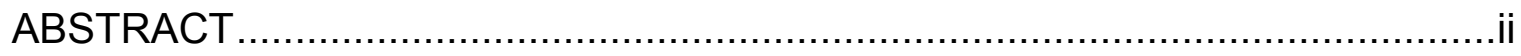 \\
\hline PUBLICATIONS ARISING FROM, AND RELATED TO, THE PRESENT THESIS \\
\hline , \\
\hline ACKNOWLEDGEMENTS ..................... \\
\hline DECLARATION BY THE AUTHOR ... \\
\hline TABLE OF CONTENTS ... \\
\hline LIST OF TABLES... \\
\hline LIST OF FIGURES \\
\hline LIST OF APPENDICES \\
\hline 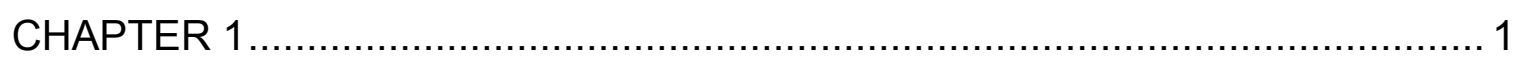 \\
\hline |INTRODUCTION \\
\hline 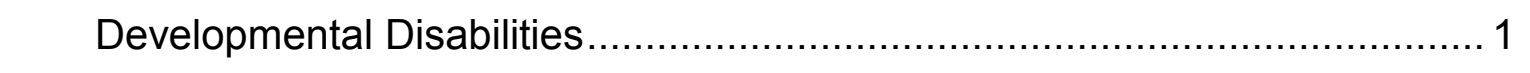 \\
\hline ASD \\
\hline 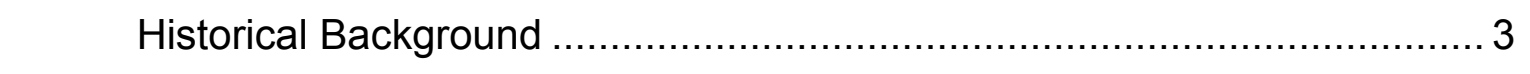 \\
\hline Diagnostic Criteria and Defining Characteristics ……........................... 4 \\
\hline Explanations, Causes, and Risk Factors ........................................... 10 \\
\hline 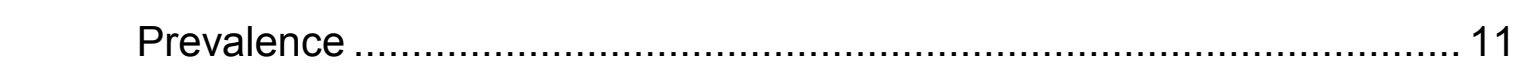 \\
\hline 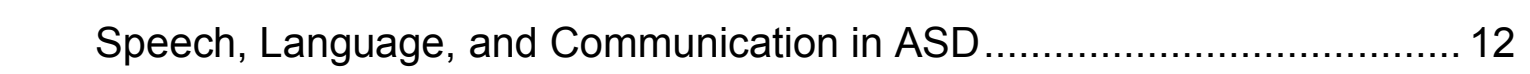 \\
\hline 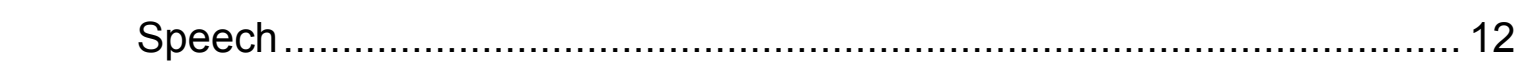 \\
\hline 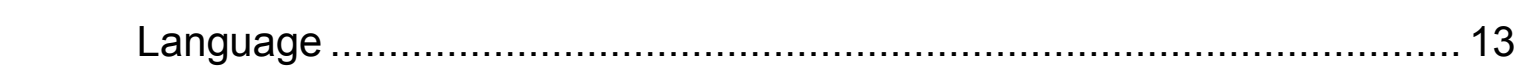 \\
\hline Communication ............. \\
\hline Communication Impairment . \\
\hline Communication Interventions in ASD... \\
\hline CHAPTER 2. \\
\hline AAC INTERVENTIONS FOR INDIVIDUALS WITH ASD AND DD ...............23 \\
\hline What is AAC? \\
\hline 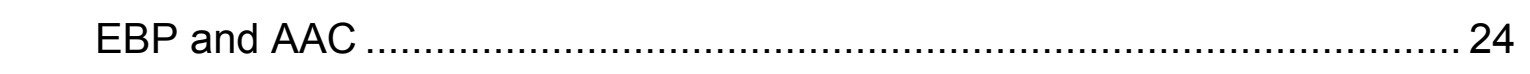 \\
\hline 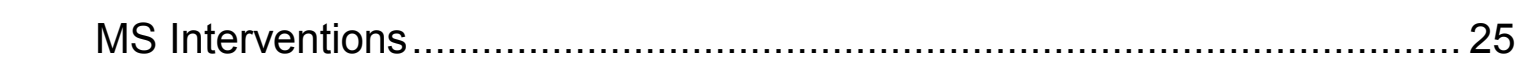 \\
\hline 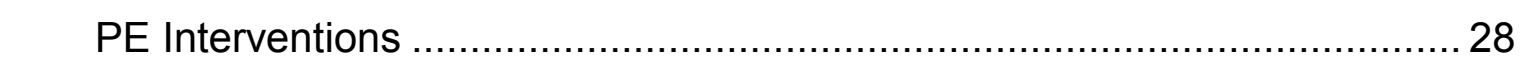 \\
\hline 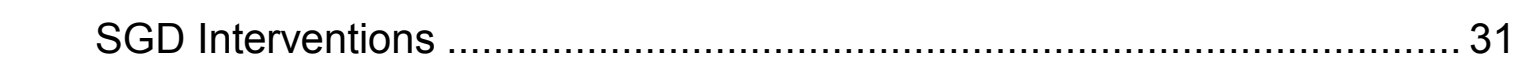 \\
\hline 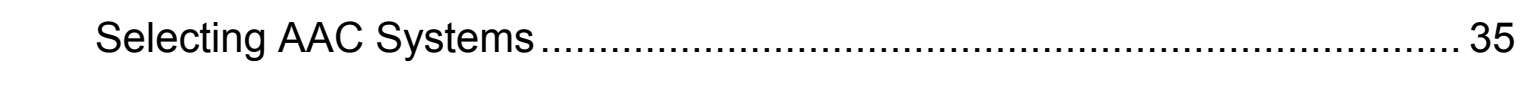 \\
\hline
\end{tabular}




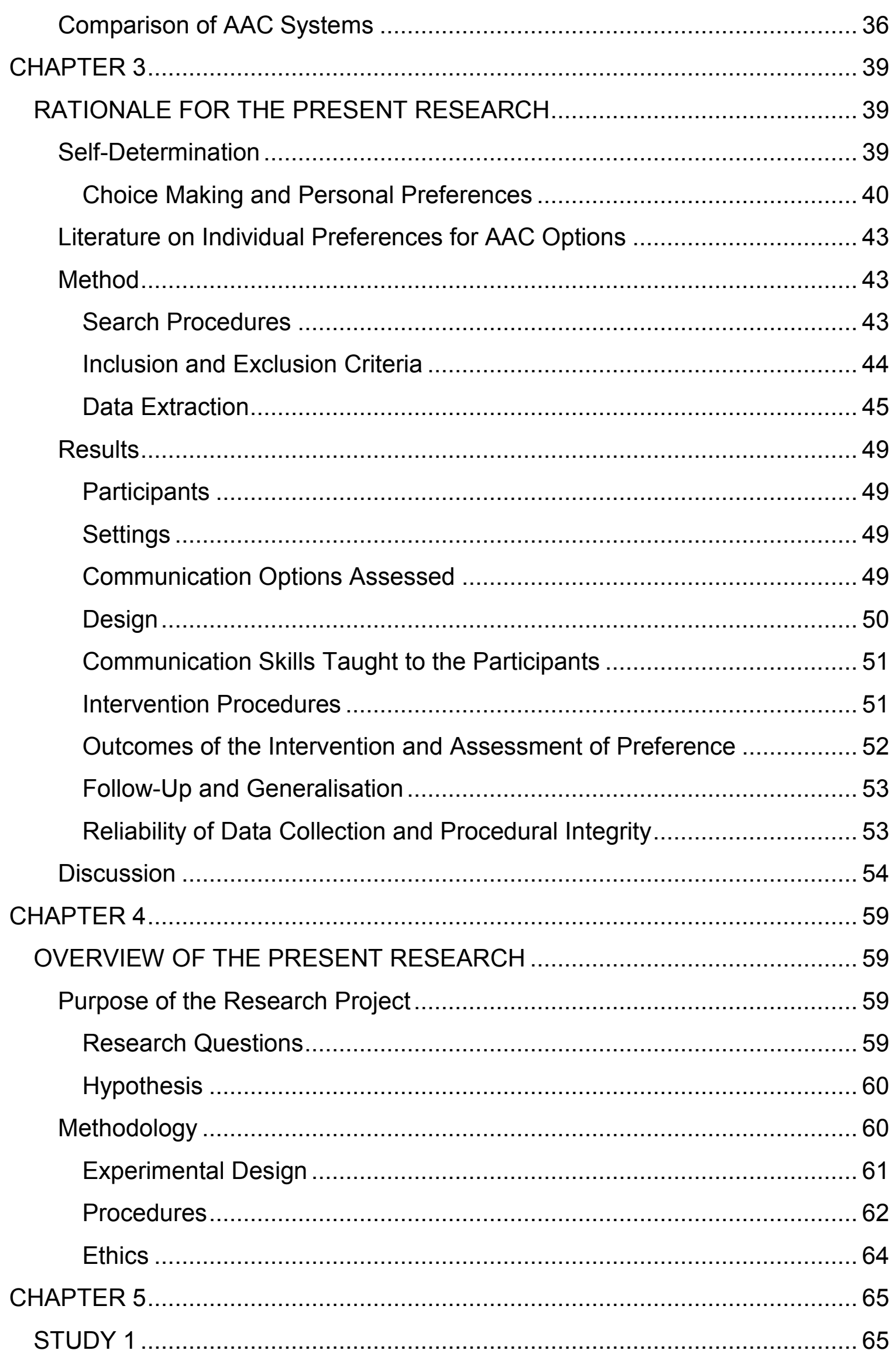




\begin{tabular}{|c|c|}
\hline & peech-Generating Devices versus Manual Signing in Children with \\
\hline & 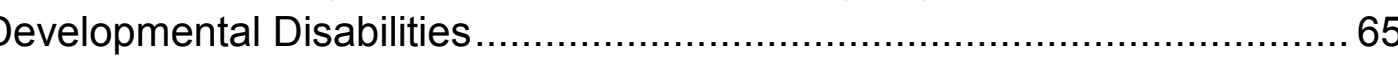 \\
\hline & 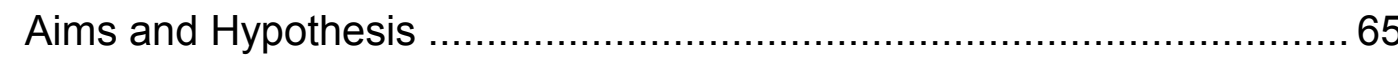 \\
\hline & Method.. \\
\hline & Participants .................................. \\
\hline & 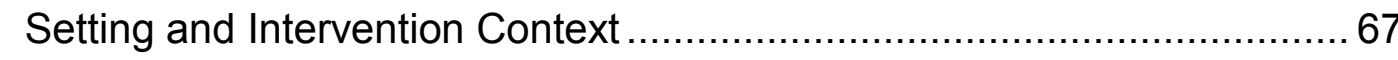 \\
\hline & 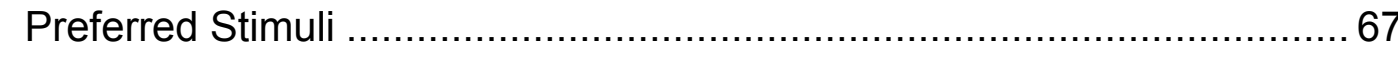 \\
\hline & 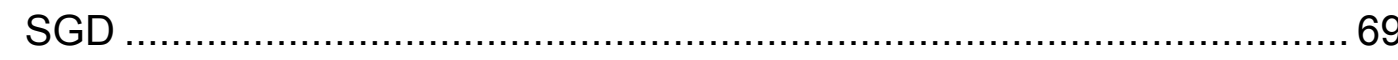 \\
\hline & MS \\
\hline & 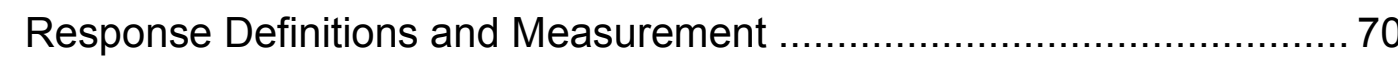 \\
\hline & 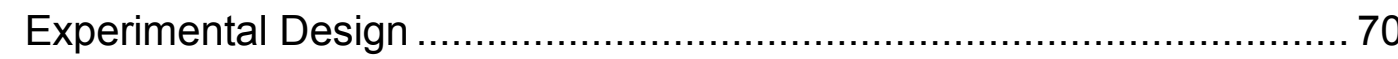 \\
\hline & Session Schedule ................................................................... 71 \\
\hline & 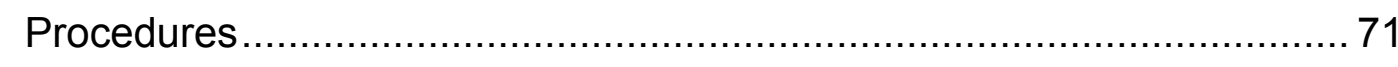 \\
\hline & 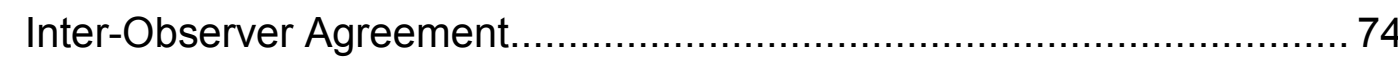 \\
\hline & 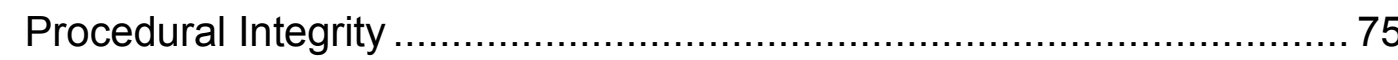 \\
\hline & Results...... \\
\hline & David ........ \\
\hline & 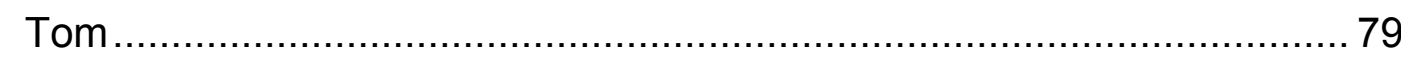 \\
\hline & 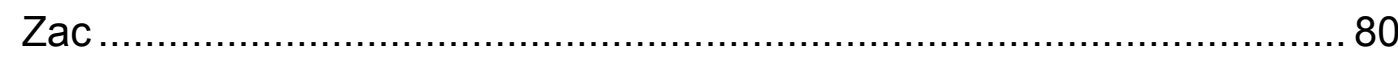 \\
\hline & 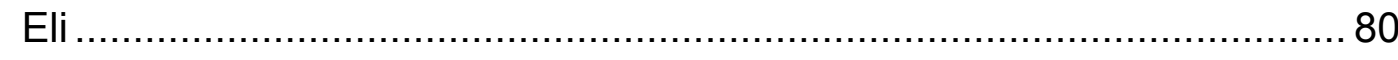 \\
\hline & iscussion .............................. \\
\hline CHAP & 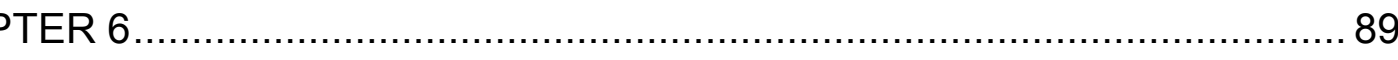 \\
\hline STL & 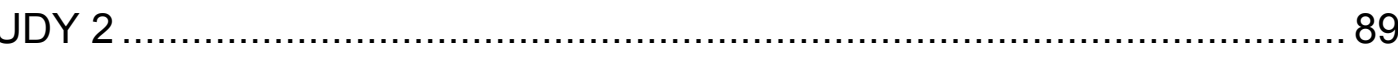 \\
\hline & Oomparing Three Augmentative and Alternative Communication Modes for \\
\hline & hildren with Developmental Disabilities ........................................... 89 \\
\hline & 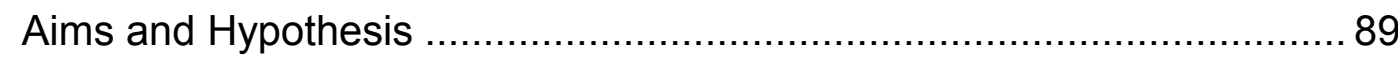 \\
\hline & 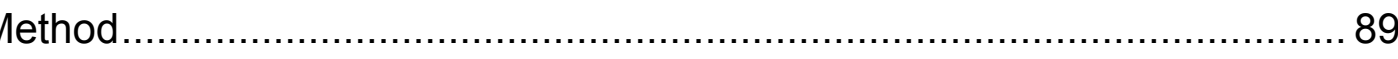 \\
\hline & 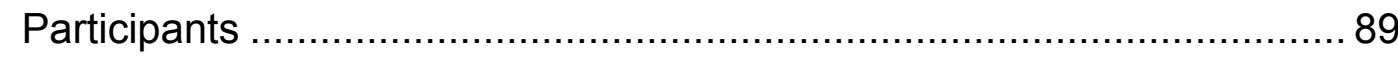 \\
\hline & 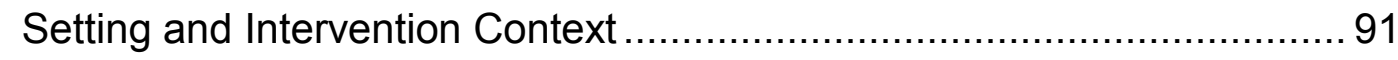 \\
\hline & 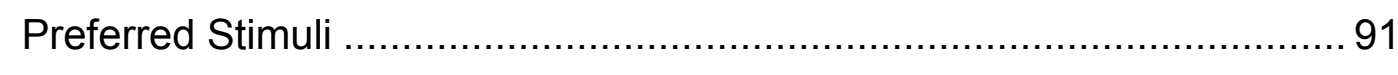 \\
\hline & 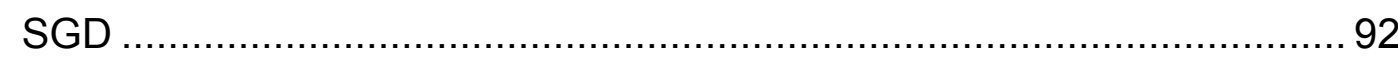 \\
\hline & 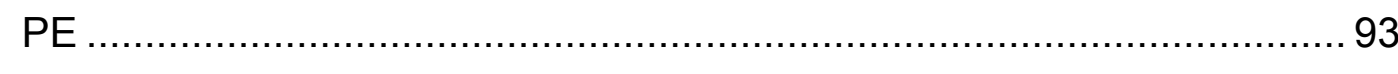 \\
\hline & MS \\
\hline & Response Definitions and Measurement ... \\
\hline
\end{tabular}




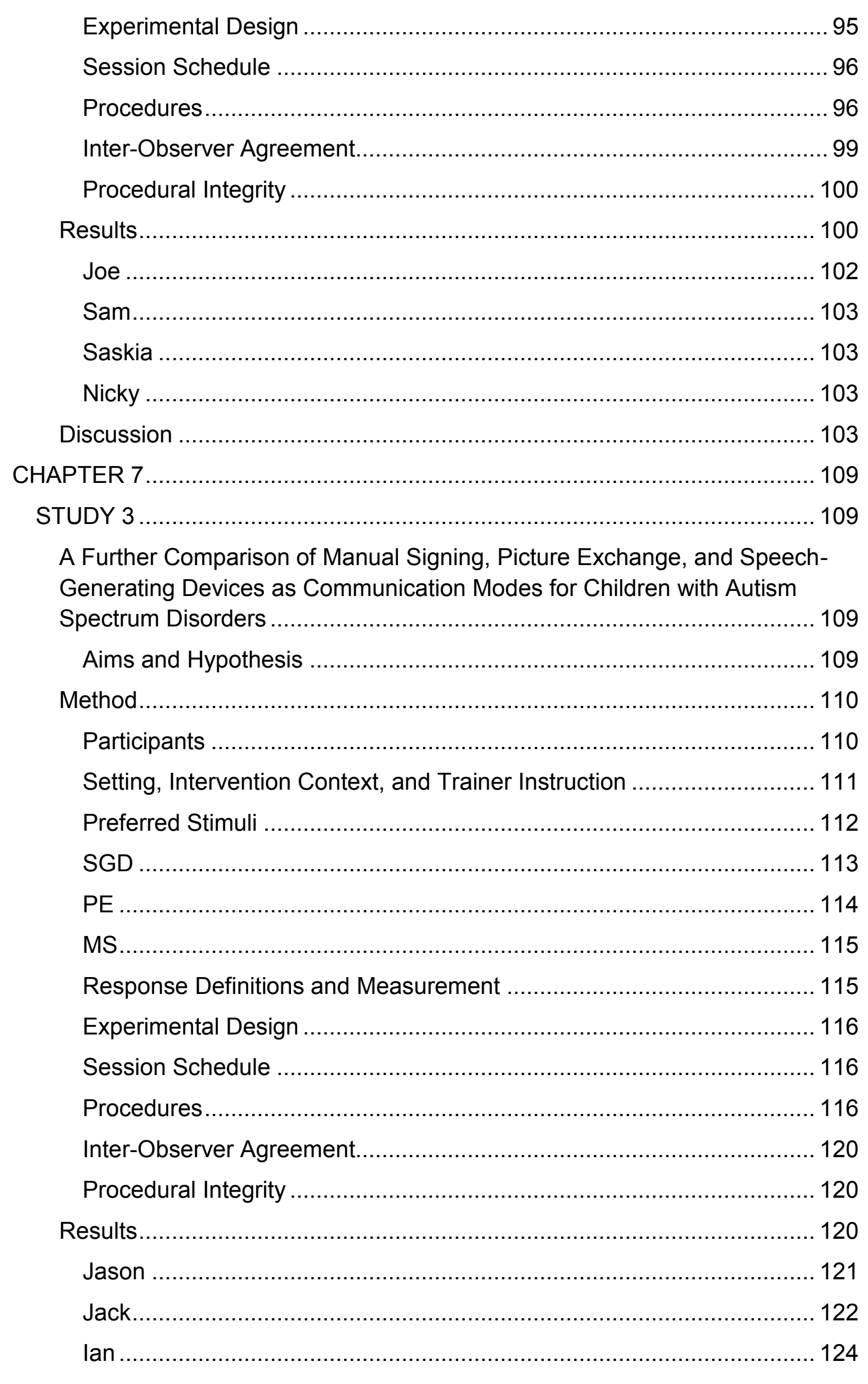




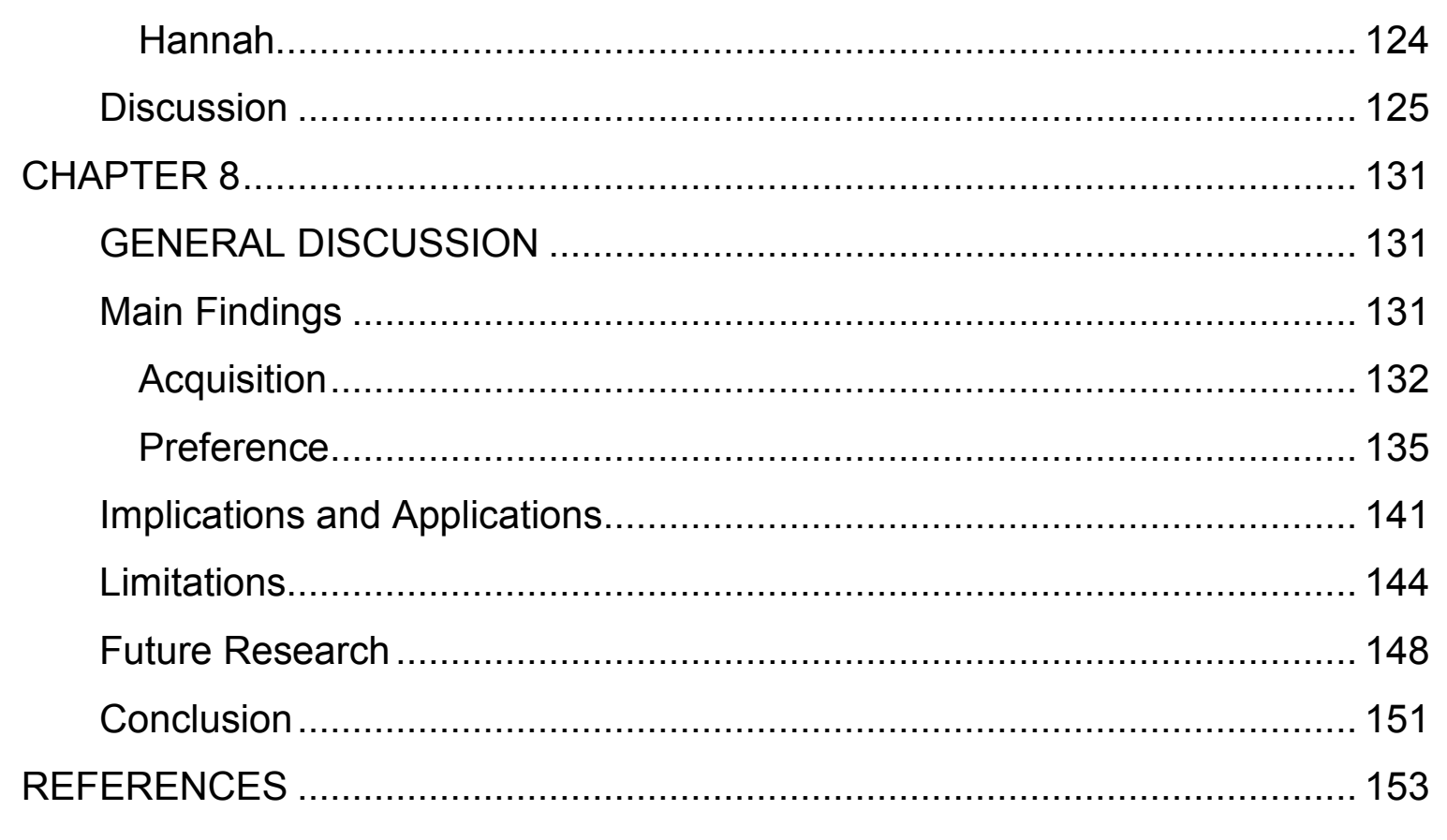




\section{LIST OF TABLES}

Table 1.1. Distinction in Classifications for DD between ICD-10 and DSM-IV-TR 2

Table 1.2. DSM-IV-TR Diagnostic Criteria for Autistic Disorder .......................... 7

Table 1.3. DSM-5 Revised Diagnostic Criteria for Autism Spectrum Disorder...... 9

Table 1.4. Language and Communication Impairments in Individuals with ASD 15

Table 1.5. Verbal Operants and Controlling Variables................................ 21

Table 2.1. Summary and Comparison of Three Common AAC Systems (MS, PE,

and SGD)................................................................................. 26

Table 3.1. Studies Assessing Preference for Various Communication Options.. 46

Table 4.1. Structured Choice-Making Protocol .........................................64

Table 5.1. NAP Effect Sizes from Baseline to Intervention for the SGD and MS

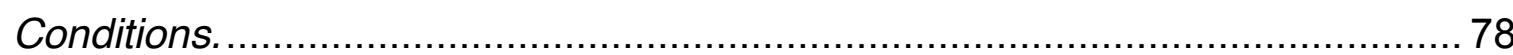

Table 6.1. Baseline to intervention NAP Effect Sizes for SGD, PE, and MS for each Participant ........................................................................ 102

Table 7.1. Percentage of Selection/Times Offered for SGD, PE, and MS During the Pre-Baseline AAC Preference Assessments. ........................................ 121

Table 7.2. Baseline to Intervention NAP Effect Sizes for SGD, PE, and MS for

each Participant. ............................................................................. 124 


\section{LIST OF FIGURES}

Figure 5.1. Photograph of the iPod Touch ${ }^{\circledR}$ inside the iMainGo ${ }^{\circledR} 2$ Speaker Case with the Visual Display of the Graphic Symbols for SOCIAL INTERACTION, SNACKS, and PLAY for Study 1. 69

Figure 5.2. Photograph of the Trainer Making the Signs for PLAY and EAT to

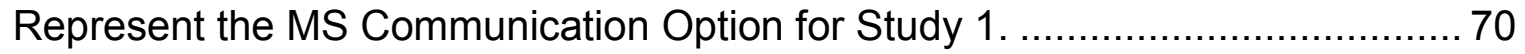
Figure 5.3. Results from the AAC Preference Assessments Depicting the Number of Times each Communication Option (SGD and MS) was Chosen across Phases and Participants. 76

Figure 5.4. Percentage of Correct Requests with SGD and MS across Sessions for each Participant. Figure 6.1. Photograph of the iPod Touch ${ }^{\circledR}$ inside the iMainGo ${ }^{\circledR} 2$ Speaker Case with the Visual Display of the Graphic Symbols for PLAY and SNACKS for Study

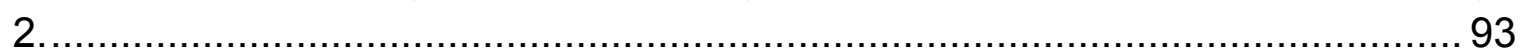

Figure 6.2. Photograph of the PE Communication Option with Graphic Symbols for I WANT, TO PLAY, and SOMETHING TO EAT for Study $2 . \ldots \ldots \ldots \ldots \ldots \ldots . . . \ldots 94$ Figure 6.3. Photograph of the Trainer Making the Signs for EAT and PLAY to Represent the MS Communication Option for Study 2.

Figure 6.4. Results from the AAC Preference Assessments Depicting the Number of Times each Communication Option (SGD and MS) was Chosen across

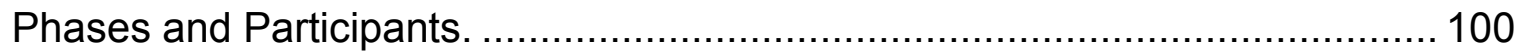
Figure 6.5. Percentage of Correct Requests with SGD and MS across Sessions for each Participant. 101

Figure 7.1. Photograph of the iPod Touch ${ }^{\circledR}$ Inside the iMainGo ${ }^{\circledR} 2$ Speaker Case with the Visual Display of the Graphic Symbols of each Preferred Item Targeted

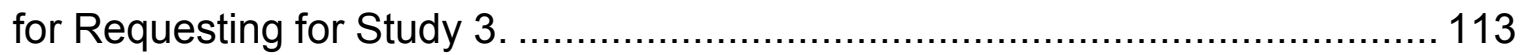
Figure 7.2. Photo of the $\mathrm{iPad}^{\circledR}$ with the Visual Display of the Graphic Symbols of

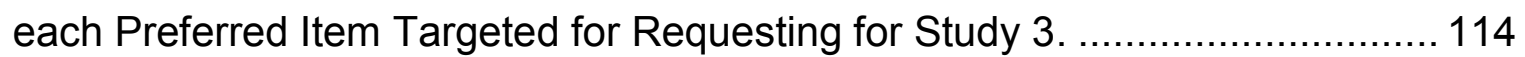
Figure 7.4. Photograph of the Makaton Signs of each Preferred Item Targeted for Requesting to Represent the MS Communication Option for Study 3............115 Figure 7.5. Results from the AAC Preference Assessments Depicting the Number of Times each Communication Option (SGD, PE, and MS) was Chosen Across

Phases and Participants. .................................................................. 122 Figure 7.6. Percentage of Correct Requests with SGD, PE, and MS across

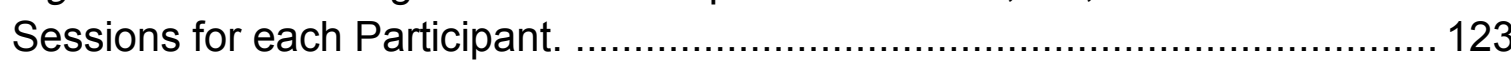




\section{LIST OF APPENDICES}

Appendix A: Ethics approval letters for each of the three studies 179 Appendix B: van der Meer, L., \& Rispoli, M. (2010). Communication interventions involving speech-generating devices for children with autism: A review of the literature. Developmental Neurorehabilitation, 13, 294-306 185 Appendix C: Rispoli, M., Franco, J., van der Meer, L., Lang, R., \& Carmargo, S. (2010). The use of speech generating devices in communication interventions for individuals with developmental disabilities: A review of the literature.

Developmental Neurorehabilitation, 13, 276-293 199

Appendix D: van der Meer, L., Sigafoos, J., O'Reilly, M., \& Lancioni, G. (2011). Assessing preferences for AAC options in communication interventions for individuals with developmental disabilities: A review of the literature. Research in Developmental Disabilities, 32, 1422-1431 219 Appendix E: van der Meer, L., Kagohara, D., Achmadi, D., O'Reilly, M., Lancioni, G., Sutherland, D., \& Sigafoos, J. (2012). Speech-generating devices versus manual signing for children with developmental disabilities. Research in Developmental Disabilities, 33, 1658-1669. 231 Appendix F: van der Meer, L., Didden, R., Sutherland, D., O'Reilly, M., Lancioni, G., \& Sigafoos, J. (2012). Comparing three augmentative and alternative communication modes for children with developmental disabilities. Journal of Developmental and Physical Disabilities, 24, 451-468. 245 Appendix G: van der Meer, L., Sutherland, D., O'Reilly, M., Lancioni, G., \& Sigafoos, J. (2012). A further comparison of manual signing, picture exchange, and speech-generating devices as communication modes for children with autism spectrum disorders. Research in Autism Spectrum Disorders, 6, 1247-1257 ... 265 


\section{CHAPTER 1}

\section{INTRODUCTION}

\section{Developmental Disabilities}

The term developmental disabilities (DD) refers to a group of conditions characterised by mental and/or physical impairment, leading to functional limitations in major life activities, such as language, learning, mobility, self-help, and independent living (Accardo \& Whitman, 1996; Centers for Disease Control and Prevention, 2011). This group includes the more specific conditions of autism, and autism spectrum disorder (ASD), cerebal palsy, and mental retardation (now commonly referred to as intellectual disability). Odom, Horner, Snell, and Blacher (2007) described DD as: "a set of abilities and characteristics that vary from the norm in the limitations they impose on independent participation and acceptance in society. The condition of developmental disabilities is developmental in the sense that delays, disorders, or impairments exist within traditionally conceived developmental domains such as cognitive, communication, social, or motor abilities and appear in the "developmental period," which is usually characterised as before 22 years of age" (p. 4).

As such DD is a summative descriptor for individuals that share common characteristics. However, precise terms, classifications, and diagnostic criteria are important for the identification of causes, prevalence, and intervention. Diagnostic classification systems have been established that include DD (Odom et al., 2007). While definitions can vary depending on the source, this thesis will summarise information from two of the most prominent systems: the International Classification of Diseases, now in its $10^{\text {th }}$ edition (ICD-10; World Health Organization, 1992) and the Diagnostic and Statistical Manual of Mental Disorders, now in its fourth edition with a text revision (DSM-IV-TR; American Psychiatric Association, 2000). Both the ICD-10 and DSM-IV-TR do not have a general classification for DD. Instead, they have specific criteria and guidelines for intellectual disability and pervasive developmental disorder (PDD). PDD is a summary diagnosis that contains specific criteria for autistic disorder, Rett's disorder, childhood disintegrative disorder, Asperger's disorder, and pervasive developmental disorder not otherwise specified (PDD-NOS; American Psychiatric 
Association, 2000). See Table 1.1 for a comprehensive comparison of the classifications for DD between the ICD-10 and DSM-IV-TR.

Table 1.1. Distinction in Classifications for DD between ICD-10 and DSM-IV-TR

\begin{tabular}{|c|c|}
\hline ICD-10 & DSM-IV-TR \\
\hline $\begin{array}{l}\text { F80-F89 Disorders of psychological } \\
\text { development } \\
\text { F80 Specific developmental disorders of } \\
\text { speech and language } \\
\text { F81 Specific developmental disorders of } \\
\text { scholastic skills } \\
\text { F82 Specific developmental disorder of } \\
\text { motor function } \\
\text { F83 Mixed specific developmental } \\
\text { disorders } \\
\text { F84 Pervasive developmental } \\
\text { disorders } \\
\text { F84.0 Childhood autism } \\
\text { F84.1 Atypical autism } \\
\text { F84.2 Rett's syndrome } \\
\text { F84.3 Other childhood } \\
\text { disintegrative disorder } \\
\text { F84.4 Overactive disorder } \\
\text { associated with mental retardation and } \\
\text { stereotyped movements } \\
\text { F84.5 Asperger's syndrome } \\
\text { F84.8 Other pervasive } \\
\text { developmental disorders } \\
\text { F84.9 Pervasive developmental } \\
\text { disorders, unspecified } \\
\text { F88 Other disorders of psychological } \\
\text { development } \\
\text { F89 Unspecified disorder of psychological } \\
\text { development }\end{array}$ & $\begin{array}{l}\text { Disorders Usually First Diagnosed in } \\
\text { Infancy, Childhood, or Adolescence } \\
\text { Mental Retardation } \\
\text { Mild Mental Retardation } \\
\text { Moderate Mental Retardation } \\
\text { Severe Mental Retardation } \\
\text { Profound Mental Retardation } \\
\text { Mental Retardation, } \\
\text { Severity Unspecified } \\
\text { Learning Disorders } \\
\text { Motor Skills Disorder } \\
\text { Communication Disorders } \\
\text { Pervasive Developmental Disorders } \\
\text { 299.0 Autistic Disorder } \\
299.80 \text { Rett's Disorder } \\
299.10 \text { Childhood Disintegrative } \\
\text { Disorder } \\
299.80 \text { Asperger's Disorder } \\
299.80 \text { Pervasive Developmental } \\
\text { Disorder Not Otherwise Specified } \\
\text { (Including Atypical Autism) } \\
\text { Attention-Deficit and Disruptive } \\
\text { Behavior Disorders } \\
\text { Feeding and Eating Disorders of Infancy } \\
\text { or Early Childhood } \\
\text { Tic Disorders } \\
\text { Elimination Disorders } \\
\text { Other Disorders of Infancy, Childhood, or } \\
\text { Adolescence }\end{array}$ \\
\hline
\end{tabular}

(American Psychiatric Association, 2000;

World Health Organization, 1992)

The PDD category found in the DSM-IV-TR (American Psychiatric Association, 2000) is now commonly referred to under the term ASD (Mirenda, 2008; Odom et al., 2007; Steyn \& Couteur, 2003). The term ASD will be used in this thesis to describe all of the PDDs, while DD will be used to describe all other developmental disorders, that is intellectual disability, cerebral palsy and specific 
DD syndromes, such as Down syndrome, Prader-Willi syndrome, and Williams syndrome. However, due to the heterogeneity of the autism spectrum, a further distinction between ASD (encompassing all PDD categories) and autism will be made. Autism refers to what the DSM-IV-TR calls autistic disorder and the ICD10 (World Health Organization, 1992) calls childhood autism (also known as classic autism, infantile autism, infantile psychosis, and Kanner's syndrome). Compared to the other PDDs, autism is generally seen as the more severe of the ASDs. This thesis presents three studies focused on communication interventions for children with ASD and other DD who share many of the characteristics of ASD. Therefore, the following section will provide an overview of ASD.

\section{ASD}

\section{Historical Background}

Accounts of autism were first systematically described in the 1940s by the American psychiatrist Leo Kanner (1943) as infantile autism and independently by the Austrian paediatrician Hans Asperger (1944) as autistic psychopathy. It may appear a coincidence that both used the term autism/autistic to characterise the nature of the underlying disturbance. The term comes from the Greek word autos meaning self and was used to describe the withdrawal from social relationships with people and from the outside world into the self (Frith, 1989). The term was used by psychiatrist Eugen Bleuler in 1911 to describe the basic disturbance in schizophrenia (Frith, 1989; Gillberg, 2007). In fact early accounts of autism often described the disorder as childhood schizophrenia (Wing, 1971).

Kanner (1943) published a description of 11 children with the condition that is now recognised as autism or ASD. He proposed two criteria for autism: (a) the inability to relate to others from the beginning of life, which he termed autistic aloneness, and an inborn autistic disturbance of affective contact, in which the person's relationship with others was altogether different; and (b) an anxiously obsessive desire for the maintenance of sameness. For example, the children Kanner described often seem unable to tolerate changes in routine and absorbed themselves in repetitive, sterotyped activities, such as repeatedly flicking their fingers in front of their eyes. Kanner also described what he called islets of ability and good cognitive potentialities, including excellent rote memory and good 
visual learning abilities. Interestingly, Kanner did not include language impairment in his criteria for autism (Folstein, 2006). However, he did describe unique features of the children's speech and language development, such as echolalia, inverted pronouns, the use of words for specific, literal, inflexible meaning, and generally a use of language that appeared self-stimulatory, rather than for communicating with another person.

Asperger (1944) described a group of children with remarkably similar characteristics to those reported by Kanner (1943), particularly in terms of social interactions, stereotypical and repetitive behaviour patterns, and narrow interests. Unlike Kanner's descriptions, however, the children described by Kanner were reported to have better language skills (including vocabulary, grammar, syntax, and morphology). However, these children did have unusual use of prosody and pragmatics such as peculiar vocal intonation, pedantic speech, and the use of long monologues that did not allow for reciprocal communication (Attwood, 2008). Wing translated Asperger's description into English in the 1980s and coined the term Asperger syndrome to describe these children (Wing, 1981).

Although autism was only formally described in the 1940s, classic cases of the disorder seem to have been described as early as the turn of the $18^{\text {th }}$ century by John Haslam in the United Kingdom and Jean Itard in France (Gillberg, 2007). One case is that of Victor, the Wild boy of Aveyron, who was found living wild in the woods and consequently assigned to Itard's supervision. Victor displayed characteristics that resemble current diagnostic criteria for autism. Thus, some people claim Victor had autism (Frith, 1989; Wing, 1971). Following the early descriptions by Kanner (1943) and Asperger (1944) more formal descriptions of autism have appeared along with theories about its origins as outlined in the remaining sections of this chapter.

\section{Diagnostic Criteria and Defining Characteristics}

While this thesis presents diagnostic criteria and defining characteristics based primarily on the DSM-IV-TR (American Psychiatric Association, 2000) and ICD-10 (World Health Organization, 1992), Matson, Nebel-Schwalm, and Matson (2007) provide a review of the multitude of scales used to diagnose ASD, including the Autism Diagnostic Interview - Revised (ADI-R; often referred to as the gold standard for assessment scales), Autism Diagnostic Observation 
Schedule (ADOS), Checklist for Autism in Toddlers (CHAT), Autism Behavior Checklist (ABC), and Childhood Autism Rating Scales (CARS). Various of these valid instruments are used within the New Zealand context, where early identification and formal diagnosis typically by a paediatrician is recommended (Ministries of Health and Education, 2008). Accurate diagnosis of ASD is also important if research results are to contribute to a strong evidence base for interventions to improve the quality of life for individuals with ASD.

Each of these diagnostic tools charactise ASD as a spectrum disorder associated with severe and pervasive deficits in multiple areas of development and usually identified before three years of age. Gillberg (2007) explained that it was only in the 1980s that the concept of an autism spectrum was introduced by Wing and Gillberg to include a much broader range of cases. This comprised the so called high-functioning autism (Asperger's disorder) originally described by Asperger and the low functioning autism described by Kanner (Gillberg, 2007), as well as intellectual disability and associated medical conditions (e.g., Rett's syndrome, epilepsy; Folstein, 2006). The ASDs (both low and high functioning cases) are associated with a triad of impairments, specifically: (a) reciprocal social interaction, (b) communication, and (c) the presence of restricted, repetitive, and stereotyped behaviour, interests, and activities (American Psychiatric Association, 2000; Matson et al., 2007; World Health Organization, 1992).

Social interaction. ASD is associated with qualitative impairment in the development of reciprocal social interaction. This includes failure to adequately use non-verbal behaviours, such as making eye contact, facial expression, body posture, and gesture to regulate social interaction. Children demonstrate difficulties in developing peer relationships appropriate to developmental level. Older children may have an interest in friendship, but lack understanding of the conventions of social interaction. This lack of social or emotional reciprocity is evidenced by impaired or unusual response to other people's emotions, lack of modulation of behaviour according to the social context, or weak integration of social, emotional, and communicative behaviours. Deficits in the spontaneous pursuit of shared enjoyment, interests, or achievements with other people is also 
evident (American Psychiatric Association, 2000; World Health Organization, 1992).

Communication impairment. Communication impairment is also a core and defining feature of ASD. The nature of this area of impariment includes delay in, or total lack of, the development of spoken language. Children who do not develop speech may rely on prelinguistic behaviours, including pointing, reaching, eye-gazing, other facial expressions, and mainly for behavioural regulation functions, such as gaining access to preferred items or escaping from and avoiding nonpreferred stimuli (Mirenda, 2003; Rowland, 2009). Some children may also learn to engage in problem behaviour, such as aggression, tantrums, and self-injury in an attempt to communicate their wants and needs (Durand, 1993, 1999). Children who do acquire some speech still often display patterns of communication characterised by stereotyped, idiosyncratic, or repetitive use of speech, including varying forms of echolalia, and generally lacking the qualitative forms of speech communication (Weitz, Dexter, \& Moore, 1997). Children may also lack developmentally appropriate varied, spontaneous make-believe play or social imitative play (American Psychiatric Association, 2000). Because this thesis is focused on communication interventions for children with ASD and other DD more detail on this area of functioning is provided in the section on Speech, Language, and Communication in ASD.

Stereotyped and restricted behaviour. Children with ASD also typically have stereotyped and restricted patterns of behaviour, preoccupation with one narrow interest as well as parts of objects, and/or repetitive motor movement, such as rocking, hand flapping, and lining up objects (American Psychiatric Association, 2000). These behaviours have been referred to as self-stimulatory behaviour (or self-stimulation) because they are thought to provide the child with some type of automatic reinforcement in the form of olfactory, visual, auditory, kinaesthetic, or tactile stimulation (Lovaas, 2003). Children with ASD also often show an inflexible fixation on routines, rituals, rules, and other obsessivecompulsive symptoms, resulting in behavioural outbursts if such routines are broken or certain demands are made (Gillberg, 2007). Refer to Table 1.2 for a full description of DSM-IV-TR (American Psychiatric Association, 2000) diagnostic criteria for autistic disorder. 
Table 1.2. DSM-IV-TR Diagnostic Criteria for Autistic Disorder

Autistic Disorder

A. A total of six (or more) items from (1), (2), and (3), with at least two from (1), and one each from (2) and (3):

(1) qualitative impairment in social interaction, as manifested by at least two of the following:

(a) marked impairment in the use of multiple nonverbal behaviours such as eyeto-eye gaze, facial expression, body postures, and gestures to regulate social interaction

(b) failure to develop peer relationships appropriate to developmental level

(c) a lack of spontaneous seeking to share enjoyment, interests, or achievements with other people (e.g., by a lack of showing, bringing, or pointing out objects of interest)

(d) lack of social or emotional reciprocity

(2) qualitative impairments in communication as manifested by at least one of the following:

(a) delay in, or total lack of, the development of spoken language (not accompanied by an attempt to compensate through alternative modes of communication such as gesture or mime)

(b) in individuals with adequate speech, marked impairment in the ability to initiate or sustain a conversation with others

(c) stereotyped and repetitive use of language or idiosyncratic language

(d) lack of varied, spontaneous make-believe play or social imitative play appropriate to developmental level

(3) restricted repetitive and stereotyped patterns of behaviour, interests, and activities, as manifested by at least one of the following:

(a) encompassing preoccupation with one or more stereotyped and restricted patterns of interest that is abnormal either in intensity or focus

(b) apparently inflexible adherence to specific, non-functional routines or rituals

(c) stereotyped and repetitive motor mannerisms (e.g., hand or finger flapping or twisting, or complex whole body movements)

(d) persistent preoccupation with parts of objects

B. Delays or abnormal functioning in at least one of the following areas, with onset prior to age 3 years: (1) social interaction, (2) language as used in social communication, or (3) symbolic or imaginative play.

C. The disturbance is not better accounted for by Rett's Disorder or Childhood Disintegrative Disorder.

(American Psychiatric Association, 2000)

Other areas of impairment. In addition to the three core domains needed for a diagnosis, several other areas of dysfunction have been noted in a significant proportion of individuals diagnosed with ASD. For example, sensory abnormalities are reported to be observed in approximately $90 \%$ of cases as well as reports of hyper- or hypo-activity, sleep disturbances, and gastrointestinal symptoms (Geschwind, 2009; Gillberg, 2007), although the reliability of these reports is questionable. Motor impairment, such as hypotonia, dyspraxia, and apraxia, have also been reported to be more common than previously thought 
(Geschwind, 2009; Mirenda, 2008), but again these reports need to be interpreted with caution due to the lack of well-validated assessment protocols for identifying motor impairment in cases of ASD. Children with ASD are also often reported to be at-risk for anxiety, depression, and self-injurious behaviour (Gillberg, 2007). Although Kanner (1943) did not identify intellectual disability in his original descriptions of autism, major intellectual disability (i.e., IQ $<50$ ) appears to be evident in about 25 to $50 \%$ of ASD cases (Edelson, 2006; Geschwind, 2009; Mirenda, 2008). Major intellectual disability is typically associated with more severe social and communication impairments (e.g., complete mutism) and excess behaviour, such as aggression, extreme tantrums, stereotyped movements, and self-injurious behaviour (Lovaas, 2003).

With such variation in its features, there is some concern that the ASD label is perhaps too broad and probably should not include some of the separate disorders, such as Rett syndrome and childhood disintegrative disorder, because these two conditions are not sufficiently similar to the other ASDs in terms of their diagnostic characteristics (Sigafoos, O'Reilly, \& Lancioni, 2009). Further, differential diagnosis between the various disorders within the spectrum is known to be difficult, particularly before two years of age (Matson et al., 2007). Due to the continued ambiguity surrounding the boundaries of the various disorders encompassing ASD, new diagnostic guidelines for the DSM-5 (American Psychiatric Association, 2011) have proposed dropping four of the PDD subcategories (autistic disorder, childhood disintegrative disorder, Asperger's disorder, and PDD-NOS) and instead having a one-dimensional category, officially utilising the term ASD. In addition the social and communication deficits associated with autism will be merged (see Table 1.3 for the new diagnostic guidelines proposed for the DSM-5). It is reported that these major revisions should maintain the sensitivity of the ASD diagnoses while increasing the specificity (American Psychiatric Association, 2011). However, at least one study (Worley \& Matson, 2012) has already compared symptoms of ASD between DSM-IV-TR (American Psychiatric Society, 2000) and DSM-5 criteria, with preliminary results of DSM- 5 criteria suggesting decreased sensitivity. That is, some children previously diagnosed with ASD according to DSM-IV-TR criteria would no longer be diagnosed according to DSM- 5 criteria. This has implications 
Table 1.3. DSM-5 Revised Diagnostic Criteria for Autism Spectrum Disorder

Autism Spectrum Disorder

Must meet criteria A, B, C, and D:

A. Persistent deficits in social communication and social interaction across contexts, not accounted for by general developmental delays, and manifest by all 3 of the following:

1. Deficits in social-emotional reciprocity; ranging from abnormal social approach and failure of normal back and forth conversation through reduced sharing of interests, emotions, and affect and response to total lack of initiation of social interaction,

2. Deficits in nonverbal communicative behaviors used for social interaction; ranging from poorly integrated- verbal and nonverbal communication, through abnormalities in eye contact and body-language, or deficits in understanding and use of nonverbal communication, to total lack of facial expression or gestures.

3. Deficits in developing and maintaining relationships, appropriate to developmental level (beyond those with caregivers); ranging from difficulties adjusting behavior to suit different social contexts through difficulties in sharing imaginative play and in making friends to an apparent absence of interest in people

B. Restricted, repetitive patterns of behavior, interests, or activities as manifested by at least two of the following:

1. Stereotyped or repetitive speech, motor movements, or use of objects; (such as simple motor stereotypies, echolalia, repetitive use of objects, or idiosyncratic phrases).

2. Excessive adherence to routines, ritualized patterns of verbal or nonverbal behavior, or excessive resistance to change; (such as motoric rituals, insistence on same route or food, repetitive questioning or extreme distress at small changes).

3. Highly restricted, fixated interests that are abnormal in intensity or focus; (such as strong attachment to or preoccupation with unusual objects, excessively circumscribed or perseverative interests).

4. Hyper-or hypo-reactivity to sensory input or unusual interest in sensory aspects of environment; (such as apparent indifference to pain/heat/cold, adverse response to specific sounds or textures, excessive smelling or touching of objects, fascination with lights or spinning objects).

C. Symptoms must be present in early childhood (but may not become fully manifest until social demands exceed limited capacities)

D. Symptoms together limit and impair everyday functioning.

(American Psychiatric Association, 2011)

in terms of treatment. For example, it could be that some children not holding an ASD diagnosis will still exhibit significant symptoms, that require specialist intervention, but these children might not be eligible for the funding necessary to receive the intervention. In addition, the change in diagnostic criteria associated with the move to a general ASD term could affect prevalence estimates (i.e., a narrower symptom definition may lead to decreases in prevalence figures and incidence rates). It has been argued that the lack of knowledge regarding the 
underlying pathology of ASD is the main reason for difficulties and disagreements over classification (Wing \& Attwood, 1987). The following section will review evidence regarding several proposed and possible causes of ASD.

\section{Explanations, Causes, and Risk Factors}

The cause of ASD remains unknown in $90 \%$ of cases (Steyn \& Couteur, 2003), but there is general consensus that ASD is neurological with a genetic aetiology and with multiple genes involved (Gillberg, 2007; Peeters \& Gillberg, 1999). Given the heterogeneity of symptoms within the broad autism spectrum, it is possible that there are multiple causes (Folstein, 2006; Hill \& Frith, 2004). It is also possible that different causal mechanisms are responsible for specific ASDassociated characteristics as discussed next.

Several neuropsychological theories (mind-blindness theory, empathisingsystemising (E-S) theory, executive function theory, and central coherence (CC) theory) have been proposed to explain some of the more specific characteristics of ASD. For example, deficits in empathy and social intelligence (mind-blindness theory) may have a neural basis with abnormalities found in the amygdala and left medial front cortex (Baron-Cohen, 2004). However, diverse findings have been reported in studies on brain growth and structure in ASD, with few replications until recently (Geschwind, 2009). Reviews of this literature (Filipek, 1999; Geschwind, 2009; Gillberg, 2007; Mandell, Stahmer, \& Brodkin, 2008; Peeters \& Gillberg, 1999) suggest the persons with ASD have brain overgrowth and macrocephaly, abnormalities in the frontal and anterior temporal lobes, the cerebellum, and the brain stem; as well as differences in the form, structure, and position of neurons and an imbalance of certain neurotransmitters. It may be concluded from this research that the neuroanatomy of ASD is multidimensional and affects multiple, and likely independent neural systems (Ecker et al., 2010).

These neural abnormalities, along with the behavioural, cognitive, and affective characteristics of ASD are likely to be due to genetic factors. Genetic mutations causing autism can be identified in approximately 10 to $20 \%$ of cases, but no single genetic mutation can explain more than approximately $1 \%$ of ASD cases (Geschwind, 2009). These genetic contributions are supported by familial pattern, with studies reporting a significant increase in ASD in first-degree relatives of those on the spectrum (Geschwind, 2009). For instance, 
approximately $5 \%$ of siblings of individuals with autism also have the diagnosis (American Psychiatric Association, 2000). Twin studies reveal that $60 \%$ of monozygotic twins were concordant for autism, while $0 \%$ of dizygotic twins. When the broader spectrum of related cognitive or social abnormalities was taken into consideration, $92 \%$ of monozygotic twins versus $10 \%$ of dizygotic twins were in concordance (Bailey et al., 1995). This suggests a heritability of close to $100 \%$ in cases that are not associated with specific medical disorders, such as Fagile-X syndrome and Angelman syndrome (Gillberg, 2007). Gene studies have also attempted to identify specific DNA sequence variations causing ASD.

Several environmental factors associated with ASD may interact with genetic vulnerabilities in complex ways (Losh, Sullivan, Trembath, \& Piven, 2008). For example, it appears that ASD can occur following brain damage sustained in pregnancy, around delivery, or in the postnatal period (Gillberg, 2007; Peeters \& Gillberg, 1999). Further complicating the matter, about a quarter of individuals diagnosed with ASD have an associated medical disorder with a known or probable basis (e.g., Fragile-X syndrome, Angelman syndrome, tuberous sclerosis, epilepsy, vision and/or hearing impairment), which may be the underlying cause of ASD (Peeters \& Gillberg, 1999).

\section{Prevalence}

ASD was once considered a rare condition with reported prevalence rates (in England, Wales, the United States, and Denmark) of 4 in 10,000 children (Wing, Yeates, Brierley, \& Gould, 1976). The most recent prevalence figures suggest that ASD is much more common in that it may affect 1 in every 110 children in the United States (Centers for Disease Control and Prevention, 2009). Several studies (e.g., Boyle et al., 2011; Chakrabarti \& Fombonne, 2001; Fombonne, 2003; Ritvo et al., 1989) and reviews (e.g., Campbell, Davarya, Elsabbagh, Madden, \& Fombonne, 2011; Matson \& Kozlowski, 2011; Williams, Higgins, \& Brayne, 2006) provide prevalence estimates that are consistent with this 1:110 figure. This change in prevalence estimates from 4:10,000 to 1:110 suggests large increases in the number of children being identified with ASD. Indeed, in California there has been a $500 \%$ increase in the number of children with ASD receiving special education services between 1991 and 1999 (Odom et al., 2003). It is unclear whether this increase is due to an actual rise in the 
prevalence of ASD or to other factors, such as broadening of the diagnostic criteria, better assessment, and increased awareness of ASD (Campbell et al., 2011; Matson \& Kozlowski, 2011; Ministries of Health and Education, 2008; Steyn \& Couteur, 2003).

Most of these prevalence studies focus on the broad autism spectrum, but it is important to note that the more severe autism cases seem to represent only about one third of the ASD cases overall. In addition, other conditions currently within the broad autism spectrum are rare, such as childhood disintegrative disorder and Rett syndrome (Gillberg, 2007). When considering ASD, yet exluding childhood disintegrative disorder and Rett syndrome, it is the case that boys are approximately four times more likely to be diagnosed with ASD than girls. It is thought that this ratio may be even greater in the higher functioning end of the spectrum (e.g., Asperger's syndrome; Attwood, 2008; Fombonne, 2003; Steyn \& Couteur, 2003). In New Zealand, the Disability Survey of 2006 (Statistics New Zealand, 2007) identified $5 \%$ of all children under 15 years of age as having special needs, including ASD and other DD. Although there is no information on the prevalence and incidence of ASD specifically in New Zealand, utilising conservative figures, it is estimated that 40,000 individuals might have ASD (Ministries of Health and Education, 2008).

\section{Speech, Language, and Communication in ASD}

Communication delay is a common parental concern and often the initial reason for parents seeking a referral and possible ASD diagnosis (Horovitz \& Matson, 2010; Lovaas, 2003; Paul, 2008; Short \& Schopler, 1988; Steyn \& Couteur, 2003). Depending on the source, it is estimated that up to half of children with ASD will remain nonverbal (mute) or develop only limited speech and language skills during their lives (e.g., Lovaas, 2003; National Research Council, 2001; Peeters \& Gillberg, 1999; Simpson et al., 2005; Weitz et al., 1997; Wing \& Attwood, 1987). Before describing these deficits, it may be useful to define what is meant by speech, language, and communication.

\section{Speech}

Speech refers to the sound system of language, involving articulation, fluency, and voicing (Poulson, 2009). Children with ASD can have speech problems in each of these areas. Articulation errors include omissions (e.g., ello 
for hello), substitution (e.g., weally for really), distortion (e.g., using a rolling $r$ when saying the word red), and reduplication (e.g., razzleberry instead of raspberry). Fluency problems include stuttering (speaking with hesitations, prolongations, or repetitions) and cluttering (rapid and/or irregular speech rate). Finally, voicing problems involve voice volume (high or low pitch).

\section{Language}

Poulson (2009) noted that language includes meaning (use in context), displacement (reference to objects and events that are not present), and generativity (generalisation of linguistic forms of behaviour to novel instances). Wilkinson (1998) further noted that language encompasses four interrelated components: pragmatics, semantics, phonology, and syntax. Pragmatics refers to language use within social interactions, including nonverbal social behaviours (turn-taking, eye-contact) and verbal measures of speech formality and topic selection (e.g., types of words and topics selected for conversation with a friend versus employer). Semantics (symbolic behaviour) refers to the rules surrounding word meanings and concepts, including acquisition of new words and their meanings, organisation of these concepts in memory, and production or response to these words during communication. Phonology refers to the production of speech sounds, including how we distinguish between heard words and rules surrounding pronunciation and articulation of sounds. Prosody, a subcategory of phonology, refers to the patterns of speech, including intonation, rhythm, and stress; this influences speech sounds as well as grammar. Finally, syntax refers to the structure of language and how words are put together to form sentences.

\section{Communication}

Communication is a broader term than either speech or language (Poulson, 2009). Communicative interactions typically involve at least two people, one identified as the speaker and one identified as the listener (Bondy \& Frost, 2003), although this does not negate the fact that one may be both speaker and listener in any given communicative exchange (Skinner, 1957). In prototypic communicative exchanges, the speaker makes a communicative response (e.g., makes a request, comment, or initiates a conversation) that the listener then acts upon. This communicative exchange may be intentional or non-intentional, 
involve conventional or non-conventional forms, take linguistic or non-linguistic forms, and occur through spoken or other modes (e.g., gestures, facial expression, writing; National Joint Committee for the Communicative Needs of Persons with Severe Disabilities, 1992). As with speech and language, communication can be viewed as an inherently social process in which a speaker's behaviour provides a discriminative stimulus for a listener and vice versa, regardless of the developmental level of the person or communicative forms used (Arthur-Kelly, Bochner, Center, \& Mok, 2007). Because communication can involve both speech and language, as well as other components, this broader term will be used to describe the speech, language, and communication deficits in children with ASD.

\section{Communication Impairment}

Communication impairment is necessary for a diagnosis of ASD (American Psychiatric Association, 2000; World Health Organization, 1992). The heterogeneity of expressive communication skills in ASD is a defining feature (Wilkinson, 1998), with one end of the spectrum mute and almost completely non-communicative and the other talkative and longwinded. However, differences in the comprehension (reception) of spoken language do not appear to be as apparent (Peeters \& Gillberg, 1999). While most infants initially learn new words, it is estimated that early regression of vocabulary occurs in about one third of individuals with ASD (Wilkinson, 1998), usually occurring before two years of age (Matson, Kozlowski, \& Matson, 2012). Distinctive features of those who do continue to develop expressive language include echolalia, pronoun reversals, literalness of words and phrases, sentences that appear to have no meaningful connection to the situation, and general failure to use language for communicative purposes (Kanner, 1943; Paul, 1987; Peeters \& Gillberg, 1999; Wilkinson, 1998). See Table 1.4 for a list of language impairments in ASD.

Children with ASD lack the ability to communicate information messages from one person to another; the true understanding of reciprocal language is impaired, even when understanding of words and phrases is not affected (Peeters \& Gillberg, 1999); this is true for those individuals with ASD who speak and those who are mute (Kanner, 1943). 
Table 1.4. Language and Communication Impairments in Individuals with ASD

\begin{tabular}{|c|c|c|c|}
\hline Pragmatics & Semantics & $\begin{array}{l}\text { Phonology } \\
\text { (prosody) }\end{array}$ & Syntax \\
\hline $\begin{array}{ll}\text { - } & \text { Eye-contact } \\
\text { - } & \text { Sesture } \\
\text { - } & \text { Turn-taking } \\
\text { - } & \text { Response to } \\
\text { name } \\
\text { - } & \text { Choice of topic } \\
\text { - } & \text { Relevance of } \\
\text { contributions to } \\
\text { conversations } \\
\text { - Joint-attention } \\
\text { - Perseverative } \\
\text { questioning } \\
\text { Echolalia } \\
\text { (immediate } \\
\text { and/or delayed } \\
\text { repetition of } \\
\text { words or whole } \\
\text { phrases) } \\
\text { Palilalia } \\
\text { (repeating words } \\
\text { and phrases } \\
\text { over and over } \\
\text { again, often in a } \\
\text { whisper) } \\
\text { No new } \\
\text { information/ } \\
\text { questions to } \\
\text { conversation } \\
\text { Irrelevant } \\
\text { comments into } \\
\text { conversation } \\
\text { Difficulty } \\
\text { following } \\
\text { conversational } \\
\text { topic } \\
\text { Reciprocity } \\
\text { - }\end{array}$ & 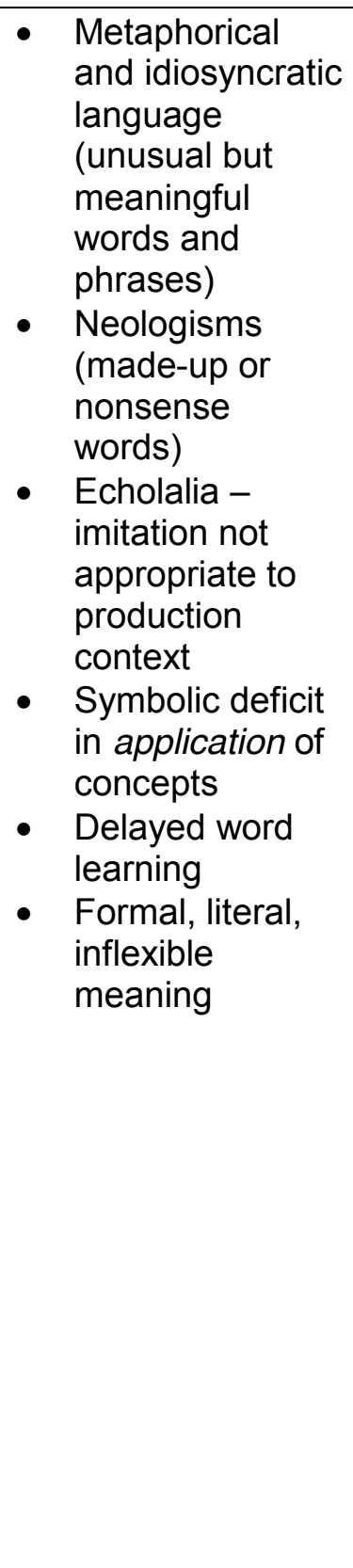 & $\begin{array}{l}\text { - Atypical } \\
\text { modulation in } \\
\text { intonation, } \\
\text { volume, rhythm } \\
\text { from monotone } \\
\text { to unusually } \\
\text { loud to singsong } \\
\text { vocal quality } \\
\text { - Echolalia can } \\
\text { retain or modify } \\
\text { original prosody } \\
\text { No improvement } \\
\text { in prosodic skills }\end{array}$ & 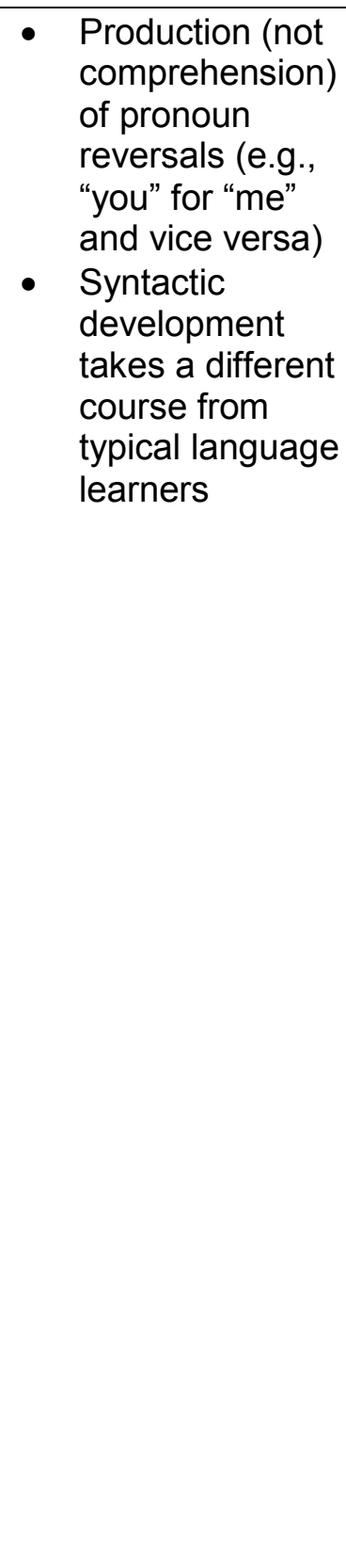 \\
\hline
\end{tabular}

Wilkinson (1998) explained that for all four language components (i.e., pragmatics, semantics, phonology, and syntax), the forms (structure) by which language is expressed are relatively unimpaired, while the functions (use/application) of language display significant impairments; that is there appears to be a "form/function dissociation" (p. 77). For example, even in nonverbal communication using the forms of eye-gaze and gestures, for 
example, can be appropriate under social or instrumental communication conditions. However, impairment is evident when the forms of language are applied to the function of joint sharing of reference or establishing joint attention. Indeed multiple sources identify a failure in the acquisition of knowledge about joint attention, as well as an inability to understand other people's cognitive state and additional socially relevant aspects of communication (Matson et al., 2012; Paul, 1987, 2008; Prizant \& Schuler, 1987; Prizant \& Wetherby, 2005; Wilkinson, 1998). It appears that communication deficits are reflective of the core characteristics of autism including social interactive deficits and cognitive style (e.g., generalisation difficulties, stimulus over-selectivity; Prizant \& Schuler, 1987). Along these lines Whitehouse, Barry, and Bishop (2008) suggested that certain combinations of autistic symptomatology may be particularly detrimental to language development. They found specific language deficits (e.g., poor nonword repetition) in children with more severe autistic traits. Although a causal relation has not been identified, data suggest a relationship between specific communication deficits and significant impairments in two or more autistic domains.

A similar pattern of impairment was evidenced in a recent study (Horovitz \& Matson, 2010), which demonstrated that toddlers with autism showed significantly more communication impairments than toddlers with PDD-NOS, who in turn showed significantly more communication impairments than toddlers with non-ASD developmental delays. Further, these deficits are evident before three years of age, despite diagnoses not typically being made until three to four years of age. Early detection of ASD and subsequent early implementation of interventions to improve communication skills would appear vital.

\section{Communication Interventions in ASD}

It would seem sensible to argue that education for children with ASD should be based upon the best available evidence of what works (Odom et al., 2003). This evidence-based practice (EBP) approach has been defined as: "the integration of best and current research evidence with clinical/educational expertise and relevant stakeholder perspectives to facilitate decisions for assessment and intervention that are deemed effective and efficient for a given direct stakeholder" (Schlosser \& Raghavendra, 2003, p. 263). Although the 
challenge to refine EBP in the education of students with disabilities is continuous (Arthur-Kelly et al., 2007), the success of numerous treatment models for individuals with ASD have been evaluated (see Odom, Boyd, Hall, \& Hume, 2010 for a review). The best available evidence currently suggests the most effective treatments are those based on the principles of applied behaviour analysis (ABA; see Matson, Benavidez, Compton, Paclawskyj, \& Baglio, 1996 for a review), such as early intensive behavioural treatment, including the application of $A B A$ practices within the UCLA Model and at the Lovaas Institute (Cohen, AmerineDickens, \& Smith, 2006; Eikeseth, Smith, Jahr, \& Eldevik, 2002; Lovaas, 1987; McEachin, Smith, \& Lovaas, 1993). Specific communication treatment models (for reviews see Brunner \& Seung, 2009; Paul, 2008) that are based on ABA, include pivotal response training (PRT, originally referred to as the natural language paradigm; Koegel \& Koegel, 2006), milieu therapy (Mancil, 2009), and functional communication training (FCT; Carr \& Durand, 1985; Mirenda, 1997).

Results from the evaluation of these ABA-based approaches provide strong scientific support for their effectiveness in teaching communication skills and other adaptive behaviours to children with ASD/DD (Brunner \& Seung, 2009; Matson et al., 1996; Odom et al., 2010; Odom et al., 2003; Smith, 1996). However, ABA has been subjected to some criticism, often surrounding limitations in the ability to produce generalisation of learnt behaviours to natural settings. Some researchers, for example, have argued that ABA-based interventions might mean that some children could become dependent on highly structured (and less natural) stimuli (e.g., response prompts) and extrinsic reinforcement (Brunner \& Seung, 2009). In light of such criticisms more naturalistic behavioural methods have been developed to address these potential limitations (Brunner \& Seung, 2009). These newer intervention models utilise empirically supported ABA-based-teaching procedures (Duker, Didden, \& Sigafoos, 2004), that are tailored to the characteristics and preferences of the child, are implemented in naturalistic contexts, and make use of advances in teaching technology (Odom et al., 2003). The aim of the research project reported in this thesis was to develop and evaluate procedures utilising such EBP-based and ABA-based procedures for enhancing the communication skills of children with ASD. 


\section{Behavioural approach to communication acquisition and}

intervention. A behavioural conceptualisation of ASD was first put forward in the 1960s by Ferster (1961), who explained that ASD might be best viewed as a disorder of behavioural excesses and deficits. These behavioural excesses and deficits might, in turn, have a neurological basis, but might also interact with environmental (reinforcement) contingencies and thus might also be ameliorated to some extent through specific, carefully programmed, constructive interactions with the environment (Green, 1996). Behavioural theory (also referred to as learning theory, operant learning theory, or operant conditioning) comprises the scientific study of behaviour involving identification of functional relations between behaviour and environment. Specifically, Skinner $(1938,1953,1957)$ developed the concept of the three-term contingency for explaining or providing a functional account of behaviour. As part of this conceptualisation, a large part of the person's behavioural repertoire, including communication (or verbal) behaviour, is shaped and maintained due to (a) the interaction between an antecedent/discriminative stimulus (event or object in the environment that set the occasion for a behaviour), (b) the response (the behaviour or target behaviour, performed in the presence of the antecedent), and (c) the resulting consequence (what happens after the response occurs). Consequences that increase behaviour are referred to as reinforcers, while consequences that decrease behaviour are defined as punishers. Skinner (1957) also introduced the term verbal behaviour, to refer to the reinforcement of a behaviour through the mediation of another person's behaviour. He suggested that verbal behaviour is mediated by the same variables that maintain nonverbal behaviour, such as motivation, discrimination, and consequences. Skinner used the term verbal operants to describe different types of verbal responses (or functional units of language) that have an effect on another person.

The mand (from the words command and demand), for example, is defined as a verbal operant in which the response is controlled by antecedent motivating variables (deprivation or aversive stimulation), later defined as establishing operations (Michael, 1988) and then refined to focus on motivational operations (Laraway, Snycerski, Michael, \& Poling, 2003), and by its characteristic consequence or reinforcing contingencies. For example, the mand 
water occurring during a state of thirst (deprivation) is reinforced by receipt of water from the listener. This type of mand is commonly referred to as requesting; communication that occurs to access items or events (positive reinforcement). Other types (or sub-classes) of mands include rejecting, advice, warning, and permission (Drasgow, Sigafoos, Halle, \& Martin, 2009; Shafer, 1994; Skinner, 1957).

The tact (from the word contact) is defined as a response that is controlled by its contact with some aspect of the environment and is reinforced by social reinforcement. For example, the tact dog occurring after seeing a dog is reinforced with praise from the listener (You're right that is a dog). Common forms of tacting include naming objects (That is a red car) and commenting on aspects of the environment (It's raining).

An intraverbal is the verbal response of a person that is controlled by the verbal stimulus of another person (e.g., one person says $d o g$ and the other responds with Labrador). Intraverbals commonly include answering questions (e.g., What is this?). They are not maintained by tangible reinforcers, but instead by generalised conditioned reinforcers (e.g., response of the other person) thereby allowing for further verbal interaction.

An echoic response is also under the control of other verbal behaviour, but its form matches the form of the verbal stimulus. It is a repeated utterance such as imitation during teaching or immediate echolalia. For example, the echoic cat occurring after hearing the word cat is reinforced with praise from the listener (Good talking!).

Last, the autoclitic refers to the listener being affected by what is suggested by the speaker, relative to the speaker's own behaviour; the autoclitic prepares the listener for what the speaker will say (e.g., use of adjectives and adverbs). See Skinner (1957) and Boisjoli and Matson (2009) as well as Table 1.5 for a detailed description of these verbal operants and their antecedent and consequent controls.

Skinner's (1957) analysis of verbal behaviour has been used as a theoretical framework for developing communication intervention programs (Poulson, 2009). That is efforts have been made to teach mands and tacts, as opposed to nouns, verbs, and other more linguistically-derived aspects of 
communication/language. However, reinforcement contingencies within the natural environment often lead to what are regarded as impure mands and partial tacts (Rehfeldt, Ziomek, \& Garcia, 2006). For example, an impure mand involves requesting desired items not only under conditions of deprivation or aversive stimulation, but also in the presence of the desired item itself. The requesting response is therefore under the control of an antecedent stimulus as well as the condition of deprivation or aversive stimulation, thereby making the responsereinforcer relation a partial tact. This is particularly the case if social reinforcement in the form of praise from the listener (e.g., Good asking! You can have the water.) is given for the response. Requesting behaviours typically taught in language acquisition interventions therefore commonly meet Skinner's (1957) definition of both mands and tacts, and thereby represent multiply controlled verbal behaviour. To avoid confusion, the present thesis will typically refer to such verbal operants simply as requesting.

In addition to using Skinner's (1957) analysis of verbal behaviour to guide the content of communication intervention programmes, the teaching procedures associated with such programmes have also been based on the basic principles of $A B A$ (otherwise referred to as behavioural intervention or behavioural treatment), such as the use of intensive one-on-one instruction, or discrete-trialtraining (Duker et al., 2004; Lovaas, 1987). An integral part of the many ABAbased interventions is the development of stimulus control (when a behaviour is emitted more often in the presence of a specific discriminative stimulus than in its absence) which can be achieved through prompting (Alberto \& Troutman, 2006; Lovaas, 2003). A prompt (an additional stimulus or response to increase the probability that the desired behaviour will occur) may be presented verbally, visually, or physically. The desired response may also be demonstrated/modelled (Alberto \& Troutman, 2006). Further specific evidence-based behavioural instructional strategies, such as graduated-guidance (Azrin \& Armstrong, 1973) and time-delay (Halle, Marshall, \& Spradlin, 1979), which are utilised in the research project presented in this thesis will be explained in Chapter 4 . 
Table 1.5. Verbal Operants and Controlling Variables

\begin{tabular}{|c|c|c|c|c|}
\hline & $\begin{array}{l}\text { Antecedent } \\
\text { Conditions }\end{array}$ & Behaviour & Consequences & Example \\
\hline Mand & $\begin{array}{l}\text { Deprivation or } \\
\text { aversive }\end{array}$ & $\begin{array}{l}\text { Verbal } \\
\text { behaviour }\end{array}$ & $\begin{array}{l}\text { Specified by } \\
\text { verbal } \\
\text { behaviour }\end{array}$ & $\begin{array}{l}\text { Say water and } \\
\text { receive water }\end{array}$ \\
\hline Tact & $\begin{array}{l}\text { Aspect of } \\
\text { current } \\
\text { environment }\end{array}$ & $\begin{array}{l}\text { Verbal } \\
\text { behaviour }\end{array}$ & $\begin{array}{l}\text { Educational } \\
\text { (verbal } \\
\text { community) and } \\
\text { social }\end{array}$ & $\begin{array}{l}\text { See soda and } \\
\text { say soda, hear } \\
\text { that's right! }\end{array}$ \\
\hline Intraverbal & $\begin{array}{l}\text { Verbal } \\
\text { behaviour of } \\
\text { another person }\end{array}$ & $\begin{array}{l}\text { Verbal } \\
\text { behaviour }\end{array}$ & Educational & $\begin{array}{l}\text { Hear dog, say } \\
\text { Labrador, and } \\
\text { then hear that's } \\
\text { right! }\end{array}$ \\
\hline Echoic & $\begin{array}{l}\text { Verbal } \\
\text { behaviour of } \\
\text { another person }\end{array}$ & $\begin{array}{l}\text { Verbal } \\
\text { behaviour that } \\
\text { is identical to } \\
\text { other's verbal } \\
\text { behaviour }\end{array}$ & Educational & $\begin{array}{l}\text { Hear ball, say } \\
\text { ball, and then } \\
\text { hear good } \\
\text { talking! }\end{array}$ \\
\hline Autoclitic & $\begin{array}{l}\text { Verbal } \\
\text { behaviour of } \\
\text { the speaker }\end{array}$ & $\begin{array}{l}\text { Verbal } \\
\text { behaviour }\end{array}$ & $\begin{array}{l}\text { Affects } \\
\text { behaviour of } \\
\text { listener relative } \\
\text { to speaker } \\
\text { (educational) }\end{array}$ & $\begin{array}{l}\text { I really want } \\
\text { chocolate, } \\
\text { informs listener } \\
\text { about an } \\
\text { aspect of } \\
\text { speaker }\end{array}$ \\
\hline
\end{tabular}

(Bondy, Tincani, \& Frost, 2004)

In its simplest form the development of new communication skills in children with ASD who lack speech can be accounted for by the basic learning processes involved in imitation and reinforcement. While several arguments outlining how imitation and reinforcement may explain language development have been widely debated (see Poulson, 2009 for a review), early behaviourally oriented interventions set out by teaching generalised imitation (imitation of responses modelled and reinforced during training as well as those that do not produce reinforcement) of oral, vocal, gross motor, and fine motor imitation (Poulson, 2009). These interventions aimed at establishing echoic behaviour (i.e., say 'ball') with few attempts at distinguishing between classes of verbal operants (Bondy et al., 2004; Shafer, 1994). Today there are well defined stages of communication development, generally distinguishing between pre-symbolic (or pre-linguistic or pre-intentional) and symbolic (or linguistic or intentional) communication (Paul, 2008; Reichle \& Brady, 2012; Rowland, 2009; Schuler \& Prizant, 1987). Drasgow and colleagues (2009) outlined a three stage model of communication development suggested by Bates and colleagues (Bates, 
Benigni, Bretherton, Camaioni, \& Volterra, 1979; Bates, Camaioni, \& Volterra, 1975): (a) perlocution (behaviour that has an effect on a listener, e.g., crying), (b) illocution (voluntary use of a signal to achieve a predictable outcome, e.g., reach out or vocalise to indicate desire for an item), and (c) locution (preverbal mands replaced with conventional verbal mands). There are now data to suggest that the mand is one of the first communicative functions to emerge in typically developing children (Drasgow et al., 2009; Reichle, 1991; Yamamoto \& Mochizuki, 1988). For this reason Reichle (1991) explained that beginning communication interventions by teaching manding is also likely to be a developmentally appropriate goal for individuals with ASD and other DD. Further, requesting access to preferred stimuli is highly functional as it enables the individual to gain reinforcement. Because the intervention directly reflects the interests of and benefits for the child a positive rapport can be established, which may increase learning and willingness to participate in later interventions aimed at teaching more socially-oriented communication. Finally, the development of requesting skills enables individuals to express their preferences and exert some degree of control over the stimuli that they access, thereby going some way towards improving their overall quality of life. (Reichle, 1991; Sigafoos \& Mirenda, 2002).

While behavioural interventions aimed at teaching spoken language have been successful, not all children appear to benefit from such intervention. That is some children may fail to acquire functional speech, even after intensive training (Lovaas, 1977; Lovaas, Simmons, Koegel, \& Stevens-Long, 1973). For these children other communication modes (e.g., picture boards or manual signs) might be utilised. These other modes are often referred to as types of augmentative and alternative communication (AAC). Schuler and Baldwin (1981) explained how non-oral communication systems may be utilised in interventions targeting functional communication skills, such as requesting. It is now widely accepted that $A A C$ systems have a role in communication interventions for children with ASD and other DD who are nonverbal or have very little spoken language (Lloyd, Fuller, \& Arvidson, 1997). Chapter 2 will provide a definition of and review the literature pertaining to $A A C$ interventions. 


\section{CHAPTER 2}

\section{AAC INTERVENTIONS FOR INDIVIDUALS WITH ASD AND DD}

\section{What is AAC?}

The term AAC refers to an area of research and clinical practice that focuses on the supplementation (i.e., augmentation) or replacement (i.e., alternative) of natural speech and/or handwriting (Beukelman \& Mirenda, 2005; Lloyd et al., 1997; Reichle, Beukelman, \& Light, 2002; Schlosser \& Sigafoos, 2006). AAC is typically considered for individuals who have either failed to acquire sufficient speech or writing or for whom speech and writing is temporarily impaired such that they do not sufficiently meet the person's everyday communication needs (Beukelman \& Mirenda, 2005).

AAC systems have been classified as either unaided or aided. Unaided $A A C$ does not rely on any auxiliary equipment; instead it involves using the individual's own body as the mode of communication. Examples include eye gaze, pointing, physically leading a communication partner's hand to an object, using conventional body language (e.g., shrugging shoulders), gestures, finger spelling, and manual signing (MS). Unaided AAC therefore generally comprises the use of body movements or sequences of co-ordinated body movements to represent an object, idea, action, or relationship. MS can be classified as one type of unaided AAC system. MS has also been defined as a topography-based (Michael, 1985) language system, in which different signs consist of different response topographies (e.g., the hand and arm movements for the sign for BALL are different to those for $D O G$ ).

Aided AAC involves the use of auxiliary equipment to transmit messages. This includes the use of graphics (traditional orthography/printed words, photographs, line drawings, or other pictographic symbols) ranging from low-tech non-electronic communication boards and picture exchange (PE) systems to high-tech electronic systems with speech output. Aided approaches can be categorised as either pointing-based in which an individual points to a symbol in order to communicate, or exchange-based in which an individual hands over a graphic symbol in exchange for an object or activity supplied by the communication partner (Sigafoos, O'Reilly, Ganz, Lancioni, \& Schlosser, 2007). 
The use of exchange-based approaches became popular with dissemination of the Picture Exchange Communication System (PECS; Bondy \& Frost, 1994; Bondy \& Frost, 2001). Similarly, the use of pointing-based systems has been increasing with the development of portable electronic speech-generating devices (SGDs), otherwise referred to as voice-output communication aids (VOCAs; Schlosser, 2003a; Schlosser \& Blischak, 2001). Both approaches can be referred to as selection-based (Michael, 1985) systems, in which all responses are topographically similar and involve the selection of a stimulus (e.g., photograph or line drawing) from an array (e.g., the pointing/exchange topography is approximately the same whether selecting a $B A L L$ or $D O G)$.

\section{EBP and AAC}

To guide EBP it is important to provide syntheses of the literature pertaining to AAC interventions for children with ASD and DD; the aims of which are to: (a) assist clinicians in their practice of improving the communication of children with ASD, and (b) identify gaps in the literature and areas in need of further research (Schlosser \& Sigafoos, 2009). With these goals in mind, various narrative and systematic literature reviews, as well as meta-analyses, of communication interventions have been undertaken for studies involving individuals with ASD (Brunner \& Seung, 2009; Ganz et al., 2011; Mirenda, 2001, 2003; Nunes, 2008) and DD (Schlosser \& Lee, 2000; Schlosser \& Sigafoos, 2006). Generally the purpose of such reviews is to answer questions in relation to the viability and efficacy of AAC intervention for individuals with ASD and DD as well as identify limitations or gaps in the existing literature.

Collectively, the results from these reviews indicated that the majority of studies have to date focused on evaluating the effectiveness of using ABA-based teaching procedures on the acquisition of one of three types of communication systems: either MS, PE, or SGDs. In addition, most studies have focused on teaching individuals to use $A A C$ to request (mand) access to preferred items (i.e., preferred foods, drinks, and toys).

While these reviews identified some methodological concerns and often limited reporting of maintenance and generalisation data, positive outcomes from the interventions were reported in most cases. With this said, it has also been highlighted that the effectiveness of any communication intervention depends on 
both the provision of an appropriate AAC system for a given individual and on the instructional procedures provided (Mirenda, 2003; Shane et al., 2011). A more detailed review of each of the three common AAC systems (MS, PE, and SGD) will now be provided. Table 2.1 also offers a summary and comparison of these three AAC options.

\section{MS Interventions}

Systematic research into the use of AAC for children with ASD and other DD was first explored in the 1970s when the use of MS was investigated by Carr, Binkoff, Kologinsky, and Eddy (1978). MS may be used to describe the use of natural sign language, such as New Zealand Sign Language (NZSL; Kennedy, 1997 ) or to the production of manual signs as a code for spoken language (Blischak, Lloyd, \& Fuller, 1997). Makaton (Makaton New Zealand/Aotearoa, 1998-99), utilised in this thesis, is a communication system that primarily uses signs from NZSL to provide a basic interactive vocabulary for children and adults with communication difficulties.

The rationale for teaching MS was based upon several assumptions. First, signs were assumed to require gross motor movement and imitation skills, which might be easier to perform, as well as requiring less verbal memory and abstract understanding than speech. Second, some signs are highly iconic, which was assumed to facilitate learning. Third, signs would appear to be more easily moulded/prompted than speech sounds. Lastly, signs can be shaped even in the absence of other social skills (Adkins \& Axelrod, 2001; Rotholz, Berkowitz, \& Burberry, 1989; Wendt, 2009). For these reasons MS was seen as a viable AAC mode that was readily taught, quick, portable, accessible, flexible, and inexpensive (Blischak, Loncke, \& Waller, 1997).

There are three major parameters to MS: location (i.e., the locations on the body or in space to distinguish signs), movement (i.e., specific movements of the hands to produce signs. For example, the direction of movement includes up, down, away from the signer, toward the signer), and hand-shape (i.e., the actual shape of the hand used to produce a sign). Location is typically the easiest and first parameter to be mastered, followed by movement. Hand-shape is generally the most difficult and last to be mastered (Blischak, Loncke, et al., 1997; Seal \& 
Table 2.1. Summary and Comparison of Three Common AAC Systems (MS, PE, and SGD)

\begin{tabular}{|c|c|c|c|c|c|c|c|}
\hline \multirow{2}{*}{$\begin{array}{l}\text { AAC } \\
\text { System }\end{array}$} & \multirow[t]{2}{*}{ Form } & \multirow[t]{2}{*}{ Description } & \multirow[t]{2}{*}{ Examples } & \multicolumn{2}{|c|}{ Advantages } & \multicolumn{2}{|c|}{ Disadvantages } \\
\hline & & & & Practical & Conceptual & Practical & Conceptual \\
\hline MS & $\begin{array}{ll}- & \text { Unaided } \\
\text { - } & \text { Topography- } \\
\text { based }\end{array}$ & $\begin{array}{l}\text { Natural sign } \\
\text { language, manual } \\
\text { signs as a code for } \\
\text { spoken language, } \\
\text { gestures (formal or } \\
\text { informal) }\end{array}$ & $\begin{array}{l}\text { NZSL (Kennedy, } \\
\text { 1997) } \\
\text { Makaton } \\
\text { (Makaton New } \\
\text { Zealand/ } \\
\text { Aotearoa, 1998- } \\
\text { 99) }\end{array}$ & $\begin{array}{ll} & \text { No auxiliary } \\
& \text { equipment } \\
\text { - } & \text { Quick } \\
\text { - } & \text { Accessible } \\
\text { - } & \text { Flexible } \\
\text { - } & \text { Inexpensive }\end{array}$ & $\begin{array}{ll} & \text { Form of } \\
\text { response } \\
\text { distinguishes } \\
\text { one verbal } \\
\text { response from } \\
\text { another }\end{array}$ & $\begin{array}{ll}\text { - } & \text { Not understood } \\
\text { by general } \\
\text { listeners } \\
\text { Reliant on } \\
\text { complex motor } \\
\text { skills and } \\
\text { imitation skills }\end{array}$ & \\
\hline PE & $\begin{array}{ll}\text { - } & \text { Aided } \\
\text { - } & \text { Selection- } \\
\text { based } \\
\text { (exchange- or } \\
\text { pointing- } \\
\text { based) } \\
\text { - } \quad \text { Low-tech } \\
\text { - } & \text { Non-electronic }\end{array}$ & $\begin{array}{l}\text { Two- or three } \\
\text { dimensional } \\
\text { representation of } \\
\text { objects or concepts } \\
\text { Real objects, line } \\
\text { drawings, } \\
\text { photographs, } \\
\text { traditional } \\
\text { orthography } \\
\text { (sets or systems) }\end{array}$ & $\begin{array}{ll}\text { - } & \text { Picture } \\
& \text { Communication } \\
\text { - } & \text { Symbols (Mayer- } \\
& \text { Johnson Co., } \\
& \text { 1994) } \\
\text { - } & \text { Blissymbolics } \\
\text { (Bliss, 1965; } \\
\text { Hehner, 1980) } \\
\text { PECS (Bondy \& } \\
\text { Frost, 1994; } \\
\text { 2001) }\end{array}$ & $\begin{array}{l}\text { - Understood by } \\
\text { - Visteners } \\
\text { Visual stimuli } \\
\text { i.e., } \\
\text { recognition- } \\
\text { based }\end{array}$ & $\begin{array}{l}\text { - Simple pointing/ } \\
\text { exchange, do } \\
\text { not need to } \\
\text { learn and } \\
\text { remember new } \\
\text { topographies }\end{array}$ & $\begin{array}{ll}\text { - } & \text { Dependent on } \\
\text { auxiliary } \\
\text { equipment } \\
\text { - } & \text { Message } \\
\text { preparation } \\
\text { - } & \text { Lack of symbol } \\
\text { diversity } \\
\text { - } \\
\text { Listener must be } \\
\text { in close proximity }\end{array}$ & $\begin{array}{l}\text { - Conditional } \\
\text { discrimination } \\
\text { and scanning }\end{array}$ \\
\hline SGD & $\begin{array}{ll}\text { - } & \text { Aided } \\
\text { - } & \text { Selection- } \\
\text { based } \\
\text { (pointing- } \\
\text { based) } \\
\text { - } \quad \text { High-tech } \\
\text { - } & \text { Electronic } \\
\text { - } & \text { Dedicated or } \\
\text { non-dedicated }\end{array}$ & $\begin{array}{l}\text { Portable electronic } \\
\text { device that displays } \\
\text { graphic symbols, } \\
\text { which represent a } \\
\text { word or phrase and } \\
\text { produce (digitised or } \\
\text { synthesised) } \\
\text { speech output }\end{array}$ & 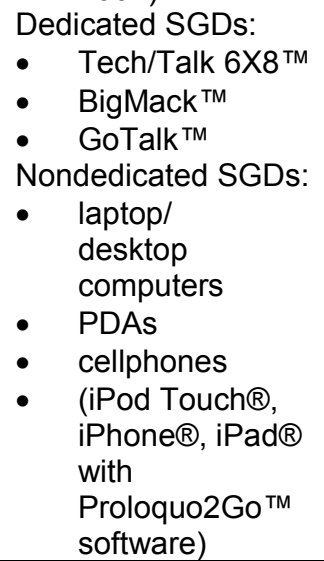 & $\begin{array}{ll}\text { - } & \text { Readily } \\
& \text { understood by } \\
& \text { all listeners } \\
\text { - } & \text { Visual stimuli } \\
& \text { i.e., } \\
& \text { recognition- } \\
& \text { based } \\
\text { Nondedicated: } \\
\text { - } \quad \text { portable } \\
\text { - } \quad \text { low cost } \\
\text { - } \quad \text { easily } \\
\text { - } \quad \text { programmed } \\
\text { socially } \\
\text { accepted }\end{array}$ & $\begin{array}{l}\text { - Simple pointing/ } \\
\text { exchange, do } \\
\text { not need to } \\
\text { learn and } \\
\text { remember new } \\
\text { topographies }\end{array}$ & $\begin{array}{l}\text { - } \quad \text { Dependent on } \\
\text { auxiliary } \\
\text { equipment } \\
\text { Dedicated: } \\
\text { - expensive } \\
\text { - cumbersome } \\
\text { - } \quad \text { time-consuming } \\
\text { to program } \\
\text { - } \quad \text { stigmatise the } \\
\text { user } \\
\text { Nondedicated: } \\
\text { - easily damaged }\end{array}$ & $\begin{array}{l}\text { - Conditional } \\
\text { discrimination } \\
\text { and scanning }\end{array}$ \\
\hline
\end{tabular}


Bonvillian, 1997). It is therefore important to consider an individual's motor skills in relation to intelligibility of sign production when considering manual signs as an AAC option. However, it appears difficult to predict the extent to which a person will find the use of MS easy and/or referable.

While MS has been taught to individuals with ASD and DD (Goldstein, 2002; Schlosser \& Wendt, 2008; Wendt, 2009), it would seem that the person's imitative skills, and in particular poor hand-motor imitation, may impact on MS learning (Tincani, 2004). Reichle and Ward (1985) point out that as children get older, signing may not be as effective for two reasons: First, they have increasing exposure to communication partners who do not understand signing. In fact, it is widely stated that the greatest disadvantage is that manual signs are not generally understood in the community at large (Blischak, Loncke, et al., 1997; Weitz et al., 1997). Second, as children learn how to read and write, a second system (printed language) becomes available (Reichle \& Ward, 1985).

In line with this idea by the mid-1980s MS was most often used in conjunction with spoken language and/or graphic symbols (Mirenda \& Erickson, 2000). When different modes (speech plus MS) are used for either (or both the) input and output of communication, the approach has been described as multimodal, total communication, or simultaneous communication (Blischak, Loncke, et al., 1997; Mirenda, 2003).

While the research on teaching MS to childen with ASD/DD has been waning in recent years (Goldstein, 2002), multiple reviews have recently synthesised findings from the existing body of literature (e.g., Goldstein, 2002; Schlosser \& Wendt, 2008; Wendt, 2009). For example, Goldstein (2002) reviewed nine studies (146 participants in total, aged 3 to 16 years) that investigated the benefits of introducing signs to children with ASD. These studies were published between 1978 and 1988, seven of which used single-case experimental designs with 1 to 10 participants and two of which were group designs. The majority of research outcomes suggested that MS alone, or as a total communication approach, resulted in faster and more complete vocabulary acquisition than speech alone. However, the research focused on teaching receptive and expressive labels (i.e., mand compliance and tacts) in response to questions, with few studies addressing what might be viewed as spontaneous 
functional communication (i.e., requesting preferred objects under relevant motivational operations).

Schlosser and Wendt (2008) reviewed 17 experimental studies, extracted from a systematic review by Wendt (2007), that assessed the acquisition and use of MS or gestures in children with ASD. Sixteen single-case and one group design (involving a total of 62 participants, aged 2 to 16 years), published between 1978 and 2004, were reviewed. The group design and two of the singlecase studies provided inconclusive (the outcomes are not a result of the intervention due to fatal flaws in the design) evidence, while the 14 remaining single-case studies provided either suggestive (the outcomes are plausible and possibly a result of the intervention due to a strong design, but inadequate interobserver agreement and/or treatment integrity), preponderant (the outcomes are plausible and more likely than not to have occurred as a result of the intervention, despite minor design flaws and adequate or better inter-observer agreement and procedural integrity), or conclusive (the outcomes are indisputably a result of the intervention based on sound design and adequate or better inter-observer agreement and procedural integrity) evidence. Most recently, Wendt (2009) supplemented and updated these previous reviews (Schlosser \& Wendt, 2008; Wendt, 2007) by synthesising a total of 21 experimental studies (18 single-case and 3 group designs) involving 130 children (1 to 16 years of age) with ASD. The studies were published between 1978 and 2007, with two single-case and two group designs ranked as providing inconclusive evidence. Across these reviews the studies that provided suggestive or conclusive evidence indicated an overall strong effect size for sign acquisition and production, as well as for other outcomes such as speech comprehension and production. The overall outcome of these studies suggested that MS was successfully taught to the majority of participants. It could therefore be viewed as a viable and generally teachable communication modality for individuals with ASD.

\section{PE Interventions}

Given that most communication partners do not understand MS-based communication (Blischak, Loncke, et al., 1997), AAC researchers broadened their evaluations beginning in the 1980s to include other modes of (aided) communication, such as PE communication (Schlosser \& Wendt, 2008). This 
interest coincided with the reported visuo-spatial strengths of individuals with ASD and the delineation of several potential benefits of using aided communication modes, such as the use of static graphic symbols (Schuler \& Baldwin, 1981). Graphic symbols used in AAC interventions are typically two- or three-dimensional representations of objects or concepts, such as real objects, miniature objects, line drawings, photographs, and traditional orthography (Beukelman \& Mirenda, 2005; Mustonen, Locke, Reichle, Solbrack, \& Lindgren, 1991). The presence of these symbols was seen as an advantage in enabling performance because it was based on recognition, rather than recall (as is required with $M S$ ).

As with MS, it is important to compare the cognitive and linguistic demands between graphic symbol sets/systems in terms of translucency/iconicity (the degree to which a symbol is similar to its referent) and transparency (the extent to which a symbol is guessable to an untrained viewer) of the symbols (De Paul \& Yoder, 1986; Johnston \& Cosbey, 2012). A general hierarchy exists where real objects are considered the most translucent and transparent, while traditional orthography is the least translucent and transparent (Johnston \& Cosbey, 2012). Unlike MS, Shafer (1993) explained that since graphic modes provide a permanent display the user does not have to 'remember' a large number of signs or symbols; recognition is simply required.

However, Michael (1985) and others (Potter \& Brown, 1997; Shafer, 1993; Sundberg, 1993) argued that selection-based modes require conditional discriminations, including identity matching or matching-to-sample skills, that may be difficult to teach to individuals with ASD and DD. Additionally, a scanning repertoire necessary to differentiate between and select symbols may be absent in children with ASD and other DD. Along these lines, Wilkinson and Mcllvane (2002) explained that the prolonged time required for message preparation, in terms of finding and selecting the correct message, as well as in creating the graphic symbols for each set/system, and the lack of symbol diversity (e.g., finding symbols to represent pronouns and adjectives) are potential problems with the use of graphic-mode AAC systems. Furthermore, Sigafoos and lacono (1993) highlighted the importance of various practical factors, such as message access (pointing- versus exchange-based), organisation and size of the symbols, 
and the expandability and portability of the set/system when considering the use of graphic-mode AAC.

Despite these potential issues with graphic-mode AAC, various such systems have been developed and evaluated for use by individuals with complex communication needs. One well-researched approach in this genre is PECS (Bondy \& Frost 1994, 2001). PECS is characterised by two main features: (a) it does not require prerequisite symbol discrimination and matching-to-sample skills usually essential to pictorial communication modes, and (b) it commences with teaching requests (mands) to enable the person to access preferred items and/or activities (Lancioni et al., 2007). PECS training initially involves the exchange of a picture for a reinforcing item. This parallels the communicative exchange of requesting that takes place in the prototypic mand scenario (Skinner, 1957) and this initial focus has been one of the major reasons for why PECS has proven to be readily taught to children with ASD/DD. Indeed, data show that PECS is often rapidly acquired and leads to self-initiated communication (Bondy \& Frost, 1994). The PECS training protocol (Frost \& Bondy, 2002) is based on Skinner's (1957) analysis of verbal behaviour and ABA-based instructional strategies (Duker et al., 2004), including capturing or creating motivational operations, response prompting, differential reinforcement, error correction, and programming for generalisation. The PECS protocol is divided into six phases in which children first learn to communicate with single pictures, then choose among two or more pictures, and finally combine pictures to produce a variety of grammatical structures, semantic relationships, and communicative functions.

The pace of publication regarding PECS has grown significantly in recent years (Howlin, Gordon, Pasco, Wade, \& Charman, 2007), with more than 18 studies published between 2005 and 2009 alone (Bondy \& Frost, 2009). PECS has also been the topic of multiple reviews assessing its effectiveness (Flippin, Reszka, \& Watson, 2010; Hart \& Banda, 2010; Lancioni et al., 2007; Preston \& Carter, 2009; Sulzer-Azaroff, Hoffman, Horton, Bondy, \& Frost, 2009; Tien, 2008). The results of these reviews provide good evidence that PECS can be taught to individuals with ASD and DD. Lancioni et al. (2007), for example, provided one of the first reviews assessing the use of both PECS and VOCAs to enable individuals with DD to make requests. A total of 17 studies describing the 
use of PECS or equivalent systems, involving 173 participants (aged 3 to 40 years) were reviewed. These studies were published between 1992 and 2006. The results of the reviewed studies were largely promising, with only three of the 173 participants classified as failures. Still, Lancioni et al. suggested caution due to methodological concerns and the fact that PECS has so far mainly been used for teaching individuals to request highly preferred items, where one might therefore safely assume that a powerful motivational opteration is in effect.

In another relevant review, Flippin et al. (2010) summarised eight singlecase design studies, involving a total of 18 participants with ASD, and three group studies, including a total of 95 PECS participants and 65 other intervention/control participants. Participants receiving PECS intervention were aged 1-11 years. Flippin et al. concluded that PECS was effective and can be viewed as an empirically-validated communication intervention program. However, these researchers also noted concerns regarding the lack of ability to show whether PECS is associated with speech gains and the limited maintenance and generalisation data in most studies.

Systematic reviews of the literature specific to the use of graphic-mode and selection-based graphic symbol sets/systems in communication interventions for children with ASD and DD, but excluding PECS, have also been undertaken (Schlosser \& Wendt, 2008; Wendt, 2009). For example, similar to his review on MS, Wendt (2009) reviewed 15 studies on the implementation of graphic symbols, retrieved from his previously conducted systematic reviews and metaanalyses (Wendt, 2007). The studies were published between 1986 and 2005; all of which utilised single-case experimental designs and comprised a total of 43 participants (aged 4 to 40 years). Outcome variables of these studies included requesting and transitioning skills, identifying orthography, labelling under different instructional variables, and spontaneous picture card use. The results of this review suggested that such systems are promising, but there is insufficient evidence to point to the most effective instructional procedures.

\section{SGD Interventions}

SGDs are becoming more a widely studied AAC option for individuals with ASD/DD (Ogletree \& Harn, 2001; Schlosser, Sigafoos, \& Koul, 2009). SGDs are portable electronic devises that produce either digitised or synthesised speech 
output. SGDs typically display a variety of graphic symbols each of which may represent a word or phrase. When the word or phrase is activated, by touching or pressing a symbol, there is resulting voice-output (Mirenda, 2003). Being a selection-based communication mode, SGDs share many of the potential conceptual advantages and disadvantages of other aided AAC systems, such as requiring often complex conditional discriminations and scanning skills (Michael, 1985).

From a practical standpoint, a variety of SGDs can be selected and customised for intervention. SGDs can vary in design including graphic symbols used, permanence of the display (static or dynamic), number and size of graphic symbols on the display, shape, and size of the device (Drager et al., 2004; Drager, Light, \& Finke, 2009; Wilkinson \& Hennig, 2007). A defining feature of SGDs is the voice-output and consideration of the type of speech used (digitised versus synthesised) is also a potentially important variable to consider when designing interventions (Schlosser, 2003a; Schlosser \& Blischak, 2001; Schlosser, Blischak, \& Koul, 2003). Pre-recorded digitised speech is typically used in less complex AAC devices for beginning communicators and is presumed to be close to, or as intelligible as, natural (non-recorded) speech. Complex, hightech AAC devices utilise synthetic speech, which allow for a text-to-speech conversion, or the conversion of selected input to auditory output. Although this provides an unlimited amount of spontaneous speech, synthetic speech has been shown to be less intelligible than natural speech (Drager, Clark-Serpentine, Johnson, \& Roeser, 2006). Still the voice-output feature of SGDs might also make this a more readily understood mode of communication, compared to MS and PE (Rotholz et al., 1989).

Johnston and Feeley (2012) described two types of SGDs: dedicated and nondedicated. Dedicated SGDs are developed and used solely for communication purposes (e.g., Tech/Talk 6X8 TM , BigMack $^{\mathrm{TM}}$, GoTalk ${ }^{\mathrm{TM}}$, DynaVox®). Nondedicated SGDs include laptop/desktop computers, tablet computers, personal digital assistants, and cell phones. Special purpose software can be downloaded onto these nondedicated devices for use as AAC systems. Dedicated AAC devices have in the past been viewed as expensive, cumbersome, time-consuming to program and personalise, and often 
stigmatising to the user. Today there is an increase of interest in the use of nondedicated general-purpose hardware (e.g., iPods $₫$ and iPads $₫$ ), which is seemingly leading to a paradigm shift in aided AAC intervention. This shift is towards smaller, low cost, easy to obtain and transport, readily available, and socially acceptable devices (Shane et al., 2011). For example, a software application known as Proloquo2Go ${ }^{\text {TM }}$ (Sennott \& Bowker, 2009) runs on an Apple iPhone $\AA$, iPod Touch $\AA$, or iPad $\AA$ and can be programmed to serve as an AAC system. Mirenda (2009) noted several potential advantages of this system, including (a) the large set $(8,000)$ of colour graphic symbols, (b) the high quality of synthesised speech-output, (c) the relatively low cost, and (d) its small size and lightweight. In addition, many children with ASD appear to have an interest in technology and find computer activities to be reinforcing (Stromer, Kimball, Kinney, \& Taylor, 2006), making the use of such devices possibly well suited to individuals with $A S D$ and $D D$ who require alternative forms of communication. Despite these potential advantages, there are also some drawbacks to high-tech SGDs. Specifically, they are more prone to potential damage from being dropped or exposed to water, which can result in extended periods of time without the device while it is being repaired (Wilkinson \& Hennig, 2007). Passerino and Santarosa (2008) also explained that the mere use of technology is not enough to bring about changes in communicative interactions between people with ASD and DD. The strategies for teaching the individual to use the device are equally, if not more, important than the technology itself. For these reasons, new technologies, such as the use of $\mathrm{iPod}\left(\AA-\right.$ - (iPad $\left(\mathrm{B}-\right.$, iPhone $\left.{ }^{\circledR}-\right)$ based SGDs are beginning to be evaluated providing some of the first empirical data demonstrating the successful use of this new type of SGD (Kagohara et al., 2010; van der Meer, Kagohara, et al., 2011).

Although the literature is limited to only a couple of studies providing objective data on the use of high-tech nondedicated SGDs, there is other relevant research assessing the effectiveness of other SGD-based interventions (e.g., Brady, 2000; Franco et al., 2009; Sigafoos \& Drasgow, 2001) and also several literature reviews of these empirical studies (Schlosser, 2003a; Schlosser \& Blischak, 2001; Schlosser et al., 2003; Schlosser et al., 2009). Two recent reviews summarising the literature pertaining to the use of SGDs for children with 
ASD (van der Meer \& Rispoli, 2010) ${ }^{1}$ and for individuals with DD (Rispoli, Franco, van der Meer, Lang, \& Carmargo, 2010) ${ }^{2}$ provide evidence to support their use in communication interventions.

Specifically, van der Meer and Rispoli (2010) reviewed 23 studies involving 51 children (3 to 16 years of age) with ASD published between 1998 and 2009. Positive outcomes were reported for $86 \%$ of the studies and $78 \%$ of the studies were categorised as providing conclusive evidence. Rispoli et al. (2010) reviewed 35 studies involving 86 participants (1 to 42 years of age) with other DD. The studies were published between 1989 and 2009. Analysis of the outcomes of these studies showed that $86 \%$ of the studies resulted in positive outcomes, but only $54 \%$ of studies were categorised as capable of providing conclusive evidence. Still, the authors concluded that there was good empirical evidence to support the use of SGDs in communication interventions for individuals with ASD and DD.

Much like the literature on PECS, in both reviews, the majority of studies utilised single-case experimental designs and were predominantly focused on teaching functional (requesting) communication skills. Several studies did target some more socially-oriented communicative skills (e.g., Schepis, Reid, Behrmann, \& Sutton, 1998; Thunberg, Ahlsen, \& Sandberg, 2007; Thunberg, Ahlsén, \& Sandberg, 2009; Thunberg, Sandberg, \& Ahlsén, 2009). For example, in a study by Thunberg, Ahlsen et al. (2009), parents modelled the use of SGD to teach their children to use an SGD in conversational interactions during mealtime, asking and answering questions during story reading, and sharing experiences of their preschool day. Overall, the existing evidence suggested that SGDs are promising AAC options for individuals with ASD and DD. However, most studies focused on teaching requests for preferred objects.

\footnotetext{
${ }^{1} \mathrm{An}$ article based on this review has been published in Developmental Neurorehabilitation: van der Meer, L., \& Rispoli, M. (2010). Communication interventions involving speech-generating devices for children with autism: A review of the literature. Developmental Neurorehabilitation, 13, 294-306 (see Appendix B).

${ }^{2}$ An article based on this review has been published in Developmental Neurorehabilitation: Rispoli, M., Franco, J., van der Meer, L., Lang, R., \& Carmargo, S. (2010). The use of speech generating devices in communication interventions for individuals with developmental disabilities: A review of the literature. Developmental Neurorehabilitation, 13, 276-293 (see Appendix C).
} 


\section{Selecting AAC Systems}

With evidence to support the use of each of these three AAC systems (SGD, PE, and MS), Sigafoos, Drasgow, and Schlosser (2003) explained that an important decision for clinicians is the selection of a suitable AAC system for an individual. Traditionally, consideration was given to individual characteristics of the learner, his or her communicative partners, the environments in which communication occurred, and AAC system characteristics (Koul, Schlosser, \& Sancibrian, 2001; Reichle, 1991; Schuler \& Baldwin, 1981; Sigafoos \& lacono, 1993). Along these lines, guidelines were developed to assist clinicians in selecting an appropriate AAC option to meet the communicative needs and skills of individuals with ASD and DD. AAC assessment and intervention planning typically involved evaluation of the individual's current capabilities including receptive and expressive communication, symbolic understanding, literacy skills, motor skills, and sensory-perceptual limitations. Further, identification of communication needs was recommended to identify the vocabulary necessary to meet communicative obligations and opportunities, and which communicative intents should be taught. Cultural and family needs, as well as facilitator interaction strategies were emphasised to support the user's communication, and eliminate environmental barriers that might limit opportunities to communicate. Finally appraisal of system characteristics (see sections on MS Interventions, PE Interventions, and SGD Interventions, as well as Table 2.2 for a review) were recommended to select a suitable AAC system (Arthur-Kelly, Sigafoos, Green, Mathisen, \& Arthur-Kelly, 2009; Fossett \& Mirenda, 2007; Light, Roberts, Dimarco, \& Greiner, 1998; Reichle, 1991).

However, even with careful consideration of such factors there is no guarantee that the selected AAC system will be one that the individual is capable of learning and interested in using. Consequently some practitioners/researchers have recommended that individuals receive intervention with a number of different AAC systems (lacono, Mirenda, \& Beukelman, 1993; Reichle \& Ward, 1985; Sigafoos \& Drasgow, 2001). This use of multimodal communication (utilising both aided and unaided AAC) may also effectively capitalise on the unique strengths of each system, while minimising their respective disadvantages (Sigafoos \& Drasgow, 2001). 
Additionally, in order to implement an EBP approach for communication interventions, Sigafoos and colleagues (2003) outlined several other factors that should be considered. These involve collecting learner-generated performance data to assess the effects of the intervention on a regular basis and the importance of having an understanding of the basic principles and mechanisms that underlie empirically validated strategies. Nonetheless, decisions about AAC options are often based upon practical issues, rather than conceptual and empirical evidence (Sundberg \& Sundberg, 1990; Sundberg, 1993). This is perhaps because little research has been conducted to compare the differences between AAC systems (Koul et al., 2001; Sundberg \& Sundberg, 1990) despite the fact that recommending multiple systems from the outset may allow the opportunity to empirically determine which system is likely to be applied more successfully for any given individual (Shafer, 1993).

\section{Comparison of AAC Systems}

Schlosser (2003b) indicated that once an individual intervention approach has been demonstrated to be efficacious, it is important to know whether another approach might be more efficacious. Efficacy studies (utilising controlled experimental research methods), comparing acquisition of communication skills between various AAC systems, may be seen as a useful basis for selecting AAC modes. These analyses, however, have led to considerable debate within the literature regarding which of the various aided and unaided AAC options is best suited to individuals with ASD and DD (Mirenda, 2003; Schlosser \& Sigafoos, 2006).

A number of studies have compared topography-based (MS unaided) with selection-based (graphic-mode aided) AAC systems. Results from these studies (Sundberg \& Sundberg, 1990; Wraikat, Sundberg, \& Michael, 1991) provided empirical support for Michael's (1985) theoretical proposition that topographybased systems are acquired more efficiently and effectively than selection-based modes. In a review of these studies Potter and Brown (1997) noted that six of the seven studies reviewed reported more accurate responding in the topographybased condition. However, the majority of these studies consisted of adult participants with intellectual disabilities who did not have motor imitation deficits. In addition, the selection-based approaches utilised traditional picture pointing 
systems rather than PECS or use of SGDs, and intervention targeted tacting and intraverbal relations, rather than requesting (manding). In addition, the magnitude of the advantage for MS versus graphic-mode AAC tended to be rather small and hence probably not practically significant. For example, in a study by Bristow and Fristoe (1984), the average number of trials to reach criterion for the signing system was 3.95 , while it was 4.05 for the graphic-mode system. These differences were not statistically significant and most likely not clinically significant.

Research specific to teaching requesting (mands) to children with ASD and other DD, on the other hand, has indicated that there does not appear to be one AAC system that is appropriate for all beginning communicators (Sigafoos \& Drasgow, 2001; Sigafoos et al., 2003). In fact, Sigafoos and colleagues argued that the results from these comparison studies actually revealed no large nor consistent differences in terms how effectively and efficiently the various AAC systems can be taught to participants. In addition, studies comparing MS and PECS often yielded mixed results (Adkins \& Axelrod, 2001; Anderson, 2002; Gregory, DeLeon, \& Richman, 2009; Rotholz et al., 1989; Tincani, 2004). That is, some studies showed that PECS was learnt more successfully than MS (Adkins \& Axelrod, 2001; Rotholz et al., 1989), while other results indicated that children acquired both PECS and MS at an equally rapid pace (Gregory et al., 2009), or that acquisition varied between participants as a function of individual student characteristics (Tincani, 2004). Similarly, results of studies comparing PECS and SGDs for children with DD are also variable in terms of success of device acquisition (Beck, Stoner, Bock, \& Parton, 2008; Bock, Stoner, Beck, Hanley, \& Prochnow, 2005).

Few studies have compared SGDs and MS (lacono \& Duncum, 1995; lacono et al., 1993; Sigafoos \& Drasgow, 2001) and there do not appear to be any studies that have compared the acquisition of all three communication systems (SGD, PE, and MS). The lack of any major differences in the few available comparison studies specific to children with ASD and DD suggests that how quickly an individual acquires the use of an AAC option may not be the most critical variable to consider when selecting an AAC option for any given child. Instead, it is possible that the child's preference for using one AAC system over 
another is an important variable that should be considered when selecting an appropriate AAC system (Soto, Belfiore, Schlosser, \& Haynes, 1993). Giving individuals the opportunity to choose one AAC system over another may not only have positive effects on intervention outcomes, but enabling the individual to express his/her preference could also be viewed as one way of promoting selfdetermination in AAC interventions (Sigafoos, 2006). In line with these ideas Chapter 3 will further explain the potential advantages and theoretical implications of incorporating individual preferences into AAC interventions for children with ASD and DD. 


\section{CHAPTER 3 \\ RATIONALE FOR THE PRESENT RESEARCH}

\section{Self-Determination}

From a pragmatic perspective self-determination could be viewed as a way of enhancing interventions for children with ASD. That is, enabling children to self-determine aspects of the interventions they receive may be one way to enhance the success of the intervention. For example, if you know about a child's preferences you can incorporate those preferences into the intervention and thereby possibly increase the child's motivation to participate and learn (Reid \& Green, 2006). This view is consistent with an educational perspective of selfdetermination for individuals with disabilities (Stancliffe, 2001) described by Wehmeyer and colleagues (Wehmeyer, 1992; Wehmeyer, Palmer, Agran, Mithaug, \& Martin, 2000; Wehmeyer \& Shogren, 2008; Wehmeyer, Sans, Doll, \& Palmer, 1997; Wehmeyer, Shogren, Zager, Smith, \& Simpson, 2010). They suggest that acting as the causal agent in determining ones course of action is central to promoting self-determination. Causal agency infers that people make or cause things to happen in their lives (Wehmeyer \& Shogren, 2008). Therefore, self-determined behaviour refers to behaviour that is caused (i.e., determined) by oneself, as opposed to behaviour that is caused by someone or something else.

Self-determined people act volitionally (based on their own choices, preferences, and interests) instead of being made to act in certain ways by others or by circumstances (Wehmeyer et al., 2010).

Within Wehmeyer et al.'s (1997) model, self-determined behaviour consists of four essential characteristics: (a) actions are autonomous (according to individual preferences, interests, and/or abilities as well as independent and free from external influence or interference), (b) behaviours are self-regulated (actions as well as evaluation and revision of the actions, when necessary, are based on examination of the environment and ability to cope with the environment), (c) initiations and responses to events are psychologically empowered (involving control over circumstances that are important, skills necessary to achieve the desired outcomes, and, if the skills are used, identified outcomes will result), and (d) actions are self-realised (based on comprehensive 
and accurate knowledge of oneself, including strengths and weaknesses). Each of these four characteristics are necessary, to some degree, for behaviour to be self-determined (Wehmeyer et al., 1997). Such self-determined behaviour emerges through the development and acquisition of attitudes and abilities (referred to as component elements), such as choice-making, decision-making, self-advocacy, perceptions of efficacy and control, self-awareness, and selfknowledge (Wehmeyer, 1992; Wehmeyer \& Shogren, 2008; Wehmeyer et al., 2010).

From a behaviour-analytic perspective, self-determination is often conceptualised in terms of self-control (Wehmeyer, 1992). Skinner (1973), for example, argued that individuals show self-control when they control their own behaviour in precisely the same manner as they would control the behaviour of others (through the manipulation of variables of which the behaviour is a function). In this sense, self-control involves an individual engaging in some behaviour that in turn affects the variables that control that behaviour. For example, a person might set an alarm clock to control the time that they awake. Self-control in this view generally could be seen as involving the guiding, directing, and regulating of one's own behaviour, which might lead to desired consequences which then reinforce the self-control behaviour (Goldfried \& Merbaum, 1973). In this view a greater emphasis is placed on environmental influences of behaviour, rather than will or volition (Skinner, 1973). While this view would seem to be in conflict with views that see self-determination as involving the exercise of free will and volition (i.e., an inner determining agent), Skinner postulated that an analysis which draws on external variables makes the hypothesis of an inner determining agent unnecessary. One implication of such a view is that an analysis of the behaviour of self-control is possible and such an analysis should make it possible to teach the relevant components associated with self-determination.

\section{Choice Making and Personal Preferences}

Although individuals with disabilities have indicated that they would like to gain greater control over their own lives, research has established that these individuals are less self-determined than their non-disabled peers (Checkley, Nodge, Chantler, Reidy, \& Holmes, 2010; Stancliffe, 2001; Wehmeyer \& 
Shogren, 2008). They are frequently taught to depend on other people because they have been given limited opportunities to engage in self-determined behaviour, such as making choices and expressing personal preferences (Sigafoos, 1998; Wehmeyer, 1992; Wehmeyer et al., 2010). The reason for limited choice in the lives of people with disabilities may be explained by a lack of opportunities and perhaps also a lack of choice-making skills. The people who they interact with may also lack the skills needed to create opportunities for the person to make choices and express their preferences. These potential barriers to self-determination can be overcome to some extent by the systematic application of behavioural procedures to increase choice-making skills and create opportunities for choice-making and self-determination (Sigafoos, 1998).

An important direction for research is promoting self-determination in interventions for individuals with disabilities by providing individuals with choices regarding the therapies they receive (Arthur-Kelly et al., 2007; Odom et al., 2003; Sigafoos, 2006; Sigafoos et al., 2003; Stancliffe, 2001; Wehmeyer et al., 2000). Choice-making has been defined as the unforced selection of a preferred alternative from two or more options (Stancliffe, 2001). It can therefore be conceptualised as an operant response maintained by access to preferred items or events (Sigafoos, 1998). Choice-making often requires other people to provide the opportunity for choice-making and/or delivery of a chosen item. In these cases it involves a social-communicative interaction and thereby requires effective skills in communicating one's choices (Sigafoos, 1998). Individuals with ASD and DD, who have limited speech and language skills, may struggle to verbally communicate a preference for different options. Therefore, it would seem important to investigate whether there are other ways in which individuals might indicate which of several various options they would prefer.

Indeed, there has been considerable research on such approaches to assessing choice and preference for individuals with ASD/DD (e.g., Cannella, O'Reilly, \& Lancioni, 2005). From this research a number of methods have been proven valid, including (a) single stimulus, (b) paired stimulus, (c) multiple stimulus, (d) multiple stimulus without replacement, (e) single stimulus engagement, and (f) free operant (see Hagopian, Long, \& Rush, 2004). Of these different formats, the literature suggests that the paired stimulus, multiple 
stimulus without replacement, and free operant formats are the most commonly used (Hagopian et al., 2004). The multiple stimulus without replacement format (DeLeon \& Iwata, 1996), for example, involves presenting all items within a pool of potentially reinforcing items in an array and asking the participant to choose one (by pointing to, touching, or picking up the item). The participant is given the chosen item briefly before the trainer removes the item, and a subsequent trial is conducted. However, the chosen item is not placed back in the array in subsequent trials. This eliminates the chance of the participant choosing only one or a few items and allows the trainer to develop a rank order of items in terms of preference. The most preferred items can then be used within an intervention.

Utilising such choice-making formats and, by this means, enhancing communicative competence may allow individuals to gain some control and autonomy over their own lives thereby facilitating self-determination (Downing, 2009; Sigafoos, 1998; Stancliffe, 2001). Teaching new communication skills utilising $A A C$ and allowing individuals to choose which of the various $A A C$ options (SGD, PE, MS) they would prefer to use might therefore enhance the intervention outcomes and promote self-determination.

This prediction is consistent with research indicating that interventions incorporating self-determination lead to positive effects (Cobb, Lehmann, Newman-Gonchar, \& Morgen, 2009). For example, promotion of selfdetermination has been linked to improved quality of life and improved outcomes in areas such as academic achievement for individuals with and without disabilities (e.g., Carter, 2001; Fowler, Konrad, Walker, Test, \& Wood, 2007; Wehmeyer et al., 2000; Wehmeyer et al., 2010). Research has also indicated that when students are given the opportunity to make choices, reductions in problem behaviour and increases in adaptive behaviour will often occur (Cannella et al., 2005; Shogren, Faggella-Luby, Bae, \& Wehmeyer, 2004; Sigafoos, 1998). Specifically, it has been suggested that incorporation of preferred stimuli, which have positive and functional benefits for the individual, are more likely to result in the learning of, and continued use of a targeted skill (Sailor, Gee, Goetz, \& Graham, 1988). 


\section{Literature on Individual Preferences for AAC Options ${ }^{3}$}

Given these potentially positive effects of incorporating choice-making and specifically assessing an individual's preference for one communication option over another - on potential intervention outcomes, a review of relevant studies seems warranted. While this is a relatively new area of AAC research, with a small number of studies, a systematic review of these studies may facilitate EBP in at least two ways: (a) assist clinicians in their efforts to improve communication interventions for individuals with ASD and DD, and (b) identify areas in need of future research. Specifically, the systematic review that follows aimed to assess the methodology used in these studies in order to provide an initial evaluation of the viability for comparing acquisition and assessing AAC preferences in individuals with ASD and DD. Each study included in the review was summarised and discussed in terms of: (a) participants, (b) setting, (c) communication options assessed, (d) design, (e) communication skill(s) taught to the participant, (f) intervention procedures, including procedures for assessing preferences for different AAC options, (g) outcomes of the intervention and assessment of preference, $(\mathrm{h})$ follow-up and generalisation, and (i) reliability and treatment integrity.

\section{Method}

\section{Search Procedures}

Systematic searches were conducted in five electronic databases: Education Resources Information Center (ERIC), Medline, Linguistics and Language Behavior Abstracts (LLBA), Proquest, and PsycINFO. Publication year was not restricted, but the search was limited to English-language journal articles. The search covered all dates covered by these databases up to May 2010. For each database, the free-text terms augmentative and alternative communication and preference were inserted into the Keywords field. This initial search returned 48 articles. Abstracts of these 48 articles were independently examined by the author and an independent reliability data collector to determine

\footnotetext{
${ }^{3}$ An article based on this review has been published in Research in Developmental Disabilities: van der Meer, L., Sigafoos, J., O'Reilly, M., \& Lancioni, G. (2011). Assessing preferences for AAC options in communication interventions for individuals with developmental disabilities: A review of the literature. Research in Developmental Disabilities, 32, 1422-1431 (see Appendix D).
} 
if they met the criteria for inclusion in this review (see Inclusion and Exclusion Criteria).

Three additional search strategies were used to find other relevant studies that may have been missed by the electronic searches. First, the reference lists for the included studies were reviewed to identify additional articles for possible inclusion. Second, hand searches were completed for the journals that had published the included studies. Third, using an author search, the five databases were searched again for additional related work by authors of the studies that met the inclusion criteria. The reference list search yielded one additional article for possible inclusion in the present review, while the journal and author searches did not identify any further articles.

Finally, searches of the Journal of Applied Behavior Analysis (JABA), Journal of Developmental and Physical Disabilities (JDPD), and Research in Developmental Disabilities (RIDD) were undertaken because these journals have a history for including articles involving interventions that teach communication skills to individuals with DD. The keyword preference was entered into the search section of each of these journals' websites. Two hundred and thirty nine articles were identified in the JABA search, 140 articles in the JDPD search, and 284 articles in the RIDD search. The records returned from these electronic searches were independently reviewed by the author and reliability data collector to identify any further studies for inclusion in the review (see Inclusion and Exclusion Criteria).

\section{Inclusion and Exclusion Criteria}

To be included in this review, the article had to be a research study that included individuals with a diagnosis of a DD and involved a communication intervention that examined individual preferences between two or more AAC options. DD included individuals who were described as having autism, ASD, intellectual disability, or a related condition (e.g., developmental delay). AAC included both unaided (e.g., gestures or MS) and aided (e.g., PE or SGD) systems. Intervention was defined as implementing one or more therapeutic/teaching procedures for the purpose of trying to increase or improve the individual's communication skills through the use of AAC. Examples could include teaching an individual to use $A A C$ to make requests or answer questions. 
The research study had to include objective empirical data from which one could assess the success of the intervention and whether or not the individual showed a preference for using one AAC option over another. That is, the study had to include procedures for assessing whether participants showed a preference among two or more different $A A C$ options or provide evidence related to the extent to which the participants used one AAC option more than another. Studies that focused only on the description or assessment of communication skills were not included.

Of the original 48 studies identified during the initial systematic search, five met criteria for inclusion in this review. One of the 239 articles identified in the $J A B A$ search and one of the articles identified in the reference list search also met the inclusion criteria, resulting in a total of seven articles for inclusion in this review. There was $100 \%$ agreement between the author and reliability data collector as to which studies were to be included versus excluded.

\section{Data Extraction}

All studies meeting the inclusion criteria were coded by the first author in terms of: (a) participants (e.g., age, gender, number, and diagnosis), (b) setting (e.g., school, home or community setting), (c) communication options assessed (e.g., MS, PE, SGD), (d) design, (e) communication skill(s) taught to the participants, (f) intervention procedures (i.e., how communication skills were taught and how preference was assessed), (g) outcomes of the intervention and assessment of preference, (h) follow-up and generalisation, if any, and (i) reliability of data collection and treatment integrity. The results of the studies were analysed to determine the magnitude of preference for one communication option over another.

A summary of each of the seven included studies was written (see Table 3.1) with the accuracy of these summaries assessed by the reliability data collector using a checklist that included the initial table with summaries of the studies and a number of questions regarding various details of the study (i.e., Is this an accurate description of the purpose of the study?, Is this an accurate description of the participants?, Is this an accurate description of the communication options assessed?, Is this an accurate description of the design?, Is this an accurate description of the outcomes of the intervention?). There were 
Table 3.1. Studies Assessing Preference for Various Communication Options

\begin{tabular}{|c|c|c|c|c|c|c|}
\hline Study & Purpose & Participants & $\begin{array}{l}\text { Communication } \\
\text { Options Assessed }\end{array}$ & Design & Outcomes & $\begin{array}{l}\text { Magnitude of } \\
\text { preference } \\
\text { (selections/ } \\
\text { opportunities) }\end{array}$ \\
\hline $\begin{array}{l}\text { Canella- } \\
\text { Malone et } \\
\text { al. (2009) }\end{array}$ & $\begin{array}{l}\text { To compare } \\
\text { acquisition of } \\
\text { requesting for } \\
\text { specific items and } \\
\text { preference between } \\
2 \text { SGDs and a PE } \\
\text { system. To teach } \\
\text { functional use of the } \\
\text { preferred } \\
\text { communication } \\
\text { option. }\end{array}$ & $\begin{array}{l}2 \text { males, } 1 \text { with } \\
\text { mitochondrial } \\
\text { disorder and } \\
\text { significant } \\
\text { intellectual } \\
\text { disability ( } 13 \\
\text { years) and } 1 \text { with } \\
\text { neurological } \\
\text { disorder aetiology- } \\
\text { not-specified, } \\
\text { seizure disorder, } \\
\text { and significant } \\
\text { intellectual } \\
\text { disability (11 } \\
\text { years) }\end{array}$ & $\begin{array}{l}\text { Picture } \\
\text { communication } \\
\text { board; Mini- } \\
\text { messageMate } \\
\text { (digitised) and } \\
\text { Cyrano } \\
\text { Communicator } \\
\text { (synthesised) SGD. } \\
\text { Picture icons used } \\
\text { for all } 3 \text { AAC } \\
\text { devices were digital } \\
\text { colour photographs }\end{array}$ & $\begin{array}{l}\text { Multiple-probe- } \\
\text { across-AAC- } \\
\text { devices } \\
\text { design, with a } \\
\text { changing- } \\
\text { criterion } \\
\text { design during } \\
\text { functional } \\
\text { training }\end{array}$ & $\begin{array}{l}\text { Participant A only learnt to use } \\
\text { the PE system to request } \\
\text { preferred items; therefore did } \\
\text { not commence to the preference } \\
\text { assessment or functional } \\
\text { training. Participant B learnt to } \\
\text { use all three communication } \\
\text { options and demonstrated a } \\
\text { preference for the Cyrano } \\
\text { Communicator. Reached } \\
\text { criterion for functional training }\end{array}$ & $\begin{array}{l}\text { Cyrano } \\
\text { Communicator } \\
\text { selected } 65 / 78 \\
\text { opportunities } \\
(83 \%)\end{array}$ \\
\hline $\begin{array}{l}\text { Sigafoos, } \\
\text { Green, et } \\
\text { al. (2009) }\end{array}$ & $\begin{array}{l}\text { To compare the } \\
\text { effects of } \\
\text { acquisition (Study }\end{array}$ & $\begin{array}{l}1 \text { male with Down } \\
\text { syndrome and } \\
\text { autistic disorder }\end{array}$ & $\begin{array}{l}\text { PE system; } \\
\text { Tech/Talk 6X8 }{ }^{\mathrm{TM}} \\
\text { SGD (digitised). }\end{array}$ & $\begin{array}{l}\text { Alternating- } \\
\text { treatments } \\
\text { design }\end{array}$ & $\begin{array}{l}\text { Equally rapid acquisition of PE- } \\
\text { and SGD-based requesting } \\
\text { response. Slight preference for }\end{array}$ & PE 35/62 (56\%) \\
\hline
\end{tabular}


1) and preference

(Study 2) for a

SGD-vs. PE-based

requesting

response; and the

effects on social

interaction (Study 3)

Sigafoos

et al.

(2005)

To compare

acquisition of

requesting more

(i.e., "I want more")

snacks and

preference between

3 SGD

(Demonstration 1).

To compare

acquisition of

requesting specific

snack items and

preference between

the SGD chosen in

Demonstration 1

and a PE system

(Demonstration 2)

Son et al. To compare

(2006)

requesting of

specific items and

preference for a

SGD vs. PE system

(15 years)

Both AAC devices

used Picture

Communication

Symbols ${ }^{\mathrm{TM}}$ (Mayer-

Johnson Co, 1994)

2 males, 1 with BIGmack ${ }^{\mathrm{TM}}$ switch, autism (12 years)

and 1 with

intellectual

disability (16

years)

\section{2 females with}

autism ( 5 years, 5

months; 3 years, 8

months) and 1

male with PDD (3

years)
Talk $6 / 8^{\mathrm{TM}}$,

and Mini-

messageMate ${ }^{\mathrm{TM}}$

(Words+) SGD (all

digitised; Picture

communication

board. All AAC

devices used Picture

Communication

Symbols $^{\mathrm{TM}}$ (Mayer-

Johnson Co, 1994)

\section{PE system;}

Tech/Talk 6X8 TM

SGD (digitised).

Both AAC devices

used Picture

Communication

Symbols $^{\mathrm{TM}}$ (Mayer-

Johnson Co, 1994)
PE (56\%) over the SGD (44\%).

Distancing manipulation

resulted in significant reductions

in social withdrawal

Both participants learnt to use

all three SGD and the

communication board to make

requests. One participant

preferred the Mini-

messageMate ${ }^{\mathrm{TM}}$, while the other

preferred the Tech/Talk ${ }^{\top M}$. In

the second demonstration both

participants preferred the SGD

$(100 \%$ and $70 \%)$ over the PE-

based system

Alternating-

treatments

design

Little difference in acquisition of PE- and SGD-based requesting response. Two participants displayed preference for $\mathrm{PE}$ $(98 \%$ and $72 \%)$, while the third demonstrated a preference for the SGD (94\%). All children

used their preferred device with a high level of proficiency (86$100 \%)$
Demonstration 1 Jason =

Tech/Talk ${ }^{\mathrm{TM}}$

Ryan = Mini-

messageMate ${ }^{\mathrm{TM}}$

Demonstration 2

Jason =

Tech/Talk TM

22/31 (70\%)

Ryan $=$ Mini-

messageMate $^{\mathrm{TM}}$

6/6 opportunities

(100\%)

$\mathrm{Kim}=\mathrm{SGD}$

30/32 (94\%)

Lucy $=$ PE 86/88

(98\%)

Bruce $=\mathrm{PE}$

$52 / 72(72 \%)$ 
Soto et al. To compare

(1993) acquisition of requesting for specific items

(surrounding drawing and snacktime activities) and preference for a SGD vs. PE system in two settings.

Assess

generalisation and maintenance of requesting skills with the preferred AAC device

WinbornKemmerer et al. (2009)
To assess the effects of FCT to activate a SGD and touch a picture card to gain access to attention and tangible items in order to reduce challenging behaviour; and assess preference for one AAC option over the other
1 male with severe

to profound

intellectual

disability $(22$

years)

1 male with
pervasive
developmental
delays and seizure
disorder ( 7 years)
and 1 female with
intellectual
disability ( 20
years)

Picture

communication

board; Wolf ${ }^{\mathrm{TM}}$ SGD

(voice-output not

specified). Both AAC

devices used

Sigsymbols

(Cregan, 1984;

Cregan \& Llyod,

1990)

\section{Picture}

communication

system (picture of

therapist and an

item); BIGmack ${ }^{\mathrm{TM}}$

SGD (digitised

message: "Play,

please" or "Can I

have my_,please?").
Multiple-

baseline

design across

settings with

alternating

treatments

$(A=$ picture

card, $\mathrm{B}=$

SGD), and

multielement

design where

training

procedures

were randomly

drawn (i.e.

picture card or

SGD),

followed by the

alternate

condition and

another
The participant reached criterion in both settings for the SGD and

PE systems at similar rates.

Demonstrated preference for

the SGD $100 \%$ of the time, with

90 to $100 \%$ correct requesting.

Generalisation and maintenance demonstrated
SGD $100 \%$

Amount of

opportunities not specified
Problem behaviour and correct requesting increased for both participants as a function of the intervention. The choice analysis demonstrated that the male participant preferred the picture card, while the female preferred the SGD indicated by more responding

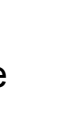

\section{Jack $=\mathrm{PE}$}

indicated by

more $(M=0.6)$

responses per

minute than

SGD $(M=0.2)$

Sally $=S G D$

indicated by

more $(M=0.6)$

responses per

minute than $\mathrm{PE}$

$(M=0.2)$ 
35 items on which there could be agreement or disagreement regarding data extraction and summarisation (i.e., 7 studies with 5 questions per study). Agreement was obtained on $100 \%$ of the items.

\section{Results}

A total of 10 interventions/experiments were reported in the seven included studies. Table 3.1 summarises the purpose, participants, communication options assessed, design, outcomes, and magnitude of preference for each of the seven included studies.

\section{Participants}

A total of 12 participants (8 males, 4 females) were included in the studies. Participants were reported as having a range of disabilities, including intellectual disability $(n=3,25 \%)$, autism $(n=3,25 \%)$, Down syndrome $(n=2,16.7 \%)$, PDD ( $n=2,16.7 \%)$, mitochondrial disorder $(n=1,8.3 \%)$, and neurological disorder aetiology-not-specified ( $n=1,8.3 \%$ ). Four (33.3\%) of these participants also had a combination of diagnoses (e.g., intellectual disability and autism).

Ages ranged from 2.8 to 22 years (mean $=10.9$ ). Sample sizes from the seven studies ranged from one to three participants. Only one study (Son, Sigafoos, O'Reilly, \& Lancioni, 2006) had three participants. Three (42.9\%) studies had one participant and the remaining three $(42.9 \%)$ studies had two participants.

\section{Settings}

Forty-three per cent $(n=3)$ of the interventions were undertaken in the participants' schools. Two (28.6\%) studies were undertaken in a clinical setting. One $(14.3 \%)$ study took place in the participant's home and the final study (Soto et al., 1993) was undertaken at the participant's group home and vocational centre, with generalisation probes conducted in a community setting (i.e., fast food restaurant).

\section{Communication Options Assessed}

Three different communication options were assessed in the 10 individual interventions reported: SGD, PE, and MS. A total of six different types of SGDs were used. Of the various SGDs used, the Tech/Talk 6X8 ${ }^{\mathrm{TM}}$ had the highest frequency $(n=3,50 \%)$, both the Mini-messageMate ${ }^{\mathrm{TM}}$ and $\mathrm{BIGmack}^{\mathrm{TM}}$ were 
used twice (33.3\%), and the Cyrano communicator, DynaVox® and Wolf ${ }^{\mathrm{TM}}$ were all used once. Four different types of symbols were used to represent messages on both the PE systems and SGD. Of these, the Picture Communication Symbols $^{\text {TM }}$ (Mayer-Johnson Co, 1994) had the highest frequency ( $n=3,75 \%$ ). digital photographs, DynaSims $®$, and Sigsymbols (Cregan, 1984; Cregan \& Llyod, 1990) were each used once. One study (Winborn-Kemmerer, Ringdahl, Wacker, \& Kitsukawa, 2009) did not specify what symbols were used to represent the messages used on the SGD- and PE-based systems. The one study (lacono \& Duncum, 1995) comparing MS and a SGD used signs from the Dictionary of Australasian Signs (Jeanes, Reynolds, \& Coleman, 1989).

The majority of studies ( $n=6,86 \%)$ compared how efficiently and effectively participants learnt to use SGD and PE systems. They also assessed which of these AAC systems participants preferred to use (see Intervention Procedures). Only one study (lacono \& Duncum, 1995) compared the use of MS with a SGD. Of the studies that included SGDs, two studies taught participants to use two (Cannella-Malone, DeBar, \& Sigafoos, 2009) or three (Sigafoos, O'Reilly, Ganz, Lancioni, \& Schlosser, 2005) different types of SGD to request access to preferred objects. Sigafoos et al. (2005) assessed which of these SGD participants preferred to use and continued intervention with the participants' preferred SGD, while simultaneously teaching the participant to use a PE system. They then assessed whether the participants preferred to use the SGD or the PE system.

\section{Design}

All of the studies included in the present review used some type of singlecase experimental design (Kennedy, 2005) to evaluate the effects of the intervention on communication using AAC devices. Specifically, all studies implemented baseline and intervention phases using some variation of a multiplebaseline and/or alternating-treatments design. Following intervention, six (86\%) of the studies implemented a choice assessment phase to determine which AAC option the participant preferred to use (see Intervention Procedures). The remaining study (lacono \& Duncum, 1995) assessed preference in terms of effectiveness of AAC device use. 


\section{Communication Skills Taught to the Participants}

The majority of studies ( $n=6,86 \%)$ taught participants some form of requesting as the primary communication skill. Fifty per cent $(n=3)$ of these six studies (Son et al., 2006; Soto et al., 1993; Winborn-Kemmerer et al., 2009) implemented interventions targeting requesting of specific preferred items (e.g., "I want chocolate, please"). One of these six studies (Sigafoos, Green, et al., 2009) taught the participants to make generalised requests for snacks (e.g., "I want a snack, please"). The remaining two studies (Cannella-Malone et al., 2009; Sigafoos et al., 2005) first taught participants to make a general request for preferred items and then taught the participant to request specific items (e.g., "I want a cookie." "I want a potato chip."). Two of these studies (Cannella-Malone et al., 2009; Sigafoos et al., 2005) also implemented an intervention to teach the participant how to turn on the SGD. One study (lacono \& Duncum, 1995) taught the sole participant expressive language skills (i.e., single word vocabulary, as well as two- and three-word phrases).

\section{Intervention Procedures}

Eighty-six per cent $(n=6)$ of the studies used systematic instructional procedures to teach communication skills. These procedures included (a) presenting an opportunity or discriminative stimulus, (b) prompting a communicative behaviour, (c) fading prompts, and (d) providing reinforcement for correct communicative behaviour. Within this broad class of procedures, some studies also identified more specific techniques as their core teaching mechanism, including (a) FCT (Winborn-Kemmerer et al., 2009), (b) graduated guidance (Sigafoos, Green, et al., 2009), and (c) least-to-most prompting (Sigafoos et al., 2005; Son et al., 2006; Soto et al., 1993).

Following intervention, these six studies implemented a structured choice making arrangement (e.g., Sigafoos, 1998) to identify a participant's preference for one AAC option over another. The general approach used by these studies involved making all communication options (i.e., both the SGD and PE system) available and then allowing the participant to choose one device. The remaining device/s were removed and a requesting opportunity was initiated with the chosen AAC option. When the participant consistently chose one option over 
another, it was considered to be his/her preferred mode of communication (see Outcomes of the Interventions and Assessment of Preference).

One study (lacono \& Duncum, 1995) implemented a child-directed approach. Intervention centred around activities that the participant had demonstrated an interest in, including pretend cooking and dressing up. Singleword vocabulary related to these activities was targeted for intervention. The treatment procedure involved providing models in response to the child's activities and initiations (e.g., hat when the participant put a hat on a doll) and specific reinforcement (e.g., that's right) if the participant imitated the model. This was followed by a two-word expansion. The trainer's models varied according to the treatment condition (MS or MS and SGD). A post-intervention phase was implemented with the most effective (in terms of word production) treatment modality, which was considered to be the participant's preferred AAC option.

\section{Outcomes of the Intervention and Assessment of Preference}

Outcomes for AAC device acquisition were classified and ranked into one of three outcome categories: (a) positive outcomes in which target communication skill(s) improved for all participants, (b) negative outcomes in which none of the participants improved in the target communication skill(s), and finally (c) mixed outcomes in which improvement was evident for some, but not all participants in the study, or in which some target skills improved and others did not.

Eighty-six per cent $(n=6)$ of studies reported positive outcomes in terms of participants learning to use the AAC devices for targeted communication skills. Only one study (Cannella-Malone et al., 2009) reported mixed outcomes, where one of the two participants did not reach criterion for AAC device acquisition, and therefore did not progress to the preference assessment phase of the study. No studies reported negative outcomes.

For the purpose of the present review a communication option was classified as highly preferred if it was selected on at least $70 \%$ of the provided opportunities, moderately preferred if it was selected on 55 to $70 \%$ of opportunities, and non-preferred if it was selected on less than $55 \%$ of the opportunities. For the 6 studies, involving 10 participants, that compared preference for SGD versus PE, $50 \%$ of the participants $(n=5)$ demonstrated a 
high preference for the SGD over PE. This included the study that first assessed preference between three SGDs and then assessed preference between the preferred SGD and a PE system (Sigafoos et al., 2005). Thirty per cent $(n=3)$ of the participants demonstrated a high preference for PE. One study (WinbornKemmerer et al., 2009) assessed preference in terms of amount of responses made with each AAC device per minute. For this study one participant exhibited a preference for the SGD, while the other participant preferred the PE system. The one study (lacono \& Duncum, 1995) that compared the combined use of MS and a SGD and MS alone found that the participant showed a preference for using the SGD, but the participant also showed an increased production of single-word vocabulary as well as two- and three-word phrases during the combined MS and SGD condition.

When comparing all three communication options (SGD, PE, and MS) across the seven studies included in this review, 67\% $(n=8)$ of the participants demonstrated some degree of preference ( $\geq 55 \%$ ) for using a SGD. Thirty-three per cent $(n=4)$ of the participants, in contrast, demonstrated some degree of preference ( $\geq 55 \%$ ) for PE.

\section{Follow-Up and Generalisation}

One study (Soto et al., 1993) undertook follow-up and generalisation probes. Only the preferred AAC option (SGD) was used during follow-up and generalisation. Communication skills maintained at a level of 66 to $100 \%$ correct requesting for four weeks. SGD use also generalised to different settings and different communication partners with 70 to $75 \%$ correct requesting.

\section{Reliability of Data Collection and Procedural Integrity}

All of the studies included checks on the reliability of data collection with respect to the dependent variables that is inter-observer agreement. Each of these studies reported average rates of inter-observer agreement above the generally accepted standard of $80 \%$ (Kennedy, 2005). Fewer studies $(57 \%, n=$ 4) reported procedural integrity data for the accurate implementation of intervention procedures. All of these studies reported high procedural integrity scores of over $80 \%$ correct implementation. One of these four studies (Sigafoos, Green, et al., 2009) did not collect procedural integrity data for one of the three demonstrations within their study. 


\section{Discussion}

This systematic search identified 10 interventions reported in seven studies published between 1993 and 2009. Five of these seven studies have been published since 2005, indicating an increase in assessing individuals' preferences between different modes of AAC and the potential impacts on intervention outcomes. In terms of quality of the evidence, all of the studies utilised appropriate single-case experimental designs with generally positive outcomes with respect to teaching requesting skills using each of the communication options assessed.

A key aspect of these studies was the attempt to assess preference for different AAC options. Structured choice-making arrangements (e.g., Sigafoos, 1998) were utilised to assess preferences for one mode of communication over another. Following the initial AAC intervention to teach requesting skills, the general approach used by the studies synthesised in this review was to give the individual an opportunity to choose which of the AAC options (SGD or PE) to use. When the individual consistently chose one option over another, it was considered to be his/her preferred mode of communication. Using this approach, it appeared that most participants did in fact appear to show some degree of preference for one AAC option over another. Because many AAC options exist and because it is difficult to predetermine whether any given option is viable, the preference assessment approach described in this review may be one way to enable individuals with DD to exert some control over their lives and participate in this important clinical decision.

However, results with respect to the certainty of evidence in terms of an individual's preference, or the 'magnitude' of preference, for one AAC option over another should be interpreted with caution. The literature pertaining to general choice-making and assessment of preference in individuals with DD (Sigafoos, 1998) defines preference in behavioural terms specifically as the frequency with which one selects an object or activity from an array of options. It is assumed that objects or activities selected more frequently are more preferred than objects or activities selected less frequently. The choice-making paradigm used in the studies included in this review could be seen as a type of preference assessment similar to those that have been used to identify stimuli for use as reinforcers 
(Cannella et al., 2005). Generally this preference assessment literature defines those alternatives that are selected or approached $80 \%$ of the time as preferred (Green et al., 2008; Pace, Ivancic, Edwards, Iwata, \& Page, 1985; Stafford, Alberto, Fredrick, Heflin, \& Heller, 2002). However, Son et al. (2006) defined an AAC option as preferred if it was selected on $70 \%$ or more of the total choice opportunities. Therefore, as mentioned, for the purpose of the present review, a communication option was also classified as highly preferred if it was selected on at least $70 \%$ of opportunities.

An important practical issue is how many choice opportunities are required to enable the demonstration of a preference for one mode of communication over another? In the studies analysed in the present review, participants were given between six and 88 choice opportunities. This is similar to other preference assessment research (Green et al., 2008; Pace et al., 1985; Stafford et al., 2002) where the range of choice opportunities has also varied greatly. With such disparity in the amount of opportunities individuals were given to choose one stimulus over another, reliably defining a percentage of selections for which an item can be considered preferred is difficult.

Similar to previous research (Beck et al., 2008; Bock et al., 2005), in the six studies that utilised a direct choice assessment (Cannella-Malone et al., 2009; Sigafoos, Green, et al., 2009; Sigafoos et al., 2005; Son et al., 2006; Soto et al., 1993, Winborn-Kemmerer et al., 2009) participants gained proficiency in using each AAC system relatively quickly and with comparable ease. Because participants had equal exposure to each device prior to assessing their preferences, they could be considered to be capable of making an informed choice when demonstrating preference for one mode of communication over another and that preference was generally not due to differences in performance between AAC options. However, it remains unclear why these individuals preferred one option over another. Son et al. (2006) and Sigafoos et al. (2005) suggested that there is perhaps something about the devices themselves (shape, colour, size, voice-output) or the way that they are used (pressing a switch on a SGD or handing over a card for PE) that makes them more or less appealing. Future research could investigate several such variables that might influence AAC preferences. 
It is widely documented in the choice assessment literature that preferences change over time (e.g., Stafford et al., 2002) and may need to be reassessed at regular intervals. This would seem necessary not only because giving an individual the opportunity to express preferences is important in its own right, but because it may also affect communication intervention outcomes. The studies examined in this review do not provide any results in terms of the effect preference may have on potential intervention progress and outcomes. In each of these studies, the child's preference for AAC options was assessed post-hoc; that is, the choice-making preference assessment was undertaken only after learning to use both options. This post-hoc assessment of preferences prevents an evaluation of the effects of preference on the child's progress during the communication intervention. It is unclear, however, whether these preferences can be identified during, and incorporated into, the beginning stages of intervention. Only one of the studies in this review assessed maintenance and generalisation of communication skills with the preferred AAC device (Soto et al., 1993). For the most part, it therefore remains unclear whether utilising preferred AAC options actually improves intervention outcomes in terms of promoting longterm maintenance of newly acquired communication skills. This limitation is important, as it is clear that individuals often abandon the use of AAC (Schlosser \& Lee, 2000). Findings indicating improved progress during intervention as well as better maintenance and generalisation of communication skills with the preferred device would provide further evidence for practitioners to allow individuals themselves to determine which communication option they would rather use.

Because this is an emerging field of AAC with a relatively small corpus of research, it is evident that the studies evaluated within this review are limited in several ways and that replications are warranted to address these limitations. Firstly, although the participant sample covered a good range of ages, diagnoses, and intervention contexts, the number is still limited with only 12 participants across the seven studies. It therefore remains unclear what percentage of individuals with DD will in fact show a preference for one mode of communication over another. 
Secondly, in six of the seven studies just two AAC options (SGD versus PE) have been compared. Only one study (lacono \& Duncum, 1995) compared MS and use of an SGD. Within the current sample the majority (67\%) of participants demonstrated some degree of preference ( $\geq 55 \%)$ for SGD. These results reflect recent reviews (Rispoli et al., 2010; van der Meer \& Rispoli, 2010) providing further evidence for the effectiveness of SGD-based communication interventions. However, preference for other AAC options, such as MS, and PE or comparison of SGD, PE, and MS, has not yet been investigated. It is therefore uncertain whether most children do in fact prefer SGDs over other communication options.

Third, in six of the seven studies the only dependent variable studied has been requesting behaviour. lacono and Duncuum (1995) taught expressive language skills and Winborn-Kemmerer et al. (2009) taught requesting in terms of FCT. Through FCT participants were taught appropriate communicative acts (to request a preferred item or to play) to replace their respective challenging behaviours. However, extension to more social and complex communicative interactions needs to be investigated. It remains unclear whether preference will influence how well the individual learns new and more advanced communication skills (e.g., commenting on the environment, initiating and maintaining conversations, and answering questions).

Finally, these studies have not undertaken a comprehensive assessment of the individual's skills and deficits to investigate which factors may influence AAC system acquisition and preference. It can be difficult for clinicians to determine the most appropriate mode of communication for an individual (Sigafoos \& Drasgow, 2001). Assessing learner characteristics, such as communicative and motor skills level, may predict not only which AAC modality the individual will be most effective at learning to use, but also which option he or she will prefer to use. For example, do children with more functional communication skills choose a more complex AAC device, such as a SGD? If clear patterns between learner characteristics, acquisition, and preference do emerge then selection of an appropriate AAC system for individuals with DD will be much easier to determine. Systematic replications to address these limitations 
would lead to further understanding on whether and how preferences will influence the effectiveness of AAC interventions.

It would also be important to determine whether preference for different AAC options might vary across situations and communicative partners. An SGD might, for example, be preferred for use in community settings, whereas MS might be preferred when communicating with peers who know MS. Such factors were not investigated in the present studies reviewed. Future research should therefore assess whether preferences for different AAC options generalise across situations and different communicative partners.

The present review has evaluated the feasibility and effects of incorporating aspects of self-determination into communication interventions for individuals with DD. Specifically it has assessed seven studies from an emerging field of AAC research incorporating a promising methodology to evaluate individuals' preferences for using one communication option over another. Findings suggest that while individuals are able to be taught to use alternative communication options (SGD, PE, and MS) for requesting, and often demonstrate a preference for one communication option over another, the evidence base supporting these findings are limited and the numerous variables that might influence $A A C$ preferences and use require future research. 


\section{CHAPTER 4 \\ OVERVIEW OF THE PRESENT RESEARCH}

\section{Purpose of the Research Project}

The research project presented in this thesis comprised three empirical studies which, together, sought to improve and expand on the systematic review of the literature included in Chapter 3. The overall prupose of these three empirical studies was to provide new knowledge on whether and how child preferences for particular AAC modes influence the success of the communication intervention. Generally, the studies were designed to evaluate the feasibility and effects of promoting self-determination in communication interventions for children with ASD and DD. Promoting self-determination predicts that better therapeutic success will occur when individuals are provided with choices and are enabled to express their preferences regarding the therapies they receive (Sigafoos, 2006). While previous research (see Chapter 3 ) suggested that individuals often demonstrated a preference for using one AAC option over another (e.g., SGD versus PE), it is unclear whether these preferences can be identified during, and incorporated into, the beginning stages of intervention. It is also unclear whether utilising preferred AAC modes actually improves intervention outcomes in terms of promoting acquisition and maintenance of communication skills. In accordance with these gaps in the knowledge base the present project aimed to answer the following research questions.

\section{Research Questions}

1. Is there a difference in how efficiently and effectively children with ASD and DD learn to use SGDs, PE, and MS when teaching an initial requesting skill?

2. Do children with $A S D$ and DD show idiosyncratic preferences for using SGDs versus PE versus MS?

3. If children with ASD and DD do show a preference for one AAC mode above the others, when does this preference first emerge; that is, does preference first emerge prior to or only after acquisition training? 
4. Does preference influence how efficiently and effectively children learn to use AAC?

5. Are preferences stable over time?

6. Does preference influence how well the child maintains initial communication skills?

\section{Hypothesis}

The project systematically addressed the above questions in the context of teaching new communication skills to children with ASD and DD utilising three AAC modes or options (i.e., SGD, PE, and MS). Preference assessments for these three AAC modes were designed to determine whether children made greater progress and maintenance of newly acquired AAC skills when their preferences for different AAC options were assessed and incorporated into the intervention process. Specifically, it was hypothesised that enabling children to choose which of the three communication modes to use (i.e., SGD versus PE versus MS) would enhance the success of the intervention in terms of acquisition and maintenance of the targeted communication skills. This hypothesis was based on research and theoretical perspectives suggesting that learning is enhanced by creating opportunities for children to make choices and thereby incorporating children's preferences into the intervention process (e.g., Sailor et al., 1988; as reviewed in Chapter 3).

\section{Methodology}

As outlined in the previous chapters, it would seem important to select interventions for individuals with ASD and DD based upon scientific evidence of their success, as well as the needs and circumstances of the individual learner. Similarly these interventions lend themselves to different research methods (Wehmeyer et al., 2010). Odom et al. (2003) suggested that leading interventions for individuals with ASD and DD utilise single-case research designs that integrate instructional strategies of prompting and reinforcement, assess individual characteristics and preferences, and utilise naturalistic environments, while simultaneously making use of advances in technology. The studies therefore made use of empirically validated $A B A$ approaches to teach $A A C$ skills, incorporated aspects of self-determination to assess child preferences for AAC modes, and the effects of intervention were implemented using single-case 
experimental designs. This approach is empirically defensible and methodologically sound for the purpose of assessing the efficacy intervention comparing relative performance with the three AAC options on an individual-byindividual basis (Schlosser, 2003c).

\section{Experimental Design}

The origins of single-case designs (Sidman, 1960) can be found in the work of Skinner (1953) and the emergence of ABA (Baer, Wolf, \& Risley, 1968), with research investigating basic behavioural processes, such as positive reinforcement. Single-case experimental designs are widely acknowledged as a valid approach for determining whether an intervention was in fact responsible for changes in the behaviour (e.g., learning) for individuals (Cozby, 2004). Generally when using a single-case experimental design for the purpose of evaluating the effects of an intervention, each participant's behaviour is first measured over time during a baseline control period during which the intervention is not applied. That is, the frequency of the target behaviour is measured before the intervention, which allows the researcher to identify changes in that behaviour in subsequent periods. The manipulation is then introduced during an intervention period while the participant's behaviour is observed. Evidence for the effectiveness of the manipulation is found when there is a change in behaviour from baseline to intervention. However, because there can be alternative explanations for the behaviour change, other than the intervention (e.g., history, practice, exposure), various types of single-case designs have been developed to reduce such threats to internal validity, including the multiple-baseline design (MBD) and alternatingtreatments design (ATD) that were utilised in the present research.

MBD was first introduced to the ABA literature by Baer and colleagues (1968). The MBD can provide convincing evidence that changes in the dependent variable (the target behaviour or task) are due to the introduction of the independent variable (the intervention procedure) because the intervention procedure is sequentially introduced to the participants. Presentation of the independent variable to the other participants is staggered across time. Any effects on the dependent variable replicated across participants when the intervention is introduced provide strong evidence to support the effectiveness of the instructional procedures. This design enhances the internal validity and 
controls for potential confounding variables, such as history, practice, and exposure, while systematic replications across participants and target behaviours increase the external validity (generalisability) of the findings (Kennedy, 2005).

Studies 1 and 2 of the present research used a variation of the MBD, namely the multiple-probe across participants design (Horner \& Baer, 1978). This type of design was developed to make use of the MBD more efficient for researchers by only intermittently collecting data during implementation of the experimental sequence (Kennedy, 2005). Study 3 of this research utilised a non-concurrent MBD (Watson \& Workman, 1981) because baselines were introduced at different points in time (Kennedy, 2005).

All three studies utilised an ATD design (Barlow \& Hayes, 1979; Barrett \& Sisson, 1987), which allows for comparison of the efficacy of two or more interventions (independent variables). After a baseline period two or more treatments are administered, alternating with each other, and the effects on behaviour (dependent variable) are observed. Merging two single-case design tactics (MBD and ATD) allows for more complex behavioural processes to be studied. Also, because experimental control can be demonstrated in multiple ways, demonstration of functional relation is often stronger than would be the case if only a single design was employed (Kennedy, 2005).

\section{Procedures}

The single-case designs utilised in this thesis involved the following sequential phases: baseline, intervention, preference assessments, postintervention, and follow-up. The baseline served to assess pre-intervention aptitude for the behaviours being measured. The independent variable was the combined operant instruction (see Instructional strategies) and AAC intervention. Because it has been recommended that communication interventions begin by teaching a simple requesting response if it does not develop naturally (Koul et al., 2001; Sigafoos \& Mirenda, 2002; see also Chapter 1), the studies involved teaching the participants to request (mand) preferred items using each $A A C$ mode (SGD, PE, and MS). The dependent variable was the frequency of correct responding (i.e., communicative requesting). Preference assessments were implemented throughout the studies (see Choice-making paradigm). Postintervention was undertaken to assess participant's preferences for the already 
learnt AAC modes. Follow-up was implemented to assess maintenance of the communication skills learnt. Follow-up probes are the most common design option for evaluating maintenance in AAC (Schlosser, 2003c). They are implemented at the end of the demonstration of a successful intervention by removing the independent variable (instructional strategies), waiting a specified time, and then measuring responding again.

Instructional strategies. In line with the theoretical basis of single-case research the studies presented in the present thesis utilised well-established instructional strategies based on the principles of ABA (Skinner, 1953). Specifically, the research utilised time-delay (Halle et al., 1979) and graduatedguidance (Azrin \& Armstrong, 1973) prompting methods, as well as reinforcement (outlined in Chapter 1). Time-delay, introduced by Halle et al. (1979), involves the trainer introducing a pause between the instruction and the delivery of a prompt. This provides the learner with an opportunity to independently respond to the instruction prior to the prompt. The constant time-delay procedure utilised in the present research involves introducing a time delay of a set length of time (e.g., 10 s) following the instruction. If the target behaviour occurs before the delay ends, the trainer provides reinforcement. If the target behaviour does not occur before the delay ends the trainer provides a prompt (Feeley \& Jones, 2012).

Graduated-guidance (Azrin \& Armstrong, 1973), explained by Duker et al. (2004), is a variation of the most-least prompting procedure, where the amount of physical guidance given by the trainer is gradually reduced as the learner's performance improves. Physical guidance involves using the least amount of hand-over-hand assistance needed to ensure the learner makes the target response with no possibility for error. As the learner begins to respond independently the trainer provides less and less physical guidance until the learner no longer requires physical guidance. If the learner stops responding the degree of physical guidance is increased. Graduated-guidance, therefore, requires the trainer to adjust the amount of prompting from moment to moment depending on the learner's developing independence in responding. In the present studies participants were provided with an opportunity to request a preferred item using one of the AAC modes; a $10 \mathrm{~s}$ time-delay was implemented 
between this onset of the opportunity (signalled by a verbal instruction by the trainer) and the use of graduated-guidance to prompt a correct request.

Choice-making paradigm. Participant's preferences for particular AAC modes were assessed throughout each phase of the study. Based on previous research (Cannella et al., 2005; Green et al., 2008; Pace et al., 1985; Piazza, Fisher, Hagopian, Bowman, \& Toole, 1996; Sigafoos, 1998; Stafford et al., 2002; Verschuur et al., 2011), a structured choice-making paradigm was implemented to evaluate children's preferences for the three AAC modes. Specifically, the choice-making paradigm (Sigafoos, 1998) involved systematic presentation of discriminative stimuli (the AAC modes) and allowed individuals to retain use of the one AAC option that was selected (see Table 4.1 for an outline of the procedures). When a participant consistently selected one AAC mode over the others ( $>$ than $55 \%$ of the time), it was considered to be his/her preferred mode of communication.

\section{Table 4.1. Structured Choice-Making Protocol}

1. Offer: Offer the person two or more items spaced some distance apart

2. Ask: While making an offer, ask the person which item he/she wants to use

3. Wait: Wait a reasonable amount of time (e.g., $10 \mathrm{~s})$ for the person to make a choice

4. Response: A choice occurs when the person makes any voluntary motion toward one of the items (e.g., points, reaches), maintains physical contact, or looks at one item for at least $3 \mathrm{~s}$

5. Reinforce: When a choice occurs, give the person the selected item (Sigafoos, 1998; Sigafoos, Roberts, Couzens, \& Kerr, 1993)

\section{Ethics}

Ethical approval was received from the Faculty of Education Human Ethics Committee at Victoria University of Wellington, New Zealand for the studies and manuscripts resulting from the research presented in the following three chapters (see Appendix A). Further, each study complied with the ethical standards of the American Psychological Association as well as the New Zealand Psychological Society. 


\section{CHAPTER 5}

\section{STUDY 1}

\section{Speech-Generating Devices versus Manual Signing in Children with Developmental Disabilities ${ }^{4}$}

\section{Aims and Hypothesis}

It appears that no research to date has implemented a choice-making arrangement to compare preferences for aided versus unaided AAC. Sigafoos and colleagues (2005) explained that it would be more complex to assess preference for using MS versus SGD, for example, because one cannot present MS in the same way that one can present a SGD. They suggested teaching the use of MS in the presence of a distinctive stimulus and the use of SGD in the presence of another distinctive stimulus. A choice condition could then be arranged by presenting both stimuli.

The present study aimed to employ such a procedure in order to assess preference for aided (SGD) versus unaided (MS) modes of AAC. Previous research highlighted the importance of utilising more sophisticated AAC devices that allow for individuals to expand on their communication capabilities (CannellaMalone et al., 2009), yet remain cost effective (Sigafoos et al., 2005). Therefore the present study used a new empirically validated (Kagohara et al., 2010; van der Meer, Kagohara, et al., 2011) software application known as Proloquo2Go ${ }^{\mathrm{TM}}$ (Sennott \& Bowker, 2009) run on an iPod®-based SGD, as well as signs from the Makaton sign language vocabulary (Makaton New Zealand/Aotearoa, 1998-99). In comparing preferences for this $\mathrm{iPod} \circledast$-based SGD versus MS, the present study also aimed to address some of the limitations addressed in Chapter 3 of the AAC device preference studies.

Specifically, the aim was to determine whether participants made greater progress, showed increased communication ability, and showed better maintenance of the AAC system for which they showed a preference. Based on previous research and theoretical perspectives (Chapter 3 ) it was hypothesised

\footnotetext{
${ }^{4}$ An article based on this study has been published in Research in Developmental Disabilities: van der Meer, L., Kagohara, D., Achmadi, D., O'Reilly, M., Lancioni, G., Sutherland, D., \& Sigafoos, J. (2012). Speech-generating devices versus manual signing for children with developmental disabilities. Research in Developmental Disabilities, 33, 1658-1669 (see Appendix E).
} 
that acquisition and maintenance of the two AAC options would be better for the AAC option that the participants also showed a preference for using.

\section{Method}

\section{Participants}

Four children were recruited from a special education unit in a public school. All four participants met the following criteria: (a) diagnosis of intellectual/developmental disability or ASD, (b) school aged children of less than 18 years of age, (c) very limited or no communication skills determined by an age level of 2.5 years or less in the expressive communication domain of the Vineland Adaptive Behavior Scales (Vineland-II; Sparrow, Cicchetti, \& Balla, 2005), (d) no auditory or visual impairments that would interfere with the use of AAC, and (e) sufficient motor skills to operate the AAC communication system, determined by an age level of 1.0 year or more on the fine motor skills domain of the Vineland-II.

David. David was a 10-year-old male diagnosed with ASD. On the communication sub-domain of the Vineland-II (Sparrow et al., 2005), David received age equivalencies of 3:11 (years:months) for receptive communication, 1:1 for expressive communication, and 4:3 for written communication. David did not have any spoken language. David received an age equivalency of 2:9 on the Vineland-Il for fine motor skills. At the time of the study he was using the Informative Pointing Method (Iversen, 2007), with assistance, as a form of communication. Teachers reported that David had limited experience with MS and PECS for expressive and receptive language prior to the present intervention. In addition to his severe communication impairment, David had limited social skills and engaged in frequent problem behaviour, including selfinjurious behaviour.

Tom. Tom was a 5.5-year-old boy diagnosed with Multi-System Developmental Disorder (MSDD) with autistic-like behaviours (e.g., impairment in reciprocal social communication, stereotyped and repetitive behaviours). His age equivalencies on the communication domain of the Vineland-II (Sparrow et al., 2005) were 0:11, 1:0, and 2:5 for receptive, expressive, and written communication. He received an age equivalency of 2.4 on the fine motor skills subdomain of the Vineland-II. Tom was able to use a few single words to make requests and comment on his environment, but he rarely spoke unless he was 
prompted by an adult. Prior to the present intervention Tom had been introduced to MS and PECS for receptive and expressive language, with limited success.

Zac. Zac was a 7-year-old male diagnosed with Down syndrome and ASD. He received age equivalencies of 1:3,2:1, and 4:10 on the receptive, expressive, and written communication domains of the Vineland-II (Sparrow et al., 2005). He received an age equivalent of 1:11 on the fine motor skills subdomain of the Vineland-II. Prior to intervention Zac used MS and PECS for expressive and receptive language. During the present intervention Zac received a GoTalk 2.0 ${ }^{\mathrm{TM}}$ SGD, which his teachers started to use with him. Although Zac attempted to speak frequently, his speech was largely unintelligible.

Eli. Eli was a 5.5-year-old male with Congenital Myotonic Dystrophy and autistic-like behaviours (e.g., impairment in reciprocal social communication, stereotyped and repetitive behaviours). His age equivalencies were 0:11, 0:8, and 2:5 on the receptive, expressive, and written communication domains of the Vineland-II (Sparrow et al., 2005). His age equivalency on the Vineland-II for fine motor skills was 1:8. Eli did not have any spoken language. He was introduced to MS for receptive and expressive language prior to the present intervention. $\mathrm{He}$ could use the signs for MORE and EAT. Aside from this, Eli made few communicative attempts. He engaged in frequent stereotypic (self-stimulatory) behaviour and was said to often fixate on certain objects, such as computers.

\section{Setting and Intervention Context}

All four participants attended the same classroom at a special education unit for children with disabilities that was part of a public primary school. Fourteen additional children were educated in this classroom by two teachers and six teaching assistants. The procedures related to this study were conducted at a table in a small room that linked to the main classroom during a morning snack/leisure activity. The procedures were implemented in a one-to-one context consisting of the trainer (author) and one participant at a time. No one else was present in the room, other than one or two reliability and procedural integrity observers (who sat in the corner) on some occasions.

\section{Preferred Stimuli}

Snacks and/or toys that the participants seemed to prefer, and which would be appropriate for the participants to request during the snack/leisure 
activity, were identified by a systematic two-stage stimulus assessment (Green et al., 2008). Stage 1 involved an indirect assessment in which both teachers were asked to list snack foods, sensory stimuli, and toys that the participants appeared to enjoy and would be appropriate for the classroom. Three to six of the most preferred food and play stimuli were then selected for a direct stimulus assessment. The direct method for assessing preferences involved the simultaneous presentation of multiple items, without replacement (DeLeon \& Iwata, 1996; Duker et al., 2004). Each participant was presented with an array of preferred items (random placement) and allowed to select one. Items were not replaced once they had been selected, thereby eliminating the chance of the participant choosing only one or a few items, as well as allowing the trainer to develop a rank order of items in terms of preference. Play and food items were assessed separately. The top two to four food and play items were identified by calculating the percentage of times that the stimulus was selected, out of six trials (across four sessions) in which each stimuli were offered.

Preferred stimuli for David included a venting ball (46\%), dental floss $(43 \%)$, and water play (represented by a container filled with water and plastic toys; $29 \%$ ) for toy items; fruit-leather (66\%), M\&Ms (40\%), and chocolate and gherkins (33\% each) for snack items. Preferred stimuli for Tom included toy cars (100\%), bubbles (42\%), and building blocks (35\%) and a lollipop (86\%), muesli bar (42\%), and crackers (22\%). Preferred stimuli for Zac included an alphabet musical toy (55\%), a Hi5 CD (40\%), and playing on the computer (30\%) and juice (50\%) and chips (20\%). Preferred items for Eli were bubbles (67\%), paper (43\%), and an alphabet musical toy (38\%) and peppermints (75\%), chips (46\%), and juice (31\%). All participants, excluding Zac (his parents indicated a preference for teaching play items, not snacks), received baseline with both the preferred snack and play options. David initially received training to request both snack and play items. However, due to time constraints it was decided that David would be taught to request snacks only (at Session 8 of Figure 5.4), while Tom, Zac, and Eli were taught to request toys only. Snacks for David and toys for the other children were identified as the more preferred options in the direct stimulus assessment. 
SGD

Participants were taught to request their preferred toys or snacks using an Apple iPod Touch ${ }^{\circledR}$ with Proloquo2Go ${ }^{T M}$ software (Sennott \& Bowker, 2009). The $\mathrm{iPod} \circledast$ was placed inside an iMainGo2 ${ }^{\circledR}$ speaker case to increase sound

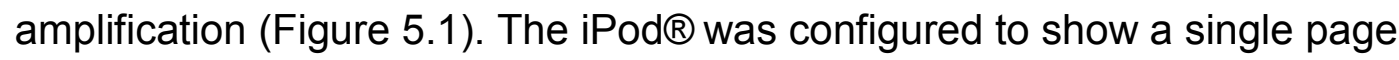
containing three $(2.5 \times 2.5 \mathrm{~cm})$ graphic symbols, representing requests for SNACKS, PLAY, and SOCIAL INTERACTION. Touching each symbol activated corresponding synthetic speech-output (i.e., "I want a snack please.", "I want to play.", and "How are you?").

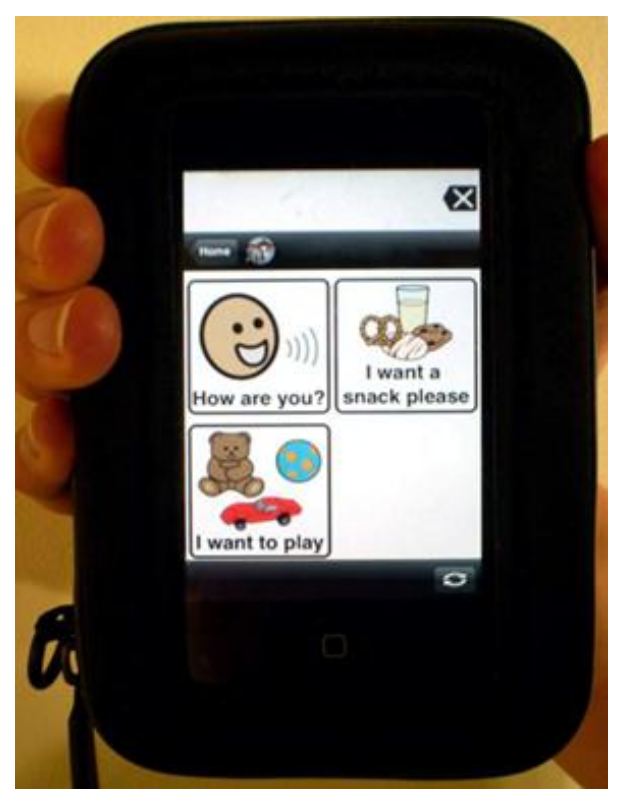

Figure 5.1. Photograph of the iPod Touch ${ }^{\circledR}$ inside the iMainGo ${ }^{\circledR} 2$ Speaker Case with the Visual Display of the Graphic Symbols for SOCIAL INTERACTION, SNACKS, and PLAY for Study 1.

\section{MS}

Participants were also taught to request preferred stimuli using manual signs from the Makaton sign sanguage system (Makaton New Zealand/Aotearoa, 1998-99). Participants were taught the signs for SNACK or PLAY. The MS option was represented by a laminated photo $(16 \mathrm{~cm} \times 11 \mathrm{~cm})$ of the trainer making the hand formations for the signs for SNACK and PLAY (Figure 5.2). 


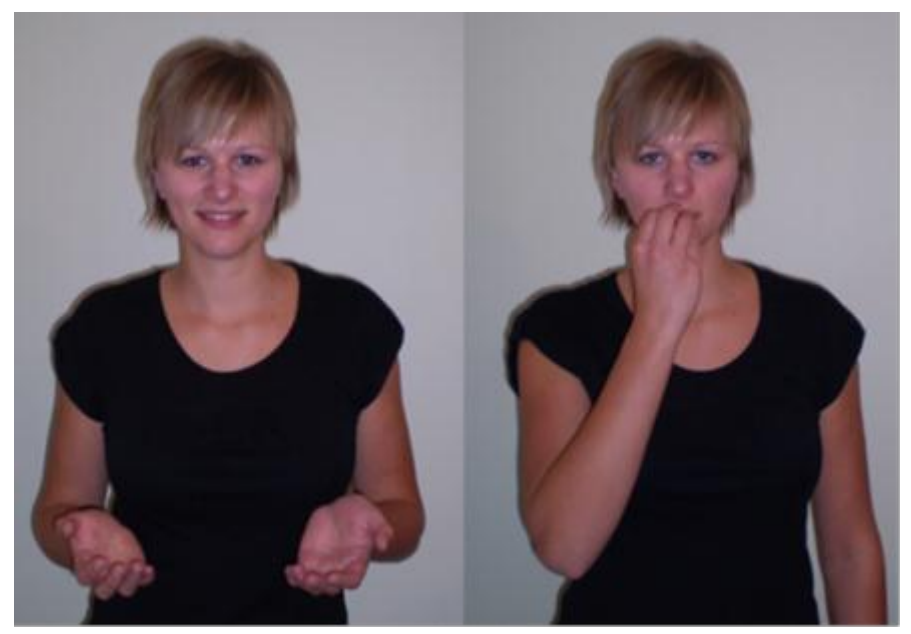

Figure 5.2. Photograph of the Trainer Making the Signs for PLAY and EAT to Represent the MS Communication Option for Study 1.

\section{Response Definitions and Measurement}

For SGD use, correct responding was defined as independently (without a gestural or verbal prompt) touching the symbol on the screen of the SGD to activate the corresponding speech output in exchange for a desired item from the trainer. MS was defined as independent (without a gestural or verbal prompt) hand gestures to produce correct signs in exchange for a desired item from the trainer. Independent approximations of the sign (e.g., rotating hands once or several times for play, instead of exactly twice) were counted as correct. The frequency of correct responding was calculated for each session consisting of 10 offers of snack or play items. The target response for David was touching the SNACK symbol on the SGD to activate the message "I want a snack please.". The PLAY and SOCIAL INTERACTION symbols were distracters. David's target response for MS was to produce the sign for SNACK. The PLAY sign (on the laminated card) was a distractor. The target response for Tom, Zac, and Eli was touching the PLAY symbol on the SGD to activate the message "I want to play.". The SNACK and SOCIAL INTERACTION symbols were distracters. The MS target response for Tom, Zac, and Eli was to produce the sign for PLAY. The SNACK sign (on the laminated card) was a distractor.

\section{Experimental Design}

The study included the following phases arranged in a multiple-probe across participants design (Horner \& Baer, 1978): Baseline, intervention, 
preference assessment (throughout intervention, post-intervention, and followup), post-intervention, and follow-up. An alternating-treatments design (Barlow \& Hayes, 1979; Barret \& Sisson, 1987) was also embedded within the multipleprobe to compare performance of the SGD and MS options.

\section{Session Schedule}

Two to four requesting sessions were conducted three to four days per week. Each session lasted about $5 \mathrm{~min}$ and consisted of 10 discrete trials. The AAC option available during a session (i.e., SGD versus MS) was alternated across sessions. Whether training commenced with SGD or MS was random depending on how many sessions were implemented on the previous day of training. For all sessions, the participant and trainer were seated next to each other at a table with one or two additional observers to establish reliability and procedural integrity.

\section{Procedures}

Because participants were at the beginning stages of intervention they were taught to request only one type of highly preferred item. However, to ensure some level of symbol discrimination, the distractor symbols/signs were included in the SGD and MS options. If participants requested a snack when they were undertaking training to request a toy or vice versa, the trainer explained We are learning to request toys/snacks at the moment, you can request a toy/snack another time. Activating the SOCIAL INTERACTION symbol on the SGD resulted in a brief spoken reply from the trainer (e.g., I'm good thanks, how about you?). It was considered more natural to provide participants with some feedback when they activated these (non-target) symbols/produced these (non-target) signs, but not to reinforce these with preferred tangibles. Similarly, producing MS to request items during SGD sessions was ignored in order to bring the use of each device under stimulus control.

Baseline. During baseline, a tray containing three different snack (toy) items was placed on the table in view, but out of the participants' reach. The SGD was placed on one side of the table in an upright orientation. A photo of the trainer signing was used to present the MS option. The photo was placed on the other side of the table, also in an upright orientation. Each session involved one block of five discrete trials for snacks and one block of five discrete trials for toys, 
with the order of blocks counterbalanced across sessions. The session began with the trainer telling the participant: Here is a tray of snacks (toys), let me know if you want something. After $10 \mathrm{~s}$, the trainer moved the tray within reach and allowed the participant to take one item. This was repeated across 5 discrete trials each for snacks and toys. This 10 -s fixed time schedule of reinforcement was provided to ensure continued motivation to participate in sessions. When offering snacks, participants were allowed to select one item from the tray, which was then replenished before the next offer. When offering the tray of toys, participants were allowed to select one toy and play with it for approximately $30 \mathrm{~s}$ before it was returned to the tray. SGD and MS responses were recorded, but had no programmed consequences.

Intervention. This phase was conducted in a discrete trial format until participants reached criterion (i.e., 80\% correct requesting across 3 consecutive sessions for each AAC option). Either the SGD or the MS option was placed on the table (see Session Schedule) in reach of the child in accordance with the alternating treatments design. Each trial consisted of the trainer pointing to a tray of toys (snacks) and saying: Here's a tray of toys (snacks). Let me know if you want something. Training involved a 10-s time delay between the verbal cue (i.e., Let me know if you want something) and the use of graduated guidance to prompt a correct request. Graduated guidance involved use of the least amount of physical guidance necessary to ensure the child made a correct request, while simultaneously explaining the required response (e.g., Press PLAY to ask to play with a toy. or Move your hand to your mouth to make the sign for SNACK.). Immediately after the speech output occurred, or the correct sign was made, the trainer moved the tray containing the toys (or snacks) within reach of the participant. The participant was allowed to select one item from the tray and consume the chosen snack or play with the chosen toy for about $30 \mathrm{~s}$. After this, the next trial was initiated.

Procedural Modifications. Because Tom made little progress with acquisition of MS during the initial intervention sessions, his teaching procedures were modified to include 10-s time delay followed by a least-most prompting procedure. This involved first tapping Tom's elbow, then lifting his forearm, followed by a full physical prompt if he did not make the sign correctly. However, 
because progress was not observed with this procedure, a 30-s time delay followed by graduated guidance, as well as differential reinforcement (where Tom was only given the opportunity to play if he independently used the sign to request to play), was introduced. Prompted trials were not reinforced.

Another participant ( $\mathrm{Zac}$ ) did initiate correct requests during the initial intervention sessions, but he appeared to have trouble physically performing the task independently for both MS and the SGD. It was therefore decided to implement massed training trials before each SGD and each MS session. This involved 10 consecutive graduated guidance trials with no time delay and no reinforcement per session to get Zac to perform the task as independently as possible.

AAC Preference Assessment. These assessments were undertaken to determine if participants would show a preference for using one of the two AAC options. These assessments occurred after every eighth session (i.e., after four MS and four SGD sessions) on average. This number varied slightly because these assessments had to occur before the first session for the day to prevent sequence effects (selecting the AAC option that was taught last; Sigafoos et al., 2005), as well as to ensure that at least two such preference assessments were undertaken during intervention for each participant.

During a preference assessment, the trainer presented the MS option on one side of the table and the SGD option on the other side of the table (alternated across sessions to control for choice being made dependent on location of the AAC option). The trainer asked the participant: Which communication option would you like to use? Sign language on this side (while pointing), or the SGD on this side (while pointing)? The trainer initiated one requesting opportunity with the chosen $\mathrm{AAC}$ option before reverting back to initiating requesting opportunities with the AAC device that was being used for that session. Choice for an AAC option was defined as physically pointing to, touching, or picking up the selected communication option. If the child did not choose an option within $10 \mathrm{~s}$, the device preference assessment was terminated and training continued with the AAC option that was scheduled for use in that session.

Post-intervention. Once the participant reached criterion with both the MS and SGD options, post-intervention preference assessments were 
introduced. These were identical to previously described device preference assessments, except that once an AAC option had been chosen, the participant continued to request preferred items using the chosen communication method for the entire session. A minimum of four of these post-intervention preference assessments were undertaken for each participant.

Follow-up. David received three sets of follow-up sessions, at 2 weeks, 1 month, and 6 months. The remaining participants received two sets of follow-up sessions at 1 month and 6 months. David received four follow-up sessions two weeks after his last post-intervention session. During the two-week interval, David was on midwinter break, did not attend school, and did not use either communication option. All participants received four follow-up sessions one month after their final intervention or post-intervention session. Participants did not use either communication option for requesting preferred items during the one month interval before these follow-up sessions. Six months later, another four follow-up sessions were conducted for all participants. During the interval between the 1 and 6 month follow-up sessions, the participants had access to iPods $₫$ and iPads $₫$ for educational and leisure activities, but did not use the iPod®-based SGD or MS communication options for requesting preferred items. Procedures for follow-up were identical to the intervention phase, except no teaching occurred and reinforcement was contingent upon a correct request. A device preference assessment was implemented before each session.

\section{Inter-Observer Agreement}

The trainer collected data on the frequency of correct requesting, the level of prompting required during intervention for each trial, as well as which communication mode was selected during device preference assessments. To assess the reliability of the trainer's data collection, an independent observer also collected data on the frequency of requesting, level of prompting, and communication mode chosen. For each session, percentages of agreement between the independent observer and the trainer were calculated using the formula: Agreements/(Agreements + Disagreements) $\times 100$. Reliability of data collection was obtained by the independent observer on $43 \%$ of all sessions and ranged from 90 to $100 \%$ with an overall mean of $99.6 \%$. 


\section{Procedural Integrity}

To assess procedural integrity, the independent observer used a checklist of the procedures and recorded whether the trainer had correctly implemented each procedural step in its proper sequence. The independent observer assessed procedural integrity during $43 \%$ of all sessions, which ranged from 98 to $100 \%$ with an overall mean of $99.9 \%$. A second independent observer collected inter-observer agreement data on $21 \%$ of these integrity checks with $100 \%$ agreement.

\section{Results}

Figure 5.3 provides a summary of the results from the AAC preference assessments across each phase of the study. Figure 5.4 shows the percentage of correct requests during each session for both AAC modes across participants. During baseline, participants never used MS to request snacks or toys. They did occasionally touch the MS card, pick it up, or play with it for a few seconds. Eli used the SGD to request toys or snacks on $20 \%$ of the baseline trials. Many of these requests appeared to be made by chance as Eli repeatedly touched the icons on the SGD representing items (snack or toy) that were not being offered on that trial. Although none of the other participants activated the SGD, they did occasionally touch it, pick it up, or play with it for a few seconds. Also, during baseline, all participants often reached for the tray of snacks or toys before it was offered to them (these responses were blocked). Zac attempted to verbally request certain items from the tray, but this speech was mostly unintelligible. When the tray of snacks or toys was offered, David and Tom selected and consumed or played with items $98 \%$ of the time, Zac did so $100 \%$ of the time, and Eli did so $94 \%$ of the time.

In order to calculate effect sizes Nonoverlap of All Pairs (NAP) was used. NAP is "the percent of non-overlapping data between baseline and treatment phases" (Parker \& Vannest, 2009, p. 359), or in other words "the probability that a score drawn at random from a treatment phase will exceed (overlap) that of a score drawn from a baseline phase" (p. 359). For example, that the frequency of correct requesting in intervention is higher than in baseline. It is used to determine the usefulness of the intervention procedures on teaching target behaviours to the participants. Table 5.1 shows the resulting percentages of 
NAP. Effect sizes for each participant for treatment effects from baseline to intervention across both communication options (SGD and MS) were calculated. Parker and Vannest (2009) explained that "large" effect sizes are generally equal

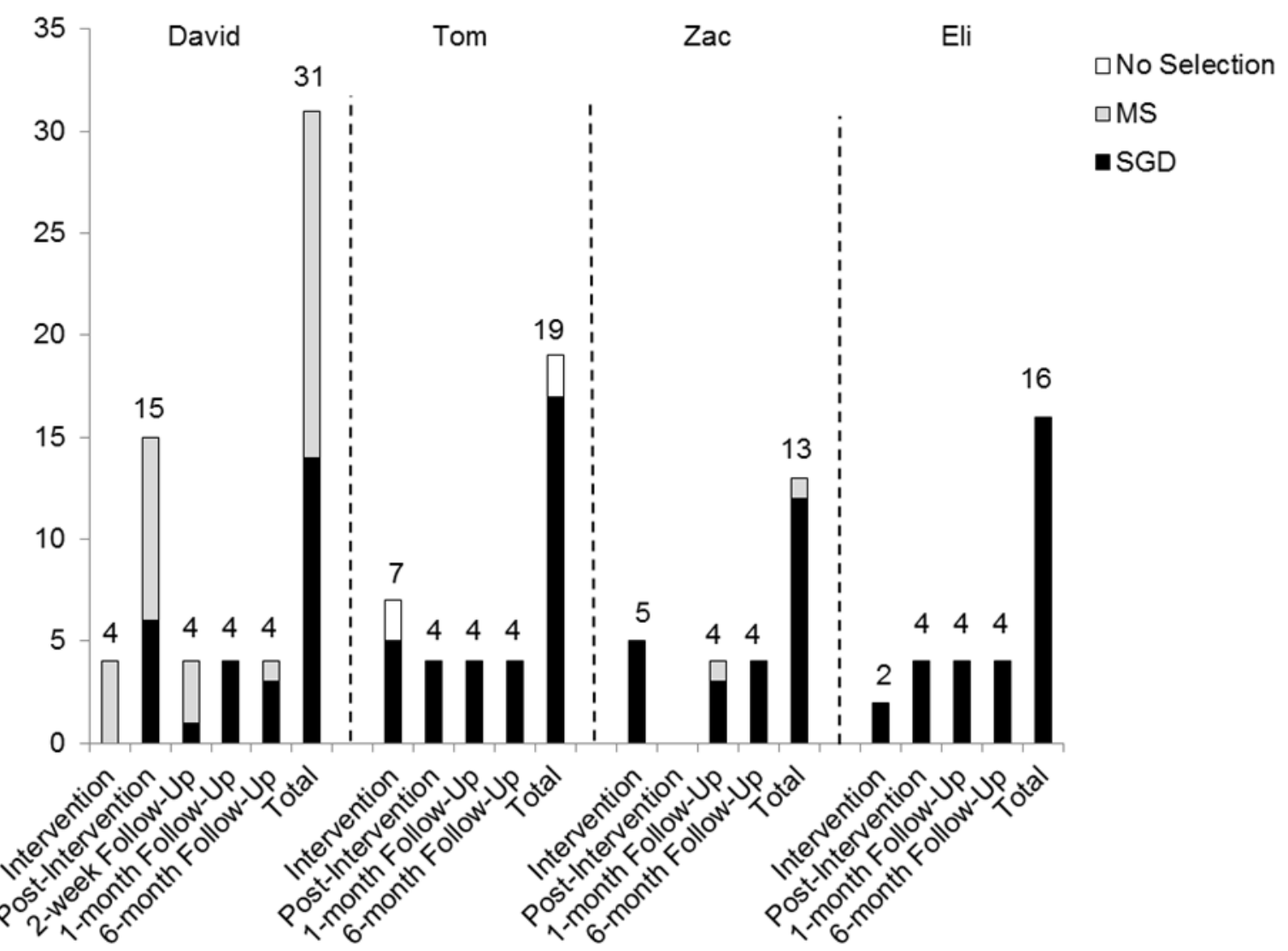

Figure 5.3. Results from the AAC Preference Assessments Depicting the Number of Times each Communication Option (SGD and MS) was Chosen across Phases and Participants.

to or greater than $80 \%$, but warned that these guidelines given by Cohen (1988) are from large $N$ social science research and should not be readily applied to other contexts. Nevertheless, "large" effect sizes were found for three out of the four participants (Tom and Eli 100\%, Zac 82\%) for intervention effects for SGD. However, only two out of the four participants (David 92\%, Eli 100\%) received such high intervention effects for MS. It appeared that greater effects across participants were achieved for the SGD communication option $(M=89.5 \%)$ relative to MS $(M=60.5 \%)$. The same three of four participants with high effect sizes for SGD also demonstrated a preference for using the SGD. Descriptions of each participant's acquisition and preference are provided below 


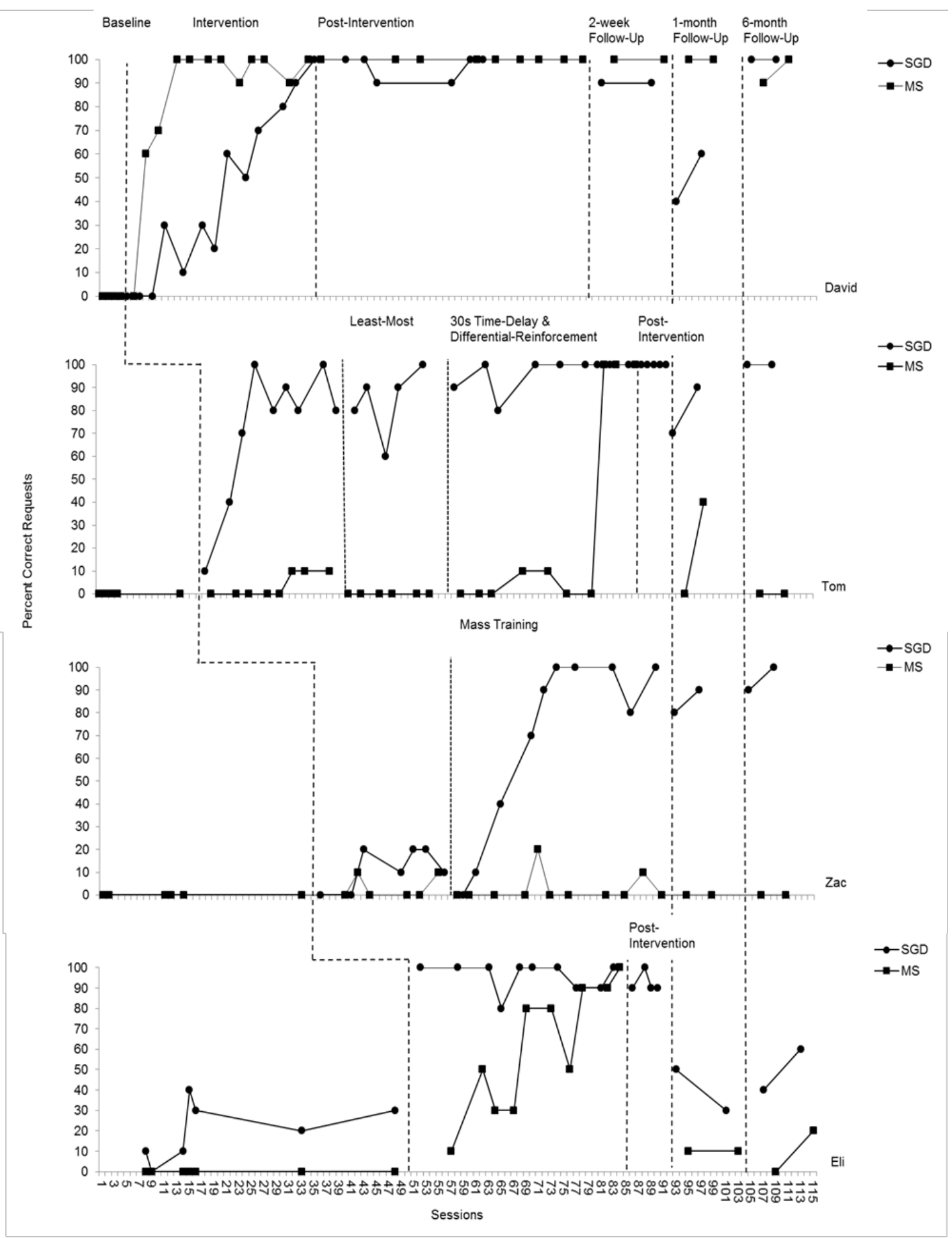

Figure 5.4. Percentage of Correct Requests with SGD and MS across Sessions for each Participant. 
Table 5.1. NAP Effect Sizes from Baseline to Intervention for the SGD and MS Conditions.

\begin{tabular}{lll}
\hline Child & SGD & MS \\
\hline David & $76 \%$ & $92 \%$ \\
Tom & $100 \%$ & $26 \%$ \\
Zac & $82 \%$ & $24 \%$ \\
Eli & $100 \%$ & $100 \%$ \\
\hline
\end{tabular}

NAP is rescaled to a $0-100 \%$ scale

\section{David}

David achieved criterion of requests for snacks using MS on his $6^{\text {th }} \mathrm{MS}$ session (Session 18 of Figure 5.4). He achieved criterion using the SGD on his $13^{\text {th }}$ SGD session (Session 35 of Figure 5.4). During intervention, David received four device preference assessments in which he chose MS on each occasion. A second device preference assessment was undertaken for David three sessions after the first to ensure the accuracy of the initial device preference assessment because a correct response had to be prompted. Because the results were consistent, this was not repeated for other participants where a response had to be prompted. During the post-intervention phase David chose MS on $60 \%$ of sessions and used it with $100 \%$ proficiency during subsequent requesting opportunities. He chose the SGD $40 \%$ of the time and correctly used it 90 to $100 \%$ of the time.

During his first set of follow-up sessions, David maintained requesting for snacks using both the SGD and MS at a high level. He chose the MS option on three out of four ( $75 \%$ ) of preference assessment opportunities and used it with $100 \%$ proficiency. While MS maintained at $100 \%$ correct requesting during his second set of follow-up sessions (one month after the initial follow-up), SGD use dropped to 40 to $60 \%$ correct requesting. Interestingly, David chose the SGD on $100 \%$ of the device preference assessments, but on three out of these four occasions he did not make a correct request using the SGD. At the six month follow-up, MS use maintained at 90 to $100 \%$ correct and David demonstrated increased SGD performance with 100\% correct requesting. Again, David showed a preference for the SGD, selecting it on $75 \%$ (3 out of 4) of opportunities. Overall David received a total of 31 opportunities to choose between the SGD and MS (Figure 5.3) and chose MS (55\%) on a slightly higher percentage of occasions. 


\section{Tom}

Tom achieved criterion of requests for toys using the SGD on his $6^{\text {th }}$ SGD session (Session 31 of Figure 5.4), but he made little progress with MS. On his $9^{\text {th }}$ MS training session (Session 41 of Figure 5.4), the least-most prompting procedure with a 10-s time delay was implemented during MS sessions. Across all MS sessions, Tom needed all three levels of prompting. By his $15^{\text {th }} \mathrm{MS}$ session (Session 59 of Figure 5.4), little progress was observed and it appeared that Tom was becoming prompt dependent. Thus the 30 -s time delay followed by graduated guidance, as well as differential reinforcement was introduced. Tom had shown slight improvement during initial graduated guidance sessions, but no improvement with least-most prompting; it was therefore thought that reverting back to graduated guidance with a greater time delay and differential reinforcement might increase opportunities for independent responding. With this new prompting procedure, Tom demonstrated $100 \%$ correct requesting at his $22^{\text {nd }} M S$ session and achieved the acquisition criterion by his $24^{\text {th }}$ signing session (Session 87 of Figure 5.4). Anecdotally, throughout both SGD and MS sessions Tom demonstrated an increase in his spoken language. He often imitated the trainer's words surrounding the play activity and even spontaneously spoke several words and short phrases.

Throughout intervention Tom received seven device preference assessments. He consistently chose the SGD and used it correctly to make a request. On two occasions, however, Tom did not make a choice for MS or SGD. During post-intervention preference assessments Tom received a total of four choice opportunities. He chose the SGD on each occasion and achieved $100 \%$ correct requesting with it. Tom maintained a high level of correct requesting using the SGD during the one month follow-up and chose to use it on each device preference assessment. His MS use ranged from 0 to $40 \%$ correct. At the six month follow-up, Tom's preference for, and correct use of, the SGD (both 100\%) maintained. Correct use of MS dropped completely to $0 \%$. Overall Tom received 19 preference assessments (Figure 5.3) and chose the SGD the majority of the time $(89 \%)$. 


\section{Zac}

Zac made little progress to request with either AAC option. With the implementation of mass training trials (Session 57 of Figure 5.4), Zac achieved criterion using the SGD on his $14^{\text {th }}$ SGD session (Session 77 of Figure 5.4). He failed to reach criterion during MS sessions. Throughout intervention Zac received five device preference assessments. He chose the SGD on each of these assessments. Once he was able to use the SGD independently, he was also able to request to play correctly on these preference assessments. Zac did not progress to the post-intervention phase due to his failure to acquire use of the MS option.

Zac maintained a high level (80-90\%) of correct requesting with the SGD, but he did not make any correct requests with MS during the one month followup. Despite not being able to make a correct request with it, Zac did choose the MS option on one preference assessment opportunity, but chose the SGD on the other three assessments. Zac maintained higher levels of correct requesting (90$100 \%$ ) and preference (100\%) for SGD during the six month follow-up. His MS remained at $0 \%$ correct. Overall, Zac received 13 AAC preference assessments (Figure 5.3) during which he chose the SGD 92\% of the time.

Eli

Eli achieved criterion of requests for toys using the SGD on his $3^{\text {rd }}$ SGD session (Session 63 of Figure 5.4). However, throughout the SGD intervention sessions he made many errors, repeatedly pressing all three icons (SNACK, PLAY, and SOCIAL INTERACTION) on the SGD. Eli achieved criterion for requesting toys using MS on his $10^{\text {th }} \mathrm{MS}$ session (Session 84 of Figure 5.4). During the intervention phase he received two device preference assessments each time choosing the SGD and using it correctly to request to play with toys. During the post-intervention preference assessments Eli received a total of four choice opportunities and chose the SGD all four times in which he requested at 90 to $100 \%$ proficiency.

Eli's performance dropped for both the SGD (30 to 50\% correct requesting) and MS (10\% correct requesting) during the one month follow-up. He chose the SGD on $100 \%$ of choice opportunities using it with $100 \%$ proficiency on three out of four device preference assessments. As with the other participants, 
Eli's performance increased during the six month follow-up, with 40 to $60 \%$ correct requesting with the SGD and 0 to $20 \%$ correct requesting with MS. Although he chose the SGD on $100 \%$ of opportunities, Eli used it correctly on only one of the four occasions. Overall Eli received 16 opportunities to choose between the SGD and MS option (Figure 5.3) during which he always chose the $\operatorname{SGD}(100 \%)$.

\section{Discussion}

All four participants learnt to make augmented requests in this study and also appeared to demonstrate a preference for using either an iPod®-based SGD or MS. Specifically, three (David, Tom, and Eli) of the four boys reached criterion on use of both communication options to request preferred stimuli. The fourth boy (Zac) reached criterion for SGD use, but did not learn to use MS. Tom, Zac, and Eli demonstrated a preference for using the SGD, while David exhibited a slight preference for using MS. These results support findings from previous research suggesting that students with DD can learn to use various AAC systems for functional communication and will often demonstrate a preference for one communication device over another (Chapter 3 ). Moreover, most of the children assessed to date in this and the previous studies reviewed in Chapter 3 showed a preference for using SGDs over PE (or MS), although it is important to note that studies comparing SGDs and MS are rare. In fact, the present study appears to be the only one to date that has directly compared acquisition of, and preference for, SGDs versus MS.

However, while results from this systematic review of the preference literature (Chapter 3 ) and results from other studies comparing acquisition of various modes of AAC (e.g., Bock et al., 2005; Gregory et al., 2009) highlight few differences in terms of how quickly children learn to use each communication system, outcomes from the present investigation indicated differences in terms of how rapidly participants learnt to use each AAC option. Specifically, three of the four participants learnt to use the SGD (also their preferred communication option) more quickly than MS. A possible explanation for this difference in findings is that the majority of the previous research did not include a comparison between SGD and MS (e.g., Bock et al., 2005; Gregory et al., 2009). Of the few studies (lacono \& Duncum, 1995; lacono et al., 1993; Sigafoos \& Drasgow, 2001) 
that did compare SGD with MS, the results also showed slightly better performance with the SGD option. Similarly, results from research comparing acquisition of PECS and MS indicate that PECS was learnt at a faster rate than MS (Adkins \& Axelrod, 2001; Rotholz et al., 1989; Tincani, 2004), but it is arguable whether these differences were clinically significant. In this and previous studies, SGD use could have been acquired faster because it might be more difficult to teach MS use, rather than it being inherently more difficult for children with DD to learn.

The findings of the present study, when considered in light of previously reviewed studies (Chapter 3 ), suggest that there may not be one single mode of $A A C$ that is best for children with DD. Thus decisions as to which AAC option to implement might be based upon an assessment of the individual user's unique abilities, needs, and their preferences for different AAC options (Sigafoos \& Drasgow, 2001). A key aspect of the present study was the assessment of each child's preference for the two AAC options using a structured choice-making protocol (e.g., Sigafoos, 1998). In previous preference research (Sigafoos et al., 2005; Son et al., 2006; Soto et al., 1993), it has been argued that because participants had equal exposure to and achieved equal proficiency with each communication system prior to implementing the choice assessment, they were able to make an informed choice, and that preference for one device over another was not due to differences in performance. However, by utilising this post-hoc approach, it remained unclear when a child's preference for an AAC option might first emerge and why children might prefer one AAC option over another. It was also unclear whether preferences led to improved therapeutic outcomes. By incorporating preference assessments into the initial stages of the intervention, the present study was able to determine when preferences began to emerge and whether preference might have influenced acquisition and maintenance.

Along these lines, the present results indicated that for three of the four participants (Tom, Zac, and Eli), preference was evident during the early stages of intervention and appeared to remain stable during the study. For David, however, preference seemed to change as he gained proficiency with each communication option. Specifically, David learnt MS rapidly and initially 
demonstrated a preference for this communication option. However, after learning to use the SGD, he then started to choose this communication option.

The results also suggested that participants were more proficient at using their preferred AAC option or perhaps it should be said that they preferred the option with which they were more proficient. Furthermore, maintenance of newly acquired communication skills was better when participants used their preferred option. These results provide the first empirical evidence to suggest that allowing individuals with DD to determine which communication option they would like to use might positively influence progress in learning to communicate. This is supported by the finding that effect sizes were greater for the participant's preferred communication option. These findings support the argument by Soto and colleagues (1993) that AAC acquisition is interconnected with choice-making and preference.

However, similar to the literature pertaining to general choice-making and assessment of preference in individuals with DD (e.g., Stafford et al., 2002), preference did change over time for one participant (David), verifying the idea that preference should be reassessed at regular intervals. Because preferences can vary it has also been suggested that an individual's preference, or the 'magnitude of preference', for one AAC option over another should be interpreted with caution (Chapter 3). It is unclear how many choice opportunities are required to demonstrate a preference for one mode of communication over another. There is not yet any firm empirical basis for setting the percentage of selections from which an item can be considered preferred. Future research should investigate this issue.

Results from the present study suggest that the choice-making paradigm (e.g., Sigafoos, 1998) utilised in the preference research to date might be effectively extended to assess preferences for aided (SGD) versus unaided (MS) AAC. Prior to this study, it was unknown whether using a photo/diagram of a person signing would be a viable means of representing the MS option. David did select the MS option the majority of the time suggesting that at least one participant did understand that the photo of the trainer signing represented the MS option, enabling him to indicate a preference for an unaided mode of AAC. Future research is required to further investigate the validity of this approach, 
especially considering it could be argued that the SGD and MS options were not functionally equivalent. While the SGD option included symbols for SOCIAL INTERACTION, SNACK, and PLAY, the MS option only had two photos to represent SNACK and PLAY. Furthermore, in order to compare two conditions on symbol learning, it is crucial to equate the sets of symbol-referent associations for SGD and MS for the degree of iconicity and for receptive understanding of the referent (Schlosser, 2003c). Linked to this, it has been argued that the response effort required for each communication system may influence preference and intervention outcomes (Ringdahl et al., 2009; Winborn-Kemmerer et al., 2009). Although signing to request to eat/play was considered comparable to using the SGD, this decision was based only upon informal methods of creating equivalency in terms of performance difficulty (Schlosser, 2003c). However, the two systems might not be comparable in terms of cognitive demands. Indeed, it has been argued that graphic symbols of the type used with the SGD are less demanding on working memory because only recognition is needed, whereas the MS option requires the use of recall memory (lacono et al., 1993). This could be one possible reason why some participants might demonstrate a preference for SGD over MS (lacono \& Duncum, 1995).

A further potential problem of the present study is that a MS response could be emitted during SGD sessions, but not vice versa because the SGD was not available during MS sessions. This could have meant that correct MS responses were put on a more intermittent schedule (correct MS responses resulted in reinforcement only some of the time, while correct SGD responses resulted in reinforcement $100 \%$ of the time) relative to correct SGD responses, thereby weakening the teaching contingencies and potentially influencing both preferences and rapidity of acquisition. Despite this possibility, the majority of participants (David, Tom, and Eli) did learn to use both MS and SGD for functional communication suggesting that any disruption from intermittent reinforcement of the MS option was minimal and short-lived. Overall, the results of the intervention phase, showing acquisition of SGD and MS use in most cases, are consistent with previous research (Rispoli et al., 2010; van der Meer \& Rispoli, 2010; Wendt, 2009) showing that systematic instructional procedures, including graduated guidance, time delay, and contingent reinforcement 
procedures, can be effectively applied to teach requesting for preferred stimuli using both unaided and aided AAC (Duker et al., 2004).

While David and Eli demonstrated rapid acquisition of both the SGD and MS, Zac failed to show any progress during intervention for MS and struggled to learn the SGD. Adaptation of the instructional techniques included the introduction of mass training trials. With this procedure in place Zac rapidly learnt to use the SGD, but failed to learn MS. His lack of progress with the MS option did not appear to be a motivational issue in that he did consistently select and play with preferred stimuli. In addition, it did not appear to be due to an inability to physically perform the hand gestures required to make the manual sign because he did independently request to play using MS on a number of occasions. Anecdotally, when Zac was prompted to make the sign for PLAY he also often attempted to verbalise the word play. As mentioned earlier, treatment outcomes for learning alternative forms of communication may vary if the new response topography is more demanding than the individual's original form of communication (Winborn-Kemmerer et al., 2009). Perhaps learning the sign for PLAY required significantly more effort for Zac than attempting to verbalise the request.

Adaptations to the instructional procedures, including least-most prompting and later an increased time-delay with graduated guidance and differential reinforcement did lead to acquisition of the MS option for Tom. This highlights the importance of not only implementing well established teaching procedures, but also modifying these techniques to suit the needs of each individual (Linscheid, 1999). Results also support preliminary evidence in the instructional literature on teaching the use of a new iPod®-based SGD. Similar to this previous research (Kagohara et al., 2010; van der Meer, Kagohara, et al., 2011), two of the participants (David and Zac) in the present study did show some initial difficulty in learning to touch the target symbols on the SGD with sufficient finesse to activate speech output. Fortunately, with practice, both David and Zac became proficient with using the iPod®-based SGD.

While using the behavioural strategies described above often leads to rapid acquisition of functional communication, several problems can emerge following acquisition of an initial requesting repertoire (Sigafoos, Ganz, O'Reilly, \& 
Lancioni, 2008). One such problem, which was evident for David and Eli, was the development of perseverative requesting, which refers to making repeated requests, even after being informed to wait for the requested item or when the item is not being offered (Sigafoos et al.). David initially used MS repeatedly even during SGD sessions, which Cannella-Malone and colleagues (2009) suggested could be an indication of preference for a particular AAC device. However, we ignored signing when it was used in an incorrect context and this appeared to be a useful part of the training program for David because he eventually developed proficiency with the SGD and his perseverative signing diminished. He also began to choose the SGD during the device preference assessments. Eli, on the other hand, developed a tendency to repeatedly press the icons on the SGD resulting in constant voice-output. Observations of this behaviour suggested that this might have been automatically reinforced by the resulting synthesised speech output.

Because Eli repeatedly pressed non-target symbols on the SGD, it could be argued that he did not have good symbol discrimination abilities. However, for the most part Eli and the other participants did correctly request to play (eat) the items that were being offered, indicating good symbol discrimination abilities. Still, future research could implement additional manipulations to control for itembiased or position-biased responding, such as the procedures implemented by van der Meer, Kagohara, et al. (2011). They shifted the orientation of the SGD and alternated offering only toys or only snacks across sessions to test for the relevant symbol discriminations.

The results of the present study are limited in several ways and should be interpreted with caution. In line with previous research on assessing preferences for different AAC options (see Chapter 3), the present study focused on teaching a single communicative function (i.e., requesting). It therefore remains unclear whether preference will influence learning of other communication skills. Furthermore, because a verbal prompt (Let me know if you want something.) was used to initiate requesting opportunities, it is possible that the participant's responses were at least partially under the control of the speech of the communication partner. This means that the participants might not have acquired the ability to request items more spontaneously. Future interventions could be 
improved by teaching more spontaneous communication (Sigafoos, Drasgow, et al., 2009). The fact that only a limited communicative repertoire was taught and that generalisation data to other communicative forms, contexts and people, were not collected are further limitations. Lastly, preferences across a wider range of AAC options (e.g., PECS, SGD, and MS) have yet to be investigated. Since these are the three main AAC modes taught to children with ASD and other DD, Study 2 of the present thesis, presented in Chapter 6, simultaneously compared all three options in terms of acquisition, preference, and maintenance. 


\section{CHAPTER 6}

\section{STUDY 2}

\section{Comparing Three Augmentative and Alternative Communication Modes for Children with Developmental Disabilities ${ }^{5}$}

\section{Aims and Hypothesis}

The present study was designed to systematically replicate and extend Study 1 (Chapter 5) by including more participants and assessing preferences for, not only SGD and MS, but also PE, thereby providing some of the first empirical data comparing acquisition of all three common modes of $A A C$ for individuals with ASD and related DD. Based on previous research and theoretical perspectives (Chapter 3 ) and the results of Study 1 (Chapter 5 ) it was hypothesised that the four children participating in the present study would show a preference for using one AAC mode over the other two, that these preferences would vary across children, and that the children would learn to make general requests (e.g., I want to play) more quickly with their most preferred AAC mode.

\section{Method}

\section{Participants}

Four children were recruited due to their severely limited speech from a childcare centre for children with DD. All four participants met the following criteria: (a) diagnosis of intellectual/developmental disability or ASD, (b) less than 18 years of age, (c) very limited or no communication skills as determined by an age equivalency of 2 years or less on the communication domain of the Vineland - Z, Dutch edition (Sparrow, Balla, \& Cicchetti, 2003), (d) no auditory or visual impairments that would interfere with the use of $A A C$, and (e) sufficient motor skills to operate/perform the motor actions required to use each of the three AAC modes.

Joe. Joe was a 12-year-old male diagnosed with ASD. On the Vineland $Z$ (Sparrow et al., 2003), Joe received age equivalencies of 1:2 (years:months)

\footnotetext{
${ }^{5}$ An article based on this study has been published in Journal of Developmental and Physical Disabilities: van der Meer, L., Didden, R., Sutherland, D., O'Reilly, M., Lancioni, G., \& Sigafoos, J. (2012). Comparing three augmentative and alternative communication modes for children with developmental disabilities. Journal of Developmental and Physical Disabilities, 24, 451-468 (see Appendix F).
} 
for communication, 1:4 for daily living skills, and 1:1 for socialisation. Joe did not have any spoken language, but made sounds that were presumed to be his way of expressing how he was feeling (e.g., happy versus sad). He also appeared to communicate his wants and needs by taking a person's hand and leading them to an object. Joe's teachers used a picture communication board to explain routine activities for the day (toilet, food and drink, gym, bus, free choice, and outside play). Teachers had also introduced him to MS for receptive language. He did not have any prior experience with SGDs, PE, or MS as a communication mode for requesting access to preferred objects. Joe's fine and gross motor skills appeared to be adequate for his chronological age. He demonstrated frequent stereotypical and repetitive behaviours, such as flapping small toys and pieces of paper in front of his eyes.

Sam. Sam was a 6-year-old male diagnosed with childhood disintegrative disorder and intellectual disability. He received age equivalencies on the Vineland - Z (Sparrow et al., 2003) of 1:2, 1:8, and 0:11 (years:months) for the communication, daily living skills, and socialisation domains, respectively. Sam did not have any spoken language. He would take a person's hand and lead them to objects to seemingly express his wants and needs. He also made sounds that were thought to be indications of disapproval. His teachers tried to use MS when communicating with Sam, but he did not appear to show any interest in using MS to make requests. As was the case for Joe, Sam did not have any prior experience with SGDs, PE, or MS as a communication mode for requesting access to preferred objects.

Saskia. Saskia was a 10-year-old female with Angelman syndrome. She received an age equivalency of 1:4 (years:months) for the communication domain and 1:0 for both the daily living skills and socialisation domains of the Vineland Z (Sparrow et al., 2003). Saskia was able to speak several single words, mostly in the form of echolalia. She would often take people's hands to seemingly direct them to what she wanted, to open things, or to clap for her. Saskia had some experience with MS and was able to produce the signs for FINISHED and OPEN. She had no further experience with other forms of AAC. She exhibited difficulty with social interaction and engaged in stereotypic and repetitive behaviours. For example, she seemed more interested in adults and, although she appeared to 
enjoy watching other children play, she would push them away if they approached her. She also flapped keys and other objects in front of her eyes.

Nicky. Nicky was a 13-year-old female diagnosed with pervasive developmental disorder not otherwise specified (PDD-NOS). Nicky received age equivalencies on the Vineland $-Z$ (Sparrow et al., 2003) of 1:3, $1: 1$, and 1:3 (years:months) for the communication, daily living skills, and socialisation domains respectively. She was able to verbalise several single words, but generally only made babbling sounds. She had previously received MS training and was able to produce several signs, including OPEN, EAT, and CIRCLE TIME. Nicky appeared to understand symbols from her daily picture communication book and from routine activities. She was able to match picture cards and had received one year of PECS training, but reportedly had made little progress. She had received no further training in the use of $A A C$ for requesting preferred objects. Nicky was able to maintain good eye contact and seek social contact, but she was said to be very excitable and did not seem to understand social boundaries. She was not able to play cooperatively with other children. Nicky often cried in an apparent attempt to gain attention. Nicky's fine motor skills were adequate for her developmental level. She was able to walk on her own, although she was hypotonic.

\section{Setting and Intervention Context}

Participants were recruited from a Dutch childcare centre for children with DD. The procedures related to this study were conducted in a small therapy room across the hall from the children's main classrooms. Sessions occurred during morning and afternoon snack/leisure activities. The procedures were implemented in a one-to-one context consisting of the trainer (author) and one participant at a time. No one else was present in the room, other than one or two reliability and procedural integrity observers (who sat in the corner) on some occasions. All instructions/interactions with the participants and responses programmed on the AAC systems were in the Dutch language.

\section{Preferred Stimuli}

Stimuli that the children seemed to prefer, and which would be appropriate for them to request during a snack or leisure activity, were identified by a systematic two-stage stimulus preference assessment (Green et al., 2008). Stage 
1 of the preference assessment involved an indirect assessment in which teachers were asked to list foods, sensory stimuli, and toys that the participants appeared to enjoy and would be appropriate for the intervention.

For Stage 2, three to six of the most preferred food or play stimuli were then selected for a direct stimulus assessment. The direct preference assessment for Joe focused on identifying preferred foods because his intervention occurred during a snack activity and his teachers reported that he seemed to be highly motivated by snack foods. The direct preference assessment for Sam, Saskia, and Nicky, in contrast, focused on identifying preferred toys because their intervention occurred during a play activity and their teachers had concerns about using food reinforcers. Stage 2 involved the simultaneous presentation of multiple items, without replacement (DeLeon \& Iwata, 1996; Duker et al., 2004). Each participant was presented with an array of items from Stage 1 (random placement) and allowed to select one item. Items were not replaced once they had been selected, thereby eliminating the chance of the participant choosing only one or a few items, as well as allowing the trainer to develop a rank order of items in terms of preference. The top three food items for Joe and top three play items for Sam, Saskia, Nicky were identified by calculating a rank order of the percentage of times that the stimuli were selected. Across two to three sessions, each item was offered a total of nine times. Rank orders were calculated using the formula: Number of Selections/Number of Offers $x 100 \%$.

Preferred stimuli for Joe included 'skittles' lollies (75\%), 'tumtum' lollies (33\%), and potato chips (32\%). Preferred stimuli for Sam included a puzzle $(60 \%)$, venting ball (36\%), and windmill (33\%). Preferred stimuli for Saskia included a musical toy (82\%), keys and lanyard (39\%), and bubbles $(31 \%)$. Preferred items for Nicky included a tea set (69\%), dolls (56\%), and a mirror (32\%).

\section{SGD}

Participants were taught to request preferred toys or snacks using an Apple iPod Touch ${ }^{\circledR}$ with Proloquo2Go ${ }^{T M}$ software (Sennott \& Bowker, 2009). The iPod was placed inside an iMain $G o \circledast 2$ speaker case to increase sound amplification. The iPod Touch $\circledast$ was configured to show a single page containing 
two graphic symbols $(2.5 \times 2.5 \mathrm{~cm})$, representing requests for $S N A C K S$ and PLAY (Figure 6.1). The messages were programmed in Dutch. Touching each symbol activated corresponding synthetic speech-output (i.e., "I want something to eat.", and "I want to play.").

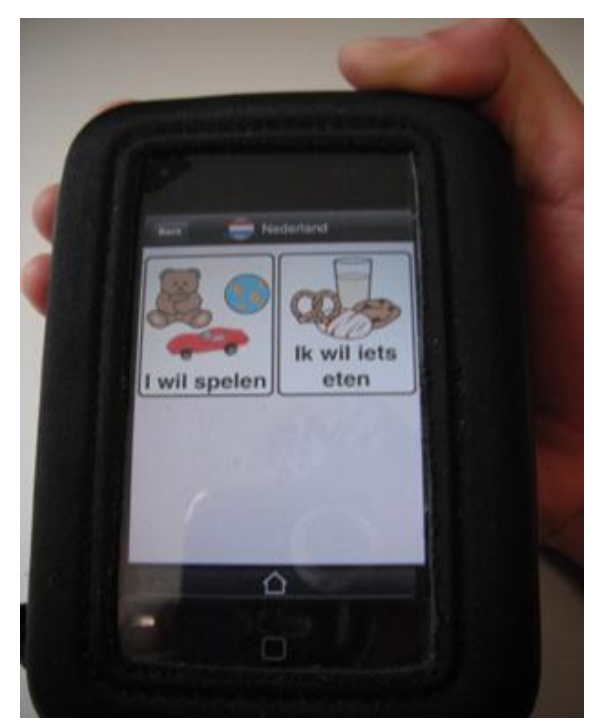

Figure 6.1. Photograph of the iPod Touch ${ }^{\circledR}$ inside the iMainGo ${ }^{\circledR} 2$ Speaker Case with the Visual Display of the Graphic Symbols for PLAY and SNACKS for Study 2.

\section{PE}

Participants were also taught to request their preferred toys or snacks using PE. Three $(6 \times 6 \mathrm{~cm})$ symbols from the PECS 2009 Dictionary (Pyramid Educational Products, 2009) were affixed with Velcro ${ }^{\mathrm{TM}}$ to a $19 \times 13 \mathrm{~cm}$ card. One symbol contained a coloured line drawing showing two hands reaching out and the words I WANT. The second symbol consisted of a coloured line drawing of various different toys and the words TO PLAY. The third symbol consisted of a coloured line drawing of various snack items and the message SOMETHING TO EAT. All words were written in Dutch. The symbols were randomly allocated to the six $(6 \times 6 \mathrm{~cm})$ panels of the card (Figure 6.2). 


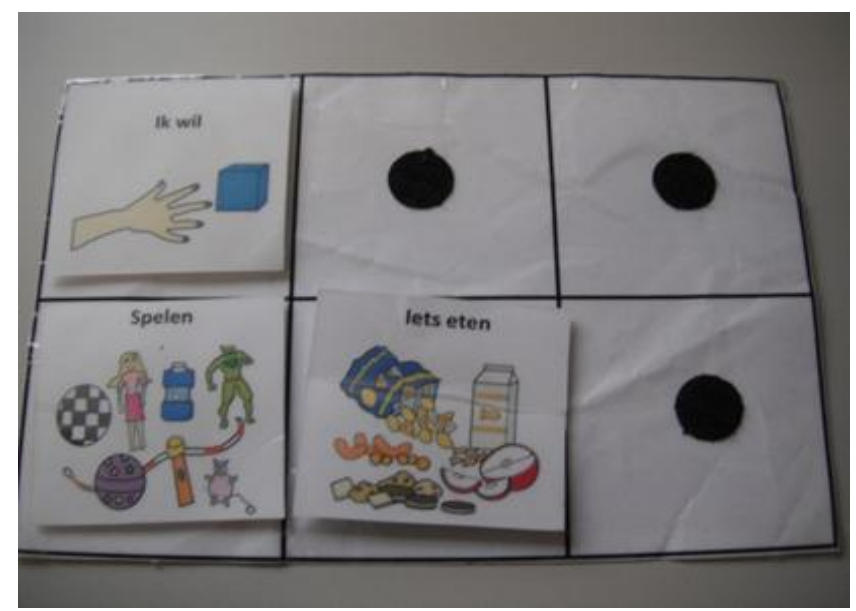

Figure 6.2. Photograph of the PE Communication Option with Graphic Symbols for I WANT, TO PLAY, and SOMETHING TO EAT for Study 2.

\section{MS}

Participants were taught to request their preferred toys or snacks using signs from the Dutch sign language system for children (Nederlands Gebarencentrum, 2006). Participants were taught the sign for SNACK or PLAY. The MS option was represented by a laminated photograph $(15 \times 8 \mathrm{~cm})$ of the trainer making the hand formations for the signs for SNACK and PLAY (Figure 6.3).
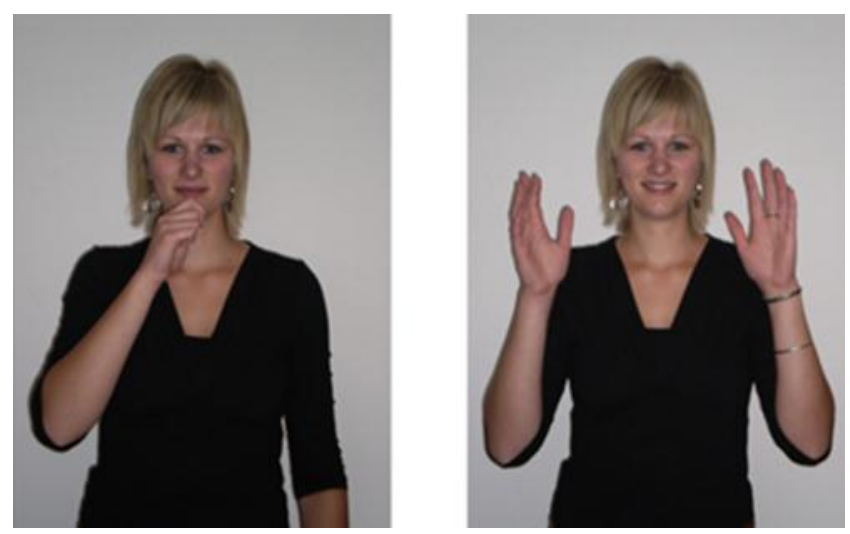

Figure 6.3. Photograph of the Trainer Making the Signs for EAT and PLAY to Represent the MS Communication Option for Study 2.

\section{Response Definitions and Measurement}

For SGD use, correct responding was defined as independently (without a gestural or verbal prompt) touching the symbol on the screen of the SGD to 
activate the corresponding speech output in exchange for a desired item from the trainer. MS was defined as independent (without a gestural or verbal prompt) hand gestures to produce correct signs in exchange for a desired item from the trainer. Independent approximations of the sign (e.g.,rotating hands once or several times for play, instead of exactly twice) were counted as correct. For PE, participants were required to independently (without gestural or verbal prompt) place the I WANT and corresponding (SNACKS or PLAY) symbols - depending on whether they were requesting snacks or play - onto the two panels $(6 \times 6$ $\mathrm{cm})$ provided on a separate $(21 \times 7 \mathrm{~cm})$ card in exchange for the desired item from the trainer.

The percentage of correct responses (requests) was calculated for each session. Each session consisted of 10 offers to request snack or play items. The SGD target response for Joe was touching the SNACK symbol on the SGD to activate the message "I want a snack." His target response for PE was placing the I WANT and SOMETHING TO EAT symbols on the separate card. The PLAY symbol (SGD and PE) was intended as a distracter. Joe's target response for MS was to produce the manual sign for SNACK. The PLAY sign (on the laminated card) was intended as a distractor. The SGD target response for Sam, Saskia, and Nicky was touching the PLAY symbol on the SGD to activate the message "I want to play." Their target response for PE was placing the I WANT and TO PLAY symbols on the separate card. The SNACK symbol (SGD and PE) was intended as a distracter. The MS target response for Sam, Saskia, and Nicky was to produce the manual sign for PLAY. The SNACK sign (on the laminated card) was intended as a distractor.

\section{Experimental Design}

The study included the following phases arranged in a multiple-probe across participants design (Horner \& Baer, 1978): Baseline, intervention, preference assessment (throughout intervention, post-intervention, and followup), post-intervention, and follow-up. An alternating-treatments design (Barlow \& Hayes, 1979; Barrett \& Sisson, 1987) was embedded within each phase of the multiple-probe to compare children's performance with the SGD, PE, and MS options. 


\section{Session Schedule}

Two to four sessions were conducted five days per week. Each session lasted about 10 min and consisted of 10 discrete trials. The AAC option available (i.e., SGD, PE, or MS) was counterbalanced across sessions to prevent order effects (Kennedy, 2005). For all sessions, the participant and trainer were seated next to each other at a table with one or two additional reliability and procedural integrity observers seated nearby.

Once a participant showed an increase in requesting behaviour above the level established in baseline for three consecutive sessions with at least one of the AAC options, training commenced with the next participant. Training was first provided to Joe, then Sam, Saskia, and finally Nicky. Training was provided in this order in accordance with results from the baseline phase (i.e., the participant with the most stable baseline commenced intervention first). Once a participant reached criterion for one $\mathrm{AAC}$ condition (i.e., $80 \%$ correct requesting across three consecutive sessions for each AAC option), maintenance probes were initiated with that system while the other communication systems continued to be taught using the intervention procedures. One maintenance session with the acquired AAC device was conducted after three sessions with each of the AAC options still being taught.

\section{Procedures}

Because participants were considered to be at the beginning stages of AAC intervention, they were taught to make general requests for either snacks or toys from which they could select one highly preferred item after each request. However, to ensure some level of symbol discrimination, the distractor symbols/signs were included on the AAC options. If participants requested a snack when they were undertaking training to request a toy or vice versa, the trainer explained: We are learning to request toys (snack) at the moment, you can request a toy (snack) another time. It was considered natural to provide them with this feedback when they activated the non-target symbols/produced the nontarget signs, but not to reinforce it with preferred tangibles. Similarly, producing MS to request snacks or toys during SGD or PE sessions was ignored in order to bring the use of each device under stimulus control. 
Baseline. During baseline, a tray containing three different snack (play) items was placed on the table in view, but out of the participants' reach. The SGD, PE, and MS option (represented by the photograph of the trainer making the two signs) were randomly placed on different sides of the table for each baseline session. Each session involved 10 discrete trials for snacks or toys. The session began with the trainer telling the participant: Here is a tray of snacks (toys), let me know if you want something. After $10 \mathrm{~s}$, the trainer moved the tray within reach and allowed the participant to take one item. This 10-s fixed time schedule of reinforcement was provided to ensure continued motivation to participate in sessions. When offering snacks, participants were allowed to select one item from the tray, which was then replenished before the next offer. When offering the tray of toys, participants were allowed to select one toy and play with it for approximately $30 \mathrm{~s}$ before it was returned to the tray. SGD, PE, and MS responses were recorded, but had no programmed consequences.

Intervention. This phase was conducted in a discrete trial format until participants reached criterion (i.e., $80 \%$ correct requesting across three consecutive sessions for each AAC option). The SGD, PE, or the MS option was placed on the table (counterbalanced across trials) within reach of the child in accordance with the alternating treatments design. Each trial consisted of the trainer pointing to a tray of snacks (toys) and saying: Here's a tray of snacks (toys). Let me know if you want something. Training involved a 10-s time delay between the verbal cue (i.e., Let me know if you want something.) and the use of graduated guidance to prompt a correct request. Graduated guidance involved use of the least amount of physical guidance necessary to ensure the child made a correct request, while simultaneously explaining the required response (e.g., Press PLAY to ask to play with a toy. or Move your hand to your mouth to make the sign for EAT. or Put the I WANT and PLAY pictures on the velcro strips). Immediately after the child had used the SGD to produce the correct synthesised speech output, or had placed the appropriate symbols on the Velcro strips, or had made the correct manual sign, the trainer moved the tray containing the snacks (or toys) within reach of the participant. The participant was allowed to select one item from the tray and consume the chosen snack or play with the chosen toy for about $30 \mathrm{~s}$. After this, the next trial was initiated. 
Procedural modifications. Joe did not reach acquisition for each communication system during the initial intervention phase so he received a modified intervention. The modification was developed in response to what appeared to be a problem in teaching him to discriminate among the different symbols. Therefore, the PLAY symbol was removed from the SGD and PE options (see the 1 Symbol phase of Figure 6.5). The only icon displayed on the SGD screen was therefore the SNACK icon, which was also enlarged to fit the entire screen. Joe was only required to press this icon to activate the voice-output in order to make a correct request for a preferred snack item. For the PE option, he no longer had to discriminate between the SNACK and PLAY symbols. For a correct request he had to place the I WANT and SOMETHIG TO EAT symbols onto the two locations provided on the separate card in exchange for the desired item from the trainer.

Sam also failed to learn how to use the SGD and MS communication options during the initial intervention sessions. Therefore, for SGD and for MS (see the 20 s Time Delay \& Differential Reinforcement phase of Figure 6.5), the procedures changed to using a longer (20 s) time delay followed by graduated guidance, as well as differential reinforcement (where Sam was only given the opportunity to play if he independently used the SGD or MS to request to play). Prompted trials were not reinforced. Because little progress was evident with these changes, a $0 \mathrm{~s}$ time delay was then implemented and immediate reinforcement was reintroduced (See the $0 \mathrm{~s}$ Time Delay and Differential Reinforcement phase of Figure 6.5). That is, Sam was immediately prompted to make a correct request and then given access to the tray. However, for the MS option Sam appeared to become dependent on the trainer immediately prompting a correct response. He did not attempt to make the sign for PLAY and so for this reason, a $10 \mathrm{~s}$ time delay with reinforcement was reintroduced (10 s Time Delay and Differential Reinforcement phase of Figure 6.5).

AAC Preference Assessments. These assessments were undertaken to determine if participants would show a preference for using one of the three AAC options. They were undertaken after every sixth intervention session (i.e., after two sessions for each AAC option). During each preference assessment, the SGD, PE, and MS options were presented (randomly) at different positions on the 
table. While pointing to each option, the trainer asked the participant: Which communication option would you like to use? The SGD, PE, or MS? The child had $10 \mathrm{~s}$ in which to make a choice by touching one of the options. Once a choice was made, the trainer initiated one requesting opportunity with the chosen $A A C$ option before reverting back to initiating requesting opportunities with the AAC device that was scheduled to be used for the session. If the child did not choose an option within $10 \mathrm{~s}$, the device preference assessment was terminated and training continued with the AAC option that was scheduled for use in that session.

Post-intervention. Once the participant reached criterion for each AAC device, post-intervention preference assessments were introduced. These were identical to the previously described preference assessments, except that once an AAC option had been chosen, the participant continued to request preferred items using the chosen communication method for the entire 10-trial session.

Follow-up. Six follow-up sessions were conducted two weeks following post-intervention for Joe (Session 88 of Figure 6.5) and Saskia (Session 85 of Figure 6.5). Participants did not use either communication option during the break. Because Sam and Nicky did not complete all phases of the study due to time constraints, they did not receive any follow-up probes. Procedures for followup were identical to the intervention phase, except no prompting occurred and reinforcement was contingent upon a correct request. One AAC preference assessment was implemented before each follow-up session.

\section{Inter-Observer Agreement}

The trainer collected data on the frequency of correct requesting, the level of prompting required during intervention for each trial, as well as which communication mode was selected during the AAC preference assessments. To assess the reliability of the trainer's data collection, an independent observer also collected data on the frequency of requesting, level of prompting, and communication mode chosen. For each session, percentages of agreement between the independent observer and the trainer were calculated using the formula: Agreements/(Agreements + Disagreements) x 100\%. Inter-observer agreement data were collected on $28 \%$ of all sessions and ranged from 80 to $100 \%$ with a mean of $99.2 \%$. 


\section{Procedural Integrity}

To assess procedural integrity, the independent observer used a checklist of the procedures and recorded whether the trainer had correctly implemented each procedural step in its proper sequence. Procedural integrity was assessed on $28 \%$ of all sessions and ranged from 85 to $100 \%$ correct implementation of the procedural steps with an overall mean of $99.8 \%$. A second independent observer collected inter-observer agreement data on $7 \%$ of these integrity checks with $100 \%$ agreement.

\section{Results}

Figure 6.4 provides a summary of the results from the AAC preference assessments conducted during intervention and subsequent phases. Figure 6.5 shows the percentage of correct requests during each session/phase of the study and for each of the three AAC modes. In baseline (Figure 6.5), none of the participants ever used MS or PE to make the targeted requests. Saskia and Nicky made one and two correct SGD-based requests, respectively during baseline.

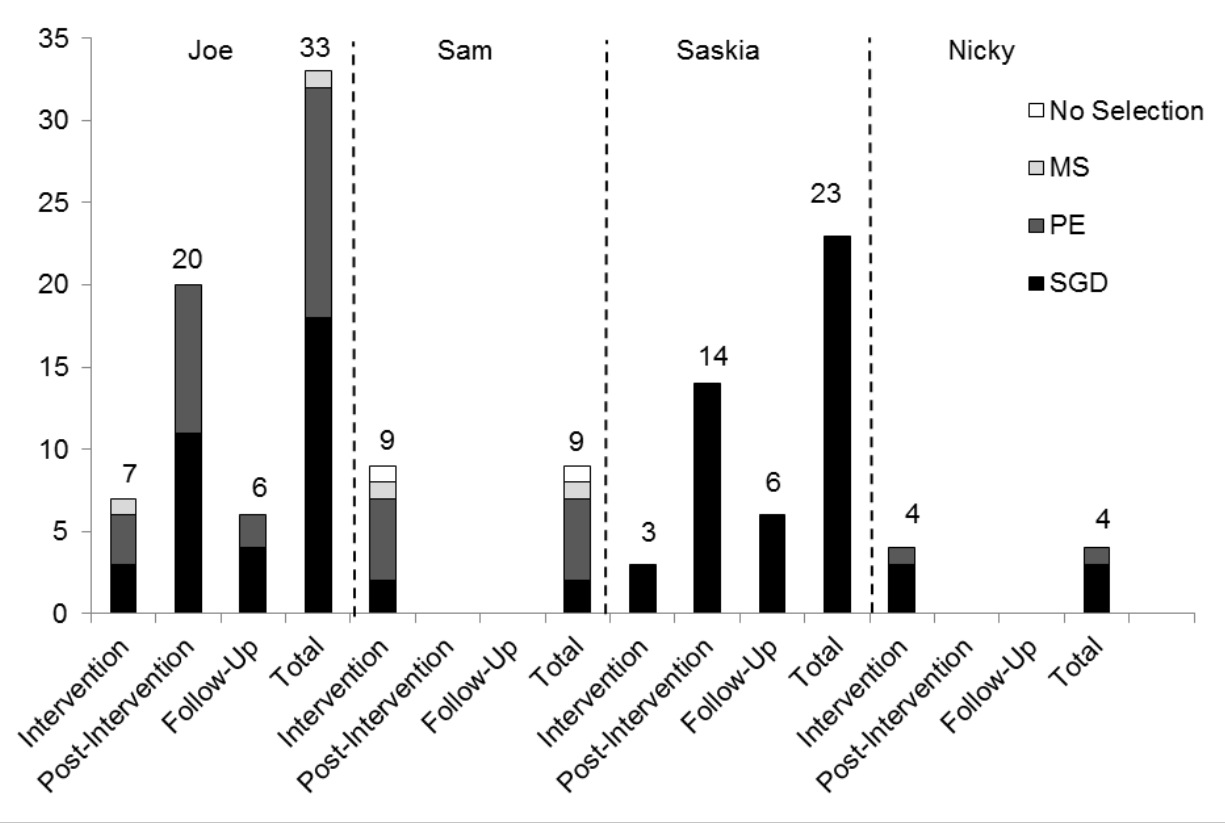

Figure 6.4. Results from the AAC Preference Assessments Depicting the Number of Times each Communication Option (SGD and MS) was Chosen across Phases and Participants. 


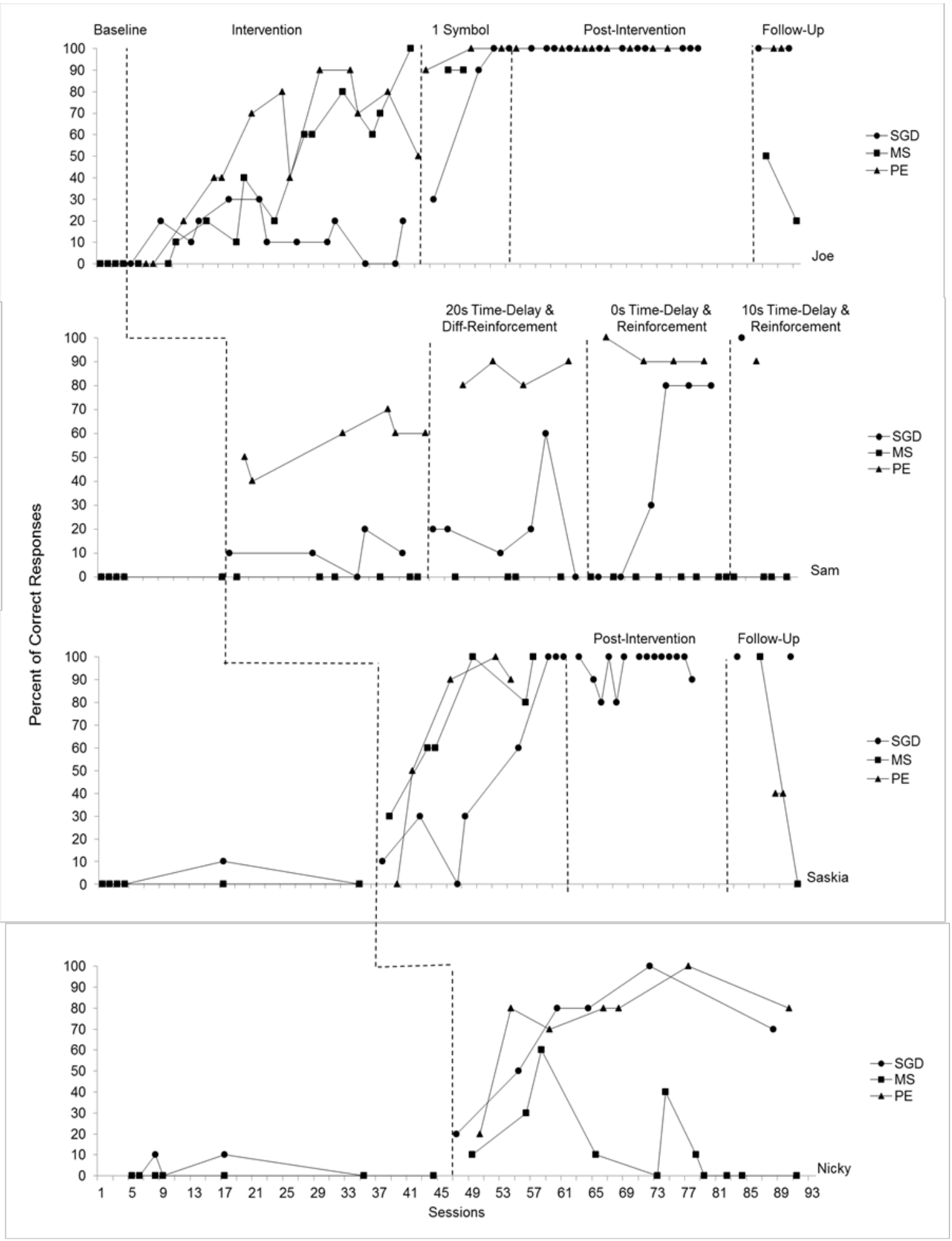

Figure 6.5. Percentage of Correct Requests with SGD and MS across Sessions for each Participant.

In order to calculate effect sizes NAP was used. NAP is used to determine the effectiveness of the intervention procedures on teaching target behaviours to the participants (Parker \& Vannest, 2009). Table 6.1 shows the resulting 
percentages of NAP. Effect sizes for each participant for treatment effects from baseline to intervention across communication options (SGD, PE, and MS) were calculated. Parker and Vannest (2009) explained that "large" effect sizes are generally equal to or greater than $80 \%$, but warned that these guidelines given by Cohen (1988) are from large $N$ social science research and should not be readily applied to other contexts. Nevertheless, "large" $(\geq 80 \%)$ effect sizes were found for all participants for intervention effects for SGD and PE. However, only two out of the four participants (Joe at $96 \%$ and Saskia at 100\%) received such high intervention effects for MS. Greater effects across participants were achieved for both SGD and PE communication options ( $M=94 \%)$ relative to $M S(M=62.5 \%)$. Similar to Study 1 (Chapter 5), high effect sizes for SGD and PE reflected participant's preferences for using SGD and/or PE. Descriptions of each participant's acquisition and preference are provided below.

Table 6.1. Baseline to intervention NAP Effect Sizes for SGD, PE, and MS for each Participant.

\begin{tabular}{llll}
\hline Participant & SGD & PE & MS \\
\hline Joe & $96 \%$ & $96 \%$ & $96 \%$ \\
Sam & $96 \%$ & $100 \%$ & $0 \%$ \\
Saskia & $84 \%$ & $80 \%$ & $100 \%$ \\
Nicky & $100 \%$ & $100 \%$ & $54 \%$ \\
\hline
\end{tabular}

NAP is rescaled to a $0-100 \%$ scale

Joe

When intervention was introduced, Joe reached the acquisition criterion for the MS option on his $15^{\text {th }}$ MS training session. Similarly, when intervention was introduced, and then modified by removing the distractor symbols, Joe achieved criterion with PE and SGD on his $16^{\text {th }}$ and $17^{\text {th }}$ intervention sessions, respectively. During the post-acquisition phase, Joe chose to use the SGD (55\%) more often than PE (45\%). Once chosen, he then used the selected option (i.e., either SGD or PE) with $100 \%$ proficiency. During follow-up, Joe maintained his level of correct SGD- and PE-based requests at 100\%, but his performance dropped to 20 and $50 \%$ correct for MS. Overall, Joe received a total of 33 
opportunities to choose between the SGD, PE and MS option (Figure 6.4) and he chose the SGD most frequently (61\%).

\section{Sam}

When intervention was introduced with Sam, he reached criterion with PE on his $9^{\text {th }}$ training session. When the intervention procedures were modified, he achieved criterion for SGD on his $17^{\text {th }}$ such session. However, even with additional procedural modifications, Sam did not achieve criterion for MS within the timeframe of this study. Sam did not progress to the post-intervention or follow-up phases due to his failure to acquire use of the MS option. During intervention, Sam received nine AAC preference assessments (Figure 6.4) and he chose PE most frequently (56\%).

\section{Saskia}

Saskia achieved criterion for PE-, MS- and SGD-based requests on her fifth, sixth, and eighth respective intervention sessions. During the postintervention phase, Saskia always chose to use the SGD and then used it with 80 to $100 \%$ proficiency. During follow-up, her performance maintained at $100 \%$ correct for the SGD, but decreased to $40 \%$ and $0 \%$ correct for the PE and MS modes, respectively. Overall, Saskia received 23 AAC preference assessments (Figure 6.4) during which she always chose the SGD.

\section{Nicky}

Nicky achieved acquisition criterion for SGD- and PE-based requests on her fifth and sixth respective intervention sessions. She showed an initial increase in the percentage of correct requests using MS, but failed to achieve criterion within the timeframe of the study and did not progress to the postintervention or follow-up phases. Across her four AAC preference assessments conducted during intervention (see Figure 6.4), she chose the SGD three times (75\%).

\section{Discussion}

The present study extends the findings of Study 1 (Chapter 5) by comparing acquisition of three common modes of AAC, namely SGD, PE, and $M S$. The findings suggest that the systematic instructional procedures used for each AAC option (Duker et al., 2004) were largely effective in teaching each 
participant to use at least two of the three AAC options. Furthermore, a key aspect of the study was to assess preferences for one mode of communication over the others throughout the intervention process, allowing participants some degree of self-determination with respect to AAC modes (Sigafoos, 2006). Specifically, two participants (Joe and Saskia) reached criterion for use with each communication option and demonstrated a preference for using the SGD. The other two participants (Sam and Nicky) reached criterion for SGD and PE, but not MS. Nicky exhibited a preference for using the SGD, while Sam demonstrated a slight preference for using PE.

The findings support those of previous studies suggesting that students with DD can learn to use a SGD, PE, and MS for functional communication and that many will also indicate a preference for using a particular communication system (Chapter 3; Study 1 - Chapter 5 ). The findings also provide further evidence indicating that most of the children assessed to date appear to show a preference for using SGD over PE and MS (Chapter 3; Study 1 - Chapter 5), although the present study appears to be the only one to date that has compared acquisition of, and preference for SGD, PE, and MS.

While all of the participants learnt to use PE and SGD, Sam and Nicky failed to reach criterion for MS, even with modifications to the intervention process. This finding could suggest that MS communication is more difficult for some children to learn or that the instructional procedures used in the present study were better suited for teaching use of the SGD and PE options. With respect to the first possibility, lacono and colleagues (lacono \& Duncum, 1995; lacono et al., 1993) suggested that graphic symbols, such as those used for the SGD and PE options in this study, are less demanding on children's working memory because only recognition memory is needed, whereas MS requires the use of recall memory. This could be one reason why MS is sometimes learnt at a slower rate than other AAC systems and this might also explain some of the patterns with respect to preferences for SGD and PE over MS.

Alternatively, MS might simply be a more difficult AAC system to teach because forming the signs requires more and varied physical movements than simply pointing to or handing a graphic to a partner (Study 1 - Chapter 5). Another possibility is that Sam and Nicky's failure to reach criterion for MS 
reflected the fact that they did not prefer to use it and were therefore less motivated to participate in the MS intervention sessions, once they started to make progress with the other options. This possibility suggests that preference, or lack of preference, for an AAC option may influence motivation to learn to use that option. If this explanation has validity, it would highlight the value of assessing preferences for different AAC options during the early stages of intervention, as was attempted in the present study. However, it is unclear how early such assessments might be implemented. Pre-baseline assessments could be configured, for example, but it is unclear if participants would require some level of exposure to each option before their choices would represent valid indicators of preference.

For one participant (Joe), it appeared that discrimination of graphic symbols was difficult. Specifically, Joe did not learn to discriminate the SNACK symbol from the PLAY symbol, and only reached criterion for SGD and PE when the distracter (PLAY) symbol was removed. As with Sam and Nicky's failure to reach criterion for MS, Joe's difficulty could reflect either a problem in his discrimination learning abilities or ineffective instructional procedures. In any event, these problems in teaching Sam, Nicky, and Joe suggest there may be some value in implementing a pre-intervention assessment of children's learning and behavioural characteristics (Light et al., 1998), such as determining the level of iconicity appropriate for an individual to acquire graphic symbol and MS understanding (Koul et al., 2001). From a research perspective it is important to ensure each AAC system is comparable in terms of cognitive demands so as to maintain functional equivalence in order to compare acquisition and preference between AAC systems (Schlosser, 2003c). It might also be important to ensure a match between the AAC system and skills being taught and the instructional strategies that are implemented to teach that system and those skills.

A limitation of the present study is that the PE system was not equivalent to the SGD and MS systems. While the SGD and MS options required only a one-step request, the former included two steps. That is, for the PE option, participants were required to not only place the I WANT, but also the corresponding SNACK or PLAY symbols, depending on whether they were requesting snacks or toys, onto the two locations provided on a separate card. 
This may have increased the response effort for the PE system, which in turn may have negatively influenced acquisition and preferences (Ringdahl et al., 2009; Winborn-Kemmerer et al., 2009). Although this did not appear to influence acquisition of the PE system in the present study, it may have diminished preference for that system. However, Sam did in fact demonstrate a preference for PE. Furthermore, due to the inherent differences in response topographies of SGD and PE versus MS, another potential limitation outlined in Study 1 (Chapter 5) was that a MS response could be produced during SGD and PE sessions, but not vice versa, possibly influencing preferences and rapidity of acquisition.

Joe, Saskia, and Nicky appeared to show a preference for using the SGD. While this could suggest that it was easier to use than either the PE or MS option, it is also possible that the SGD required somewhat more refined motor control, which might in fact make this a more difficult option to learn. That is, activating the speech-output function of the $\mathrm{iPod} 囚$-based SGD required a level of finesse (i.e., lightly touching or tapping the icon), which has been documented to be difficult for some adolescents with DD to master (Study 1 - Chapter 5; Kagohara et al., 2010; van der Meer, Kagohara, et al., 2011). Despite what could be a slightly more difficult system to activate, Joe, Saskia, and Nicky showed a preference for using the SGD. While it was suggested in Study 1 (Chapter 5) that some participants may prefer AAC options that are easier to use, others may prefer SGDs due to the dynamic display and speech-output features. Therefore, perhaps in addition to ease of use, it could be hypothesised that inherent features of some AAC options (e.g., speech-output) influence such preferences as suggested by Sigafoos et al. (2005). While the present results suggest children showed idiosyncratic preferences for the AAC options, future research would be needed to determine variables that might influence such preferences.

Joe and Sam did not come to make any consistent choices for one communication device over the others until they had reached criteria with each system. Saskia and Nicky, in contrast, appeared to show a preference (for the SGD) before they had learnt to use the communication option. These results suggest that preference for different AAC options may emerge at different times in the intervention process. In line with Study 1 (Chapter 5) Joe and Saskia showed better performance during follow-up with their preferred communication 
option. This finding suggests that preference may influence maintenance of newly acquired AAC-based requesting skills. Future research is needed to examine whether these findings might extend to interventions that focus on teaching more complex communication skills, such as asking and answering questions and commenting on the environment.

It did not appear that differing reinforcement histories accounted for the children's preferences for the different AAC options because they received the same number of sessions/reinforcements with each option during baseline and intervention. While Sam did later receive differential reinforcement schedules for the SGD and MS options in an effort to increase his performance with these two options, he was already showing a preference for PE prior to this procedural manipulation. However, it could be that the children's prior (pre-baseline) experiences may have influenced their preferences to some degree. Specifically, prior to this study, it appeared that while none of the children had any experience with SGDs, they reportedly had experience with one or more of the other AAC modes. Joe, Sam, and Saskia, for example, were reported to have had prior experience with MS and Joe and Nicky were reported to have had some experience with PE. Unfortunately, it is impossible to know if any of these prior experiences influenced their learning rates and choices during the AAC preference assessments that were conducted in this study. Future research could be improved by controlling for the potential bias that may arise when children enter a study with differing amounts and types of prior experiences with the to be compared AAC options. In practice, however, it may be difficult to determine the precise amount and nature of any such prior experiences given the often subjective and anecdotal nature of the information available to researchers about their prior AAC experiences. It is also perhaps inevitable that children with $D D$ who have limited or no speech will be exposed to one or more AAC modes and that such exposure could influence acquisition of, and preference for, different AAC modes. It is argued that even with such difficulties and uncertainties with respect to children's prior AAC experiences, it would still seem useful to assess their preference for different $A A C$ options so as to promote greater selfdetermination. 
In summary, the results of the present study extend the findings of Study 1 (Chapter 5) by comparing acquisition of, and preference for, three commonly used AAC modes (SGD, PE, and MS) among four children with DD. The results showed that two children learnt to use all three AAC modes, whereas the other two children learnt to use SGD and PE, but not MS. Preference checks suggested that three of the four children appeared to prefer using the SGD, whereas the other child showed a preference for using PE. Preference appeared to influence acquisition and maintenance, but more research is needed to confirm any such effects. 


\section{CHAPTER 7}

\section{STUDY 3}

\section{A Further Comparison of Manual Signing, Picture Exchange, and Speech- Generating Devices as Communication Modes for Children with Autism Spectrum Disorders ${ }^{6}$}

\section{Aims and Hypothesis}

Interesting questions arising from Studies 1 (Chapter 5) and 2 (Chapter 6) include (a) whether similar results would be found with additional children with ASD, (b) whether a child's relative preference for different AAC modes are present prior to intervention and remain stable over time after intervention, and (c) whether the findings of Studies 1 (Chapter 5) and 2 (Chapter 6) would have generality when teaching more specific requesting forms (e.g., "I want to play with the ball." "I want to play with the doll.") as compared to the more general requesting forms (e.g., "I want to play.") taught in the two previous studies. A final question is whether parents and general teaching staff could learn to effectively implement the intervention procedures successfully applied by the author in Studies 1 (Chapter 5) and 2 (Chapter 6).

The purpose of the present study was therefore to assess: (a) whether four new children with ASD diagnoses could be taught by their parents and general teaching staff to use specific requesting forms with three different AAC modes (i.e., SGD, PE, and MS); (b) whether these participants would demonstrate a preference for one communication modality over the others prior to and during intervention; (c) whether any such preferences would be stable over time; and (d) whether any such preferences would influence acquisition rates and subsequent performance during follow-up sessions. Based on the previous research and theoretical perspectives reviewed in Chapter 3 and from the findings of Studies 1 and 2 (Chapters $5 \& 6$ ), it was hypothesised that the four participants would learn to make specific requests at varying rates with the three communication modes, but that they would make the slowest progress with MS. It

\footnotetext{
${ }^{6}$ An article based on this study has been published in Research in Autism Spectrum Disorders: van der Meer, L., Sutherland, D., O'Reilly, M., Lancioni, G., \& Sigafoos, J. (2012). A further comparison of manual signing, picture exchange, and speech-generating devices as communication modes for children with autism spectrum disorders. Research in Autism Spectrum Disorders, 6, 1247-1257 (see Appendix G).
} 
was further hypothesised that most children would prefer using the SGD and that their relative preference for the three AAC modes would be stable over time. Finally, it was predicted that the children would show better maintenance of requesting with their most preferred $A A C$ mode.

\section{Method}

\section{Participants}

Four children who met the following inclusion criteria participated in this study: (a) diagnosis of ASD, (b) school-aged children of less than 18 years of age, (c) very limited or no communication skills as determined by an age equivalency of 2.5 years or less in the expressive communication sub-domain of the Vineland Adaptive Behavior Scales (Vineland-II; Sparrow et al., 2005), (d) no auditory or visual impairments that would interfere with the use of $A A C$, and (e) sufficient motor skills to operate each of the three AAC communication modes, as determined by an age equivalency of 1.0 year or more on the fine motor skills sub-domain of the Vineland-II.

Jason. Jason was a 4-year-old male diagnosed with autism and global developmental delay. On the communication sub-domain of the Vineland-II (Sparrow et al., 2005), Jason received age equivalencies of 2:5 (years:months) for receptive communication, 1:8 for expressive communication, and 3:1 for written communication. His age equivalency on the fine motor skills sub-domain of the Vineland-Il was 2:11. Jason was reportedly able to use a few single words to make requests and comment on his environment, although his speech was largely unintelligible. He also appeared to attempt to communicate his wants and needs by taking people's hands and leading them. Jason had been informally introduced to $P E$ in the past, but was not receiving any such training throughout the period of this study. This prior PE experience was independent of, and unrelated to, the the present intervention. He did not have any prior experience with SGD or MS to request preferred objects.

Jack. Jack was a 4-year-old male diagnosed with autism. His age equivalencies were 1:3, 0:8, and 1:10 on the receptive, expressive, and written communication sub-domains of the Vineland-II (Sparrow et al., 2005). Jack received an age equivalency of 2:0 on the Vineland-II for fine motor skills. He did not have any spoken language, but had prior experience with PE that was 
independent of, and unrelated to, the the present intervention. As a result of this prior experience, he was reportedly able to discriminate among 20 PE symbols that he used to request food items. Otherwise his communication attempts involved leading a person's hand to desired objects. He had no experience with SGD or MS for requesting preferred items.

Ian. Ian was a 10-year-old male diagnosed with autism, moderate intellectual disability, developmental co-ordination disorder, and epilepsy. $\mathrm{He}$ received age equivalencies of 2:2, 1:4, and 3:10 on the receptive, expressive, and written communication sub-domains of the Vineland-II (Sparrow et al., 2005). He received an age equivalency of 2:1 on the fine motors skills sub-domain of the Vineland-II. lan had no formal means of expressing himself, but attempted to create his own signs for words, that were difficult to decipher. lan also tried to sound out some words, but this was unintelligible. He would often take people's hands to direct them to what he wanted and frequently grabbed people in an apparent attempt to get their attention. lan did not have any prior training with $S G D, P E$, or MS to request preferred items. lan's mother indicated that he engaged in obsessive behaviour (e.g., fixation on certain toys) and had difficulty with changes to routine.

Hannah. Hannah was an 11-year-old female diagnosed with autism, severe global developmental delay, and intellectual disability. On the Vineland-II (Sparrow et al., 2005), she received age equivalencies of 1:3 for receptive communication, 0:9 for expressive communication, and 2:5 for written communication. She received an age equivalency of 2:11 on the fine motor skills sub-domain of the Vineland-II. Hannah appeared to communicate her wants and needs by taking people's hands and leading them to objects. She had received training with PE and had been exposed to several gestures, as well as a BIGmack ${ }^{\circledR}$ SGD on several occasions. These prior experiences were independent of, and unrelated to, the present study. She appeared to make vocalisations to communicate pleasure and distress.

\section{Setting, Intervention Context, and Trainer Instruction}

Jason, Jack, and lan received intervention in the dining room of their family home. Hannah received intervention sessions in a special education classroom that was part of a public primary school. For Jason, Jack, and lan the 
procedures associated with this study were conducted at a table and implemented in a one-to-one format, consisting of the trainer (mother) and child. Hannah's intervention was implemented at a table in the special education classroom in a one-to-one context consisting of the trainer (teaching assistant) and Hannah.

Jack, Jason, and lan's mothers were taught how to implement the procedures by the author. This parent instruction involved (a) explaining the general aims, goals, and procedures of the study, (b) providing parents with stepby-step written instructions for each phase of the study and explaining these steps prior to each phase of the study, (c) modeling implementation of the steps during the first trials at the beginning of each new phase and then having the parent implement the remaining trials for that session, and (d) providing feedback at least once per week throughout each phase of the study. The same strategies were used to teach the teaching assistant how to implement the procedures with Hannah.

\section{Preferred Stimuli}

Snacks and/or toys that the participants seemed to prefer, and which would be appropriate for the participants to request during the snack/leisure activity, were identified using a two-stage stimulus preference assessment (Green et al., 2008). Stage 1 involved an indirect assessment in which parents/teachers were asked to list snacks and toys that the participants appeared to enjoy and would be appropriate for the intervention. The five to six most preferred stimuli were then selected for a direct stimulus assessment, involving the simultaneous presentation of multiple items, without replacement (DeLeon \& Iwata, 1996; Duker et al., 2004). Each participant was presented with an array of five to six items from Stage 1 (random placement) and allowed to select one. A session consisted of repeating such offers five or six times depending on whether five or six items had been placed on the tray. Items were not replaced once they had been selected. Toy and food items were assessed separately over six sessions (i.e, six sessions with toys and six sessions with foods). The most preferred foods and/or toys were identified by calculating a rank order of the percentage of times that an item was selected using the formula: Number of Selections/Number of Offers $\times 100 \%$. 
Preferred stimuli selected for Jason were marshmallows (75\%), balloons and bubbles (both 67\%), and chips (35\%). Preferred stimuli for Jack were wafer biscuits (55\%), K'NEX® building blocks (similar to Lego $® ; 43 \%$ ), waterplay toy (40\%), and caramel lollies (38\%). Preferred stimuli for lan were music box (100\%), puzzles $(40 \%)$, hide and seek game $(30 \%)$, and bouncy balls $(29 \%)$. Preferred stimuli for Hannah were M\&M® candy (50\%), bubbles (46\%), chocolate (43\%), and music box (32\%).

\section{SGD}

Jason, Jack, and lan were taught to request preferred stimuli using an Apple iPod Touch $®$ with Proloquo2Go ${ }^{\mathrm{TM}}$ software (Sennott \& Bowker, 2009). The iPod Touch $®$ was placed inside an iMainGo2 $\AA$ speaker case to increase sound amplification (Figure 7.1).

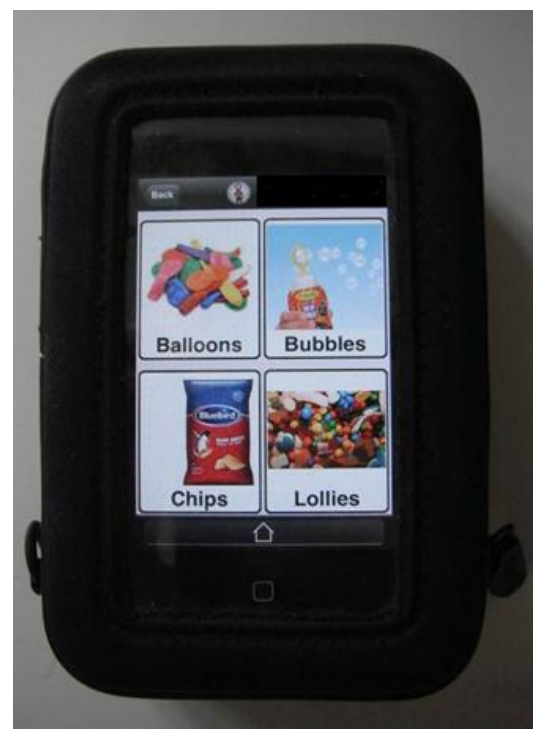

Figure 7.1. Photograph of the iPod Touch $^{\circledR}$ Inside the iMainGo ${ }^{\circledR} 2$ Speaker Case with the Visual Display of the Graphic Symbols of each Preferred Item Targeted for Requesting for Study 3.

Hannah, and later Jack, were taught to use an Apple iPad® with Proloquo2Go ${ }^{\mathrm{TM}}$ software (Figure 7.2). The iPod Touch $®$ and $\mathrm{iPad} \circledast$ were configured to show a single page containing four graphic symbols $(2.5 \times 2.5 \mathrm{~cm}$ for the $\mathrm{iPod} \otimes$ and $9.5 \times 6 \mathrm{~cm}$ for the $\mathrm{iPad} \AA)$, representing requests for specific snacks and toys (e.g., CHOCOLATE, BALL). The graphic symbols were photos of the items uploaded into the Proloquo2 $\mathrm{Go}^{\mathrm{TM}}$ software package. Touching each 
symbol activated corresponding synthetic speech-output (e.g., "I would like some chocolate." and "I would like to play with my ball.").

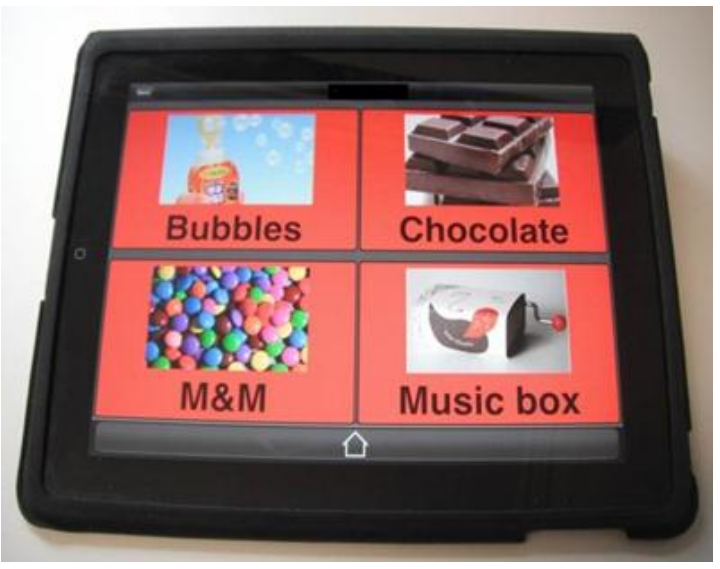

Figure 7.2. Photo of the $\mathrm{iPad}^{\circledR}$ with the Visual Display of the Graphic Symbols of each Preferred Item Targeted for Requesting for Study 3.

\section{PE}

The PE system consisted of four laminated photos $(7 \times 7 \mathrm{~cm})$ of the participant's most preferred items, with the corresponding printed word written below the photo. Photos were affixed with Velcro ${ }^{\mathrm{TM}}$ to a laminated card $(22 \times 22$ $\mathrm{cm})$. The pictures were randomly allocated to the four locations of the card (Figure 7.3).

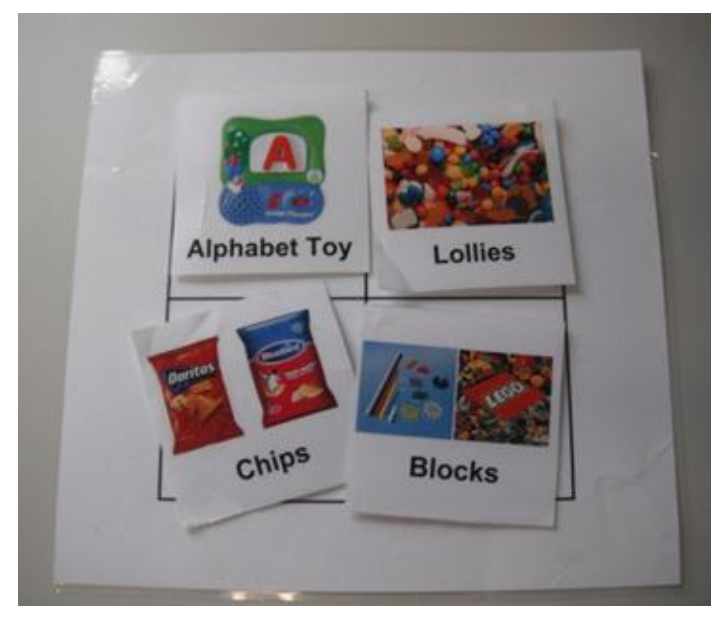

Figure 7.3. Photograph of the PE Communication Option with Graphic Symbols of each Preferred Item Targeted for Requesting for Study 3. 


\section{MS}

Participants were also taught to request preferred stimuli using signs from the Makaton Sign Language System (Makaton New Zealand/Aotearoa, 1998-99). Manual signing was represented by a laminated picture $(22 \times 22 \mathrm{~cm})$ with four line drawings $(8 \times 8 \mathrm{~cm})$ of the hand formations needed to make the sign for each of the children's preferred stimuli targeted for intervention (Figure 7.4).

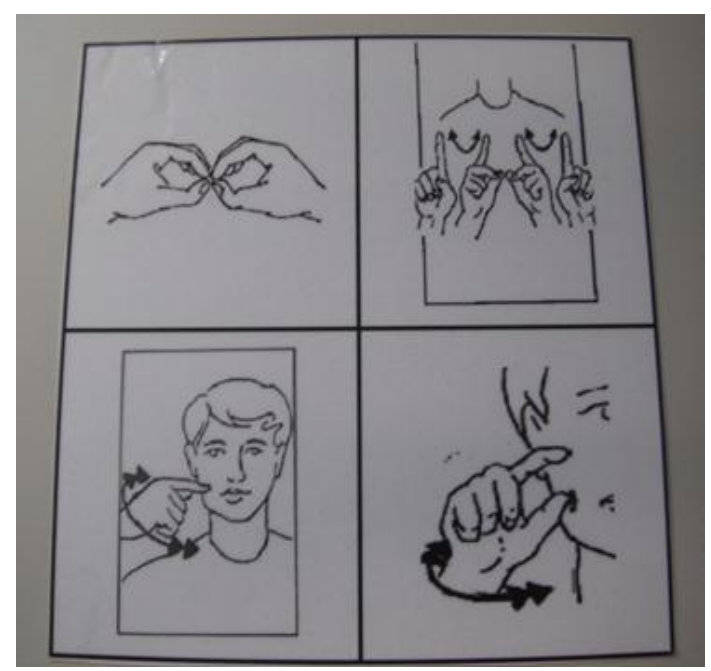

Figure 7.4. Photograph of the Makaton Signs of each Preferred Item Targeted for Requesting to Represent the MS Communication Option for Study 3.

\section{Response Definitions and Measurement}

For SGD use, correct responding was defined as independently (without a gestural or verbal prompt) touching the symbol on the screen of the SGD to activate the corresponding speech output in exchange for the item offered by the trainer. For PE, participants were required to independently (without gestural or verbal prompt) remove the corresponding picture from the PE card and hand it over to the trainer in exchange for the item being offered. Manual signing was defined as independent (without a gestural or verbal prompt) performance of the hand gestures to produce correct signs in exchange for the item offered by the trainer. Independent approximations of the sign (e.g., touching finger on side of lip for lolly, instead of specifically moving finger from cheek to side of lip) were counted as correct. The frequency of correct responding was calculated for each session. Sessions consisted of 12 trials (i.e., each of the four preferred items was 
offered three times). The order of offering items was counterbalanced across sessions.

\section{Experimental Design}

An alternating treatments design was used to compare intervention performance across the SGD, PE, and MS options (Barlow \& Hayes, 1979; Barrett \& Sisson, 1987). The study phases (i.e., baseline, intervention, preference assessments, post-intervention, and follow-up) were implemented according to a non-concurrent multiple-baseline across participants design (Watson \& Workman, 1981). The participants received differing lengths of baseline, but started their baselines in a staggered fashion due to their being recruited into the study at different points in time. Training was first provided to Jason, then Jack, then lan, and finally Hannah.

\section{Session Schedule}

Requesting sessions were conducted three to five days per week. The AAC option available (i.e., SGD, PE, or MS) was counterbalanced across sessions to prevent order effects (Kennedy, 2005). For all sessions, the participant and trainer were seated next to each other at a table. One or two additional observers/data collectors were seated nearby on some occasions to collect inter-observer agreement data and conduct procedural integrity checks.

When a participant reached criterion for one AAC device (i.e., 80\% correct requesting across three consecutive sessions), teaching for that system was put on hold while it continued with the other communication systems, if necessary, until criterion was reached on the other systems. One maintenance session with the acquired $A A C$ system was conducted after every third teaching session with the other (yet to be acquired) AAC systems.

\section{Procedures}

Participants were taught to request specific snacks or toys. To ensure symbol discrimination, participants had to select the graphic symbol from the SGD or PE board or produce the manual sign corresponding to the item being offered by the trainer. If a participant requested a different item to the one being offered, the trainer responded by saying: We are requesting (name of item) and pointed to the correct symbol on the SGD/PE board or modeled the correct sign. It was considered more natural to provide them with some feedback when they 
activated these (non-target) symbols or made the non-target sign, but counterproductive to discrimination training to consequate these non-target responses with access to preferred items. Similarly, producing MS to request items during SGD or PE sessions and vice versa was ignored so as to bring the use of each device under stimulus control.

Pre-baseline AAC preference assessments. These assessments were undertaken to familiarise participants with each of the three communication modes (SGD, PE, and MS) and to determine if participants showed a preference for one mode over the other two before learning to use them. During these sessions, the SGD, PE, and MS options were placed on the table in randomly determined locations. The trainer briefly explained and demonstrated each communication mode (e.g., This is a PE board. You use it like this.). The trainer then asked the participant: Which communication option would you like to use? Participants were allowed $10 \mathrm{~s}$ to select one of the communication options. Selecting was defined as touching, holding, and/or manipulating the device. If the child did not select one of the communication options within $10 \mathrm{~s}$, the next trial was initiated by again asking the participant: Which communication option would you like to use? Communication options were not replaced once selected. Instead, the remaining communication options were then offered. This procedure was repeated across six sessions (three offers per session) and the percentage of times that each device was selected was calculated using the formula: Number of Selections/Number of Offers x $100 \%$. Pre-baseline preference assessments were not undertaken with Hannah due to an oversight.

Baseline. During this phase, a tray containing one of the participants' four preferred items was placed on the table in view, but out of reach. The SGD, PE, and MS communication options were randomly placed on the table. Each session began with the trainer asking the participant to Let me know if you want this, while holding the tray with the item being offered. After $10 \mathrm{~s}$, the trainer moved the tray within reach and allowed the participant to take the item. This was repeated 12 times (trials) per session, with each of the four items offered three times in a counterbalanced order. This 10-s fixed-time schedule of reinforcement was provided to ensure continued motivation to participate in sessions. Participants were given approximately $20 \mathrm{~s}$ to consume/play with the item being 
offered. Responses to the SGD, PE, and MS were recorded, but had no programmed consequences.

Intervention. This phase was conducted in a discrete-trial format until participants reached criterion (i.e., $80 \%$ correct requesting across three consecutive sessions for each AAC mode) or, failing that, until they had received at least 10 sessions of intervention with each AAC mode. Each trial consisted of the trainer placing a tray with one of the participant's preferred items on the table, showing the item to the participant, but keeping it out of reach, and saying, Let me know if you want this. Training involved a 10-s time delay between the verbal cue (i.e., Let me know if you want this.) and the use of graduated guidance to prompt a correct request. Graduated guidance involved using the least amount of physical guidance necessary to prompt the child to make a request, with a simultaneous explanation of how to perform the action (e.g., Press the ball icon to ask to play with a ball. for SGD, or Hand over the picture of the ball. for PE, or Move your hands in the formation of a ball to make the sign for ball. for MS). Immediately after a correct SGD, PE, or MS request, the trainer moved the tray containing that item within reach of the participant with simultaneous social reinforcement (e.g., Good asking.). The participant was allowed approximately 20 $s$ to consume/play with that item. After this, the next trial was initiated. Twelve such trials (three trials per item) were conducted per session with the order of the items being offered counterbalanced to avoid order effects.

Procedural modifications. Because Jack made little progress with the SGD and MS options during the initial intervention sessions, his teaching procedures were modified to include 10 massed-practice trials before each SGD and MS session. This involved implementing 10 consecutive graduated guidance trials with no time delay and no reinforcement. The aim was to provide Jack with additional practice in making the target responses. With this modification, Jack began to initiate correct requests with the SGD, but appeared to struggle to press the icons with the appropriate degree of finesse to activate the voice output. It was therefore decided to switch to an $\mathrm{Pad} \circledast$ because we thought that the larger size of icons and increased sensitivity of the screen on the $\mathrm{Pad} \otimes$ would make it easier for Jack to activate the SGD. Although Jack made some progress with these modifications to the intervention process, he also rather quickly appeared 
to lose interest in the preferred stimuli that he was being taught to request. Therefore another stimulus preference assessment was implemented. New preferred stimuli were then chosen for intervention consisting of lollies $(60 \%)$, blocks (46\%), an alphabet toy (40\%), and chips (24\%).

AAC preference assessments. These assessments were undertaken after each baseline, intervention, and follow-up session to determine if participants would show a preference for using one of the three AAC systems. They were identical to pre-baseline device preference assessments, except when the participant selected a communication mode, the trainer then initiated one requesting opportunity with the chosen mode before reverting back to another baseline, intervention, or follow-up session. If the participant did not choose an option within $10 \mathrm{~s}$, that AAC preference assessment trial was terminated and training continued with the AAC option that was scheduled for use in that session.

Post-intervention. Once the participant reached criterion for each AAC device, post-intervention preference assessments were introduced. These were identical to the previously described AAC preference assessments, except that once an AAC option had been chosen, the participant continued to request preferred items using the chosen communication method for the entire session.

Follow-up. Nine follow-up sessions (three for each communication mode) were conducted after Session 24 for lan and after Session 45 for Jason (see Figure 7.6). These occurred three weeks after their last post-intervention session. lan also received a second set of [long-term] follow-up sessions beginning eight months after his previous follow-up sessions (Session 33 of Figure 7.6). Hannah received follow-up after Session 68 (Figure 7.6), eight weeks after her last postintervention session. Because of a change of schools in the interim, Hannah's follow-up sessions were conducted by the author, not by the teaching assistant. During the interval, participants did not use any of the communication modes to request their preferred items. Jack did not receive follow-up because he did not reach criterion for any of the communication modes. Procedures for follow-up were identical to the intervention phase, except no prompting occurred and participants only received access to preferred items contingent on correct requesting. Also an AAC preference assessment (as described in $A A C$ preference assessments) was implemented after each session. 


\section{Inter-Observer Agreement}

Trainers collected data on the frequency of correct requesting and on which communication mode was selected during AAC preference assessments. To assess the reliability of the trainers' data collection, an independent observer also collected data on the frequency of requesting and communication mode chosen. For each session, percentages of agreement between the independent observer and the trainer were calculated using the formula:

Agreements/(Agreements + Disagreements) $\times 100$. These agreement checks occurred on $32 \%$ of all sessions and ranged from 95 to $99.7 \%$ with a mean of $97.8 \%$.

\section{Procedural Integrity}

To assess procedural integrity, the independent observer had a checklist of the procedural steps and recorded whether the trainer had correctly implemented each procedural step in its proper sequence. Procedural integrity was assessed on $32 \%$ of all sessions and ranged from 98.8 to $99.9 \%$ correct implementation with a mean of $99.1 \%$. A second independent observer collected inter-observer agreement data on $11 \%$ of these integrity checks with $100 \%$ agreement.

\section{Results}

Table 7.1 provides a summary of the pre-baseline preference assessments. Figure 7.5 provides a summary of the results from the AAC preference assessments conducted during baseline, intervention, and subsequent phases. Figure 7.6 shows the percentage of correct requests during each session for each of the three AAC modes. In baseline (Figure 7.6), none of the participants ever used PE or MS to make the targeted requests. lan and Hannah made several correct SGD-based requests during baseline. However, a stable and low baseline was evident for all children prior to introducing intervention. 
Table 7.1. Percentage of Selection/Times Offered for SGD, PE, and MS During the Pre-Baseline AAC Preference Assessments.

\begin{tabular}{lllc}
\hline Child & SGD & PE & MS \\
\hline Jason & 100 & 20 & 6 \\
Jack & 55 & 45 & 29 \\
lan & 86 & 50 & 29 \\
Hannah & - & - & - \\
\hline
\end{tabular}

NAP was used to calculate effect sizes (Parker \& Vannest, 2009). Table 7.2 shows the resulting percentages of NAP. Effect sizes for each participant for treatment effects from baseline to intervention across communication options (SGD, PE, and MS) were calculated. "Large" ( $\geq 80 \%$ ) effect sizes (Cohen, 1988; Parker \& Vannest, 2009) were found for three participants (Jason, lan, and Hannah) for intervention effects for SGD and PE. Only two of the participants (lan and Hannah) obtained high intervention effects for MS. Greater effects across participants were achieved for PE $(M=98.25 \%)$, then SGD $(M=90.75 \%)$, and followed by MS $(M=82.5 \%)$. As in Studies 1 (Chapter 5 ) and 2 (Chapter 6), higher effect sizes for $P E$ and SGD reflected participant's preferences for using PE and SGD. Descriptions of each participant's acquisition and preference are provided below.

Jason

When intervention was introduced, Jason reached the acquisition criterion for the SGD option on his third SGD training session (Figure 7.6). Although he did not reach criterion for $\mathrm{PE}$ and $\mathrm{MS}$, Jason achieved above $80 \%$ correct requesting in three of his PE ( $M=86 \%$ range $=33$ to $100 \%)$ and two of his $\mathrm{MS}(M=51 \%$ range $=0$ to $100 \%$ ) intervention sessions. During the post-intervention phase, Jason chose to use the SGD $100 \%$ of the time. Once chosen, he then used the SGD with at least $80 \%$ proficiency. During follow-up, Jason maintained correct SGD- and PE-based requests at high levels (63-100\%), but his performance dropped to between 36 and $67 \%$ correct for MS. He chose the SGD first on 100\% of opportunities during the pre-baseline device preference assessments (Table 7.1). Overall, during baseline, intervention, post-intervention, and follow-up 
phases, Jason received a total of 45 opportunities to choose between the SGD, PE, and MS options (Figure 7.5) and he chose the SGD most frequently (69\%).

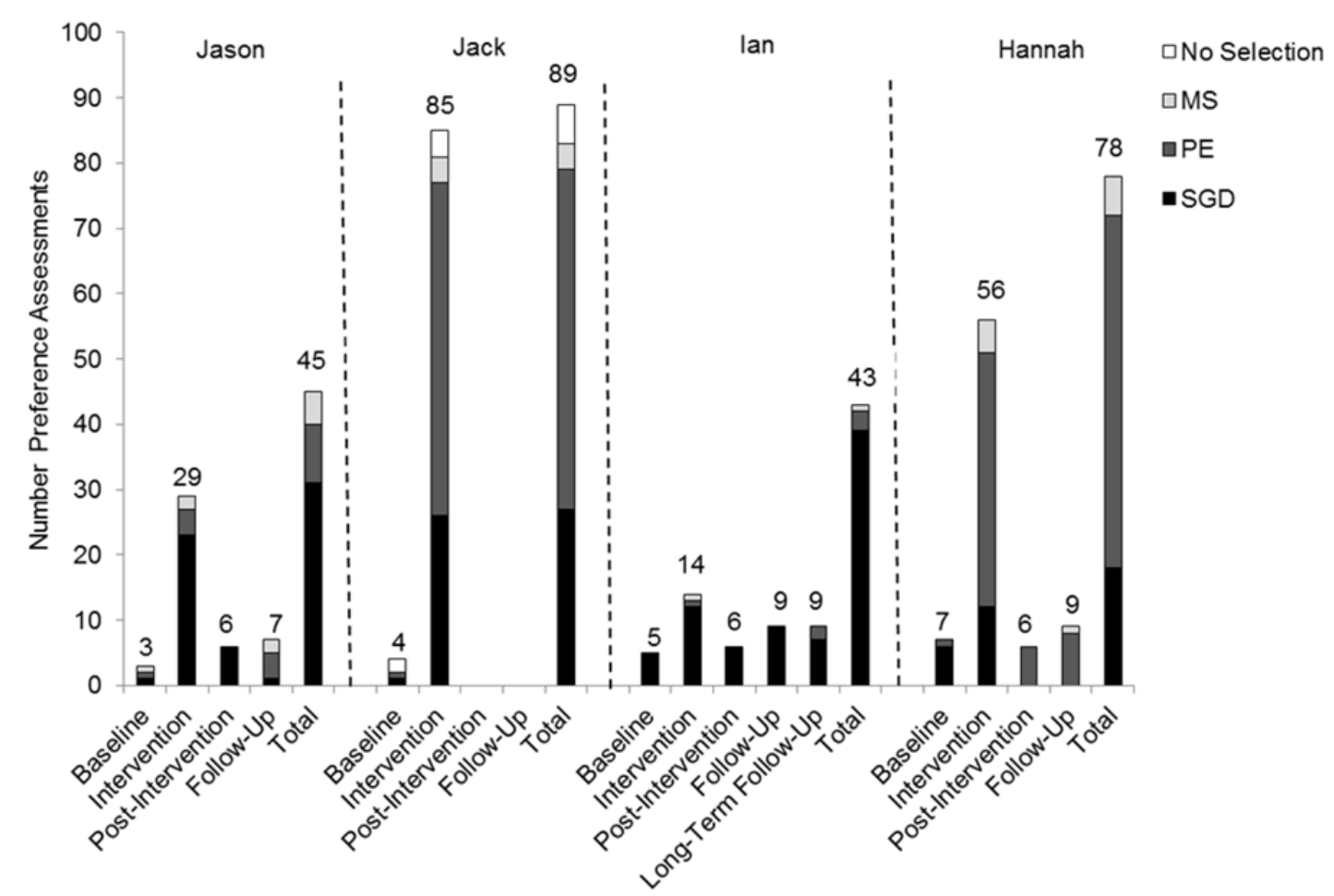

Figure 7.5. Results from the AAC Preference Assessments Depicting the Number of Times each Communication Option (SGD, PE, and MS) was Chosen Across Phases and Participants.

\section{Jack}

Although Jack did not reach criterion with PE, he did achieve over $80 \%$ correct requesting on a number of occasions. His performance with the PE mode averaged 52\% (range $=8-92 \%$ ) correct requesting. Similarly, even with modifications to the intervention procedures, he did not reach criterion with the iPod Touch $\Omega(M=5 \%$ range $=0-22 \%)$. When the $\mathrm{Pad} \otimes$ was introduced, his performance increased to an average of $35 \%$ (range $=17-67 \%$ ) correct requesting. Even with additional procedural modifications, Jack made little progress with MS and did not achieve criterion within the timeframe of this study. Therefore he did not progress to the post-intervention or follow-up phases. Results from the pre-baseline preference assessment indicated that Jack chose each communication system comparably often (Table 7.1). During baseline and 
intervention, Jack received 89 AAC preference assessments (Figure 7.5) and he chose PE most frequently (58\%), followed by the SGD (30\%).

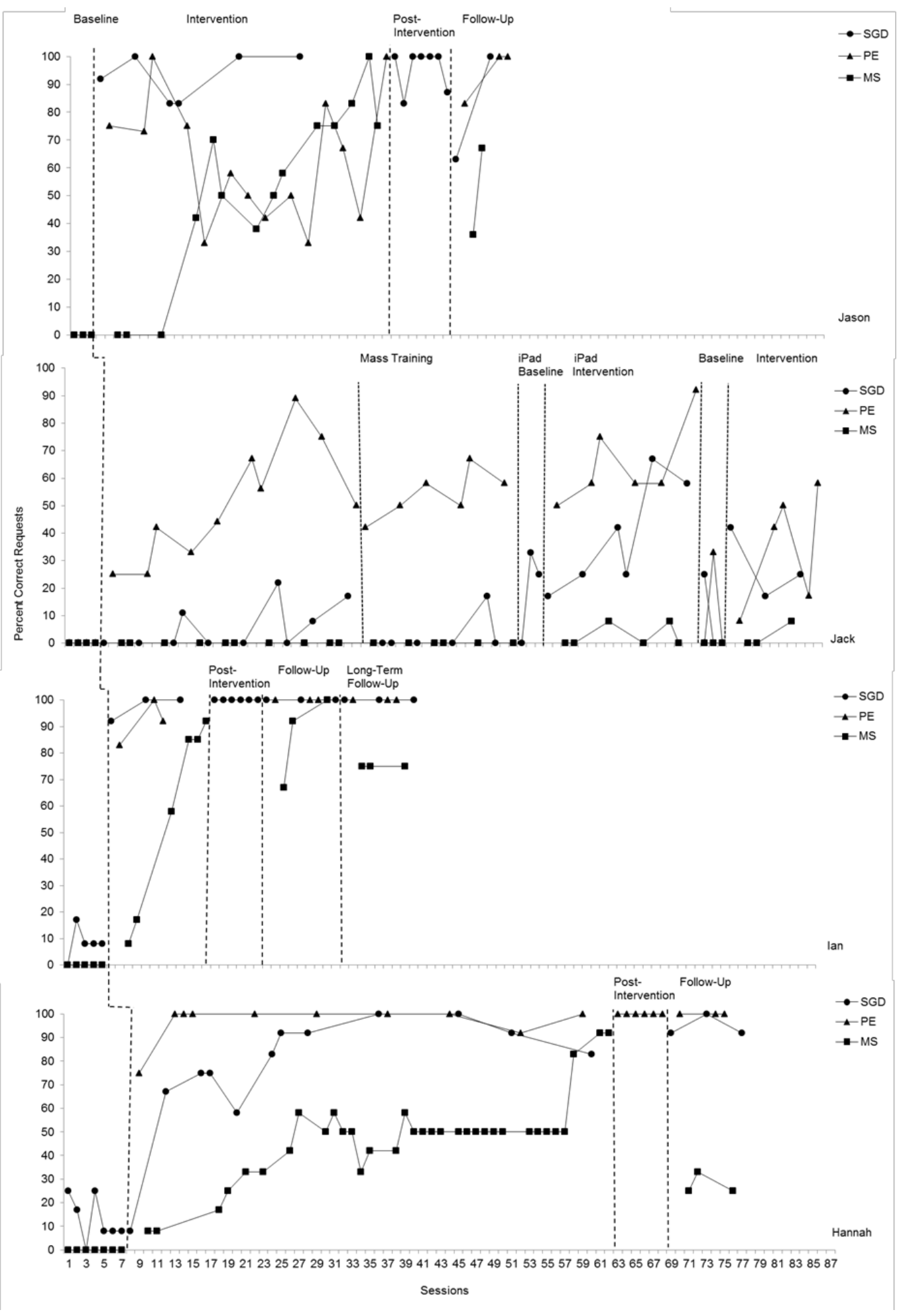

Figure 7.6. Percentage of Correct Requests with SGD, PE, and MS across Sessions for each Participant. 
Table 7.2. Baseline to Intervention NAP Effect Sizes for SGD, PE, and MS for each Participant.

\begin{tabular}{llll}
\hline Participant & SGD & PE & MS \\
\hline Jason & $100 \%$ & $100 \%$ & $79 \%$ \\
Jack & $74 \%$ & $93 \%$ & $51 \%$ \\
lan & $100 \%$ & $100 \%$ & $100 \%$ \\
Hannah & $89 \%$ & $100 \%$ & $100 \%$ \\
\hline
\end{tabular}

NAP is rescaled to a $0-100 \%$ scale

Ian

Ian achieved criterion for SGD- and PE-based requesting on his third SGD and third PE intervention sessions (Figure 7.6). He achieved criterion for MSbased requesting on his sixth MS intervention session (Figure 7.6). During the post-intervention phase, lan always chose to use the SGD and then used it with $100 \%$ proficiency. During the first follow-up, his performance maintained at $100 \%$ correct for the SGD and PE, but decreased with the MS option. During the second, long-term follow-up (after eight months), his performance with the SGD and PE option was at $100 \%$, whereas it was at $75 \%$ for the MS option. lan chose the SGD most frequently (86\%) during the pre-baseline AAC preference assessments (Table 7.1). During baseline, intervention, post-intervention, and follow-up phases, lan received 43 AAC preference assessments (Figure 7.5) during which he chose the SGD the majority of the time (91\%).

\section{Hannah}

Hannah achieved acquisition criterion for PE- and SGD-based requests on her fourth and eighth respective intervention sessions (Figure 7.6). She showed steady increases in the percentage of correct requests using MS, and finally reached criterion on her $34^{\text {th }} \mathrm{MS}$ intervention session (Figure 7.6). During the post-intervention phase, Hannah always chose to use PE and then used it with $100 \%$ proficiency. During follow-up, SGD and PE use maintained at high levels (92-100\%), while MS dropped to 25 to $33 \%$ correct requesting. Hannah did not receive pre-baseline AAC preference assessments. During baseline she chose the SGD $68 \%$ of the time, but overall across the total of 78 AAC preference assessments conducted during the study (Figure 7.5), she chose PE most frequently $(69 \%)$. 


\section{Discussion}

The first aim of this study was to determine whether children with ASD could learn to request preferred items using one of three different AAC systems. It was predicted that these children would learn to request preferred items using one of the three different AAC systems. The data was consistent with this prediction. All four participants learnt to make specific requests for preferred items using at least one of the three AAC modes taught. Specifically, two participants (lan and Hannah) reached criterion with all three communication modes, while one participant reached acquisition criterion with the SGD and one (Jack) learnt to use PE with moderate proficiency. These findings are consistent with previous studies reporting that children with ASD can learn to use SGDs, $\mathrm{PE}$, and MS to make requests for preferred items (Flippin et al., 2010; van der Meer \& Rispoli, 2010; Wendt, 2009). Furthermore, Studies 1 (Chapter 5) and 2 (Chapter 6) showed that eight children with DD learnt to use SGD, PE, and MS to make general requests for snacks or toys. The present results extend these studies by demonstrating that four new children with ASD could concurrently learn to use SGD, PE, and MS to make specific requests.

The present results also extend previous research by showing that parents (i.e., Jason, Jack, and lan's mothers) and a teaching assistant (for Hannah) could effectively apply the intervention procedures. Given that parents and teaching staff are arguably the children's more important communicative partners, this extension increases the applied relevance of the present findings, relative to Studies 1 (Chapter 5) and 2 (Chapter 6), in which research staff implemented the procedures. The strategies for training these parents and the teaching assistant appeared to have been effective as evidenced by (a) the generally positive results with respect to acquisition of MS-, PE-, and SGD-based requesting, and (b) the high degree of procedural integrity. The fact that parents and the teaching assistant learnt to effectively implement the procedures with relatively little training is not surprising given that the strategies utilised for doing so were consistent with best practices for training paraprofessionals to implement similar types of behaviourally-oriented teaching programs (Reid, O'Kane, \& Macurik, 2011). 
The findings also demonstrated variability with which children with ASD learnt the three AAC modes in line with the findings of Studies 1 (Chapter 5) and 2 (Chapter 6). For example, one participant (Jack) did not learn to use MS and the remaining participants mastered MS at a slower rate than the other communication modes. Study 2 (Chapter 6 ) provides three possible reasons for this finding: (a) it may be more difficult to learn MS because it requires recall memory whereas graphic symbols (used for SGD and PE) could be less demanding on children's working memory - only needing recognition memory (lacono \& Duncum, 1995; lacono et al., 1993), (b) MS may be a more difficult AAC system to teach because forming signs requires more physical motions than pointing to or handing over a graphic symbol to a communication partner (SGD and $\mathrm{PE}$ ), or (c) MS was the least preferred option, which may have reduced motivation to use it.

The second and third aims of this study were to examine whether children would indicate a preference for using one AAC system over the others during the early stages of acquisition and whether any such preferences would be stable over time. The initial choice-making patterns demonstrated by three of the participants (Jason, lan, and Hannah) suggested a preference for using the SGD, while the remaining participant (Jack) chose each AAC system comparably often. Results suggested that for three participants (Jason, Jack, and Hannah), the prebaseline and/or baseline choices for the different AAC systems were more variable, with preferences changing throughout later phases of the study as they learnt to use each AAC mode. Therefore it could be hypothesised that some children might indicate an immediate preference based (perhaps) on some inherent features of the available AAC options (e.g., voice-output or dynamic display of SGDs), but that as they gain proficiency in using each AAC mode their preferences may change to reflect other features, such as ease of use. This could also explain lower rates for mastery and preference of MS in that the MS option might have less instant appeal. This is turn could mean that some children were less motivated to learn to use the MS option. Further, the findings indicated that for some participants, their preferences for the three AAC modes only seemed to be stable once they achieved acquisition with each AAC mode. One implication of this finding is that preferences might be reliably assessed, and 
remain stable only after children have learned to use each communication mode to some [high] level of proficiency.

The final aim was to ascertain whether any demonstrated AAC system preferences would influence acquisition and maintenance of newly acquired requesting skills. Three participants (Jason, lan, and Hannah) learnt to use and/or achieved criterion with each communication system. In addition, the follow-up data suggested that maintenance was higher when these three children were using their most preferred AAC mode. Interestingly, although Jason demonstrated an overall preference for the SGD prior to follow-up, his preference shifted to PE during the follow-up phase and he also performed at a higher rate with $P E$, than with the SGD, during the follow-up phase. This suggests that for some children, their preferences for different AAC modes may change over time and influence performance. The implication of this is that there might be value in periodically reassessing children's preferences after intervention, even when they showed a preference for a particular AAC mode during acquisition and immediately after acquisition.

The participants in the study reliably discriminated between symbols used on the PE and SGD modes. This contrasts with prior research that described the difficulties children with ASD have in learning to use aided communication systems (SGD and PE) due to the symbol discrimination (matching-to-sample) and scanning repertoire necessary to differentiate between and select symbols (Cannella-Malone et al., 2009; Koul et al., 2001; Sundberg \& Sundberg, 1990; Wraikat et al., 1991). The use of photos of specific preferred stimuli in the present study may explain why participants relatively quickly learnt to discriminate and select the correct symbol corresponding to the item being offered. However, further research is required to validate the hypothesis that photos of specific items could facilitate the acquisition of SGD- and PE-based requesting (De Paul \& Yoder, 1986; Johnston \& Cosbey, 2012).

Activation of the iPod Touch $®$ appeared to be problematic for Jack in that he appeared to have difficulty selecting screen icons with sufficient finesse to activate the speech output. This problem has been noted in previous studies (Cannella-Malone et al., 2009; Kagohara et al., 2010) as well as Studies 1 and 2 of the present thesis. In an attempt to rectify this problem, use of an $\mathrm{Pad} \otimes$ was 
introduced. As anticipated, the larger display appeared to be helpful and Jack's percentage of correct SGD-based requests increased. Massed-practice trials were also introduced in an attempt to teach Jack the MS-based request. However, he only made minimal progress in learning to make the sign for LOLLY. To some extent, his relatively limited progress with all three communication modes appeared to be a motivational issue in that he did not consistently select and then play with or eat the presumed preferred stimuli. Even after a second reinforcer assessment, and subsequent introduction of new preferred stimuli Jack seemed to quickly lose interest in the items he was being taught to request. The implication here is that progress in teaching AAC-based requesting to some children, regardless of mode, is likely to be compromised in the absence of powerful reinforcers.

While the overall findings were generally positive and consistent with previous research, several limitations must be considered when interpreting the present data set. First, only four children participated in this study and therefore care should be taken when considering the implications of the findings with other children. However, when these results are combined with Studies 1 (Chapter 5) and 2 (Chapter 6 ) there are now a total of 12 children with DD who have shown largely similar results. Still, further research involving increased numbers of children is needed to extend the generality of the findings. Second, no data were obtained on generalisation of the requesting skills taught during the study to other preferred items, settings, or communicative partners. Research investigating generalisation across the three AAC modes would be an obvious future direction. In addition, the present study focused only on teaching requesting (mands). It would be instructive to consider the use of MS, PE, and SGDs when teaching other communicative functions, such as tacting and intraverbal behaviour (Skinner, 1957). Third, further research should examine factors that might influence a child's preference for using one AAC system over others. This is particularly relevant research considering the present findings suggest that such preferences can influence performance during intervention and follow-up, a finding that was also noted by Ringdahl et al. (2009).

In summary, the present results support previous findings that children with ASD and other DD can be taught to use three common AAC systems to 
make requests and will also often show a preference for using one AAC system over others. The results extend the previous studies (Chapter 5 - Study 1 \& Chapter 6 - Study 2) by showing that while AAC mode preferences may be present prior to intervention, they can often change during and after the intervention phase. In addition, the present results provide further evidence to suggest that acquisition of AAC-based requesting is faster and maintenance is better when children use their more preferred AAC mode. The results thus provide more evidence to support the value of assessing children's preferences for different $A A C$ modes when designing and implementing communication interventions. 


\section{CHAPTER 8}

\section{GENERAL DISCUSSION}

\section{Main Findings}

Across the three studies a total of 12 children ( 9 boys, 3 girls, aged 4 to 13 years) received intervention. The results of this intervention showed that each of the participants learnt to request preferred items with at least one of the AAC systems (SGD, PE, and MS) targeted for intervention to some (high) level of proficiency. In addition all 12 participants appeared to have demonstrated a preference for using one communication option over the others. The results suggest that the intervention procedures were effective for teaching the children to use at least one AAC mode to request preferred items and that the AAC choice-making protocol was an effective approach for assessing children's preferences across the three AAC options.

Specifically, in Study 1, which involved a comparison of SGD and MS, three (David, Tom, and Eli) of the four participants reached criterion on the use of both of these communication options. However, the other participant (Zac) did not learn to use MS within the timeframe of the intervention. Furthermore, three (Tom, Zac, and Eli) of the four participants demonstrated faster acquisition of, and a preference for using, the SGD, while the other participant (David) appeared to have a slight preference for using MS. David also reached acquisition criterion for MS at a faster rate than the SGD.

In Study 2, two (Joe and Saskia) of the four participants reached criterion on use of all three communication options assessed (SGD, PE, and MS), while two participants (Sam and Nicky) did not acquire the use of MS. Three participants (Joe, Saskia, and Nicky) demonstrated a preference for using the SGD, while Sam indicated more proficient use of, and a slight preference for, using PE.

In Study 3, two (lan and Hannah) of the four participants reached criterion on use of each communication mode (SGD, PE, and MS). One participant (Jason) did not reach criterion for PE and MS, but he did reach criterion for SGD. Similarly the other participant (Jack) did not reach criterion for any AAC system, but demonstrated moderate proficiency in SGD and PE use, compared to 
baseline. He did not learn to use MS within the timeframe of the study. Two participants (Jason and lan) showed efficient and effective acquisition of, as well as a preference for using, the SGD. The other two participants (Jack and Hannah) indicated better proficiency of, and a preference for, using PE.

Overall, the results of the three studies presented in this thesis demonstrated that seven of the $12(58 \%)$ participants learnt to use and reached criterion for use of each AAC option. Five participants did not reach criterion for MS. While one participant demonstrated an initial ability to use MS, the remaining four participants indicated either no or minimal learning of MS. Two participants did not reach criterion for $\mathrm{PE}$ and one of these participants did not reach criterion for SGD also. However, both of these latter participants demonstrated some increased proficiency in using the PE and SGD communication modes.

In terms of preference, eight of the $12(67 \%)$ participants appeared to show a preference for using the SGD. Preference for using PE was demonstrated by three out of eight (38\%) participants (PE was only assessed in Studies 2 and 3). Finally, only one out of the 12 (8\%) participants indicated a preference for using MS. If acquisition of, and preference for, PE had been assessed in Study 1 these results could be different.

The following sections will discuss the findings of the three studies in terms of acquisition, preference, implications and applications, limitations, and directions for future research.

\section{Acquisition}

The first research question was whether there would be differences in how efficiently and effectively children with ASD and DD learnt to use SGDs, PE, and MS when teaching an initial requesting skill. Results across the three studies suggested that there were differences in how efficiently and effectively the participants learnt to use each communication option. In particular, there appeared to be greater differences between aided and unaided modes of communication, with SGD and PE use being learnt at a faster rate than MS use. This finding is consistent with a few other studies that have reported that aided AAC systems were acquired at a slightly faster rate than unaided systems (Adkins \& Axelrod, 2001; Anderson, 2002; Gregory et al., 2009; lacono \& Duncum, 1995; lacono et al., 1993; Rotholz et al., 1989; Sigafoos \& Drasgow, 
2001; Tincani, 2004). However, it is not clear that the differences in acquisition rates could be seen as as clinically significant and would hold for other children or when teaching other communication skills. Other researchers comparing the acquisition of selection-based (graphic-mode aided) versus topography-based (MS unaided) AAC systems have found evidence for the superiority of MS, but these studies focused on teaching tacting and intraverbal relations, rather than requesting (Sundberg \& Sundberg, 1990; Wraikat et al., 1991).

There are several possible explanations for these differences in learning between SGD, PE, and MS that were found in the present series of studies. First, MS may be a more difficult AAC system to learn because, while graphic symbols used for the SGD and PE options require only recognition memory, MS requires the use of recall memory, which may be more demanding on the children's working memory (lacono \& Duncum, 1995; lacono et al., 1993). Further, for some children with ASD and DD who are reported to have fine motor and imitation skills deficits (Blischak, Loncke, et al., 1997; lacono \& Duncum, 1995; Seal \& Bonvillian, 1997), forming manual signs might involve greater response effort than pointing to and touching an icon on a SGD or handing over a picture card as is required when using PE. However, none of the children involved in these three studies appeared to have any motor deficits as determined by the Vineland-II assessment (Sparrow et al., 2005).

Second, it could be that MS is a more difficult system to teach or does not lend itself as readily to teaching via graduated guidance. This explanation is consistent with the fact that the intervention procedures were modified for four out of the 12 participants in an effort to promote greater learning. Specifically, two participants (Zac, Study 1; Jack Study 3) received mass training trials (10 consecutive graduated guidance trials with no time delay and no reinforcement), which did not lead to criterion for MS. A least-to-most prompting procedure implemented for one participant (Tom, Study 1 ) also did not result in acquisition. Manipulations to time delay and reinforcement did not lead to acquisition for one participant (Sam, Study 2), but, after differential reinforcement (prompted trials were not reinforced) was introduced to another participant (Tom, Study 1) criterion for MS was achieved. The results of these modifications suggested that 
some instructional procedures for some students may be better suited to teaching MS than others.

Thirdly, participant motivation and preferences could have been a factor in the generally slower rates in acquisition of the MS option. Apart from one participant (David, Study 1); the children did not demonstrate a preference for using MS and were therefore perhaps less motivated to learnt to use it. Preference, or lack of preference, may influence motivation to learn to use an AAC option (see Preference for further discussion).

The relatively more rapid acquisition of the SGD option deserves comment as it suggests that this system was either easier to learn, easier to teach, and/or was associated with greater motivation (preference). The results with respect to the SGD intervention provide empirical evidence for the successful use of the Proloquo2Go ${ }^{\text {TM }}$ (Sennott \& Bowker, 2009) software application run on iPods ${ }^{\circledR}$, iPhones $₫$, and $\mathrm{Pads} \otimes$ to serve as AAC systems. Consistent with initial evidence (Kagohara et al., 2010; van der Meer, Kagohara, et al., 2011), the children in these three studies successfully acquired use of this AAC system. However, the children did appear to have some initial difficulties in learning to press the target symbols with sufficient finesse to activate the speech-output. With practice, and some variations to the teaching procedures for several participants, all 12 children reached criterion or showed an increased proficiency over baseline with respect to the use of the SGD. Specifically, four out of the 12 participants required some procedural modifications for the SGD. The use of mass training trials for one participant (Zac, Study 1) and a $0 \mathrm{~s}$ time-delay for another participant (Sam, Study 2) were both found to be successful in promoting acquisition criterion of the SGD. Similarly switching to an iPad® (Jack, Study 3 ) with a larger screen appeared to enable one participant to successfully select the correct symbol with sufficient finesse to activate the voice-output on a higher percentage of trials. For one participant (Joe, Study 3) slow SGD acquisition appeared to be due to symbol discrimination difficulties, which were also evident in the PE option. Removal of the distractor symbol led to successful SGD use (see Limitations for further discussion).

A total of eight participants were taught to use PE. Only one participant (Joe, Study 3) required modifications to the procedures in order to reach criterion 
and this appeared to be due to difficulties in the discrimination of graphic symbols (see paragraph above). In accordance with previous research (Flippin et al., 2010; Hart \& Banda, 2010; Lancioni et al., 2007; Preston \& Carter, 2009; Schlosser \& Wendt, 2008; Sulzer-Azaroff et al., 2009; Tien, 2008; Wendt, 2009) the present results suggested that PE can be efficiently and effectively taught to individuals with ASD and DD. Together, results from the three studies indicated that for the most part the differences in speed of acquisition and proficiency of AAC system use were more significant in Study 1 when only SGD and MS were compared, than in Studies 2 and 3 when all three AAC systems (SGD, PE, and MS) were compared. Despite MS consistently being learnt at slower rates than SGD and $P E$, the idiosyncratic variability in acquisition rates and proficiency between all three AAC modes (SGD, PE, and MS), when considered in light of previous research (Chapter 3 ), further highlight the point that there may not be one single mode of AAC that is best for all children with ASD and DD. For this reason, it would seem important to base decisions regarding which AAC option to implement upon assessment of the individual's acquisition data, abilities, and needs, as well as their preferences for using different AAC systems (Sigafoos \& Drasgow, 2001).

\section{Preference}

The second research question was whether children would show a preference for using SGDs versus PE versus MS. Results across the three studies indicated that all 12 participants appeared to demonstrate a preference for one AAC system over the others. Specifically, $67 \%$ of participants indicated a preference for using the SGD, 38\% exhibited a preference for using PE, and only one participant indicated a preference for using MS. The present studies appear to be the first to utilise a structured choice-making paradigm (Sigafoos, 1998) to compare preferences for aided versus unaided AAC. Due to their inherent differences in response topographies, MS cannot be presented in the same way as SGD and PE (Sigafoos et al., 2005). MS was therefore represented by a photo of the trainer signing (Studies 1 and 2) or line drawings of the hand formations needed to sign (Study 3). Because one participant (David, Study 1) demonstrated a preference for MS and several participants occasionally selected the MS option in the device preference assessments, this approach might be seen as a viable 
means of representing the unaided (MS) AAC choice. Further, several participants regularly pointed to the photo/line drawing corresponding to the sign they then formed, suggesting that they might have understood that the photo/line drawing represented the MS option. Overall it could be concluded that the choicemaking procedure used in these three studies was largely successful in providing individuals with an opportunity to express their preferences for using one AAC system over another, enabling them to gain some control and autonomy over the intervention process, and thereby engaging in what might be seen as selfdetermined behaviour (Sigafoos, 2006).

The third research question was: If children with ASD and DD do show a preference for one AAC mode above the others, when does this preference first emerge; that is, does preference first emerge prior to or only after acquisition training? Studies 1 and 2 only assessed preferences for AAC modes as participants began to learn each communication system. Five (Tom, Zac, and Eli, Study 1; Saskia and Nicky, Study 2) out of the eight participants across these two studies demonstrated a stable preference for one AAC mode above the other/s throughout the intervention. For example Eli (Study 1) and Saskia (Study 2) consistently chose the SGD on each opportunity throughout every phase of the study. The remaining three participants (David, Study 1; Joe and Sam, Study 2), however, exhibited variation and change in their preference for using one AAC system over another as they developed proficiency with using each system. For example, initially David (Study 1 ) indicated a preference for using MS, but as he gained proficiency with using the SGD he started choosing this AAC mode. Similarly, Joe (Study 2) switched between choosing the SGD and PE as he gained proficiency with each of these AAC options. Except for one participant (Saskia, Study 2), acquisition effect sizes were highest for each child's preferred AAC mode, suggesting participants consistently preferred the communication option which they were proficient at using.

However, it remained unclear whether preference for, or acquisition of, AAC systems emerged first; that is: does preference lead to better acquisition or does ease in acquisition lead to preference? This also related to the fourth research question: Does preference influence how efficiently and effectively children learn to use AAC? It was unclear whether valid indicators of preference 
could in fact be assessed prior to some level of exposure to each device. Study 3 , therefore, implemented AAC preference assessments before baseline, during baseline, and throughout intervention as well as the remaining phases of the study. Results from these assessments indicated that, for some participants, preference was evident before acquisition training, while for other participants stable preferences only emerged after intervention resulted in acquisition. Although three (Jason, lan, and Hannah) of the four participants in Study 3 indicated an initial preference for using the SGD, three participants (Jason, Jack, and Hannah) also demonstrated variation in their choices for different AAC systems, with preferences changing as they learnt to use each device. Similarly, an emerging pattern throughout out all three studies indicated that some participants (Eli, Study 1; Saskia, Study 2; Ian, Study 3) who showed an initial and lasting preference also acquired proficient AAC system use relatively rapidly, compared to other participants (David, Study 1; Joe and Sam, Study 2; Jason, Jack, and Hannah, Study 3) who demonstrated variability in their preferences and learnt to use each AAC system at a slower pace and with less proficiency.

These findings shed some light on the previously asked question: Even when clear preference emerges, why do children prefer one AAC system over another? It was hypothesised that children may indicate an immediate preference based (perhaps) on some inherent feature(s) of their preferred AAC option (e.g., voice-output or dynamic displays), but as they gained proficiency in using each device their preferences might change to reflect other features, such as ease of use. For example, Hannah (Study 3) consistently chose the SGD throughout baseline, but demonstrated more efficient and effective acquisition of PE during intervention and she then also switched to choosing PE, instead of the SGD, throughout the remaining phases of the study. One interpretation of these findings is that preferences for SGD could have been based upon instant appeal whereas preferences for PE could have been based upon ease of use. This could also explain that the initially slower rates for learning and lesser preference for the MS option might be due to it having less instant appeal. If so, this might have meant that some children were less motivated to learn to use the MS option. lacono and Duncum (1995) also hypothesised that children may not prefer to use MS over other SGD- and/or PE-based systems due to the increased motor 
demands associated with using MS. They posited that the SGD was more preferred in their study because it was a motorically simpler way of communicating. However, it remains unclear if the present results and those of lacono and Duncum suggest that proficiency (how successfully a participant was able to use the AAC systems) is related more to preference than ease and speed of learning.

The fifth research question was: Are preferences stable over time? Six (Tom, Zac, and Eli, Study 1; Saskia and Nicky, Study 2; lan, Study 3) out of the 12 participants demonstrated a seemingly instant and lasting preference for one AAC device (the SGD) over the others. For three other participants (Sam, Study 2; Jack and Hannah, Study 3) a stable preference for one AAC system (PE) over the others emerged once they acquired some (high) level of proficiency with that AAC system. Three participants (David, Study 1; Joe, Study 2; Jason, Study 3) never appeared to have shown stable preferences in that their choices for the AAC options varied across sessions even during follow-up. For example, although Jason (Study 3) demonstrated an overall preference for the SGD prior to follow-up, his preference shifted to PE during the follow-up phase and he also performed at a higher rate with PE, than with the SGD, during the follow-up phase. These results suggest that for some children, preferences for different AAC modes may change over time and such changes might be influenced by their performance, as well as influence their performance. Thus, there might be value in periodically reassessing children's preferences after intervention, even when they showed a preference for a particular AAC mode during acquisition and immediately after acquisition.

One conclusion based on the aforementioned findings is that participants should receive equal exposure to, and achieve equal proficiency with, each communication system prior to implementing the choice assessment. This equalisation of exposure and proficiency may be necessary in order to ensure participants are making an informed choice. This may also be necessary for ensuring that any such preference for one device over another was not due to differences in performance (Sigafoos et al., 2005; Son et al., 2006; Soto et al., 1993). However, this approach may have the disadvantage of not enabling one to determine when a child's preference for one AAC mode over another might first 
emerge. Early identification of preferences would seem important because it might allow for assessment of why preferences might emerge and whether such preferences lead to improved therapeutic outcomes. Incorporating preference assessments prior to acquisition and throughout each phase of the study was intended to provide some insight into when preferences first emerged and why children might exhibit preferences for certain AAC systems, as well as whether preference influenced intervention outcomes. However, from the preference assessments implemented throughout each phase of the present studies, it appeared that for some participants valid inferences of preference could only be made after acquisition of AAC modes. Therefore, although the present approach provided some insight into when preferences first emerged, why certain AAC options were preferred, and how preference influenced intervention progress (as discussed above), from a clinical perspective it might only seem necessary to assess preferences once children have acquired AAC system use.

Further, with respect to this practical application of the choice-making assessment of AAC preferences, the review of the literature (Chapter 3 ) raised the issue of how many choice opportunities are required to enable the demonstration of a preference for one mode of communication over another. With significant variation in the number of preference assessments in previous research (Cannella-Malone et al., 2009; Sigafoos, Green, et al., 2009; Sigafoos et al., 2005; Son et al., 2006; Soto et al., 1993; Winborn-Kemmerer et al., 2009) reliably defining a percentage of selections for which an item can be considered preferred could not be made. The results from the present studies suggest that the issue of the number of preference assessments might not be as important as when these assessments are conducted. On that note, the results suggest that such preferences might be reliably assessed, and remain stable, only after children have learned to use each communication system to some (high) level of proficiency.

The sixth and final research question was: Does preference influence how well the child maintains initial communication skills? In each of the three studies participants who reached follow-up consistently demonstrated better maintenance with their preferred AAC mode, suggesting that preference does influence maintenance of communication skills. While it could be argued that 
early acquisition and continued proficiency with that AAC mode predicted maintenance better than preferences did (e.g., all participants in Study 1), especially considering participants gained more experience with their preferred mode during the post-intervention phase, some of the results would suggest otherwise. First, in Study 1, Zac did not receive post-intervention, but still demonstrated better performance with his preferred AAC option in follow-up. Second, in Study 2, Joe and Saskia exhibited slightly slower (although probably not clinically significant) acquisition of their preferred AAC option (SGD), but demonstrated better post-intervention and follow-up performance with this option, further verifying the idea that preference does influence maintenance of requesting skills.

From an applied perspective, one might also argue that assessing preferences for using one AAC system over another is important in its own right because opportunities to do so might promote self-determination and improve quality of life. For example, researchers have argued that there are aspects of self-determination (e.g., establishing some control and autonomy over one's own life) that are an integral aspect of human development and therefore should not be denied to any individual, whether they have a disability or not (Stancliffe, 2001; Wehmeyer, 1992). Additionally, it has been argued that allowing individuals to self-determine aspects of the intervention process, for example, choosing which AAC option they would like to use, might positively influence on their willingness to participate in intervention and boost their motivation to learn to communicate (Arthur-Kelly et al., 2007; Sailor et al., 1988; Sigafoos, 2006). Congruent with previous research (Cobb et al., 2009; Sailor et al., 1988), incorporating elements of self-determination, such as choice-making and assessing personal preferences (Wehmeyer, 1992; Wehmeyer \& Shogren, 2008; Wehmeyer et al., 2010), did appear to positively influence learning and continued use of the targeted skills among some of the participants in this series of three studies. Generally, participants across all three studies demonstrated greater proficiency with, and better maintenance of, their preferred AAC mode.

The concept of self-determination, introduced in Chapter 3 , has provided part of the rationale for why one might want to enable participants to choose among the three AAC options. In addition, the studies reported in this thesis did 
not directly test the merits (validity) of self-determination or whether providing choice per se is important. Empirical evidence to support the significance of self-determination would require different types of experimental manipulations than the ones employed in the present three studies. For example, the issue might be addressed by systematically manipulating the provision of choice (i.e., the learner chooses the AAC option versus having the option chosen by the communication partner) to assess whether this influenced acquisition and maintenance.

\section{Implications and Applications}

The findings presented in the present thesis have several implications and applications for research and practice. As previously outlined (see Preference) a key component of the research was to promoteaspects of self-determination, in particular tailoring the intervention to incorporate children's preferences. This was evident not only in terms of assessing which AAC option children preferred to use for requesting-based communication skills, but also in terms of assessing preferences to identify items that the children were taught to request. To this end, a systematic two-stage stimulus preference assessment (Green et al., 2008; Verschuur et al., 2011) was implemented to identify toys and/or snacks that participants appeared to prefer and would be appropriate to teach the requesting skills. Although some of the selection percentages for preferred stimuli could be interpreted as relatively low to be qualified as preferred (e.g., Study 1, playing on the computer $=30 \%$ for Zac), nearly all of the 12 participants consistently requested, selected, and then played with/consumed the toys or snacks identified through this process. Anecdotally, but perhaps not surprisingly, in Study 3 it was also reported that AAC-based requesting (regardless of the mode) was better for items that appeared to be most reinforcing. For example, Hannah selected $M \& M \otimes$ candy $50 \%$ of the time (this was the highest percentage of selections across all items assessed) and learnt to request this item most efficiently and effectively across all three communication systems. Only one participant (Jack, Study 3) did not demonstrate consistency in requesting, selecting, and playing with/consuming the presumed preferred stimuli. These findings suggest that teaching/learning AAC system use (regardless of the mode) for requesting was potentially dependent on the use of powerful reinforcers. 
It is generally recognised that children with ASD and DD appear to be more motivated to learn when highly reinforcing items are used (Drasgow et al., 2009; Lovaas, 2003). For this reason, requesting (manding) is often the first communicative skill targeted for instruction. Requesting is also indicated because such responses are of direct benefit to the speaker (Skinner, 1957) and can therefore be seen as a more instrumental communicative act than, for example, naming (tacting), which is of benefit to the listener and hence a more socially oriented communicative function. For children with ASD and related DD, who have impaired reciprocal social interaction (American Psychiatric Association, 2000), the motivation for requesting-based communication would be predicted to be higher than the motivation for learning more socially oriented forms of communication, such as tacting (Kagohara et al., 2012). Recent research identified differences in the brain configuration and activity of individuals with ASD compared to individuals without ASD (e.g., Casanova, 2007; Casanova, Buxhoeveden, \& Brown, 2002; Geschwind, 2009). While preliminary, findings from these studies implied that some individuals with ASD may only focus their attention to stimuli which are of interest and highly motivating to them. Together these outcomes highlight the importance of promoting self-determination, where incorporating children's preferences (not only for the AAC system used, but for the items participants learnt to request) into the intervention process can positively influence progress in learning.

However, in some instances, it could be argued that incorporating child preferences for different AAC options might not be desirable. For example, a child's preference for using one AAC option over the others may not coincide with the clinician/teacher/parent's choice. This was the case for David (Study 1) who demonstrated a preference for using MS. His parents, on the other hand, indicated a preference for him to learn the SGD because they believed it to be a more widely understood communication device that would allow David to expand on his communication capabilities. The value of David's preference was therefore compromised, especially considering David's continued communication intervention subsequent to the study implemented in the present thesis focused on SGD use only. With the present results and previous research (Soto et al., 1993) indicating that learning and skill acquisition might be directly related to 
choice-making and preference, it is maintained that participant's preferences should not be underestimated.

Nonetheless, discriminative use of aided and unaided AAC (Reichle \& Ward, 1985; Sigafoos \& Drasgow, 2001) may be an effective compromise to resolving such issues and applying the present findings to everyday contexts for individuals with ASD and DD. This approach could be utilised by incorporating the child's preferred AAC option into certain communicative exchanges, while teaching conditional use of other AAC options (that the children have already learnt to use) depending on the context. For example, it may be more efficient to use PE to indicate a desired item in a noisy restaurant than SGD with synthesised speech-output that may be difficult to hear in such environements. Similarly, MS might not be effective in situations where the communicative partner does not understand sign language. In other cases, the decision to use aided or unaided AAC may be dependent on whether the aided device is accessible and working. These examples illustrate how effective communication across a range of contexts can be dependent on the individual being able to use multiple AAC modes in order to attain vocabulary flexibility, functionality, and system intelligibility (Sigafoos \& Drasgow, 2001). Additionally, it has been hypothesised that PE systems, for instance, may have greater effects on social interaction skills than other systems, while SGDs may have more impact on verbal skills (Ganz et al., 2011). This suggests that multimodal AAC use could also promote development in other areas of functioning, although data to support such claims are currently lacking. This would therefore seem an obvious area for future research.

With respect to choosing between MS, PE, and SGD, advances in SGD technology might tip the balance towards these devices if the advances were to allow for greater communicative independence or were shown to be more socially valid and less stigmatising (Mirenda, 2009). This thesis could be seen as providing timely empirical evidence to support the applicability and successful use of a new emerging technology ( $(\mathrm{Pod} \circledast / \mathrm{iPad} \circledast$-based $S G D s)$ as communication aides for children with ASD and DD. If the use of such SGDs becomes further supported by empirical data, and thus more confidently recommended by professionals, there will be a need for teachers, parents, and 
clinicians to become proficient with using this technology (van der Meer, Kagohara, et al., 2011). Furthermore, given that parents and teaching staff are arguably the children's more important communicative partners it would seem essential that they also become competent in teaching individuals the AAC modes that the child is capable of learning and interested in using.

Because trained research staff implemented AAC system use in Studies 1 and 2 , the practicality as well as applicability of these findings to the general classroom and home settings were unclear. Therefore, Study 3 sought to assess whether parents and teaching staff could effectively learn to implement the intervention procedures in order to extend the applied relevance of the research findings. Procedures (including written step-by-step instructions, explaining these instructions, and providing regular feedback) consistent with best practices for training parents and teaching staff to implement behaviourally-oriented teaching programs (Reid et al., 2011) appeared to be successful, as evidenced by generally positive results in terms of AAC system acquisition and the high degree of procedural integrity. This is an important finding as it would seem to greatly extend the applied relevance of the intervention procedures utilised in the three studies of this thesis.

\section{Limitations}

There were several limitations to the studies presented in this thesis that should be considered when interpreting the findings. First, as is characteristic of studies with small numbers of participants using single-case experimental designs, the external validity or generalisability of the findings remains to be determined by systematic replication (Sidman, 1960). While the 12 children in these three studies provide a fairly consistent picture of results, further research involving additional participants is needed to determine whether these findings can be replicated with other children who require AAC intervention.

A second related limitation was that generalisation data to other contexts, people, and communicative forms were not collected. All three studies focused only on teaching requesting (manding). The effects of teaching more complex communication functions, such as tacting and intraverbal behaviour (Skinner, 1957) on acquisition rates and preferences for each of the three AAC systems (SGD, PE, and MS) remains unknown (see Future Research). 
Along these lines, high levels of inter-observer agreement (above the generally agreed criterion of a minimum of $80 \%$ agreement; Kennedy, 2005) were obtained for each of the three studies included in the present thesis. These results could be explained by the small number of simple communicative responses, that were easy to interpret as occurring or not occurring. If more complex language (e.g., involving a sequence of signs for the MS option) were taught, it would seem appropriate to provide a more detailed account of interobserver agreement.

Fourth, while use of the Vineland-II (Sparrow et al., 2005) provided a good assessment of expressive language age equivalence, thereby insuring that the communication intervention was appropriate for each participant's language abilities, results would have been strengthened by including further assessments (see Future Research) and diagnostic information. For example, parents and/or teachers provided diagnostic information, but evidence about how this was achieved (e.g., by what type of professional, using what reliable and valid instrument) was not obtained. Attaining this information or using valid instruments (such as the ADOS and ADI-R) to diagnose participants prior to the intervention would have improved the value of the research outcomes, but was beyond the scope of the present research and competencies of the author.

Fifth, in order to compare the relative efficacy of two or more communication systems it is crucial to develop functional equivalence between each AAC system (Schlosser, 2003c). Both Studies 1 and 2 failed to develop equivalency between the AAC systems assessed (while the SGD option in Study 1 included symbols for SOCIAL INTERACTION, SNACK, and PLAY, the MS option only had two photos to represent SNACK and PLAY; while the PE option in Study 2 required a two-step response in which participants had to place the I WANT and SNACK/PLAY symbols onto the card provided, the SGD and MS options only required a one-step response). Consequently, differences in response demands of each communication system may have influenced results in terms of both intervention outcomes and preferences (Ringdahl et al., 2009; Winborn-Kemmerer et al., 2009). Therefore, it was ensured that all three communication systems in Study 3 were as similar as possible in terms of creating functional equivalence. Because results of the third study were 
comparable to results of the first and second studies it is possible that any such effects of non-equivalency were minimal. There may also be value in representing each system in an equivalent format, such as a laminated card with a photo to represent each option. In this way the discriminative stimulus for each option would be the same allowing for a less biased indication of preference (i.e., the SGD and PE are naturally occurring discriminative stimuli that are present in the environment and associated with reinforcement while the photo/diagram of MS is not, perhaps influencing preference). However, due to the inherent differences in response topographies of MS versus SGD and PE, a further limitation of each study was that a MS response could be emitted during SGD and PE sessions, but not vice versa. This meant that MS was on a more intermittent schedule of reinforcement relative to the SGD and PE, also potentially influencing both rapidity of acquisition, and preferences. This could be overcome by having all AAC systems available in each session; however, this might prevent bringing each system under stimulus control and prevent individual acquisition of each AAC option.

Similarly, different reinforcement histories (prior to implementation of the present studies) with each AAC system provide a fourth possible limitation of the studies. For example, previous research has suggested a facilitative effect of topography-based (MS) communication on the acquisition of a selection-based (PE, SGD) system (Shafer, 1993). Likewise it could be hypothesised that previous unsuccessful MS training might negatively influence current preferences for that communication system. It appeared that all 12 participants had some experience with at least one of the AAC systems (SGD, PE, or MS) assessed in the studies, which might have influenced the present results. However, due to the subjective and anecdotal nature of the information provided, the precise amount and nature of such prior experience was unknown. It is also inevitable (and therefore difficult to control) that children with little or no speech have some history with AAC that may bias results in terms of acquisition and preference for AAC options. In order to minimise such biases, as well as potential novelty effects of certain AAC systems (e.g., SGD) the same number of sessions/reinforcement for each communication option was maintained during acquisition training (except where modifications to the instructional procedures 
were needed for certain participants to increase performance) in each of the three studies.

Seventh, in Study 1 two participants (David and Eli) developed perseverative requesting (i.e., making repeated requests, even after being informed to wait for the requested item or when the item is not being offered). For example, Eli frequently touched all of the icons on the SGD repeatedly. Because the definition of a correct response was independently hitting the correct icon (PLAY in the case of Eli) within 10 seconds it is possible that he did not learn one discrete functional response (the request PLAY), but rather, it is possible that he learnt the chain of randomly touching icons until the desired reinforcer was delivered. While this did not appear to occur for other participants in Studies 1 and 2, in Study 3 this limitation was accounted for by defining a correct response as independently using the $A A C$ system to request the specific item being offered. If the participant requested a different item to the one being offered, the response was immediately corrected (error correction procedure) by modelling the correct response but counting it as incorrect.

Finally, the three studies utilised a combined multiple-baseline and alternating-treatments single-case design (Kennedy, 2005). The multiple-baseline is an effective approach for demonstrating acquisition of communication skills across participants. This design is commonly viewed as ethically more desirable than A-B-A-B type designs because the treatment is not withdrawn (Barlow \& Herson, 1984); it would also be difficult to unlearn the newly acquired communication skills. However, use of the alternating-treatments design to compare efficacy of each AAC system could be interpreted as a weakness of the present research. When several acquisition interventions (e.g., SGD, PE, and MS) are used to teach the same behaviour (e.g., requesting to play), a conclusion regarding which treatment was responsible for the behaviour change cannot be made with as much confidence due to the possibility of multiple treatment interference or carry over effects (i.e., the influence a preceding intervention has on the effectiveness of a succeeding intervention; Schlosser, 2003d). A better demonstration of experimental control might have been achieved through the use of an adapted alternating-treatments design (Sindelar, Rosenberg, \& Wilson, 1985). In this latter design, several different dependent variables are equated 
along a number of dimensions and assigned to several different acquisition interventions (independent variables). In the present research, for example, three groups of preferred stimuli (that participants would be taught to request) could have been equated along several dimensions (preference, type of item - snacks versus toys, effort to perform the manual sign). One group would be assigned to the SGD condition, one to PE, and one to MS, thereby allowing for comparison of how efficiently and effectively each AAC option is acquired. Such an adapted alternating-treatments design was not implemented in the present studies because children were at the beginning stages of intervention. It was deemed more important to limit the amount of vocabulary introduced so that the children were not being taught too many new skills at one time, which could potentially inhibit progress in learning, as well as negatively influence preferences for the AAC modes.

\section{Future Research}

In light of the results from the three studies presented in the present thesis, several directions for future research can be suggested. Although the studies presented in the present thesis utilised a simple questionnaire as well as a standardised scale (Vineland; Sparrow et al., 2003; Sparrow et al., 2005) to assess each participant's adaptive behaviour functioning, including communicative functioning, future research (and practice) might benefit from inclusion of a more formal pre-intervention assessment of each child's specific communication and related abilities (Light et al., 1998). For example, a comprehensive assessment of fine and gross motor skills might be helpful to verify whether participants have the dexterity to perform the physical movements required for each AAC system. It might also be beneficial to determine whether children are at a pre-symbolic or symbolic stage of language development (Paul, 2008; Reichle \& Brady, 2012; Rowland, 2009; Schuler \& Prizant, 1987). If children do demonstrate symbolic language, for example, it would seem important to assess whether they are able to make conditional discriminations (e.g., matching-to-sample skills; Shafer, 1993). It would also be of potential benefit to determine the level of iconicity of graphic symbols or manual signs that is appropriate for individuals (Koul et al., 2001). This is particularly relevant considering that some participants in Studies 1 and 2 (e.g., Eli, Study 1 and Joe, 
Study 2) appeared to have difficulties in learning to discriminate between the graphic symbols (line drawings) used in the SGD and PE systems, while participants in Study 3 did not demonstrate such problems, perhaps because more iconic photographs were used. Future research is required to determine whether photographs of specific items might facilitate the acquisition of SGD- and PE-based requesting when compared to the use of line drawings (De Paul \& Yoder, 1986; Johnston \& Cosbey, 2012).

Further, comprehensive pre-intervention communication assessments might be indicated and useful in providing indicators of the skills and characteristics of children with ASD and DD that predict which AAC system is likely to be learnt fastest and preferred by individual children. That is, future research could assess predictors of success, such as learner characteristics or environmental features that may allow the prescription of devices with speechoutput for learners with certain characteristics and avoid such systems for individuals who do not meet those characteristics (Schlosser et al., 2003). It would also be interesting to assess whether these predictors match children's preferences for one AAC system over another. If so, this could allow for further understanding of why children might prefer certain AAC systems, as well as potentially contribute to development of better guidelines to assist clinicians in selecting an appropriate AAC option for individuals with ASD and DD.

With the use of a suitable AAC mode/s that the individual user appears to prefer, future research could focus on teaching more complex social communication, including spontaneous initiated communication (instigating and maintaining conversations, commenting on the environment, and answering questions). This is particularly relevant considering clinical experience suggests that increased communicative initiation leads to greater participation in activities, and increased self-determination (Erwin \& Brown, 2003). The present research and the majority of previous research on AAC system acquisition (e.g., Lancioni et al., 2007; van der Meer \& Rispoli, 2010) has focused on teaching a single communicative function, such as requesting (manding) using systematic instructional procedures based on the principles of ABA (Duker et al., 2004), including time-delay, prompting, and reinforcement. Because the present instructional strategies utilised a verbal prompt (e.g., Let me know if you want 
something) to initiate the requesting trials, it is possible that the participant's responses were at least partially under the control of the speech of the communication partner. Anecdotally, some participants (e.g., lan, Study 3) did demonstrate spontaneous communicative requesting during follow-up sessions (i.e., requested items before the instruction was given). Future research could evaluate the effects of a refinement of the present instructional procedures so as to develop more spontaneous communication (Sigafoos, Drasgow, et al., 2009). Although Study 3 taught a more complex requesting repertoire (specific requesting), future research could increase this requesting repertoire, and sequentially introduce other units of language outlined by Skinner (1957), such as naming (tacting) and initiating and responding to questions and other aspects of conversation (intraverbals). Introduction of such a language program could be interspersed with opportunities for the child to practice their already learned requesting repertoire so as to maintain the motivation to partake in such social communicative exchanges, because such social interaction skills do not seem to be readily acquired by individuals with ASD and other DD (American Psychiatric Association, 2000). As suggested by lacono and Duncum (1995), another area of research would be to determine whether the use of AAC may facilitate such language development or whether it simply provides a means to express underlying linguistic skills. While one case study, for example, has indicated SGD use may be a mediator for expression of language that the participant already understood (Cheslock, Barton-Husley, Romski, \& Sevcik, 2008), further research is required to extend these findings.

It also remains unclear whether preference for an AAC modality will influence learning of other communication skills. Future research questions could include: Does preference influence how efficiently children with ASD and DD learn new and more advanced communication skills? Further studies could provide $A A C$ intervention (with $S G D, P E$, and $M S$ ) for simple requesting, while assessing each participant's preferences for one communication option over another and then teach the same participants more complex social communication skills with each AAC system, while still assessing preferences. This would allow for better determination of how preference influences acquisition. While it is expected that preference would facilitate acquisition of 
more complex language, it is also possible that preferences could change to match the AAC system with which the participant most easily learnt the new skills or change to match the system that is most efficient for more advanced communicative exchanges. For example, when teaching conversational turntaking, children who preferred using an $\mathrm{PPod} 囚$-based SGD when requesting, might start to prefer using MS if the latter mode proved to be more efficient in scenarios that required numerous conversational turn-taking.

Finally, future research would benefit from social validity data of parents/teachers/clinician's perceptions on the usefulness of the AAC intervention and whether the student's social and communicative behaviours improved with intervention, as well as the practicality and utility of the preference assessment. Further, it would be interesting to assess whether parent's/teacher's/clinician's preferences for one AAC mode over another matched that of the child and if not, how this could be resolved (see also Implications and Applications). Finally, social validity data of the relative efficacy of each AAC system might also help clinicians in the difficult decision of selecting a suitable AAC system for individuals with ASD and DD.

\section{Conclusion}

This thesis aimed to develop and evaluate more effective procedures for implementing AAC intervention for children with ASD and related DD who have limited or no verbal communication skills. ASD and DD were conceptualised as neurological disorders resulting in behavioural excesses and deficits that might be ameliorated to some extent through programmes based on the basic principles of ABA. Using these systematic instructional strategies (time delay, prompting, and reinforcement), results across the three studies indicated that all of the participants successfully learnt to use at least one of three common modes of communication (SGD, PE, and MS) to request highly preferred items to some (high) level of proficiency. The studies simultaneously incorporated aspects of enhancing self-determination by enabling children to express their preferences for using one communication mode over another. Results suggested that the children showed a preference for using one of the particular AAC options. Further, the children demonstrated greater proficiency and better maintenance with their preferred communication option. This finding highlights some potentially 
positive effects of enabling individuals some degree of self-determination with respect to aspects of the intervention they receive. Given the encouraging intervention outcomes for the present series of three studies, future research and practice should continue to seek ways of increasing opportunities for selfdetermination in individuals with ASD and DD. 


\section{REFERENCES}

Accardo, P., \& Whitman, B. (1996). Dictionary of developmental disabilities terminology. Sydney: MacLennan \& Petty.

Adkins, T., \& Axelrod, S. (2001). Topography- versus selection-based responding: Comparison of mand acquisition in each modality. The Behavior Analyst Today, 2, 259-266.

Alberto, P., \& Troutman, A. (Eds.). (2006). Applied behavior analysis for teachers (7th ed.). Upper Saddle River, New Jersey: Pearson Education, Inc. American Psychiatric Association. (2000). Diagnostic and statistical manual of mental disorders (4th ed., text revision). Washington DC: Author.

American Psychiatric Association. (2011). DSM-5 Development. Retrieved 1 February, 2012, from http://www.dsm5.org/Pages/Default.aspx Anderson, A. (2002). Augmentative communication and autism: A comparison of sign language and the Picture Exchange Communication System. Dissertation Abstracts International: Section B: The Sciences and Engineering, 62, 4269.

Arthur-Kelly, M., Bochner, S., Center, Y., \& Mok, M. (2007). Socio-communicative perspectives on research and evidence-based practice in the education of students with profound and multiple disabilities. Journal of Developmental and Physical Disabilities, 19, 161-176.

Arthur-Kelly, M., Sigafoos, J., Green, V., Mathisen, B., \& Arthur-Kelly, R. (2009). Issues in the use of visual supports to promote communication in individuals with autism spectrum disorder. Disability and Rehabilitation, 31, 1474-1486.

Asperger, H. (1944). Die autistischen psychopathen im kindesalter. Archiv fur Psychiatrie und Nervenkrankheiten, 117, 76-136.

Attwood, A. (2008). The complete guide to Asperger's syndrome. London: Jessica Kingsley Publishers.

Azrin, N., \& Armstrong, P. (1973). The "mini-meal" - A method for teaching eating skills to the prfoundly retarded. Mental Retardation, 14, 29-33.

Baer, D., Wolf, M., \& Risley, T. (1968). Some current dimensions of applied behavior analysis. Journal of Applied Behavior Analysis, 1, 91-97. 
Bailey, A., Le Couteur, A., Gottesman, I., Bolton, P., Simonoff, E., Yuzda, E., \& Rutter, M. (1995). Autism as a strongly genetic disorder: Evidence from a British twin study. Psychological Medicine, 25, 63-77.

Barlow, D., \& Hayes, S. (1979). Alternating treatments design: One strategy for comparing the effects of two treatments in a single subject. Journal of Applied Behavior Analysis, 12, 199-210.

Barlow, D., \& Herson, M. (1984). Single-case experimental designs: Strategies for studying behavior change (2nd ed.). New York: Pergamon Press.

Baron-Cohen, S. (2004). Autism: Research into causes and intervention. Pediatric Rehabilitation, 7, 73-78.

Barrett, R., \& Sisson, L. (1987). Use of the alternating treatments design as a strategy for empirically determining language training approaches with mentally retarded children. Research in Developmental Disabilities, 8, 401412.

Bates, E., Benigni, T., Bretherton, I., Camaioni, L., \& Volterra, V. (Eds.). (1979). The emergence of symbols: Communication and cognition in infancy. New York: Academic Press.

Bates, E., Camaioni, L., \& Volterra, V. (1975). The acquisition of performatives prior to speech. Merrill-Palmer Quarterly, 21, 205-226.

Beck, A., Stoner, J., Bock, S., \& Parton, T. (2008). Comparison of PECS and the use of a VOCA: A replication. Education and Training in Developmental Disabilities, 43, 198-216.

Beukelman, D., \& Mirenda, P. (2005). Augmentative and alternative communication: Supporting children and adults with complex communication needs (3rd ed.). Baltimore: Paul H. Brookes Publishing Co.

Blischak, D., Lloyd, L., \& Fuller, D. (1997). Terminology issues. In L. Lloyd, D. Fuller \& H. Arvidson (Eds.), Augmentative and alternative communication: A handbook of principles and practices (pp. 38-42). Boston: Allyn \& Bacon.

Blischak, D., Loncke, F., \& Waller, A. (1997). Intervention for persons with developmental diabilities. In L. Llyod, D. Fuller \& H. Arvidson (Eds.), Augmentative and alternative communication: $A$ handbook of principles and practices (pp. 299-329). Boston: Ally and Bacon.

Bliss, C. (1965). Semantography. Sydney: Semantography Publications. 
Bock, S., Stoner, J., Beck, A., Hanley, L., \& Prochnow, J. (2005). Increasing functional communication in non-speaking preschool children: Comparison of PECS and VOCA. Education and Training in Developmental Disabilities, 40, 268-278.

Boisjoli, J., \& Matson, J. (2009). Principles of applied behavior analysis and language acquisition. In A. Fitzer \& P. Sturmey (Eds.), Language and autism: Applied behavior analysis, evidence, and practice (pp. 23-49). Austin, TX: Pro-Ed.

Bondy, A., \& Frost, L. (1994). The Picture Exchange Communication System. Focus on Autistic Behavior, 9, 1-19.

Bondy, A., \& Frost, L. (2001). The Picture Exchange Communication System. Behavior Modification, 25, 725-744.

Bondy, A., \& Frost, L. (2003). Communication strategies for visual learners. In O. Lovaas (Ed.), Teaching individuals with developmental delays: Basic intervention techniques (pp. 291-303). Austin, TX: Pro-Ed.

Bondy, A., \& Frost, L. (2009). The Picture Exchange Communication System: Clinical and research applications. In P. Mirenda \& T. lacono (Eds.), Autism spectrum disorders and AAC (pp. 279-302). Baltimore: Paul $\mathrm{H}$. Brookes Publishing Co.

Bondy, A., Tincani, M., \& Frost, L. (2004). Multiply controlled verbal operants: An analysis and extension to the Picture Exchange Communication System. The Behavior Analyst, 27, 247-261.

Boyle, C., Boulet, S., Schieve, L., Cohen, R., Blumberg, S., Yeargin-Allsopp, M., . . Kogan, M. (2011). Trends in the prevalence of developmental disabilities in US children, 1997-2008. Pediatrics, 127, 1034-1042.

Brady, N. (2000). Improved comprehension of object names following voice output communication aid use: Two case studies. AAC: Augmentative \& Alternative Communication, 16, 197-204.

Bristow, D., \& Fristoe, M. (1984). Learning of blissymbols and manual signs. Journal Speech and Hearing Disorders, 49, 145-151.

Brunner, D., \& Seung, H. (2009). Evaluation of the efficacy of communicationbased treatments for autism spectrum disorders: A literature review. Communication Disorders Quarterly, 31, 15-41. 
Campbell, C., Davarya, S., Elsabbagh, M., Madden, L., \& Fombonne, E. (2011). Prevalence and the controversy. In J. Matson \& P. Sturmey (Eds.), International handbook of autism and pervasive developmental disorders (pp. 25-35). New York: Springer.

Cannella-Malone, H., DeBar, R., \& Sigafoos, J. (2009). An examination of preference for augmentative and alternative communication devices with two boys with significant intellectual disabilities. AAC: Augmentative and Alternative Communication, 25, 262-273.

Cannella, H., O'Reilly, M., \& Lancioni, G. (2005). Choice and preference assessment research with people with severe to profound developmental disabilities: A review of the literature. Research in Developmental Disabilities, 26, 1-15.

Carr, E., Binkoff, J., Kologinsky, E., \& Eddy, M. (1978). Acquisition of sign language by autistic children: I. Expressive labelling. Journal of Applied Behavior Analysis, 11, 489-501.

Carr, E., \& Durand, V. (1985). Reducing behavior problems through functional communication training. Journal of Applied Behavior Analysis, 18, 111126.

Carter, M. (2001). Using choice with game play to increase language skills and interactive behaviors with children with autism. Journal of Positive Behavior Interventions, 3, 131-151.

Casanova, M. (2007). The neuropathology of autism. Brain Pathology, 17, 422433.

Casanova, M., Buxhoeveden, D., \& Brown, C. (2002). Clinical and macroscopic correlates of minicolumner pathology in autism. Journal of Child Neurology, 17, 692-695.

Centers for Disease Control and Prevention. (2009). National center on birth defects and developmental disabilities. Retrieved February 8, 2010, from http://www.cdc.gov/ncbddd/features/counting-autism.html

Centers for Disease Control and Prevention. (2011). Developmental disabilities. Retrieved February 7, 2012, from http://www.cdc.gov/ncbddd/dd/default.htm 
Chakrabarti, S., \& Fombonne, E. (2001). Pervasive developmental disorders in pre-school children. JAMA, 285, 3093-3099.

Checkley, R., Nodge, N., Chantler, S., Reidy, L., \& Holmes, K. (2010). What children on the autism spectrum have to 'say' about using high-tech voice output communication aids (VOCAs) in an educational setting. Journal of Assistive Technologies, 4, 25-37.

Cheslock, M., Barton-Husley, A., Romski, M., \& Sevcik, R. (2008). Using a speech-generating device to enhance communicative abilities for an adult with moderate intellectual disability. Intellectual and Developmental Disabilities, 46, 376-386.

Cobb, B., Lehmann, J., Newman-Gonchar, R., \& Morgen, A. (2009). Selfdetermination for students with disabilities: A narrative metasynthesis. Career Development for Exceptional Individuals, 32, 108-114.

Cohen, D., Amerine-Dickens, M., \& Smith, T. (2006). Early intensive behavioral treatment: Replication of the UCLA Model in a community setting. Developmental and Behavioral Pediatrics 27, 145-155.

Cohen, J. (1988). Statistical power analysis for the behavioral sciences. Hillsdale, NJ: Lawrence Erlbaum Associates.

Cozby, P. (2004). Methods in behavioral research (8th ed.). New York: McGrawHill.

Cregan, A. (1984). Sigsymbols - A graphic aid to communication and language development. Paper presented at the Curriculum Conference, Cambridge Institute of Education.

Cregan, A., \& Llyod, L. (1990). Sigsymbols. Wauconda, Ill: Don Johnston Developmental, Inc.

De Paul, R., \& Yoder, D. (1986). Iconicity in manual sign systems for the augmentative communication user: Is that all there is? AAC: Augmentative and Alternative Communication, 2, 1-10.

DeLeon, I., \& Iwata, B. (1996). Evaluation of a multiple-stimulus presentation format for assessing reinforcer preferences. Journal of Applied Behavior Analysis, 29, 519-532.

Downing, J. (2009). Assessment of early communication skills. In G. Soto \& C. Zangari (Eds.), Practically speaking: Language, literacy, and academic 
development for students with AAC needs (pp. 27-46). Baltimore: Paul $\mathrm{H}$. Brookes Publishing Co.

Drager, K., Clark-Serpentine, E., Johnson, K., \& Roeser, J. (2006). Accuracy of repetition of digitized and synthesized speech for young children in background noise. American Journal of Speech - Language Pathology, 15, 155-164.

Drager, K., Light, J., Carlson, R., D'Silva, K., Larsson, B., Pitkin, L., \& Stopper, G. (2004). Learning of dynamic display AAC technologies by typically developing 3-year olds: Effect of different layouts and menu approaches. Journal of Speech, Language \& Hearing Research, 47, 1133-1148.

Drager, K., Light, J., \& Finke, E. (2009). Using AAC technologies to build social interaction with young children with autism spectrum disorders. In $\mathrm{P}$. Mirenda \& T. lacono (Eds.), Autism spectrum disorders and AAC (pp. 247278). Baltimore: Paul H. Brookes Publishing Co.

Drasgow, E., Sigafoos, J., Halle, J., \& Martin, C. (2009). Teaching mands to individuals with autism spectrum disorders. In A. Fitzer \& P. Sturmey (Eds.), Language and autism: Applied behavior analysis, evidence, and practice (pp. 135-168). Austin, TX: Pro-Ed.

Duker, P., Didden, R., \& Sigafoos, J. (2004). One-to-one training: Instructional procedures for learners with developmental disabilities. Austin, TX: ProEd.

Durand, V. (1993). Functional communication training using assistive devices: Effects on challenging behavior and affect. AAC: Augmentative and Alternative Communication, 9, 168-176.

Durand, V. (1999). Functional communication training using assistive devices: Recruiting natural communities of reinforcement. Journal of Applied Behavior Analysis, 32, 247-267.

Ecker, C., Marquand, A., Mourao-Mirenda, J., Johnston, P., Daly, E., Brammer, M., . . Murphy, D. (2010). Describing the brain in autism in five dimensions - Magnetic resonance imaging-Assisted diagnosis of autism spectrum disorder using a multiparameter classification approach. The Journal of Neuroscience, 30, 10612-10623. 
Edelson, M. (2006). Are the majority of children with autism mentally retarded? Focus on Autism \& Other Developmental Disabilities, 21, 66-83.

Eikeseth, S., Smith, T., Jahr, E., \& Eldevik, S. (2002). Intensive behavioral treatment at school for 4- to 7-year-old children with autism. Behavior Modification, 26, 49-68.

Erwin, E., \& Brown, F. (2003). From theory to practice: A contextual framework for understanding self-determination in early childhood environments. Infants \& Young Children 16, 77-87.

Feeley, K., \& Jones, E. (2012). Instructional strategies. In S. Johnston, J. Reichle, $\mathrm{K}$. Feeley \& E. Jones (Eds.), AAC strategies for individuals with moderate to severe disabilities (pp. 119-154). Baltimore: Paul H. Brookes Publishing Co.

Ferster, C. (1961). Positive reinforcement and behavioral deficits of autistic children. Child Development, 32, 437-456.

Filipek, P. (1999). Neuroimaging in the developmental disorders: The state of the science. Journal of Child Psychology and Psychiatry, 40, 113-128.

Flippin, M., Reszka, S., \& Watson, L. (2010). Effectiveness of the Picture Exchange Communication System (PECS) on communication and speech for children with autism spectrum disorders: A meta-analysis. American Journal of Speech - Language Pathology, 19, 178-195.

Folstein, S. (2006). The clinical spectrum of autism. Clinical Neuroscience Research, 6, 113-117.

Fombonne, E. (2003). The prevalence of autism. JAMA, 289, 87-89.

Fossett, B., \& Mirenda, P. (2007). Augmentative and alternative communication. In S. Odom, R. Horner, M. Snell \& J. Blacher (Eds.), Handbook of developmental disabilities (pp. 330-348). New York: The Guilford Press.

Fowler, C., Konrad, M., Walker, A., Test, D., \& Wood, W. (2007). Selfdetermination interventions' effects on the academic performance of individuals with developmental disabilities. Education and Training in Developmental Disabilities, 42, 270-285.

Franco, J., Lang, R., O'Reilly, M., Chan, J., Sigafoos, J., \& Rispoli, M. (2009). Functional analysis and treatment of inappropriate vocalisations using a 
speech-generating device for a child with autism. Focus on Autism \& Other Developmental Disabilities, 24, 146-155.

Frith, U. (1989). Autism: Explaining the enigma. Oxford: Blackwell Publishers Ltd. Frost, L., \& Bondy, A. (2002). Picture Exchange Communication System training manual (2nd ed.). Newark, DE: Pyramid Education Products.

Ganz, J., Earles-Vollrath, T., Mason, R., Rispoli, M., Heath, A., \& Parker, R. (2011). An aggregate study of single-case research involving aided AAC: Participant characteristics of individuals with autism spectrum disorders. Research in Autism Spectrum Disorders, 5, 1500-1509.

Geschwind, D. (2009). Advances in autism. Annual Review of Medicine, 60, 367380.

Gillberg, C. (2007). The autism spectrum. In J. Jacobson, J. Mulick \& J. Rojahn (Eds.), Issues in clinical child psychology: Handbook of intellectual and developmental disabilities (pp. 41-60). New York: Springer.

Goldfried, M., \& Merbaum, M. (1973). A perspective on self-control. In M. Goldfried \& M. Merbaum (Eds.), Behavior change through self-control (pp. 3-34). New York: Holt, Rinehart, and Winston.

Goldstein, H. (2002). Communication intervention for children with autism: A review of treatment efficacy. Journal of Autism and Developmental Disorders, 32, 373-394.

Green, G. (1996). Early behavioral intervention for autism: What does research tell us? In C. Maurice (Ed.), Behavioral intervention for young children with autism: A manual for parents and professionals (pp. 29-44). Austin, TX: Pro-Ed.

Green, V., Sigafoos, J., Didden, R., O'Reilly, M., Lancioni, G., Ollington, N., \& Payne, D. (2008). Validity of a structured interview protocol for assessing children's preferences. In P. Grotewell \& Y. Burton (Eds.), Early childhood education: Issues and developments (pp. 87-103). New York: Nova Science Publishers.

Gregory, M., DeLeon, I., \& Richman, D. (2009). The influence of matching and motor-imitation abilities on rapid acquisition of manual signs and exchange-based communication responses. Journal of Applied Behavior Analysis, 42, 399-404. 
Hagopian, L., Long, E., \& Rush, K. (2004). Preference assessment procedures for individuals with developmental disabilities. Behavior Modification, 28, 668-677.

Halle, J., Marshall, A., \& Spradlin, J. (1979). Time delay: A technique to increase language use and facilitate generalization in retarded children. Journal of Applied Behavior Analysis, 12, 431-439.

Hart, S., \& Banda, D. (2010). Picture Exchange Communication System with individuals with developmental disabilities: A meta-analysis of single subject studies. Remedial \& Special Education, 31, 476-488. doi: 10.1177/0741932509338354

Hehner, B. (1980). Blissymbols for use. Toronto: Blissymbolics Communication Institute.

Hill, E., \& Frith, U. (2004). Understanding autism: Insights from mind and brain. In U. Frith \& E. Hill (Eds.), Autism: Mind and brain (pp. 1-20). New York: Oxford University Press.

Horner, R., \& Baer, D. (1978). Multiple-probe technique: A variation of the multiple probe baseline. Journal of Applied Behavior Analysis, 11, 189196.

Horovitz, M., \& Matson, J. (2010). Communication deficits in babies and infants with autism and pervasive developmental disorder-not otherwise specified (PDD-NOS). Developmental Neurorehabilitation, 13, 390-398.

Howlin, P., Gordon, R., Pasco, G., Wade, A., \& Charman, T. (2007). The effectiveness of Picture Exchange Communication System (PECS) training for teachers of children with autism: A pragmatic, group randomised controlled trial. The Journal of Child Psychology and Psychiatry, 48, 473-481.

Iacono, T., \& Duncum, J. (1995). Comparison of sign alone and in combination with an electronic communication device in early language intervention: Case study. AAC: Augmentative and Alternative Communication, 11, 249259.

lacono, T., Mirenda, P., \& Beukelman, D. (1993). Comparison of unimodal and multimodal AAC techniques for children with intellectual disabilities. AAC: Augmentative and Alternative Communication, 9, 83-94. 
Iversen, P. (2007). The informative pointing method. Retrieved 16 July, 2010, from www.strangeson.com

Jeanes, R., Reynolds, B., \& Coleman, B. (1989). Dictionary of Australasian signs (2nd ed.). Melbourne: Victorian School for Deaf Children.

Johnston, S., \& Cosbey, J. (2012). Building blocks of a beginning communication system: Communicative modes. In S. Johnston, J. Reichle, K. Feeley \& E. Jones (Eds.), AAC strategies for individuals with moderate to severe disabilities (pp. 25-50). Baltimore: Paul H. Brookes Publishing Co.

Johnston, S., \& Feeley, K. (2012). AAC system features. In S. Johnston, J. Reichle, K. Feeley \& E. Jones (Eds.), AAC strategies for individuals with moderate to severe disabilities (pp. 51-80). Baltimore: Paul H. Brookes Publishing Co.

Kagohara, D., van der Meer, L., Achmadi, D., Green, V., O'Reilly, M., Lancioni, G., . . Sigafoos, J. (2012). Teaching picture naming to two adolescents with autism spectrum disorders using systematic instruction and speechgenerating devices. Research in Autism Spectrum Disorders, 6, 12241233.

Kagohara, D., van der Meer, L., Achmadi, D., Green, V., O'Reilly, M., Mulloy, A., ... Sigafoos, J. (2010). Behavioral intervention promotes successful use of an i-Pod-based communication device by an adolescent with autism Clinical Case Studies, 9, 328-338.

Kanner, L. (1943). Autistic disturbances of affective contact. Nervous Child, 2 , 217-250.

Kennedy, C. (2005). Single-case designs for educational research. Boston: Pearson Education Inc.

Kennedy, G. (Ed.). (1997). A dictionary of New Zealand sign language. Auckland: Auckland University Press: Bridget Williams Books.

Koegel, R., \& Koegel, L. (2006). Pivotal response treatments for autism. Baltimore: Paul H. Brookes Publishing.

Koul, R., Schlosser, R., \& Sancibrian, S. (2001). Effects of symbol, referent and instructional variables on the acquisition of aided and unaided symbols by individuals with autism spectrum disorders. Focus on Autism \& Other Developmental Disabilities, 16, 162-169. 
Lancioni, G., O'Reilly, M., Cuvo, A., Singh, N., Sigafoos, J., \& Didden, R. (2007). PECS and VOCAs to enable students with developmental disabilities to make requests: An overview of the literature. Research in Developmental Disabilities, 28, 468-488.

Laraway, S., Snycerski, S., Michael, J., \& Poling, A. (2003). Motivating operations and terms to describe them: Some further refinements. Journal of Applied Behavior Analysis, 36, 407-414.

Light, J., Roberts, B., Dimarco, R., \& Greiner, N. (1998). Augmentative and alternative communication to support receptive and expressive communication for people with autism. Journal of Communication Disorders, 31, 153-180.

Linscheid, T. (1999). Commentary: Response to empirically supported treatments for feeding problems. Journal of Pediatric Psychology, 24, 215-216.

Lloyd, L., Fuller, D., \& Arvidson, H. (1997). Augmentative and alternative communication: A handbook of principles and practices. Boston: Allyn \& Bacon.

Losh, M., Sullivan, P., Trembath, D., \& Piven, J. (2008). Current developments in the genetics of autism: From phenome to genome. Journal of Neuropathology and Experimental Neurology, 67, 829-837.

Lovaas, O. (1977). The autistic child: Language development through behavior modification. New York: Irvington.

Lovaas, O. (1987). Behavioral treatment and normal educational and intellectual functioning in young autistic children. Journal of Consulting and Clinical Psychology, 55, 3-9.

Lovaas, O. (2003). Teaching individuals with developmental delays: Basic intervention techniques. Austin, TX: Pro-Ed.

Lovaas, O., Simmons, J., Koegel, R., \& Stevens-Long, J. (1973). Some generalization and follow-up measures on autistic children in behavior therapy. Journal of Applied Behavior Analysis, 6, 131-166.

Makaton New Zealand/Aotearoa. (1998-99). Sign illustrations for Makaton core vocabulary. Auckland: Westprint. 
Mancil, G. (2009). Milieu therapy as a communication intervention: A review of the literature related to children with autism. Education and Training in Developmental Disabilities, 44, 105-117.

Mandell, D., Stahmer, A., \& Brodkin, E. (2008). Autism spectrum disorders in childhood. In T. Gullotta \& G. Blau (Eds.), Handbook of childhood behavioral issues: Evidence-based approaches to prevention and treatment (pp. 345-369). New York: Routledge Taylor \& Francis Group.

Matson, J., Benavidez, D., Compton, L., Paclawskyj, T., \& Baglio, C. (1996). Behavioral treatment of autistic persons: A review of research from 1980 to the present. Research in Developmental Disabilities, 17, 433-465.

Matson, J., \& Kozlowski, A. (2011). The increasing prevalence of autism spectrum disorders. Research in Autism Spectrum Disorders, 5, 418-425.

Matson, J., Kozlowski, A., \& Matson, M. (2012). Speech deficits in persons with autism: Etiology and symptom presentation. Research in Autism Spectrum Disorders, 6, 573-577.

Matson, J., Nebel-Schwalm, M., \& Matson, M. (2007). A review of methodological issues in the differential diagnosis of autism spectrum disorders in children. Research in Autism Spectrum Disorders, 1, 38-54.

Mayer-Johnson Co. (1994). Picture communication symbols combination book. Solano Beach, CA: Author.

McEachin, J., Smith, T., \& Lovaas, O. (1993). Long-term outcome for children with autism who received early intensive behavioral treatment. American Journal of Mental Retardation, 97, 359-372.

Michael, J. (1985). Two kinds of verbal behavior plus a possible third. The Analysis of Verbal Behavior, 3, 1-4.

Michael, J. (1988). Establishing operations and the mand. The Analysis of Verbal Behavior, 6, 3-9.

Ministries of Health and Education. (2008). New Zealand autism spectrum disorder guideline. Wellington: Author.

Mirenda, P. (1997). Supporting individuals with challenging behavior through functional communication training and AAC: Research review. AAC: Augmentative \& Alternative Communication, 13, 207-225. 
Mirenda, P. (2001). Autism, augmentative communication, and assistive technology: What do we really know? . Focus on Autism \& Other Developmental Disabilities, 16, 141-151.

Mirenda, P. (2003). Toward functional augmentative and alternative communication for students with autism: Manual signs, graphic symbols, and voice output communication aids. Language, Speech, \& Hearing Services in Schools, 34, 203-216.

Mirenda, P. (2008). A back door approach to autism and AAC. AAC: Augmentative \& Alternative Communication, 24, 220-234.

Mirenda, P. (2009). Promising innovations in AAC for individuals with autism spectrum disorders. Perspectives on Augmentative and Alternative Communication, 18, 112-113.

Mirenda, P., \& Erickson, K. (2000). Augmentative communication and literacy. In A. Wetherby \& B. Prizant (Eds.), Autism spectrum disorders: A transactional developmental perspective (pp. 333-367). Baltimore: Paul $\mathrm{H}$. Brookes.

Mustonen, T., Locke, P., Reichle, J., Solbrack, M., \& Lindgren, A. (1991). An overview of augmentative and alternative communication systems. In J. Reichle, J. York \& J. Sigafoos (Eds.), Implementing augmentative and alternative communication: Strategies for learners with severe disabilities (pp. 1-38). Baltimore: Paul H. Brookes Publishing Co.

National Joint Committee for the Communicative Needs of Persons with Severe Disabilities. (1992). Guidelines for meeting the communicative needs of persons with severe disabilities. American Speech-Language-Hearing Association, 34, 1-8.

National Research Council. (2001). Educating children with autism. Washington, DC: Henry (Joseph) Press.

Nederlands Gebarencentrum. (2006). Gebarenwoordenboek vor kinderen 1. Bunnik: Author.

Nunes, D. (2008). AAC interventions for autism: A research summary. International Journal of Special Education, 23, 17-26. 
Odom, S., Boyd, B., Hall, L., \& Hume, K. (2010). Evaluation of comprehensive treatment models for individuals with autism spectrum disorders. Journal of Autism and Developmental Disorders, 40, 425-436.

Odom, S., Brown, W., Frey, T., Karasu, N., Smith-Canter, L., \& Strain, P. (2003). Evidence-based practices for young children with autism: Contributions for single-subject design research. Focus on Autism \& Other Developmental Disabilities, 18, 166-174.

Odom, S., Horner, R., Snell, M., \& Blacher, J. (2007). The construct of developmental disbilities. In S. Odom, R. Horner, M. Snell \& J. Blacher (Eds.), Handbook of developmental disabilities (pp. 3-14). New York: The Guilford Press.

Ogletree, B., \& Harn, W. (2001). Augmentative and alternative communication for persons with autism: History, issues and unanswered questions. Focus on Autism \& Other Developmental Disabilities, 16, 138-140.

Pace, G., Ivancic, M., Edwards, G., Iwata, B., \& Page, T. (1985). Assessment of stimulus preference and reinforcer value with profoundly retarded individuals. Journal of Applied Behavior Analysis, 18, 249-255.

Parker, R., \& Vannest, K. (2009). An improved effect size for single-case research: Nonoverlap of all pairs. Behavior Therapy, 40, 357-367.

Passerino, L., \& Santarosa, L. (2008). Autism and digital learning environments: Processes of interaction and mediation. Computers and Education, 51, 385-402.

Paul, R. (1987). Communication. In D. Cohen \& A. Donnellan (Eds.), Handbook of autism and pervasive developmental disorders (pp. 61-84). New York: Wiley \& Sons.

Paul, R. (2008). Interventions to improve communication in autism. Child and Adolescent Psychiatric Clinics of North America, 17, 835-856.

Peeters, T., \& Gillberg, C. (1999). Autism: Medical and educational aspects. London: Whurr.

Piazza, C., Fisher, W., Hagopian, L., Bowman, L., \& Toole, L. (1996). Using a choice assessment to predict reinforcer effectiveness. Journal of Applied Behavior Analysis, 29, 1-9. 
Potter, B., \& Brown, D. (1997). A review of studies examining the nature of selection-based and topography-based verbal behavior. The Analysis of Verbal Behavior, 14, 85-104.

Poulson, C. (2009). Behavioral theory and language acquisition. In A. Fitzer \& P. Sturmey (Eds.), Language and autism: Applied behavior analysis, evidence, and practice (pp. 51-77). Austin, TX: Pro-Ed.

Preston, D., \& Carter, M. (2009). A review of the efficacy of the Picture Exchange Communication System intervention. Journal of Autism and Developmental Disorders, 39, 1471-1486.

Prizant, B., \& Schuler, A. (1987). Facilitating communication: Theoretical foundations. In D. Cohen \& A. Donnellan (Eds.), Handbook of autism and pervasive developmental disorders (pp. 289-300). New York: Wiley \& Sons.

Prizant, B., \& Wetherby, A. (2005). Critical issues in enhancing communication abilities for persons with autism spectrum disorders. In F. Volkmar (Ed.), Handbook of autism and pervasive developmental disorders (3rd ed., pp. 925-945). Hoboken, NJ: John Wiley \& Sons.

Pyramid Educational Products Inc. (2009). PICS for PECS 2009. Newport: Author.

Rehfeldt, R., Ziomek, M., \& Garcia, Y. (2006). Reinforcement contingencies in the mand and tact relations. European Journal of Behavior Analysis, 7, 125128.

Reichle, J. (1991). Defining the decisions involved in designing and implementing augmentative and alternative communication systems. In J. Reichle, J. York \& J. Sigafoos (Eds.), Implementing augmentative and alternative communication: Strategies for learners with severe disabilities (pp. 39-60). Baltimore: Paul H. Brookes Publishing Co.

Reichle, J., Beukelman, D., \& Light, J. (2002). Exemplary practices for beginning communicators: Implications for AAC. Baltimore: Paul $\mathrm{H}$. Brookes Publishing Co.

Reichle, J., \& Brady, N. (2012). Teaching pragmatic skills to individuals with severe disabilities. In S. Johnston, J. Reichle, K. Feeley \& E. Jones (Eds.), 
AAC strategies for individuals with moderate to severe disabilities (pp. 123). Baltimore: Paul H. Brookes Publishing Co.

Reichle, J., \& Ward, M. (1985). Teaching discriminative use of an encoding electronic communication device and signing exact English to a moderately handicapped child. Language, Speech, and Hearing Services in Schools, 16, 58-63.

Reid, D., \& Green, C. (2006). Preference-based teaching: Helping students with severe disabilities enjoy learning without problem behavior. TEACHING Exceptional Children Plus, 2. Retrieved from http://escholarship.bc.edu/education/tecplus/vol2/iss3/art2

Reid, D., O'Kane, N., \& Macurik, K. (2011). Staff training and management. In W. Fisher, C. Piazza \& H. Roane (Eds.), Handbook of applied behavior analysis (pp. 281-294). New York: Guilford Press.

Ringdahl, J., Falcomata, T., Christensen, T., Bass-Ringdahl, S., Lentz, A., Dutt, A., \& Schuh-Claus, J. (2009). Evaluation of a pre-treatment assessment to select mand topographies for functional communication training. Research in Developmental Disabilities, 20, 330-341.

Rispoli, M., Franco, J., van der Meer, L., Lang, R., \& Carmargo, S. (2010). The use of speech generating devices in communication interventions for individuals with developmental disabilities: A review of the literature. Developmental Neurorehabilitation, 13, 276-293.

Ritvo, E., Freeman, B., Pingree, C., Mason-Brothers, A., Jorde, L., Jenson, W., . . Ritvo, A. (1989). The UCLA-University of Utah epidemiologic survey of autism: Prevalence. The American Journal of Psychiatry, 146, 194-199.

Rotholz, D., Berkowitz, S., \& Burberry, J. (1989). Functionality of two modes of communication in the community by students with developmental disabilities: A comparison of signing and communication books. Journal of the Association for Persons with Severe Handicaps, 14, 227-233.

Rowland, C. (2009). Presymbolic communicators with autism spectrum disorders In P. Mirenda \& T. lacono (Eds.), Autism spectrum disorders and AAC (pp. 51-81). Baltimore: Paul H Brookes Publishing Co. 
Sailor, W., Gee, K., Goetz, L., \& Graham, N. (1988). Progress in educating students with the most severe disabilities: Is there any? Journal of The Association for Persons with Severe Handicaps, 13, 97-99.

Schepis, M., Reid, D., Behrmann, M., \& Sutton, K. (1998). Increasing communicative interactions of young children with autism using a voice output communication aid and naturalistic teaching. Journal of Applied Behavior Analysis, 31, 561-578.

Schlosser, R. (2003a). Roles of speech output in augmentative and alternative communication: Narrative review. AAC: Augmentative \& Alternative Communication, 19, 5-27.

Schlosser, R. (2003b). Comparative efficacy studies using single-subject experimental designs: How can they inform evidence-based practice? In R. Schlosser (Ed.), The efficacy of augmentative and alternative communication: Toward evidence-based practice (Vol. 553-595). San Diego: Academic Press.

Schlosser, R. (2003c). Single-subject designs. In R. Schlosser (Ed.), The efficacy of augmentative and alternative communication: Toward evidence-based practice (pp. 85-143). San Diego: Academic Press.

Schlosser, R. (2003d). The efficacy of augmentative and alternative communication: Toward evidence-based practice. San Diego: Academic Press.

Schlosser, R., \& Blischak, D. (2001). Is there a role for speech output in interventions for persons with autism? Focus on Autism \& Other Developmental Disabilities, 16, 170-178.

Schlosser, R., Blischak, D., \& Koul, R. (2003). Roles of speech-output in AAC: An integrative review. In R. Schlosser (Ed.), The efficacy of augmentative and alternative communication: Toward evidence-based practice (pp. 471532). San Diego: Academic Press.

Schlosser, R., \& Lee, D. (2000). Promoting generalization and maintenance in augmentative and alternative communication: A meta-analysis of 20 years of effectiveness research. AAC: Augmentative and Alternative Communication, 16, 208-226. 
Schlosser, R., \& Raghavendra, P. (2003). Toward evidence-based practice in AAC. In R. Schlosser (Ed.), The efficacy of augmentative and alternative communication: Toward evidence-based practice (pp. 260-297). San Diego: Academic Press.

Schlosser, R., \& Sigafoos, J. (2006). Augmentative and alternative communication interventions for persons with developmental disabilities: Narrative review of comparative single-subject experimental studies. Research in Developmental Disabilities, 27, 1-29.

Schlosser, R., \& Sigafoos, J. (2009). Navigating evidenced-based information sources in augmentative and alternative communication. $A A C$ : Augmentative and Alternative Communication, 25, 225-235.

Schlosser, R., Sigafoos, J., \& Koul, R. (2009). Speech output and speechgenerating devices in autism spectrum disorders. In P. Mirenda \& $\mathrm{T}$. lacono (Eds.), Autism spectrum disorders and AAC (pp. 141-170). Baltimore: Paul H. Brookes Publishing Co.

Schlosser, R., \& Wendt, O. (2008). Augmentative and alternative communication intervention for children with autism. In J. Luiselli, D. Russo, W. Christian \& S. Wilczynski (Eds.), Effective practices for children with autism: Educational and behavioral support interventions that work (pp. 325-389). Oxford: Oxford University Press.

Schuler, A., \& Baldwin, M. (1981). Nonspeech communication and childhood autism. Language, Speech, and Hearing Services in Schools, 12, 246-257.

Schuler, A., \& Prizant, B. (1987). Facilitating communication: Prelanguage approaches. In D. Cohen, A. Donnellan \& R. Paul (Eds.), Handbook of autism and pervasive developmental disorders (pp. 301-315). New York: Wiley \& Sons.

Seal, B., \& Bonvillian, J. (1997). Sign language and motor functioning in students with autistic disorder. Journal of Autism and Developmental Disorders, 27, 437-466.

Sennott, S., \& Bowker, A. (2009). Autism, AAC, and Proloquo2Go. Perspectives on Augmentative and Alternative Communication, 18, 137-145. 
Shafer, E. (1993). Teaching topography-based verbal behavior to developmentally disabled individuals: Some considerations. The Analysis of Verbal Behavior, 11, 117-133.

Shafer, E. (1994). A review of interventions to teach a mand repertoire. The Analysis of Verbal Behavior, 12, 53-66.

Shane, H., Laubscher, E., Schlosser, R., Flynn, S., Sorce, J., \& Abramson, J. (2011). Applying technology to visually support language and communication in individuals with autism spectrum disorders. Journal of Autism and Developmental Disorders. doi: 10.1007/s10803-011-1304-z

Shogren, K., Faggella-Luby, M., Bae, S., \& Wehmeyer, M. (2004). The effect of choice-making as an intervention for problem behavior: A meta-analysis. Journal of Positive Behavior Interventions, 6, 228-237.

Short, A., \& Schopler, E. (1988). Factors relating to age of onset in autism. Journal of Autism and Developmental Disorders, 18, 207-216.

Sidman, M. (1960). Tactics of scientific research: Evaluating experimental data in psychology. New York: Basic Books.

Sigafoos, J. (1998). Choice making and personal selection strategies. In J. Luiselli \& M. Cameron (Eds.), Antecedent control: Innovative approaches to behavioral support (pp. 187-221). Baltimore: Paul H. Brookes Publishing Co.

Sigafoos, J. (2006). Self-determination: Can we let the child determine the "best" treatment? Pediatric Rehabilitation, 9, 1-2.

Sigafoos, J., \& Drasgow, E. (2001). Conditional use of aided and unaided AAC: A review and clinical case demonstration. Focus on Autism \& Other Developmental Disabilities, 16, 152-161.

Sigafoos, J., Drasgow, E., Duker, P., O'Reilly, M., Lancioni, G., \& Reichle, J. (2009). General intervention approaches for teaching speech and its prerequisites. In A. Fitzer \& P. Sturmey (Eds.), Language and autism: Applied behavior analysis, evidence, and practice (pp. 109-133). Austin, TX: Pro-Ed.

Sigafoos, J., Drasgow, E., \& Schlosser, R. (2003). Strategies for beginning communicators. In R. Schlosser (Ed.), The efficacy of augmentative and 
alternative communication: Toward evidence-based practice (pp. 323346). San Diego: Academic Press.

Sigafoos, J., Ganz, J., O'Reilly, M., \& Lancioni, G. (2008). Evidence-based practice in the classroom: Evaluating a procedure for reducing perseverative requesting in an adolescent with autism and severe intellectual disability. Australasian Journal of Special Education, 32, 55-65.

Sigafoos, J., Green, V., Payne, D., Son, S., O'Reilly, M., \& Lancioni, G. (2009). A comparison of picture exchange and speech-generating deivces: Acquisition, pereference, and effects on social interaction. $A A C$ : Augmentative and Alternative Communication, 25, 99-109.

Sigafoos, J., \& lacono, T. (1993). Selecting augmentative communication devices for persons with severe disabilities: Some factors for educational teams to consider. Australian and New Zealand Journal of Developmental Disabilities, 18, 133-146.

Sigafoos, J., \& Mirenda, P. (2002). Strengthening communicative behaviors for gaining access to desired items and activities In J. Reichle, D. Beukelman \& J. Light (Eds.), Exemplary practices for beginning communicators: Implications for $A A C$ (pp. 123-256). Baltimore: Paul H. Brookes Publishing Co.

Sigafoos, J., O'Reilly, M., Ganz, J., Lancioni, G., \& Schlosser, R. (2005). Supporting self-determination in AAC interventions by assessing preference for communication devices. Technology and Disability, 17, 111.

Sigafoos, J., O'Reilly, M., Ganz, J., Lancioni, G., \& Schlosser, R. (2007). Assessing correspondence following acquisition of an exchange-based communication system. Research in Developmental Disabilities, 28, 7183.

Sigafoos, J., O'Reilly, M., \& Lancioni, G. (2009). Does the ASD label have validity? Developmental Neurorehabilitation, 12, 63-65.

Sigafoos, J., Roberts, D., Couzens, D., \& Kerr, M. (1993). Providing opportunities for choice-making and turn-taking to adults with multiple disabilities. Journal of Developmental and Physical Disabilities, 5, 297-310. 
Simpson, R., de Boer-Ott, S., Griswald, D., Myles, B., Byrd, S., Ganz, J. ... Adams, L. (2005). Autism spectrum disorders: Interventions and treatments for children and youth. Thousan Oaks, CA: Corwin Press.

Sindelar, P., Rosenberg, M., \& Wilson, R. (1985). An adapted alternating treatments design for instructional research. Education \& Treatment of Children, 8, 67-76.

Skinner, B. (1938). The behavior of organisms. New York: Appleton-CenturyCrofts.

Skinner, B. (1953). Science and human behavior. New York: Macmillan.

Skinner, B. (1957). Verbal Behavior. Englewood Cliffs, NJ: Prentice Hall.

Skinner, B. (1973). Self-control. In M. Goldfried \& M. Merbaum (Eds.), Behavior change through self-control (pp. 58-69). New York: Holt, Rinehart, and Winston, Inc.

Smith, T. (1996). Are other treatments effective? In C. Maurice (Ed.), Behavioral intervention for young children with autism (pp. 45-59). Austin, TX: Pro-Ed.

Son, S., Sigafoos, J., O'Reilly, M., \& Lancioni, G. (2006). Comparing two types of augmentative and alternative communication systems for children with autism. Pediatric Rehabilitation, 9, 389-395.

Soto, G., Belfiore, P., Schlosser, R., \& Haynes, C. (1993). Teaching specific requests: A comparative analysis of skill acquisition and preference using two augmentative and alternative communication aids. Education and Training in Mental Retardation, 28, 169-178.

Sparrow, S., Balla, D., \& Cicchetti, D. (2003). Vineland - Z Adaptive Behavior Scales (Dutch edition). Leiden: PITS.

Sparrow, S., Cicchetti, D., \& Balla, D. (2005). Vineland-II Adaptive Behavior Scales (2nd ed.). Minneapolis: Pearson.

Stafford, A., Alberto, P., Fredrick, L., Heflin, L., \& Heller, K. (2002). Preference variability and the instruction of choice making with students with severe intellectual disabilities. Education and Training in Mental Retardation and Developmental Disabilities, 37, 70-88.

Stancliffe, R. (2001). Living with support in the community: Predictors of choice and self-determination. Mental Retardation and Developmental Disabilities, 7, 91-98. 
Statistics New Zealand. (2007). Disability survey 2006. Retrieved 2 February, 2012, from

http://www.stats.govt.nz/browse_for_stats/health/disabilities/DisabilitySurv ey2006_HOTP06.aspx

Steyn, B., \& Couteur, A. (2003). Understanding autism spectrum disorders. Current Paediatrics, 13, 274-278.

Stromer, R., Kimball, J., Kinney, E., \& Taylor, B. (2006). Activity schedules, computer technology, and teaching children with autism spectrum disorders. Focus on Autism \& Other Developmental Disabilities, 21, 14-24.

Sulzer-Azaroff, B., Hoffman, A., Horton, C., Bondy, A., \& Frost, L. (2009). The Picture Exchange Communication System (PECS): What do the data say? Focus on Autism \& Other Developmental Disabilities, 24, 89-103.

Sundberg, C., \& Sundberg, M. (1990). Comparing topography-based verbal behavior with stimulus selection-based verbal behavior. The Analysis of Verbal Behavior, 8, 31-41.

Sundberg, M. (1993). Selecting a response form for nonverbal persons: Facilitated communication, pointing systems, or sign language? The Analysis of Verbal Behavior, 11, 99-116.

Thunberg, G., Ahlsen, E., \& Sandberg, A. (2007). Children with autistic spectrum disorders and speech-generating devices: Communication in different activities at home. Clinical Linguistics \& Phonetics, 21, 457-479.

Thunberg, G., Ahlsén, E., \& Sandberg, A. (2009). Interaction and use of speechgenerating devices in the homes of children with autism spectrum disorders-An analysis of conversational topics. Journal of Special Education Technology, 24, 1-17.

Thunberg, G., Sandberg, A., \& Ahlsén, E. (2009). Speech-generating devices used at home by children with autism spectrum disorders: A preliminary assessment. Focus on Autism \& Other Developmental Disabilities, 24, 104-115.

Tien, K. (2008). Effectiveness of the Picture Exchange Communication System as a functional communication intervention for individuals with autism spectrum disorders: A practice-based research synthesis. Education and Training in Developmental Disabilities, 43, 67-76. 
Tincani, M. (2004). Comparing the Picture Exchange Communication System and sign language training for children with autism. Focus on Autism \& Other Developmental Disabilities, 19, 152-163.

van der Meer, L., Didden, R., Sutherland, D., O'Reilly, M., Lancioni, G., \& Sigafoos, J. (2012). Comparing three augmentative and alternative communication modes for children with developmental disabilities. Journal of Developmental and Physical Disabilities, 24, 451-468.

van der Meer, L., Kagohara, D., Achmadi, D., Green, V., Herrington, C., Sigafoos, J., . . Rispoli, M. (2011). Teaching functional use of an iPod-based speech-generating device to students with developmental disabilities. Journal of Special Education Technology, 26, 1-12.

van der Meer, L., Kagohara, D., Achmadi, D., O'Reilly, M., Lancioni, G., Sutherland, D., \& Sigafoos, J. (2012). Speech-generating devices versus manual signing for children with developmental disabilities. Research in Developmental Disabilities, 33, 1658-1669.

van der Meer, L., \& Rispoli, M. (2010). Communication interventions involving speech-generating devices for children with autism: A review of the literature. Developmental Neurorehabilitation, 13, 294-306.

van der Meer, L., Sigafoos, J., O'Reilly, M., \& Lancioni, G. (2011). Assessing preferences for AAC options in communication interventions for individuals with developmental disabilities: A review of the literature. Research in Developmental Disabilities, 32, 1422-1431.

van der Meer, L., Sutherland, D., O'Reilly, M., Lancioni, G., \& Sigafoos, J. (2012). A further comparison of manual signing, picture exchange, and speechgenerating devices as communication modes for children with autism spectrum disorders. Research in Autism Spectrum Disorders.

Verschuur, R., Didden, R., Van der Meer, L., Achmadi, D., Kagohara, D., Green, V., . . Lancioni, G. (2011). Investigating the validity of a structured interview protocol for assessing the preferences of children with autism spectrum disorders. Developmental Neurorehabilitation, 14, 366-371.

Watson, L., \& Workman, E. (1981). The non-concurrent multiple baseline acrossindividuals design: An extension of the traditional multiple baseline design. Journal of Behavior Therapy and Experimental Psychiatry, 12, 257-259. 
Wehmeyer, M. (1992). Self-determination in the education of students with mental retardation. Education and Training in Mental Retardation, 27, 302314.

Wehmeyer, M., Palmer, S., Agran, M., Mithaug, D., \& Martin, J. (2000). Promoting causal agency: The self-determined learning model of instruction. Exceptional Children, 66, 439-453.

Wehmeyer, M., Sands, D., Doll, B., \& Palmer, S. (1997). The development of self-determination and implications for educational interventions with students with disabilities. International Journal of Disability, Development and Education, 44, 305-328.

Wehmeyer, M., \& Shogren, K. (2008). Self-determination and learners with autism spectrum disorders. In R. Simpson \& B. Myles (Eds.), Educating children and youth with autism: Strategies for effective practice (2nd ed., pp. 433-476). Austin, TX: Pro-Ed.

Wehmeyer, M., Shogren, K., Zager, D., Smith, T., \& Simpson, R. (2010). Research-based principles and practices for educating students with autism: Self-determination and social interactions. Education \& Training in Autism and Developmental Dlsabilities, 45, 475-486.

Weitz, C., Dexter, M., \& Moore, J. (1997). AAC and children with developmental disabilities. In S. Glennen \& D. DeCoste (Eds.), Handbook of augmentative and alternative communication (pp. 395-431). San Diego: Singular.

Wendt, O. (2007). The effectiveness of augmentative and alternative communication for individuals with autism spectrum disorders: A systematic review and meta-analysis. Doctoral dissertation, Purdue University, 2006. Dissertation Abstracts International: Section A: Humanities and Social Sciences, 68.

Wendt, O. (2009). Research on the use of manual signs and graphic symbols in autism spectrum disorders: A systematic review. In P. Mirenda \& T. lacono (Eds.), Autism spectrum disorders and AAC (pp. 83-140). Baltimore: Paul H. Brookes Publishing Co. 
Whitehouse, J., Barry, J., \& Bishop, D. (2008). Further defining the language impairment in autism: Is there a specific language impairment subtype? Journal of Communication Disorders, 41, 319-336.

Wilkinson, K. (1998). Profiles of language and communication skills in autism. Mental Retardation and Developmental Disabilities Research Reviews, 4, 73-79.

Wilkinson, K., \& Hennig, S. (2007). The state of research and practice in augmentative and alternative communication for children with developmental/intellectual disabilities. Mental Retardation and Developmental Disabilities, 13, 58-69.

Wilkinson, K., \& Mcllvane, W. (2002). Considerations in teaching graphic symbols to beginning communicators. In J. Reichle, D. Beukelman \& J. Light (Eds.), Examplary practices for beginning communicators: Implications for $A A C$ (pp. 273-321). Baltimore: Paul H Brookes Publishing Co.

Williams, J., Higgins, J., \& Brayne, C. (2006). Systematic review of prevalence studies of autism spectrum disorders. Archives of Disease in Childhood, 91, 8-15.

Winborn-Kemmerer, L., Ringdahl, J., Wacker, D., \& Kitsukawa, K. (2009). A demonstration of individual preference for novel mands during functional communication training. Journal of Applied Behavior Analysis, 42, 185189.

Wing, L. (1971). Autistic children. London: Constable and Co Ltd.

Wing, L. (1981). Asperger's syndrome: A clinical account. Psychological Medicine, 11, 115-130.

Wing, L., \& Attwood, A. (1987). Syndromes of autism and atypical development. In D. Cohen \& A. Donnellan (Eds.), Handbook of autism and pervasive developmental disorders (pp. 3-19). New York: Wiley \& Sons.

Wing, L., Yeates, S., Brierley, L., \& Gould, J. (1976). The prevalence of early childhood autism: Comparison of administrative and epidemiological studies. Psychological Medicine, 6, 89-100.

World Health Organization. (1992). International statistical classification of diseases and related health problems: ICD-10. Geneva, Switzerland: Author. 
Worley, J., \& Matson, J. (2012). Comparing symptoms of autism spectrum disorder using the current $D S M-I V-T R$ diagnostic criteria and the proposed $D S M-V$ diagnostic criteria. Research in Autism Spectrum Disorders, 6 , 965-970.

Wraikat, R., Sundberg, C., \& Michael, J. (1991). Topography-based and selection-based verbal behavior: A further comparison. The Analysis of Verbal Behavior, 9, 1-17.

Yamamoto, J., \& Mochizuki, A. (1988). Acquisition and functional analysis of manding with autistic students. Journal of Applied Behavior Analysis, 21, 57-64. 


\section{APPENDIX A}

Ethics approval letters for each of the three studies 
$5^{\text {th }}$ February 2010

Jeff Sigafoos and Vanessa Green

Professor and Associate Professor

Victoria University of Wellington Faculty of Education

$\mathrm{C} /$ - School of Educational Psychology and Pedagogy

Donald Street

Wellington

Dear Jeff and Vanessa

\section{RE: Ethics application SEPP/2009/43: RM 16778}

I am pleased to advise you that your ethics application 'Addendum to: Enhancing the communication, leisure and social skills of children with developmental disabilities', has been approved by the Victoria University of Wellington Faculty of Education Ethics Committee. Please note that the approval for your research to commence is from the date of this letter.

Good luck with your research.

Yours Sincerely

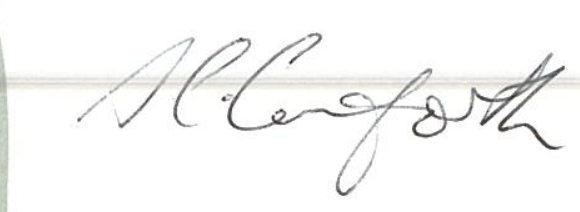

Dr Sue Cornforth

Co-Convener

Victoria University of Wellington Faculty of Education Ethics Committee 
19 July 2010

Larah van der Meer

PhD Student

Victoria University of Wellington Faculty of Education

$\mathrm{C} /$ - School of Educational Psychology and Pedagogy

Donald Street

Wellington

Dear Larah

\section{RE: Ethics application SEPP/2010/56: RM 17816}

I am pleased to advise you that your ethics application 'Comparing SpeechGenerating Devices and Manual Signing as Communication Aids for Children with Autism', with requested amendments, has been approved by the Victoria University of Wellington Faculty of Education Ethics Committee. Please note that the approval for your research to commence is from the date of this letter.

Best wishes for your research.

Yours Sincerely

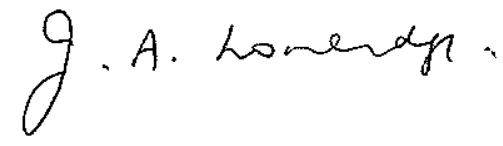

Dr Judith Loveridge

Co-Convener

Victoria University of Wellington Faculty of Education Ethics Committee 
$19^{\text {th }}$ November 2010

Jeff Sigafoos

Victoria University of Wellington Faculty of Education

$\mathrm{C} /$ - School of Educational Psychology and Pedagogy

Donald Street

Wellington

Dear Jeff

\section{RE: Ethics application SEPP/2010/92 RM 18095}

I am pleased to advise you that your ethics application 'Enhancing communication Intervention for Children with Autism', with the required changes, has been approved by the Victoria University of Wellington Faculty of Education Ethics Committee. Please note that the approval for your research to commence is from the date of this letter.

Best wishes for your research.

Yours Sincerely

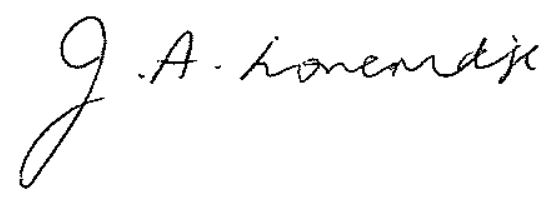

Dr Judith Loveridge

Co-Convener

Victoria University of Wellington Faculty of Education Ethics Committee 
Jeff Sigafoos

Victoria University of Wellington Faculty of Education

$\mathrm{C} /-$ School of Educational Psychology and Pedagogy

Donald Street

Wellington

Dear Jeff

RE: Ethics application SEPP/2011/18 Addendum to: SEPP/2010/92 RM $\underline{18095}$

I am pleased to advise you that your ethics application Addendum to: 'Enhancing communication Intervention for Children with Autism', with the required changes, has been approved by the Victoria University of Wellington Faculty of Education Ethics Committee.

Best wishes for your research.

Yours Sincerely

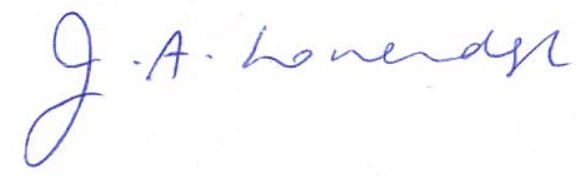

Dr Judith Loveridge

Co-Convener

Victoria University of Wellington Faculty of Education Ethics Committee 
Jeff Sigafoos

Victoria University of Wellington Faculty of Education

$\mathrm{Cl}$ - School of Educational Psychology and Pedagogy

Donald Street

Wellington

Dear Jeff

RE: Ethics application SEPP/2011/48 Addendum(2) to: SEPP/2010/92

\section{RM 18095}

I am pleased to advise you that your ethics application Addendum (2) to:

'Enhancing communication Intervention for Children with Autism', with the required changes, has been approved by the Victoria University of Wellington Faculty of Education Ethics Committee.

Best wishes for your research.

Yours Sincerely

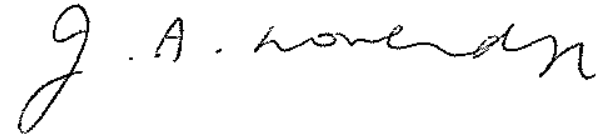

Dr Judith Loveridge

Co-Convener

Victoria University of Wellington Faculty of Education Ethics Committee 


\section{APPENDIX B}

van der Meer, L., \& Rispoli, M. (2010). Communication interventions involving speech-generating devices for children with autism: A review of the literature. Developmental Neurorehabilitation, 13, 294-306 


\title{
SUBJECT REVIEW
}

\section{Communication interventions involving speech-generating devices for children with autism: A review of the literature}

\author{
LARAH A. J. VAN DER MEER ${ }^{1} \&$ MANDY RISPOLI ${ }^{2}$ \\ ${ }^{1}$ Victoria University of Wellington, Wellington, New Zealand and ${ }^{2}$ Texas A E M University, College Station, TX, USA
}

(Received 1 February 2010; accepted 1 February 2010)

\begin{abstract}
Objective: The current review synthesizes communication intervention studies that involved the use of speech-generating devices (SGD) for children with autism.

Methods: Twenty-three studies were identified that met the inclusion criteria following systematic searches of electronic databases, journals and reference lists. Studies were evaluated in terms of: (a) participants, (b) setting, (c) mode of communication, (d) communication skill(s) taught to the participant, (e) intervention procedures, (f) outcomes, (g) follow-up and generalization, (h) reliability and treatment integrity and (i) design and certainty of evidence.

Results: Intervention, most commonly targeting requesting skills, was provided to a total of 51 children aged 3-16 years. Intervention strategies followed two main approaches: operant/behavioural techniques and naturalistic teaching procedures. Positive outcomes were reported for $86 \%$ of the studies and $78 \%$ of the studies were categorized as providing conclusive evidence.

Conclusion: The literature base suggests that SGDs are viable communication options for children with autism. However, several areas warrant future research.
\end{abstract}

Keywords: autism, ASD, speech generating device, voice-output communication aid, communication, intervention

\section{Resumen}

Objetivo: La actual revisión sintetiza los estudios sobre intervenciones de comunicación que utilizan dispositivos de generación del habla (SGD) para niños con autismo.

Métodos: Posterior a una búsqueda sistematizada de listas de referencias, revistas y bases de datos electrónicas se identificaron veintitrés estudios que cumplían con los criterios de inclusión. Los estudios fueron evaluados en términos de: (a) participantes, (b) escenario, (c) medio de comunicación, (d) habilidad(es) de comunicación enseñadas a los participantes, (e) procedimiento de intervención, (f) resultados, (g) seguimiento y generalización, (h) integridad y confiabilidad del tratamiento y (i) diseño y certeza de la evidencia.

Resultados: Se proporcionó intervención, con mayor interés en las habilidades de petición, a un total de 51 niños con edades comprendidas entre los 3 y los 16 años de edad. Las estrategias de intervención siguieron dos enfoques principalmente: técnicas operantes/conductuales y el enfoque de enseñanza naturalista. Se reportaron resultados positivos en el $86 \%$ de los estudios y el $78 \%$ de los estudios se categorizaron como aportadores de pruebas concluyente.

Conclusión: Esta literatura de base sugiere que los SGD son una opción viable de comunicación para niños con autismo. Sin embargo, múltiples áreas ameritan una mayor investigación.

Palabras clave: autismo, ASD, dispositivos de generación del habla, dispositivo de ayuda de comunicación de producción de la voz, comunicación, intervención

\section{Introduction}

Individuals with Autism Spectrum Disorders (ASD) are characterized by having significant impairments in social interaction, communication development and the presence of restricted, repetitive and stereotyped behaviour patterns [1]. Up to half of children with autism do not develop speech or develop only limited speech and language abilities [2-6]. Instead these children may rely on pre-linguistic behaviours, including pointing, 
reaching, eye-gazing and other facial expressions [7, 8]. Some children may also demonstrate challenging behaviour, such as aggression, tantrums and self-injury in an attempt to communicate their wants and needs [9, 10]. Such pre-linguistic behaviours become frustrating for both the communicator and the communication partner, as they are often difficult to interpret.

Romski et al. [11] explained that it is unclear whether children who lack speech at a young age will remain at this pre-linguistic level. Instead the child's communication status may change over time as a function of maturity, intervention or both. One intervention that these children may benefit from is augmentative and alternative communication (AAC). AAC refers to a practice that aims to supplement (i.e. augment) or replace (i.e. alternative) natural speech [12-15]. This is achieved either by unaided approaches, such as gestures or manual signing; or by aided systems, involving graphics (traditional orthography, photographs or line drawings). Aided systems use external equipment with a communicative function, such as Picture Exchange (PE) [16, 17] or Speech-Generating Devices (SGDs), otherwise referred to as voice output communication aids (VOCAs) [18].

In particular SGDs became prominent communication options for many individuals with autism by the 1980s and 1990s [19, 20]. A SGD is a portable electronic devise that will produce either digitized or synthesized speech output. The SGD displays a variety of graphic symbols to represent a message that is activated resulting in voice output when the individual uses a finger, hand or some other means to select the message [7].

To date SGD intervention research has typically involved teaching the individual to request access to highly preferred items $[18,21]$. For example, in a SGD intervention the individual might be taught to touch a picture or line drawing on the electronic speech output device, which produces a prerecorded message, such as 'I want _.' In return the communication partner will deliver the requested item [22]. In addition to requesting, a number of other communicative functions, such as commenting, greeting or answering questions would be important to teach to individuals with ASD [23]. Most of these communication skills have been taught within a positivist behaviour analytic approach using operant methods such as discrete trial training [24]. However, recently naturalistic approaches to teaching SGD use have been increasing [25].

Depending on communicative goals, a large variety of SGDs can be selected and customized for intervention. SGDs can vary in design including permanence of the display (static or dynamic), number of graphic representations on the display and size of the graphic symbols [26]. Consideration of the type of voice-output used (digitized vs synthesized) can also be important. For example, it has been hypothesized that the lack of variability and robotic nature of synthesized speech may need to be considered when implementing AAC with individuals with autism [27]. The voice-output feature of SGDs might also make this a more readily understood mode of communication, thus promoting greater community inclusion and participation [28].

An emerging corpus of intervention research has investigated the use of SGDs as well as AAC in general with individuals with developmental disabilities or autism and there are several reviews that have focused on a number of issues related to this topic $[7,18,19,27,29,30]$. Schlosser and Lee [29], for example, provide support for the use of AAC in general, but did not focus on individual modalities of AAC, such as SGDs. Lancioni et al. [18] focused on the use of SGDs and PECs in teaching requesting behaviours to individuals with developmental disabilities. They concluded that outcomes are encouraging, but methodological concerns provide reason for results to be interpreted with caution. Only Schlosser et al. [19] appear to specifically review research assessing the use of SGDs for individuals with autism. Thus, in order to answer empirical questions that remain in this field [31], a systematic review of SGD interventions with individuals with autism is warranted. The aim of the current report is to systematically review the literature in this field and thereby evaluate whether there is evidence to support that children with autism are capable of learning to use an SGD to communicate. Specific objectives are to provide an up-to-date synthesis of the literature in order to (a) assist clinicians in their practice of improving the communication of children with autism and (b) identify gaps in the literature and areas in need of further research. Together these objectives may help to guide and inform evidence-based practice with respect to the use of SGDs in communication interventions for children with autism.

\section{Method}

\section{Search procedures}

Systematic searches were conducted in six electronic databases: Cumulative Index of Nursing and Allied Health Literatures (CINAHL), Education Resources Information Center (ERIC), Medline, Linguistics and Language Behavior Abstracts (LLBA), Proquest and PsycINFO. Publication year was not restricted, but the search was limited to English-language journal articles. The search covered all dates covered by these databases up to September 2009 . 
For the CINAHL search, voice-output communication aid (or speech-generating device) and autism was entered into the All Text field. For the remaining databases, the free-text terms voice-output communication aid (or VOCA or speech-generating device) and autism (or autism spectrum disorders) were inserted into the Keywords field. Abstracts of the records returned from these electronic searches were reviewed to identify studies for inclusion in the review (see Inclusion and Exclusion Criteria).

Three additional search strategies were used in order to find other possibly relevant studies that may have been missed by the electronic search. First, the reference lists for the included studies were reviewed to identify additional articles for possible inclusion. Secondly, hand searches were completed for the journals that had published the included studies. Finally, using an author search, the five databases were searched again for additional related work by authors of the studies that met the inclusion criteria. From this combination of search procedures, 25 articles were identified for possible inclusion in the systematic review.

\section{Inclusion and exclusion criteria}

To be included in this review, the article had to be a research study that included children $(<18$ years of age) with ASD and examined the effects of an intervention involving SGDs. Intervention was defined as implementing one or more therapeutic/ teaching procedures for the purpose of trying to increase or improve the child's communication skills or abilities through the use of a SGD. Examples could include teaching a child to use an SGD to (a) make requests, (b) spell words or (c) repair a communicative breakdown.

The research study had to obtain empirical data from which one could assess the success of the intervention. For example, a paper by Light et al. [32] initially identified for inclusion used case reports that did not provide objective data on SGD use and was therefore not included in the current review. Studies that focused only on the description of or assessment of communication skills were not included.

\section{Data extraction}

Each study identified was first evaluated to establish if it met the pre-determined inclusion criteria. All studies that met the inclusion criteria were then coded in terms of: (a) participants (e.g. age, gender, number and diagnosis), (b) setting (e.g. school, home or community setting), (c) mode of communication (e.g. type of SGD used), (d) communication skill(s) taught to the participant, (e) intervention procedures (e.g. least to most prompting), (f) outcomes of the intervention, (g) follow-up and generalization, if any, (h) reliability and treatment integrity and (i) experimental design and certainty of evidence. The certainty of evidence was rated as either conclusive or inconclusive [33, 34] in order to provide an overview of the quality of the evidence across the studies reviewed [35].

\section{Inter-rater agreement}

The initial search of the six databases revealed 15 articles that met the inclusion criteria for this review. Three articles were identified in the reference list search; three articles were identified in the journal search and finally three articles were identified in the author search, resulting in a total of 24 articles for inclusion in this review. To assess inter-rater agreement, an independent rater reviewed these 24 articles according to the inclusion criteria. This resulted in $100 \%$ agreement for the initial three search methods. However, one discrepancy was identified in the author search. Upon review, this study [6] was excluded because the participant did not have a formal ASD diagnosis. Therefore, a total of 23 articles met the inclusion criteria.

\section{Results}

A total of 29 interventions/experiments were reported in the 23 included studies. Table I summarizes the purpose, participants, type of SGD, outcomes and design and certainty of evidence for each of the 23 included studies.

\section{Participants}

A total of 51 participants with ASD were included in the studies. When a study included participants with and without an ASD diagnosis, only data from the ASD participants were coded. Of these 51 participants, $90.2 \%(n=46)$ were boys and $9.8 \%$ $(n=5)$ were girls, a ratio of nine boys to every girl. The majority of participants were reported as having autism $(n=34,66.7 \%)$, five as having ASD $(9.8 \%)$ and $12(23.5 \%)$ with pervasive developmental disorder-not otherwise specified (PDD-NOS). Of those with autism or ASD, diagnosis ranged from mild-to-severe and included Autistic disorder. Thirty-seven per cent of participants $(n=19)$ also had a dual diagnosis of ASD and some level of intellectual disability or other developmental delay $[10,36-43]$. In one study [24] the sole participant had a diagnosis of Down syndrome and Autistic disorder.

Ages ranged from 3-16 years (mean $=7.7$ ). In one study [44] age was not specifically identified, instead it was stated that participants were pre-school aged. 

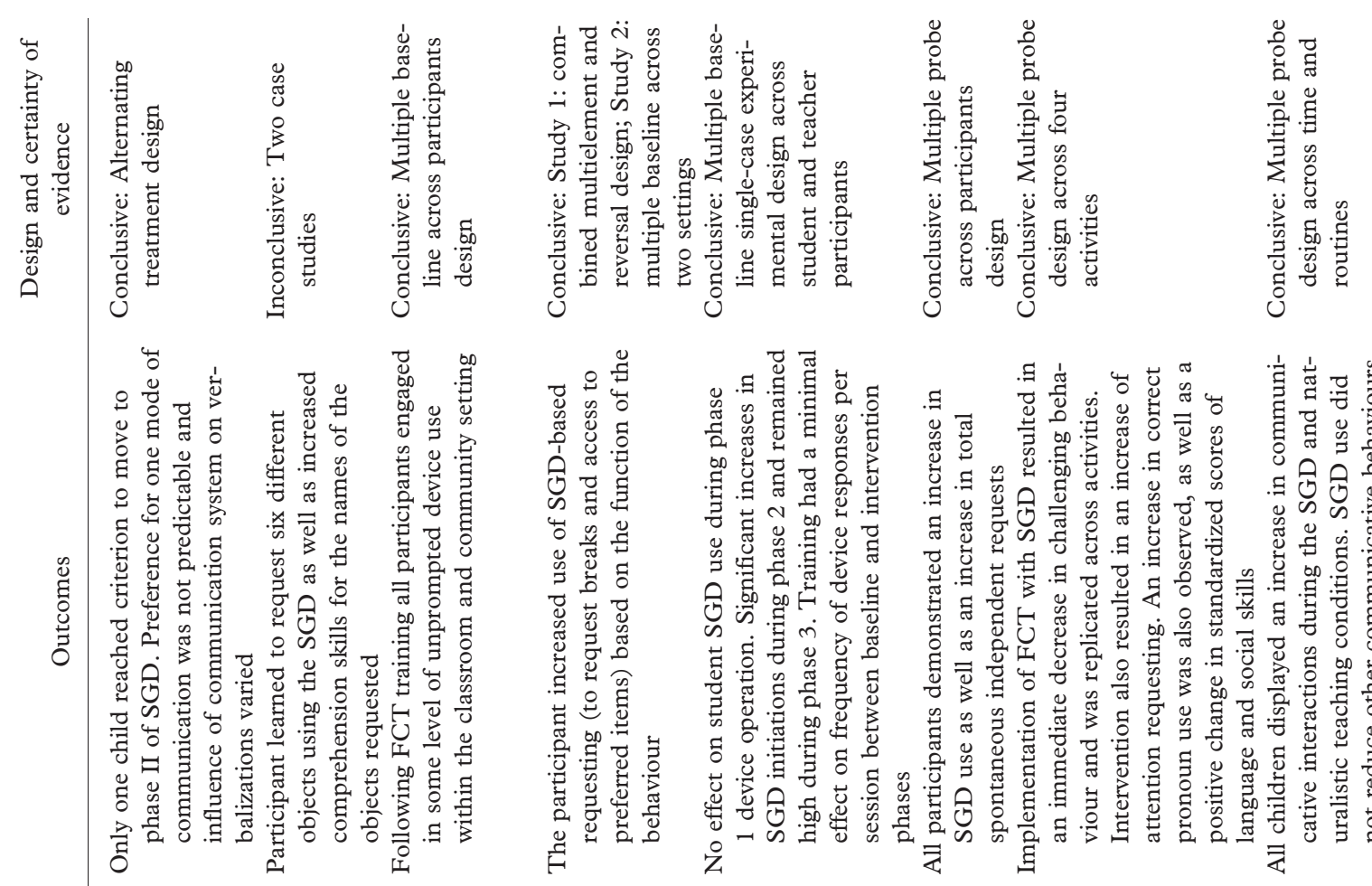

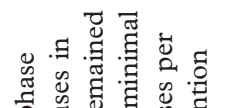

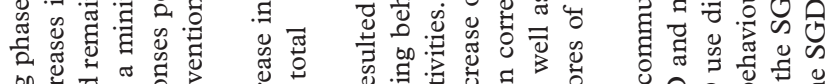

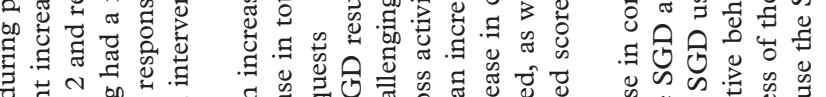

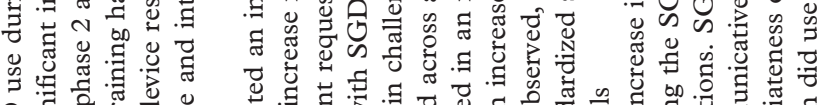

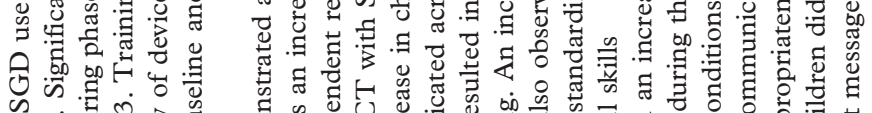

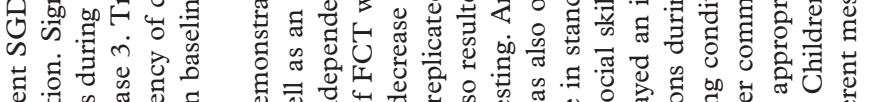

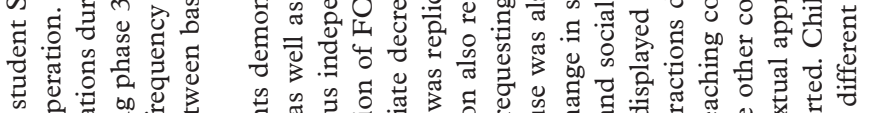

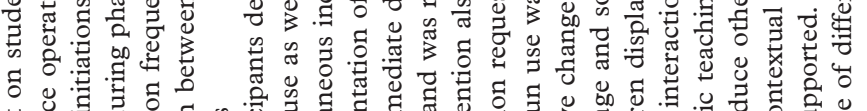

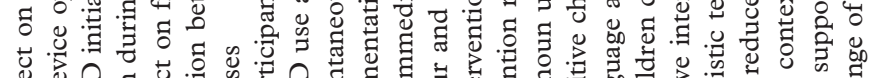

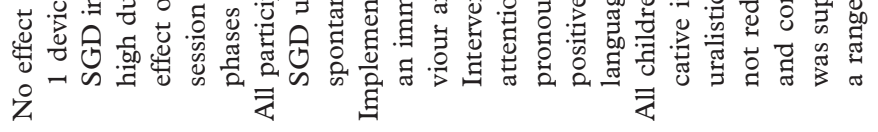

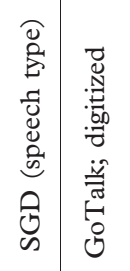
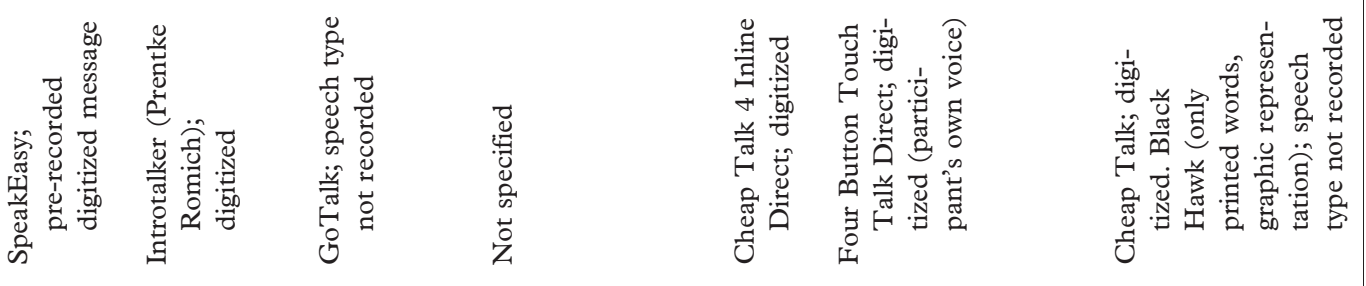

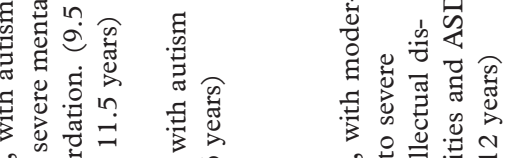
등ㅇㅇ 㲾

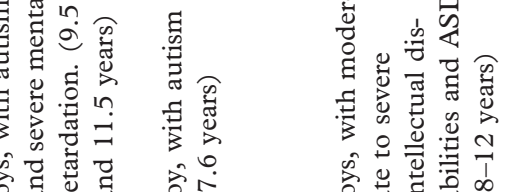

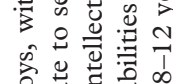

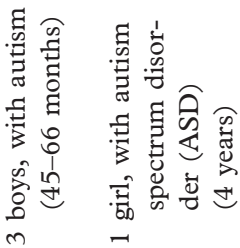

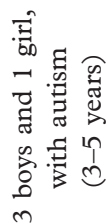

0
0
0
$\vdots$
$\vdots$

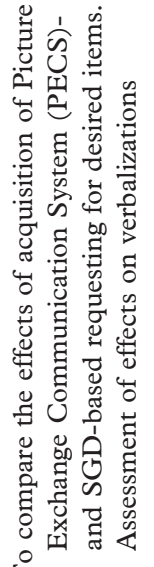

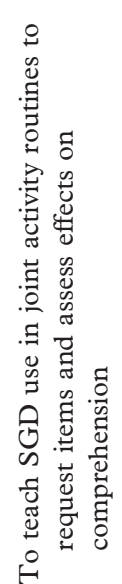
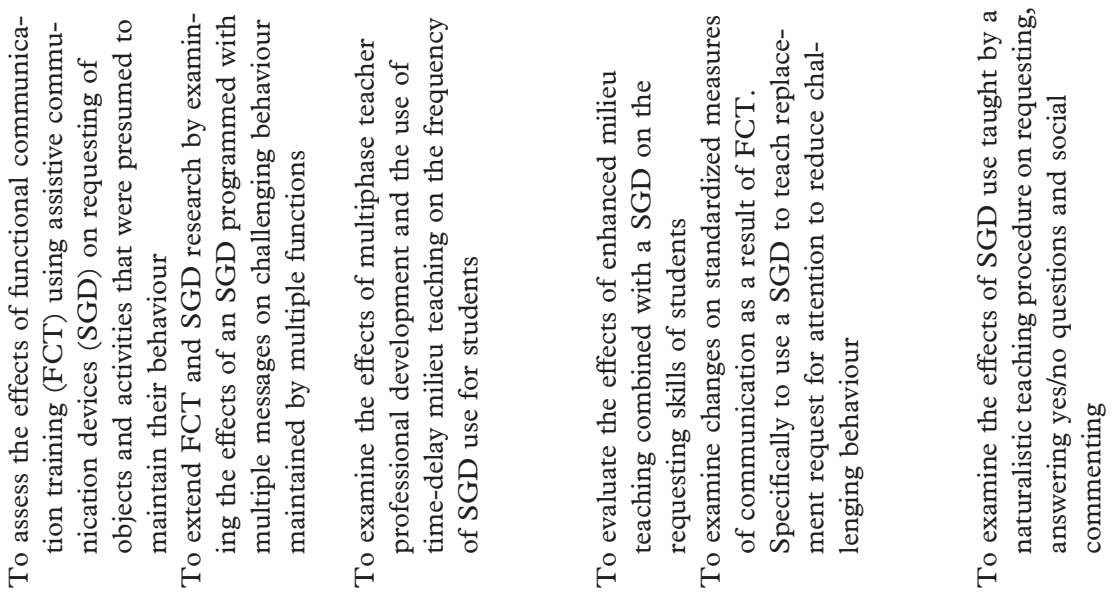

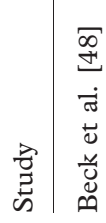

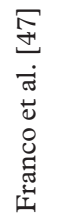

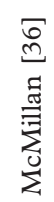

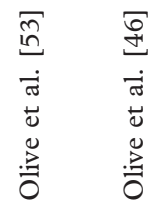

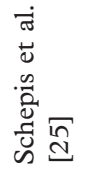




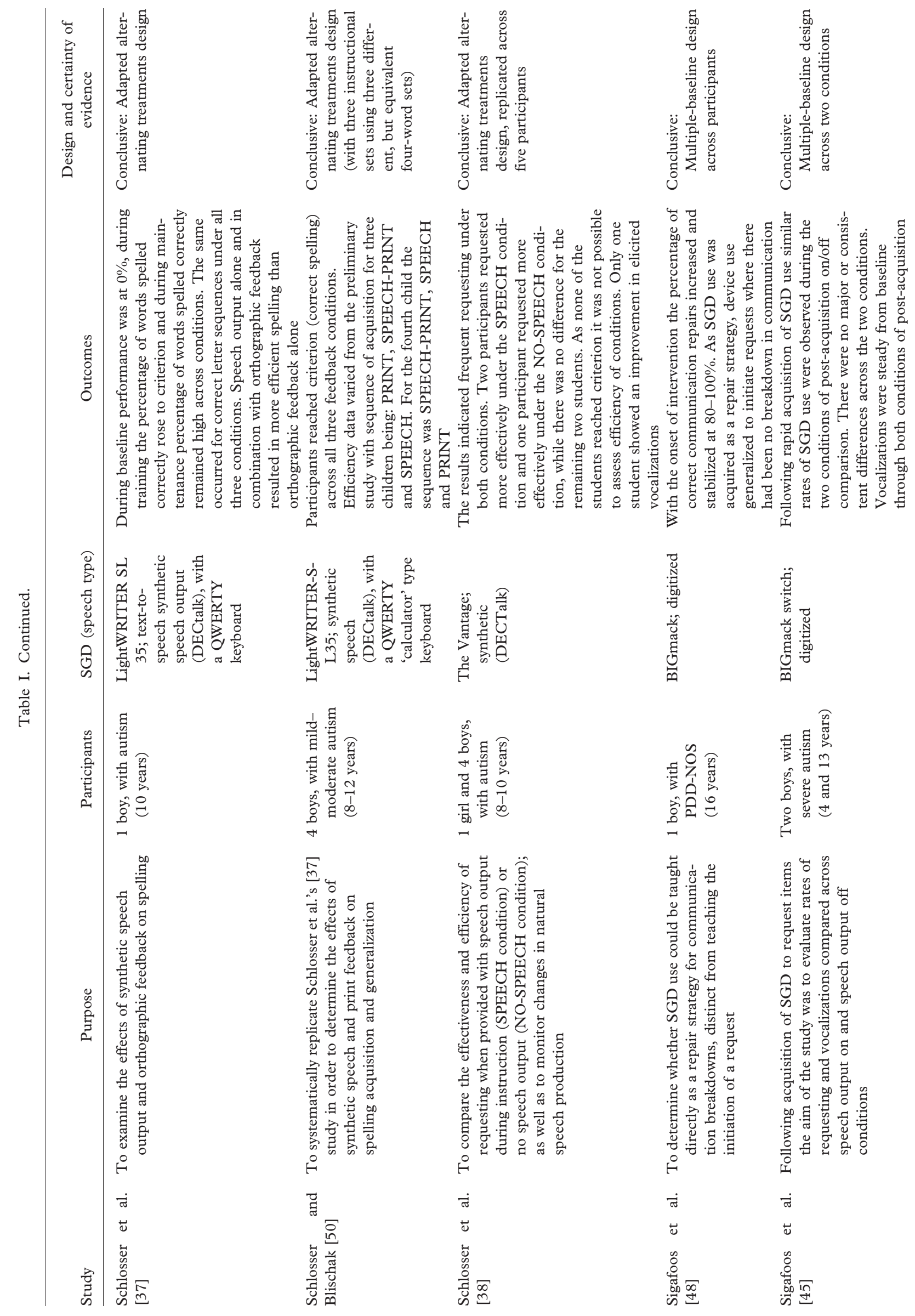




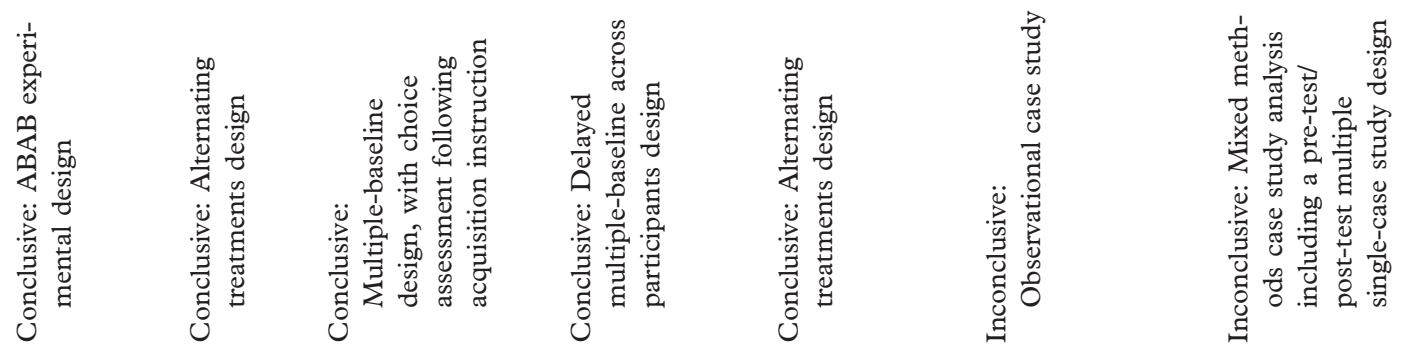

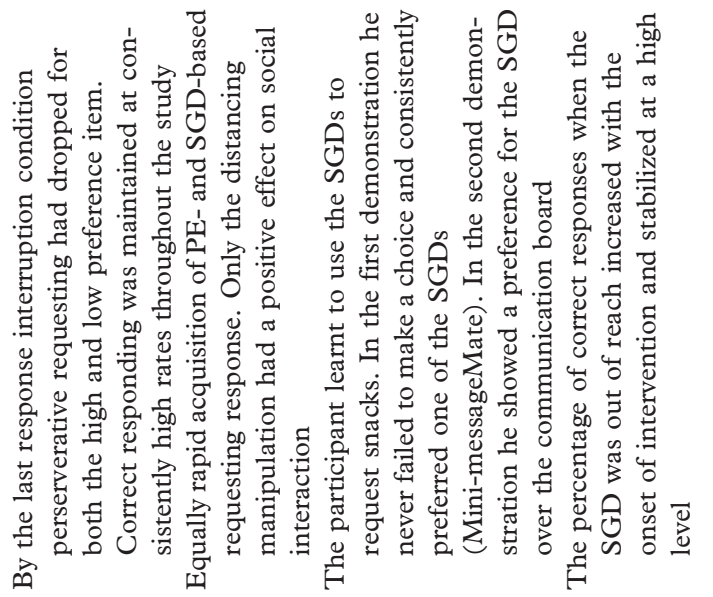
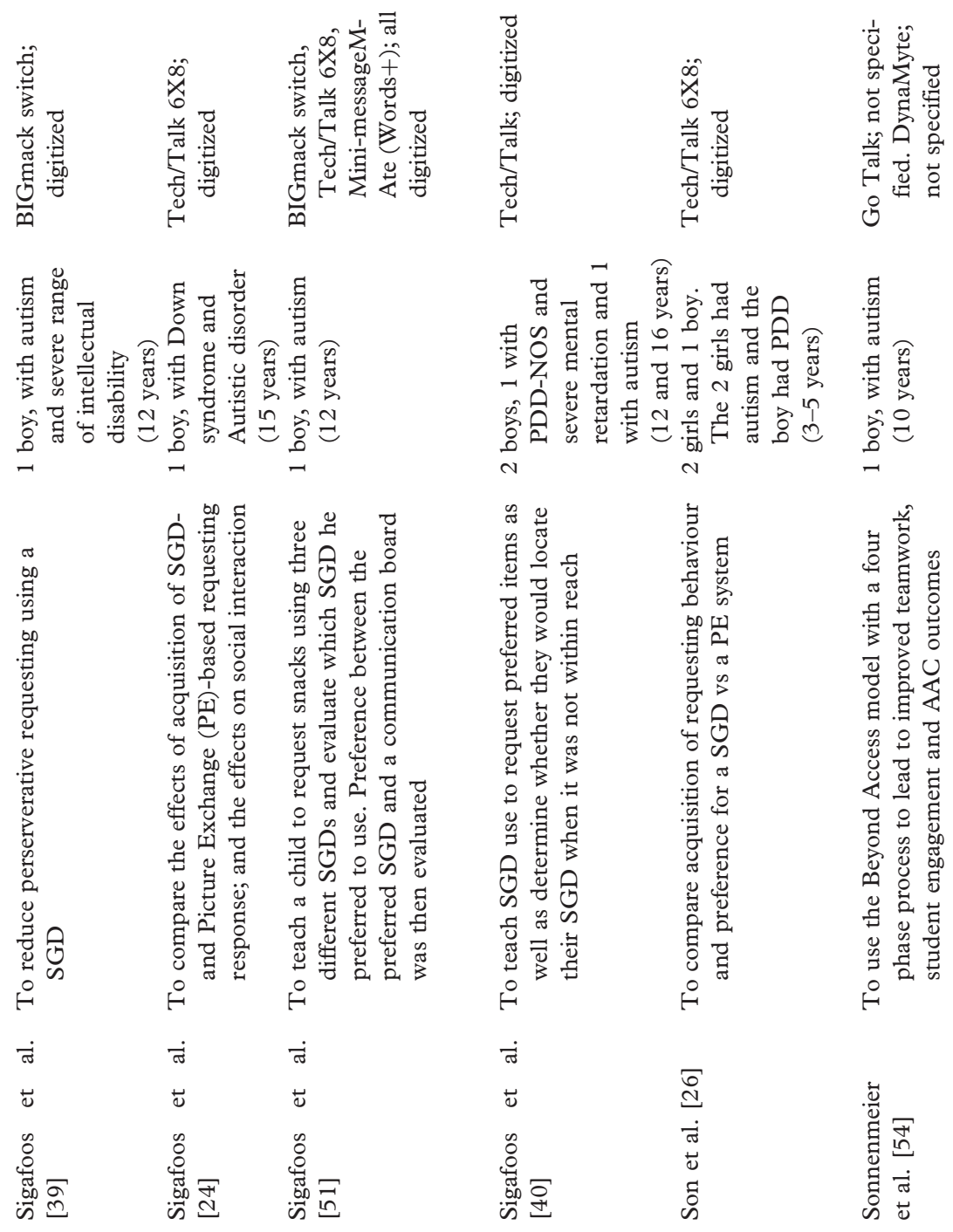
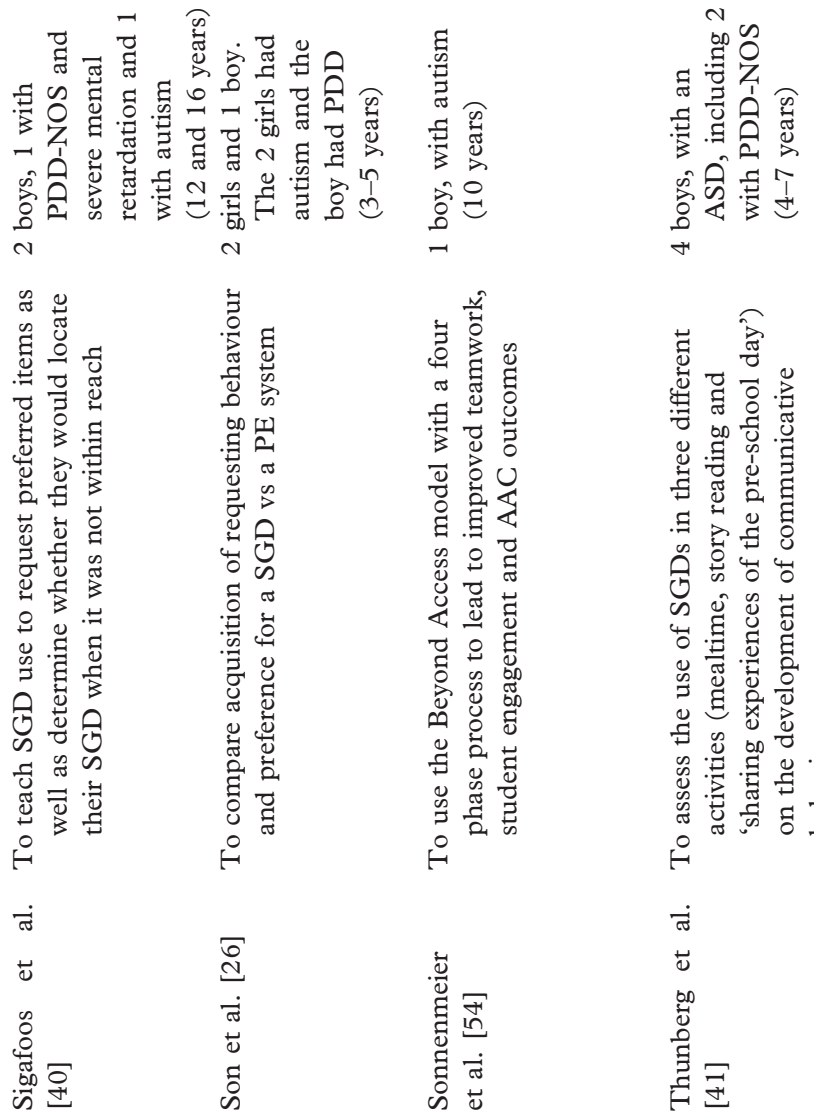

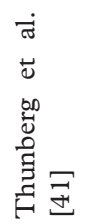
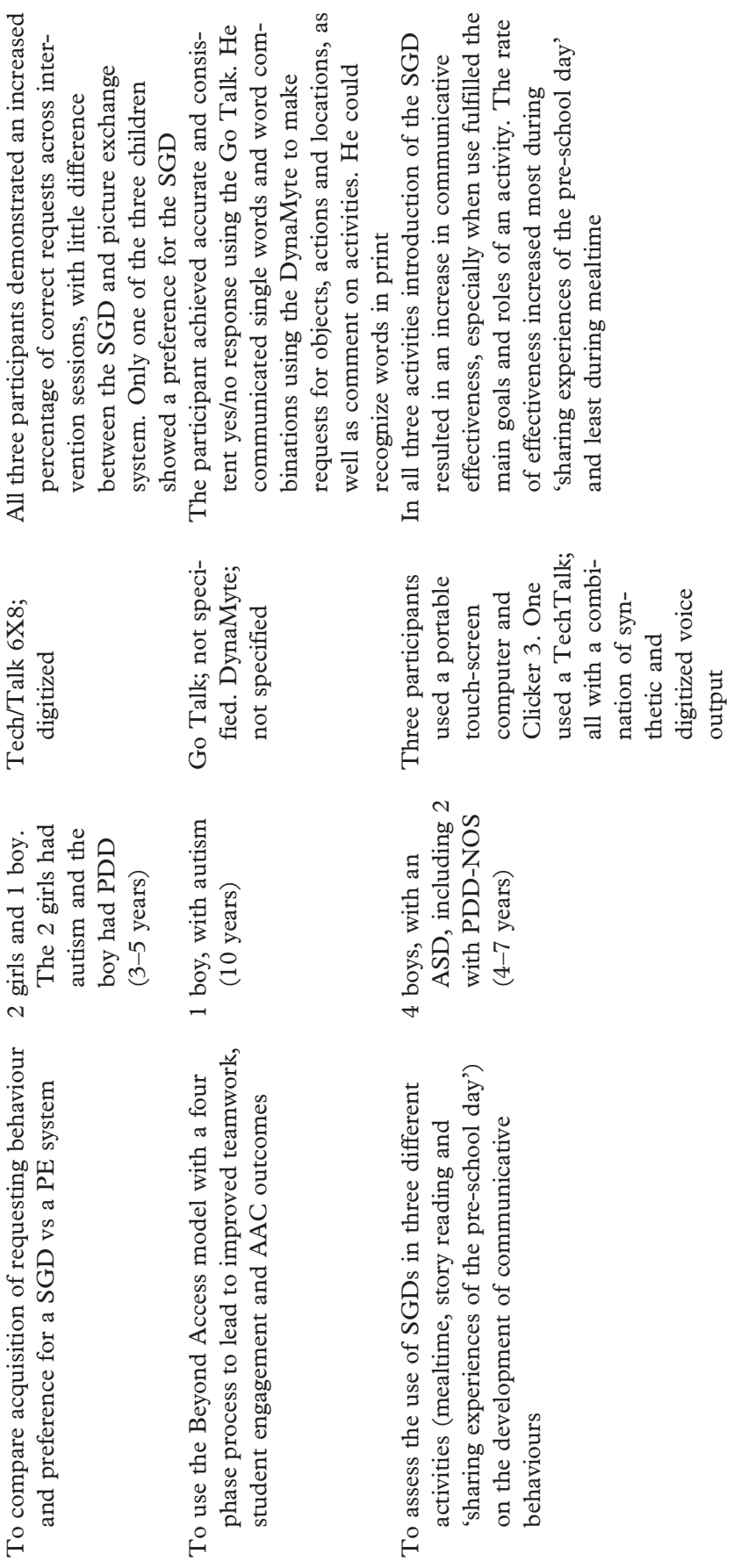


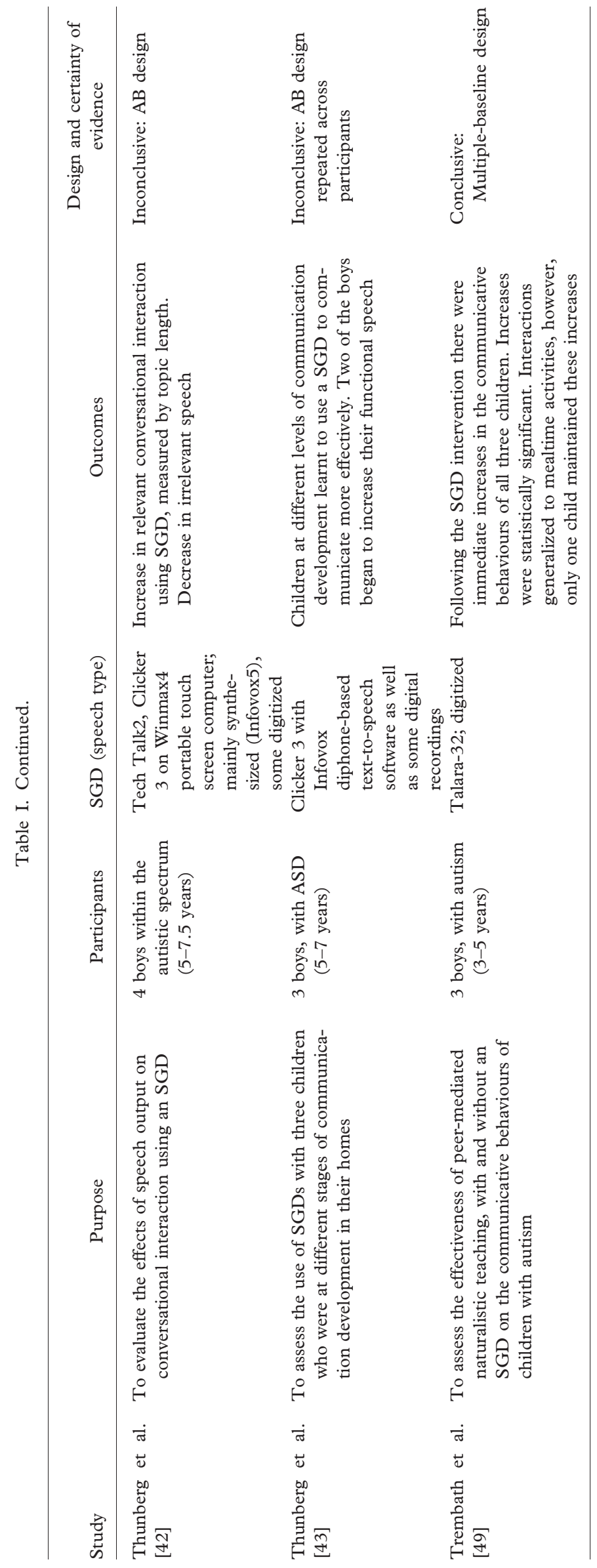


In this case age was estimated and calculated as 3 years. Two other studies $[22,36]$ only provided the age range of their participants and not individual ages. In these cases the average of the studies' sample was used to calculate the overall average. Sample sizes from the 23 articles ranged from one to five participants. Just under half $(n=9,39.1 \%)$ had one participant. Only one study [38] had five participants.

\section{Settings}

The most common setting for intervention was in participants' pre-schools or schools $(n=18,69.2 \%)$. For the three studies $[10,44,45]$ that were conducted in multiple settings the settings were counted separately (there were therefore 26 settings for the 23 studies). Six (23.1\%) studies [22, 41-44, 46] were undertaken in the participant's homes, one [10] intervention was undertaken in the community and one in a hospital [45].

\section{Mode of communication}

A total of 14 different types of SGDs were used in the 29 individual interventions reported. Several used more than one type of SGD per study and more than one speech type (i.e. digitized and synthetic). In such cases each SGD and speech type was recorded separately. Of the various SGDs used, the Tech/Talk $6 \mathrm{X} 8$ had the highest frequency $(n=6$, $20.7 \%$ ), followed by the BigMack $(n=4,13.8 \%)$ and both the GoTalk and touch screen computer with Clicker 3 each being used three times (10.3\%). The CheapTalk 4 Inline Direct and LightWRITER SL35 were each used twice $(6.9 \%)$. Finally the SpeakEasy, Introtalker, Four Button Touch Talk Direct, Black Hawk, The Vantage, MinimessageMATE (Words+), DynaMyte and Talara-32 were all used once (3.4\%). One study [5] did not specify what sort of SGD was used. Sixty-two per cent $(n=18)$ of studies used digitized voice-output, while $20.7 \%(n=6)$ of studies used synthesized voice-output and $17.3 \%(n=5)$ did not specify voice-output.

Sixty-one per cent $(n=14)$ of the studies provided some rationale and justification for the type of SGD selected. Of these, six studies [10, 25, 36, 45, 47, 48] based SGD selection on the participant's motor skills and resulting ability to depress the SGD keys. Five studies [25, 41-43, 49] based SGD selection on the participant's current vocabulary and level of communication skills, while four studies [37, 38, $50,51]$ chose SGDs with characteristics that related to the aims of the study. For example, Schlosser et al. [38] chose the Vantage because the device could be held constant within and across all planned replication studies while only manipulating the type of voice output (synthetic, digitized or no-speech).

\section{Communication skills taught to the participants}

Targeted communication skills were classified into broad categories, including: (a) requesting preferred items (e.g. food and snacks), activities, actions and locations, (b) conversation and social commenting involving single words, short phrases or sentence starters, (c) answering questions (e.g. yes/no questions), (d) spelling, (e) reducing perserverative requesting and irrelevant speech, (f) increasing natural speech and (g) other communicative behaviours (e.g. gestures, engagement in conversation, turn-taking, communicative form, function and effectiveness). Studies were also classified in terms of certain skills surrounding SGD use, including (a) independent SGD use (initiations and responses), (b) SGD use as a communication repair strategy, (c) location of the SGD and (d) SGD preference. Many of the studies taught more than one of the skills listed above. In such cases the skills were counted separately. Over half $(n=16$, $69.5 \%$ ) of the articles taught participants some form of requesting as the primary communication skill. This was followed by conversation and social commenting $(n=6,26 \%)$.

\section{Intervention procedures}

Sixty per cent $(n=15)$ of the articles used some form of the following operant/behavioural instructional procedures to teach the use of an SGD: (a) presenting an opportunity or discriminative stimulus, (b) prompting a communicative behaviour, (c) fading prompts and (d) providing reinforcement for correct communicative behaviour. Within this broad class of behavioural procedures some studies also identified more specific techniques as their core teaching mechanism, including (a) functional behaviour analysis (FBA) and functional communication training (FCT) [10, 46, 47], (b) graduated guidance procedure $[24,45,48]$, (c) least to most prompting [22, 40, 51], (d) most to least prompting [44], (e) error correction procedure [52], (f) constant time delay method [38], (g) response interruption technique to reduce requesting [39], (h) spelling taught using a SGD and implementing a 'copy-covercompare' method [37, 50], (i) modelling by an adult [41-43] and (j) modelling by a peer [49].

Several studies focused on staff training, employing several techniques to teach SGD use. These studies moved away from typical behavioural techniques, instead focusing on (a) time-delay milieu teaching or enhanced milieu teaching [36, 53], (b) incidental or naturalistic teaching [25] and (c) The Beyond Access model [54]. 


\section{Outcomes}

Outcomes were classified and ranked into one of three outcome categories: (a) positive outcomes in which target communication skill(s) improved for all participants, (b) negative outcomes in which none of the participants improved in the target communication skill(s) and, finally, (c) mixed outcomes in which improvement was evident for some, but not all participants in the study or in which some target skills improved and others did not. Eighty-seven per cent of studies $(n=20)$ reported positive outcomes and $13 \%(n=3)$ reported mixed outcomes. Within these mixed outcomes only one of the participants did not learn to use the SGD [44]. None of the reviewed studies reported negative outcomes.

\section{Follow-up and generalization}

Seven $(30.4 \%)$ of the studies [25, 36-38, 45, 47, 50] undertook some form of follow-up. Not all studies mentioned when follow-ups occurred. However, for those that did, follow-up occurred from 1 week after intervention $[38,50]$ to 3 months after intervention [47] and continued for 3 weeks [38, 50] through 1 year [25] post-intervention. All maintenance data was successful to some extent, although some results varied between participants.

Ten studies (43.5\%) [10, 36, 40, 44, 46-50, 52] undertook some form of generalization. Several of the studies reporting generalization were anecdotal in nature where some form of natural generalization was found without any specific testing [10, 40, 46, $48,52]$. Generalization across settings occurred for five studies [10, 36, 44, 47, 49], across people for one study [40] and across tasks for five studies [36, 46, 48, 50, 52]. All generalization data were successful to some extent, although some results were variable between participants and not all studies collected generalization data for all participants $[44,52]$.

\section{Reliability and treatment integrity}

Ninety-six per cent $(n=22)$ of studies reported reliability of data collection with respect to the dependent variables, such as collecting interobserver agreement (IOA). Eighty-six per cent $(n=19)$ of these studies reported average rates of inter-observer agreement above the generally accepted standard of $80 \%$ reliability [55]. Only one study [36] did not collect any reliability data. Fewer studies (39\%, $n=9$ ) reported treatment integrity data for the accurate implementation of intervention procedures [24, 37-39, 43, 46, 50, 52, 53]. All of these studies reported high treatment integrity scores of over $80 \%$ correct implementation.
Design and certainty of evidence

Twenty ( $87 \%$ ) of the 23 studies used some variation of a single-case design [55] to evaluate the effects of the SGD intervention on communication. Certainty of evidence was based on whether or not the study included a recognized experimental design. Studies were classified as conclusive if they systematically introduced and removed the intervention (e.g. $\mathrm{ABAB}$ ) [39] or the independent variable was sequentially introduced in accordance with a concurrent baseline (e.g. multiple-baseline, multipleprobe or alternating treatments design) [10, 22, 24, 25, 36-38, 40, 44-51, 53]. Studies were classified as inconclusive if they used intervention only, A-B or case study (narrative) designs [41-43, 52, 54]. According to these criteria, $18(78 \%)$ of the 23 studies were deemed conclusive, while five (22\%) of the 23 studies were deemed inconclusive, as they either involved a pre-experimental A-B design [42, $43]$ or employed a case study design $[41,52,54]$.

\section{Discussion}

The purpose of the current review was to provide an overview of the research surrounding communication interventions involving the use of SGDs for children with ASD. The systematic search identified 29 interventions reported in 23 studies, with a total of 51 participants published between 1998-2009. It therefore appears that in recent years there has been a rapid increase in research related to SGDs as an AAC intervention for children with ASD. It is interesting to note that such extensive growth has occurred after several articles in a 2001 issue of Focus on Autism and Other Developmental Disabilities highlighted the apparent need for more empirically-based applied research specific to AAC and ASD [6, 20, $27,56,57]$. In terms of the quality of evidence, the majority of studies reviewed here implemented experimental designs and achieved clear intervention results, where positive outcomes and certainty of evidence were both high $(87 \%$ and $78 \%$, respectively). As a result, $78 \%$ of studies were classified as demonstrating conclusive evidence. However, because not all studies demonstrated positive outcomes as well as conclusive evidence in relation to experimental design, these encouraging findings should still be interpreted with caution.

This review identified several trends, including (a) a clear tendency for targeting requesting as the main communication skill taught, (b) that instructional approach reflects the communication skill being taught, (c) the need to incorporate generalization and maintenance strategies into treatment procedures, (d) the development of preference studies to enable self-determination in AAC 
interventions and (e) the predominance of single-case designs and resulting individual outcomes. There appear to have been no large-scale randomized control trials evaluating SGDs for children with ASD. These trends have implications for practice and future intervention research.

In relation to the first trend, the prevalence of research studies targeting requesting skills reflects findings of other reviews where the greatest group of intervention studies focused on teaching simple requests using various modes of AAC to individuals with ASD [7, 18, 23]. This is not surprising considering it has been recommended that interventions begin by teaching a simple requesting response if it does not develop naturally [57, 58]. However, requesting is predominantly the beginning and end of AAC interventions for individuals with ASD; it is often the only communicative skill taught $[59,60]$. Future research should examine the transition from teaching early requesting skills to more advanced communication using SGDs. In order to do so expectations of the child's propensity to learn more advanced language skills may need to be raised [60]. Overall this review suggests that to maximize effectiveness of SGD selection and intervention, clinicians should consider a multitude of factors including considering children's preferences for different SGDs, decreasing learning demands, representation of language concepts, organization, navigation, selection technique and output [26].

Over half the articles analysed within this review provided some rationale and justification for the type of SGD selected. SGD selection was often based on participants' motor skills and their resulting ability to use the SGD, as well as their level of communication skills in relation to complexity of the SGD selected. This selection process has implications for practitioners who are faced with selecting a specific SGD for students with ASD. By considering the individual's motor skills the practitioner can ensure the individual can easily activate and navigate the SGD. The consideration of an individual's current communication skills and future communication goals is critical in making it possible to move beyond targeting requesting skills to teach more complex communication. In this way one can ensure every individual is provided with a 'viable, robust, flexible, and generative communication system that will support long-term language development' ([60], p. 225).

In terms of the second trend, intervention procedures were classified into two main categories: behavioural approaches, such as discrete-trial training, or naturalistic approaches, such as milieu teaching. Distinct patterns emerged from these studies with respect to the instructional procedures used and the communication skills taught. Studies that used discrete-trial training most commonly taught requesting of preferred items, whereas studies that utilized a naturalistic instructional approach (e.g. peer and adult modelling) more frequently taught simple social initiations and conversational skills. This reflects a similar trend for children with other types of developmental disabilities [61]. It could be that operant instructional procedures are best suited for teaching initial requesting behaviours and when communication becomes more complex in nature a naturalistic approach is more effective. Future research is recommended to determine which instructional strategy is best suited for the level of communication skills being taught.

The third trend illustrates that, while a number of studies did collect some generalization $(43.5 \%)$ and maintenance $(30.4 \%)$ data, most studies were limited to teaching one communication skill within the school setting. Of the studies that did report generalization several were anecdotal in nature, where generalization was found without any formal testing, reflecting a 'train and hope' approach to generalization [29]. Future research should focus on programming SGDs with multiple messages that can be used across a range of settings (school, home and the community), as a purposeful strategy to assess if SGD use will generalize to other contexts. More follow-up data is also warranted to assess whether such skills will maintain over time.

The comparison of intervention approaches using SGDs vs other AAC strategies was analysed in several studies [22, 24, 44, 51] and presents another area with significant implications for practice and future research. These studies have demonstrated that children with ASD can show a preference for using different types of AAC communication. This is consistent with a fourth trend where enabling students to participate in the selection of their communication device is one means of promoting self-determination. Future research is recommended to determine whether individuals' preference for a specific device results in more effective and efficient device operation and development of communication skills.

Some of this preference research suggests that several individuals may prefer to use SGDs over other AAC modes [51], although other research has identified that this does not seem to be true for all learners [22]. Similarly, studies by Schlosser et al. [37] and Schlosser and Blischak [50] examining which feedback mode (auditory or visual) on an SGD had the most effective outcome on spelling support this notion, where results varied depending on the individual participant. This relates to the fifth trend that interventions can result in a range of outcomes and highlights the importance of individualized assessment. 
The current review demonstrates recent strength in empirically examining the effects of SGD interventions for students with ASD. The majority of studies reported improvements in the child's ability to use SGDs to communicate following intervention. An SGD has many advantages over other AAC modes of communication; in particular it is easily used by the student and understood by others making it a functional mode of communication [28]. These advantages together with the positive outcomes reported make SGD-based interventions a potentially effective option for teaching communication skills to children with ASD.

Declaration of interest: The authors report no conflicts of interests. The authors alone are responsible for the content and writing of this paper.

\section{References}

1. American Psychiatric Association. Diagnostic and statistical manual of mental disorders. Washington, DC: APA; 2000.

2. Peeters T, Gillberg C. Autism: Medical and educational aspects. London: Whurr; 1999.

3. National Research Council. Educating children with autism. Washington, DC: National Academy Press; 2001.

4. Weitz C, Dexter M, Moore J. AAC and children with developmental disabilities. In: Glennen S, DeCoste D, editors. Handbook of augmentative and alternative communication. San Diego, CA: Singular; 1997. pp 395-431.

5. Wing L, Attwood A. Syndromes of autism and atypical development. In: Cohen DJ, Donnellan AM, editors. Handbook of autism and pervasive developmental disorders. New York: Wiley; 1987. pp 3-19.

6. Sigafoos J, Drasgow E. Conditional use of aided and unaided AAC: A review and clinical case demonstration. Focus on Autism \& Other Developmental Disabilities 2001;16: $152-161$.

7. Mirenda P. Toward functional augmentative and alternative communication for students with autism: Manual signs, graphic symbols, and voice output communication aids. Language, Speech, \& Hearing Services in Schools 2003;34:203-216.

8. Rowland CM. Presymbolic communicators with autism spectrum disorders. In: Mirenda $\mathrm{P}$, Iacono $\mathrm{T}$, editors. Autism spectrum disorders and AAC. Baltimore: Paul H Brookes Publishing Co; 2009. pp 51-81.

9. Durand V. Functional communication training using assistive devices: Effects on challenging behavior and affect. Augmentative and Alternative Communication 1993;9: 168-176.

10. Durand V. Functional communication training using assistive devices: Recruiting natural communities of reinforcement. Journal of Applied Behavior Analysis 1999;32:247-267.

11. Romski M, Sevcik R, Smith A, Barker R, Folan S, BartonHusley A. The system for augmenting language: Implications for young children with autism spectrum disorders. In: Mirenda P, Iacono T, editors. Autism spectrum disorders and AAC. Baltimore: Paul H. Brookes Publishing; 2009. pp 219-246.

12. Beukelman D, Mirenda P. Augmentative and alternative communication: Supporting children and adults with complex communication needs. Baltimore: Paul H. Brookes Publishing Co.; 2005.

13. Llyod L, Fuller D, Arvidson H. Augmentative and alternative communication: A handbook of principles and practices. Boston: Allyn \& Bacon; 1997.

14. Schlosser R, Sigafoos J. Augmentative and alternative communication interventions for persons with developmental disabilities: Narrative review of comparative single-subject experimental studies. Research in Developmental Disabilities 2006;27:1-29.

15. Reichle J, Beukelman D, Light J. Exemplary practices for beginning communicators: Implications for AAC. Baltimore: Paul H. Brookes Publishing Co.; 2002.

16. Bondy A, Frost L. The picture exchange communication system. Focus on Autistic Behavior 1994;9:1-19.

17. Bondy A, Frost L. The picture exchange communication system. Behavior Modification 2001;25:725-744.

18. Lancioni G, O'Reilly M, Cuvo A, Singh N, Sigafoos J, Didden R. PECS and VOCAs to enable students with developmental disabilities to make requests: An overview of the literature. Research in Developmental Disabilities 2007;28:468-488.

19. Schlosser R, Sigafoos J, Koul R. Speech output and speechgenerating devices in autism spectrum disorders. In: Mirenda $\mathrm{P}$, Iacono T, editors. Autism spectrum disorders and AAC. Baltimore: Paul H. Brookes Publishing Co.; 2009. pp 141-170.

20. Ogletree B, Harn W. Augmentative and alternative communication for persons with autism: History, issues and unanswered questions. Focus on Autism \& Other Developmental Disabilities 2001;16:138-140.

21. Soto G, Belifore PJ, Schlosser R, Haynes C. Teaching specific requests: A comparative analysis of skill acquisition and preference using two augmentative and alternative communication aids. Education and Training in Mental Retardation 1993;28:169-178.

22. Son S, Sigafoos J, O'Reilly M, Lancioni G. Comparing two types of augmentative and alternative communication systems for children with autism. Pediatric Rehabilitation 2006;9:389-395.

23. Schlosser R. Roles of speech output in augmentative and alternative communication: Narrative review. Augmentative \& Alternative Communication 2003;19:5-27.

24. Sigafoos J, Green V, Payne D, Son S, O'Reilly M, Lancioni G. A comparison of picture exchange and speechgenerating devices: Acquisition, preference, and collateral effects on social interaction. Augmentative and Alternative Communication 2009;25:99-109.

25. Schepis M, Reid D, Behrmann M, Sutton K. Increasing communicative interactions of young children with autism using a voice output communication aid and naturalistic teaching. Journal of Applied Behavior Analysis 1998;31: 561-578.

26. Drager K, Light J, Finke E. Using AAC technologies to build social interaction with young children with autism spectrum disorders. In: Mirenda P, Iacono T, editors. Autism spectrum disorders and AAC. Baltimore: Paul H. Publishing Co; 2009. pp 247-278.

27. Schlosser R, Blischak D. Is there a role for speech output in interventions for persons with autism? Focus on Autism \& Other Developmental Disabilities 2001;16:170.

28. Rotholz D, Berkowitz S. Functionality of two modes of communication in the community by students with developmental disabilities: A comparison of signing and communication books. The Association for Persons with Severe Handicaps 1989;14:227-233.

29. Schlosser R, Lee D. Promoting generalization and maintenance in augmentative and alternative communication: A 
meta-analysis of 20 years of effectiveness research. Augmentative and Alternative Communication 2000;16: 208-226.

30. Schlosser R, Wendt O. Effects of augmentative and alternative communication intervention on speech production in children with autism: A systematic review. American Journal of Speech-Language Pathology 2008;17:212-230.

31. Schlosser R, Wendt O, Sigafoos J. Not all systematic reviews are created equal: Considerations for appraisal. EvidenceBased Communication Assessment \& Intervention 2007;1: 138-150.

32. Light J, Roberts B, Dimarco R, Greiner N. Augmentative and alternative communication to support receptive and expressive communication for people with autism. Journal of Communication Disorders 1998;31:153-180.

33. Millar D, Light J, Schlosser R. The impact of augmentative and alternative communication intervention on the speech production of individuals with developmental disabilities: A research review. Journal of Speech, Language \& Hearing Research 2006;49:248-264.

34. Schlosser R, Sigafoos J. Navigating evidenced-based information sources in augmentative and alternative communication. Augmentative and Alternative Communication 2009;25: 225-235.

35. Schlosser R, Sigafoos J. Evidence-based communication assessment and intervention-purpose and procedures. Evidence-Based Communication Assessment \& Intervention 2007;1:52-54.

36. McMillan J. Teachers make it happen: From professional development to integration of augmentative and alternative communication technologies in the classroom. Australasian Journal of Special Education 2008;32:199-211.

37. Schlosser R, Blischak D, Belfiore P, Bartley C, Barnett N. Effects of synthetic speech output and orthographic feedback on spelling in a student with autism: A preliminary study. Journal of Autism \& Developmental Disorders 1998;28: 309-319.

38. Schlosser R, Sigafoos J, Luiselli J, Angermeier $\mathrm{K}$, Harasymowyz U, Schooley K, Belfiore P. Effects of synthetic speech output on requesting and natural speech production in children with autism: A preliminary study. Research in Autism Spectrum Disorders 2007;1:139-163.

39. Sigafoos J, Ganz J, O’Reilly M, Lancioni G. Evidence-based practice in the classroom: Evaluating a procedure for reducing perseverative requesting in an adolescent with autism and severe intellectual disability. Australasian Journal of Special Education 2008;32:55-65.

40. Sigafoos J, O'Reilly M, Seely-York S, Edrisinha C. Teaching students with developmental disabilities to locate their AAC device. Research in Developmental Disabilities 2004;25: 371-383.

41. Thunberg G, Ahlsen E, Sandberg A. Children with autistic spectrum disorders and speech-generating devices: Communication in different activities at home. Clinical Linguistics \& Phonetics 2007;21:457-479.

42. Thunberg G, Ahlsén E, Sandberg A. Interaction and use of speech-generating devices in the homes of children with autism spectrum disorders - an analysis of conversational topics. Journal of Special Education Technology 2009;24:1-17.

43. Thunberg G, Sandberg A, Ahlsén E. Speech-generating devices used at home by children with autism spectrum disorders: A preliminary assessment. Focus on Autism \& Other Developmental Disabilities 2009;24:104-115.

44. Beck A, Stoner J, Bock S, Parton T. Comparison of PECS and the use of a VOCA: A replication. Education and Training in Developmental Disabilities 2008;43:198-216.

45. Sigafoos J, Didden R, O'Reilly M. Effects of speech output on maintenance of requesting and frequency of vocalizations in three children with developmental disabilities. Augmentative \& Alternative Communication 2003;19:37-47.

46. Olive M, Lang R, Davis T. An analysis of the effects of functional communication and a voice output communication aid for a child with autism spectrum disorder. Research in Autism Spectrum Disorders 2008;2:223-236.

47. Franco J, Lang R, O'Reilly M, Chan J, Sigafoos J, Rispoli M. Functional analysis and treatment of inappropriate vocalisations using a speech-generating device for a child with autism. Focus on Autism \& Other Developmental Disabilities 2009;24:146-155.

48. Sigafoos J, Drasgow E, Halle J, O’Reilly M, Seely-York S, Edrisinha C, Andrews A. Teaching VOCA use as a communicative repair strategy. Journal of Autism and Developmental Disorders 2004;34:411-422.

49. Trembath D, Balandin S, Togher L, Stancliffe R. Peermediated teaching and augmentative and alternative communication for preschool-aged children with autism. Journal of Intellectual and Developmental Disability 2009;34: 173-186.

50. Schlosser R, Blischak D. Effects of speech and print feedback on spelling by children with autism. Journal of Speech, Language, and Hearing Research 2004;47:848-862.

51. Sigafoos J, O'Reilly M, Ganz J, Lancioni G, Schlosser R. Supporting self-determination in AAC interventions by assessing preference for communication devices. Technology and Disability 2005;17:1-11.

52. Brady N. Improved comprehension of object names following voice output communication aid use: Two case studies. Augmentative \& Alternative Communication 2000;16: 197-204.

53. Olive M, de la Cruz B, Davis T, Chan J, Lang R, O'Reilly M, Dickson S. The effects of enhanced milieu teaching and a voice output communication aid on the requesting of three children with autism. Journal of Autism and Developmental Disorders 2007;37:1505-1513.

54. Sonnenmeier R, McSheehan M, Jorgensen C. A case study of team supports for a student with autism's communication and engagement within the general education curriculum: Preliminary report of the Beyond Access model. Augmentative \& Alternative Communication 2005;21: 101-115.

55. Kennedy C. Single-case designs for educational research. Boston: Pearson Education Inc; 2005.

56. Mirenda P. Autism, augmentative communication, and assistive technology: What do we really know? Focus on Autism \& Other Developmental Disabilities 2001;16: 141-151.

57. Koul R, Schlosser R, Sancibrian S. Effects of symbol, referent and instructional variables on the acquisition of aided and unaided symbols by individuals with autism spectrum disorders. Focus on Autism \& Other Developmental Disabilities 2001;16:162-169.

58. Sigafoos J, Mirenda P. Strengthening communicative behaviors for gaining access to desired items and activities. In: Reichle J, Beukelman D, Light J, editors. Exemplary practices for beginning communicators: Implications for AAC. Baltimore: Paul H. Brookes Publishing Co.; 2002. pp 123-256.

59. Sigafoos J, O’Reilly M, Drasgow E, Reichle J. Strategies to accept socially acceptable escape and avoidance. 
In: Reichle J, Beukelman D, Light J, editors. Exemplary practices for beginning communicators: Implications for AAC. Baltimore: Paul H. Brookes Publishing Co.; 2002. pp 157-186.

60. Mirenda P. A back door approach to autism and AAC. Augmentative \& Alternative Communication 2008;24:220-234.
61. Rispoli M, Franco J, van der Meer L, Lang R, Camargo S. The use of speech-generating devices in communication interventions for individuals with developmental disabilities: A review of the literature. Developmental Neurorehabilitation; 2010;13:276-293. 
Copyright of Developmental Neurorehabilitation is the property of Taylor \& Francis Ltd and its content may not be copied or emailed to multiple sites or posted to a listserv without the copyright holder's express written permission. However, users may print, download, or email articles for individual use. 


\section{APPENDIX C}

Rispoli, M., Franco, J., van der Meer, L., Lang, R., \& Carmargo, S. (2010). The use of speech generating devices in communication interventions for individuals with developmental disabilities: A review of the literature. Developmental Neurorehabilitation, 13, 276-293 


\title{
The use of speech generating devices in communication interventions for individuals with developmental disabilities: A review of the literature
}

\author{
MANDY JENKINS RISPOLI ${ }^{1}$, JESSICA H. FRANCO ${ }^{2}$, LARAH VAN DER MEER ${ }^{3}$, \\ RUSSELL LANG ${ }^{4}$, \& SÍGLIA PIMENTEL HÖHER CAMARGO ${ }^{1}$ \\ ${ }^{1}$ Texas A \& M University, Educational Psychology, College Station, TX, USA, ${ }^{2}$ Autism Community Network, \\ San Antonio, TX, USA, ${ }^{3}$ Victoria University of Wellington, New Zealand, Wellington, New Zealand, and \\ ${ }^{4}$ University of California at Santa Barbara, Santa Barbara, CA, USA
}

(Received 13 fanuary 2010; accepted 18 fanuary 2010)

\begin{abstract}
Objective: This review synthesizes communication interventions that involved the use of speech generating devices (SGD) for individuals with developmental disabilities.

Methods: Systematic searches of electronic databases, journals and reference lists identified 35 studies meeting the inclusion criteria. These studies were evaluated in terms of (a) participants, (b) SGD function, (c) SGD characteristics, (d) intervention procedures, (e) intervention results and (f) certainty of evidence.

Results: Across these studies, intervention was provided to a total of 86 participants aged 1-42 years. Communication skills targeted included requesting, social or conversational skills, labelling items and receptive language. Intervention approaches were categorized as using Discrete Trial Training, Milieu teaching or a combined instructional approach. Positive outcomes were reported in $86 \%$ of the studies with $54 \%$ of studies categorized as providing conclusive evidence.

Conclusion: This literature base is considered promising due to the large number of conclusive studies and the replication of intervention approaches.
\end{abstract}

Keywords: developmental disability, speech generating device, voice output communication aid, communication

\section{Resumen}

Objetivo: Esta revisión sintetiza las intervenciones de comunicación involucradas en el uso de los dispositivos de generación del habla (SGD) para individuos con discapacidades del desarrollo.

Métodos: Múltiples búsquedas sistematizadas en bases de datos electrónicas, revistas y listas de referencias identificaron 35 estudios que cumplían con los criterios de inclusión. Estos estudios se evaluaron en términos de (a) participantes, (b) función SGD, (c) características SGD, (d) procedimientos de intervención, (e) resultados de la intervención y (f) certeza de la evidencia.

Resultados: A través de estos estudios, se proporcionó intervención a un total de 86 participantes con edades comprendidas entre los 1 y 42 años de edad. Las habilidades de comunicación específicamente incluidas fueron habilidades de conversación, sociales y de petición, etiquetado de elementos, y lenguaje receptivo. Los enfoques de intervención se categorizaron mediante el uso de entrenamiento de ensayo discreto, de enseñanza dentro de ambiente natural o un enfoque combinado de enseñanza. Se reportaron resultados positivos en $86 \%$ de los estudios y un $54 \%$ de los estudios se incluyeron en la categoría de aportar evidencia concluyente.

Conclusión: Esta base de literatura es considerada prometedora debido al gran número de estudios concluyentes y a la replicación de los enfoques de intervención.

Palabras clave: discapacidad del desarrollo, dispositivo de generación del habla, dispositivo de ayuda de comunicación de producción de la voz, comunicación 


\section{Introduction}

Individuals with developmental disabilities often have severe communication impairments and may fail to develop speech $[1,2]$. In addition to communication delays, individuals with developmental disabilities often experience deficits in other areas, which may further preclude communication (e.g. cognition, oral motor skills, maintaining eye contact, initiating joint attention) [3, 4]. Communication impairment has been linked to an increased risk of challenging behaviour and reduced opportunities for community involvement $[5,6]$.

Given that communication deficits are prevalent and often persistent, individuals with developmental disabilities are frequently candidates for augmentative and alternative communication (AAC). AAC refers to communicative systems or strategies which may be used to supplement an individual's existing speech or as a primary communication alternative to speech [7-9]. AAC can include unaided communication (e.g. hand or body signals) or aided communication. Aided communication involves the use of external equipment with a communicative function, such as the exchange of pictures [10] or activation of a microswitch linked to a device that generates speech $[8,11]$.

A speech generating device (SGD), also referred to as voice output communication aid (VOCA), is a portable electronic device that, when activated by the individual intending to communicate, will produce a previously recorded or digitized spoken message. These generated messages may serve a number of communicative functions, such as requesting, commenting, greeting or answering questions [12]. A large variety of SGDs capable of serving one or multiple communicative functions exist. These devices vary in terms of the type of display (e.g. static vs dynamic), the number of communicative options presented, graphic symbols used, the use of digitized vs pre-recorded speech, as well as the shape and size of the device $[11,13,14]$.

A great deal of intervention research investigating the use of SGDs with individuals with developmental disabilities exists and at least five excellent reviews focusing on various issues related to the use of AAC devices have been written [11, 14-17]. Schlosser and Lee [15] examined the efficacy of 50 AAC studies published between 1976-1995. Their review offers support for the use of AAC in general, but does not focus on individual modalities of AAC (e.g. SGDs). Lancioni et al. [11] focused solely on the use of AAC devices and the picture exchange communication system (PECS) to teach requesting behaviours to individuals with developmental disabilities. They found that while the majority of studies produced positive outcomes, many had methodological flaws suggesting the results should be interpreted with caution. Lancioni et al. [16] reviewed 10 SGD studies and concluded that speech output systems have been successful and practical for users with developmental disabilities. A review of the most recent research that focuses specifically on the use of SGDs across a variety of communicative functions remains warranted. Additionally, no review to date has addressed which model of SGDs are being used and for what communicative functions. Access to such information would be important for practitioners as they consider the research base in determining which device is most appropriate for a specific individual.

To facilitate evidence-based practice in this important area, we herein provide a systematic review of studies in which individuals with developmental disabilities were taught to use a SGD. This focus on SGDs, as opposed to the broader focus on AAC, allows for an analysis of instructional strategies used to teach device operation. In addition to this focus and inclusion of the most recent research, this review will also add to the literature by examining the use of SGD devices across a variety of communicative functions. Each study included in the review is summarized in terms of (a) participants, (b) SGD function, (c) SGD characteristics, (d) intervention procedures, (e) intervention results and (f) certainty of evidence. A review of this nature has two main aims. First, it is intended to assist practitioners in their efforts to improve the communicative ability of individuals with developmental disabilities. Secondly, it aims to identify areas in need of future research efforts.

\section{Method}

\section{Search procedures}

Systematic searches were conducted in four electronic databases: Academic Search Complete, Education Resources Information Center (ERIC), Medline and PsychINFO. Publication year was not restricted, but the search was limited to Englishlanguage peer-reviewed studies. On all four databases, the terms 'augmentative communication', 'voice output', 'device', 'speech generating', 'functional communication training', 'SGD', 'AAC' or 'VOCA' were inserted into the Keywords fields.

Three additional search strategies were used in order to find other possibly relevant studies that may have been missed by the electronic search. First, ancestry searches through the reference lists of studies meeting inclusion criteria (see Inclusion criteria below) were also conducted. Secondly, hand searches, covering the year 2009 were then completed for the journals that had published included studies in order to identify recent studies 
that were not yet available within the electronic databases. Finally, electronic database searches of authors of the studies meeting inclusion criteria were conducted to identify other potentially relevant work by these authors. This systematic search occurred during February and October 2009. This initial search yielded a sample of 284 studies. The abstracts of the 284 studies were reviewed to identify studies for inclusion.

\section{Inclusion criteria}

To be included in this review, the article had to describe a research study that included the provision of a communication intervention in which an SGD with at least one person with a diagnosis of a developmental disability other than autism spectrum disorder (ASD; for a review of communication interventions using SGDs with individuals with ASD see [18]). An SGD included any portable electronic device that produced recorded speech output when activated by a lever, switch or button. Communication intervention was defined as attempting to improve a person's communication skill. Communication skills were defined as (a) functional communicative acts, such as commenting, requesting, greeting and answering questions or (b) language skills such as syntax or morphology. Studies that targeted only literacy skills, such as rhyming, were excluded, because the focus of this review was communication and not literacy. Finally, studies that focused only on assessment or description of communication with a SGD were not included [19, 20]. In studies with multiple participants, only data pertaining to participants with developmental disabilities other than ASD were included. Of the original 284 studies identified during the systematic search, 35 met criteria for inclusion in this review.

\section{Data extraction}

Each included study was summarized in terms of the following features: (a) participant characteristics, (b) SGD function, (c) SGD characteristics, (d) intervention procedures, (e) intervention results and ( $f$ ) certainty of evidence. Various procedural aspects were also noted, including research design, inter-observer agreement, treatment fidelity, implementation setting, maintenance and generalization of effects. Main findings were summarized in terms of the extent to which communication skills were shown to improve. Certainty of evidence was evaluated by considering main findings in light of the research design and other methodological details. The ability of a study to provide certainty of evidence was rated as either 'inconclusive' or 'conclusive' [21-24]. This classification system was utilized in an effort to provide an overview of the quality of evidence across the corpus of reviewed studies [25].

Appraising the certainty of evidence followed a two-stage process. First, only studies that included an experimental design (e.g. multiple-baseline or ABAB) could be considered as having the potential to provide conclusive evidence. Thus, any study that lacked an experimental design was automatically classified as inconclusive. This included descriptive studies and studies using $\mathrm{A}-\mathrm{B}$ or intervention-only designs. Secondly, studies that utilized an experimental design also had to meet four additional standards to be classified as providing conclusive evidence. First, the data had to provide a convincing demonstration of an intervention effect. This determination was based on visual inspection of data trends within and across phases [26]. For example, there had to be a clinically significant improvement in communication when intervention was introduced. Secondly, if relevant, there had to be adequate inter-observer agreement data (i.e. agreement observations conducted across $20 \%$ of the sessions and agreement coefficients exceeding $80 \%$ ). Thirdly, the dependent and independent variables had to be operationally defined. Finally, intervention procedures had to be described in sufficient detail to enable replication.

\section{Inter-rater agreement}

The first and second authors made an initial determination as to whether each study identified in the search met the inclusion criteria. After this, the fourth author repeated the search and independently assessed each of the studies against the inclusion/ exclusion criteria. Agreement as to whether a study should be included or excluded was $100 \%$ (i.e. agreement was obtained on all 35 studies).

The first and second author extracted information to develop an initial summary of the 35 included studies. The accuracy of these summaries was independently checked by one of the remaining co-authors using a checklist that included the initial summary of the study and a number of questions regarding various details of the study (i.e. Is this an accurate description of the participants?, Is this an accurate description the targeted communication skills?, Is this an accurate description of the SGD?, Is this an accurate description of intervention procedures?, Is this an accurate description of the results of the intervention? and Is this an accurate description of the certainty of evidence?). Co-authors were asked to read the study and the summary and then complete the checklist. In cases where the summary was not considered accurate, co-authors 
were asked to edit the summary to improve its accuracy. The resulting summaries were then used to create Table I.

This approach was intended to ensure accuracy in the summary of studies and to provide a measure of inter-rater agreement on data extraction and analysis. There were 315 items on which there could be agreement or disagreement (i.e. 35 studies with nine questions per study). Agreement was obtained on 290 items (92\%). In the 25 instances where aspects of the summaries were considered inaccurate, changes were made to more accurately summarize the studies.

\section{Results}

Table I summarizes the participant characteristics, targeted communication skills, SGD characteristics, intervention procedures, results and certainty of evidence of these 35 studies.

\section{Participant characteristics}

Collectively, the 35 studies reviewed provided communication intervention to a total of 86 participants. The mean sample size was 2.4 participants per study (range 1-12). Participants' mean age was 12.9 years (range $=1.1-42$ years). The most common diagnosis was intellectual disability $(70 \%$ of the participants, $n=60)$. Within this category, $9 \%(n=8)$ of the participants had mild-to-moderate intellectual disability, $15 \% \quad(n=13)$ had moderate-to-severe intellectual disability, $42 \% \quad(n=36)$ had severeto-profound intellectual disability and for 4\% $(n=3)$ of participants the level of intellectual disability was not reported. Sixteen per cent of participants $(n=14)$ had a diagnosis of at least one of the following: cerebral palsy, encephalopathy or sensory impairment. Fourteen per cent of participants $(n=12)$ had a diagnosis of a developmental delay. Ten per cent of participants $(n=9)$ were diagnosed with a speech or language impairment or physical disability and $5 \%(n=4)$ with multiple disabilities or seizure disorder. Two per cent of participants $(n=2)$ had a diagnosis of Down syndrome. Finally, the diagnoses of Angelman syndrome, disruptive behaviour disorder, hydrocephalus, Pierre-Robin syndrome, spina bifida and submucal cleft palate were reported for one participant each. Fifty-five per cent of the participants $(n=47)$ had multiple diagnoses.

Participants were divided into one of four categories based on their communication skills prior to intervention: (a) non-verbal, (b) non-verbal with gestures, (c) limited verbal skills or (d) verbal. The non-verbal category included participants with a total absence of appropriate communication skills. Fifty-two per cent of participants $(n=45)$ were classified as non-verbal. Non-verbal with gestures included participants with no spoken language but who use gestures (such as pointing or manual signs) to communicate. Eighteen per cent of participants $(n=15)$ were classified as non-verbal with gestures. Limited verbal skills included participants with a spoken vocabulary of fewer than 10 words. Twentynine per cent of participants $(n=25)$ were categorized as having limited verbal skills. Participants who were categorized as verbal had more than 10 words in their vocabulary and used speech as a primary means of communication. One participant was classified as verbal.

\section{SGD function}

The communication skills targeted for intervention (i.e. dependent variables) were coded into one of five categories: (a) requesting attention, food, items, (b) social or conversational (e.g. increasing the number of conversational turns, staying on topic), (c) labelling items, (d) receptive (e.g. pointing to pictures, answering questions) or (e) multiple skill areas. The majority of participants $(58 \%)$ were taught requesting skills using their SGD $(n=50)$. Intervention targeted the production of social messages, such as 'thank-you' using the SGD for $27 \%$ of the participants $(n=23)$. Four per cent of participants were taught to label $(n=3), 7 \%$ were taught to use appropriate syntax $(n=6), 2 \%(n=2)$ were taught to answer questions during conversation and $2 \%$ $(n=2)$ were taught multiple skills.

\section{SGD characteristics}

Seventeen different types of SGDs were identified. These SGDs are described in Table I. However, discriminating among these devices was difficult, as the same SGD may be called different names by different companies. For example, 'Big Red' and 'Big Mac' are both single-switch SGDs that are likely identical in terms of the physical characteristics and display. Within the corpus of studies, the most commonly used $(46 \%)$ SGD was a single switch or microswitch $(n=39)$. Following this device, $14 \%$ of the participants used Portable Voice II with laptop $(n=12), 11 \%$ used Go Talk $(n=9), 5 \%$ used Touch Talker $(n=4), 4 \%$ used IntroTalker $(n=3)$ and $4 \%$ used Mega Wolf $(n=3)$. Each of the following devices was used by $2 \%$ of the participants $(n=2)$ : Alpha Talker, Super Wolf, Liberator, Macaw 3 and Wolf. One per cent of the participants $(n=1)$ used Speak Easy, Dynavox, Massage Mate, Keyboard 
M. F. Rispoli et al.

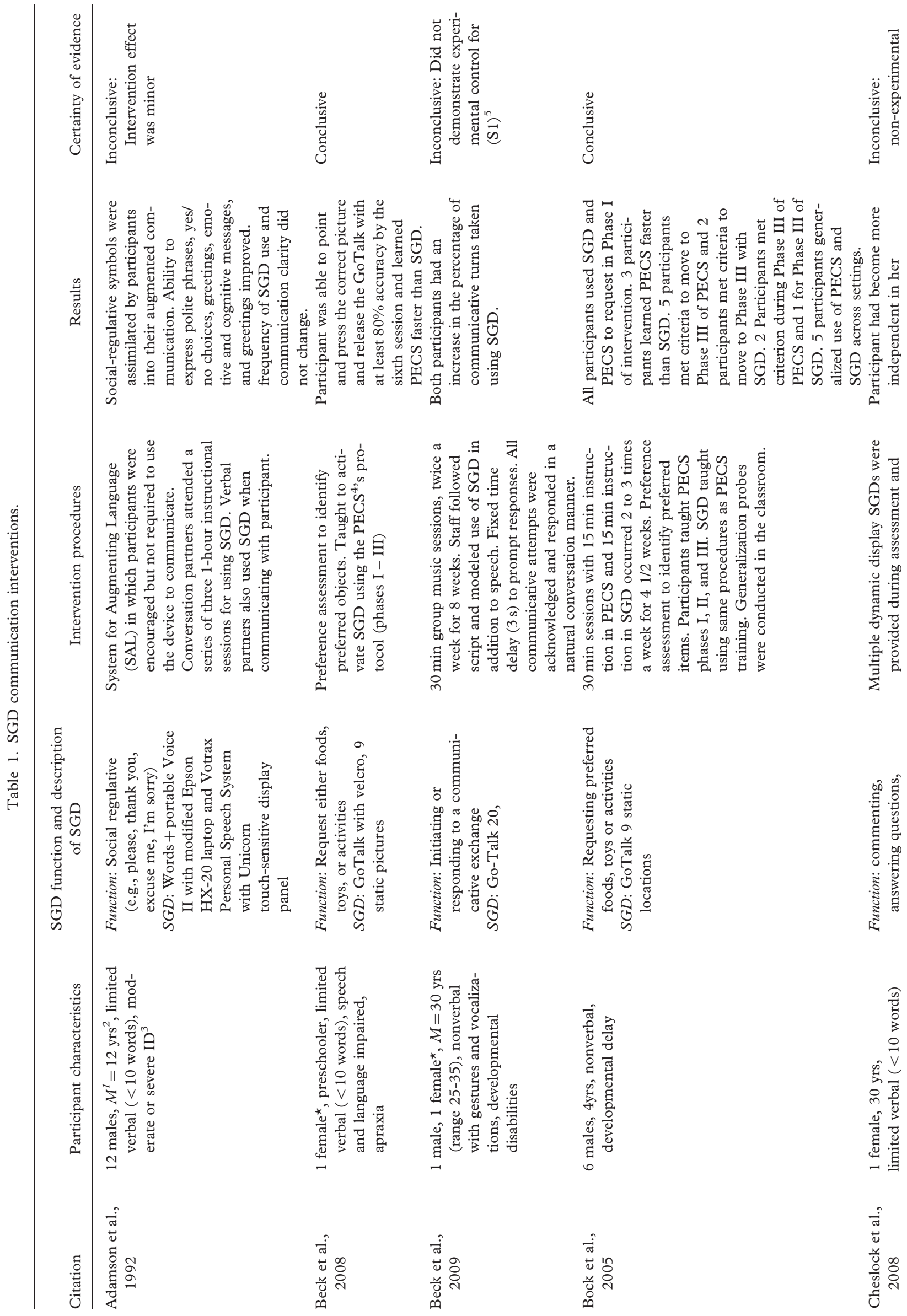




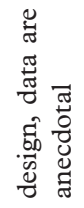
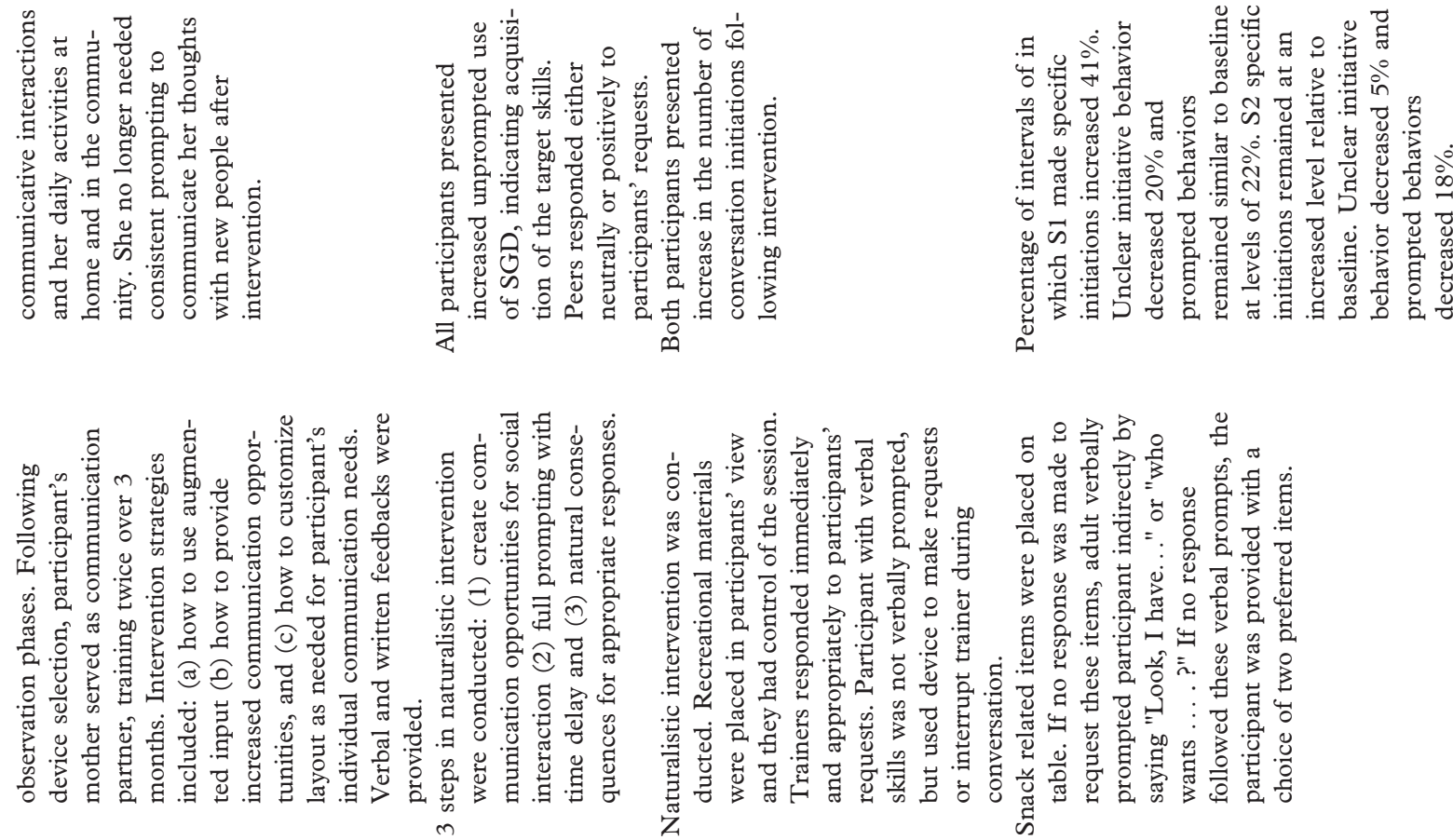

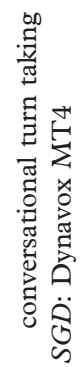
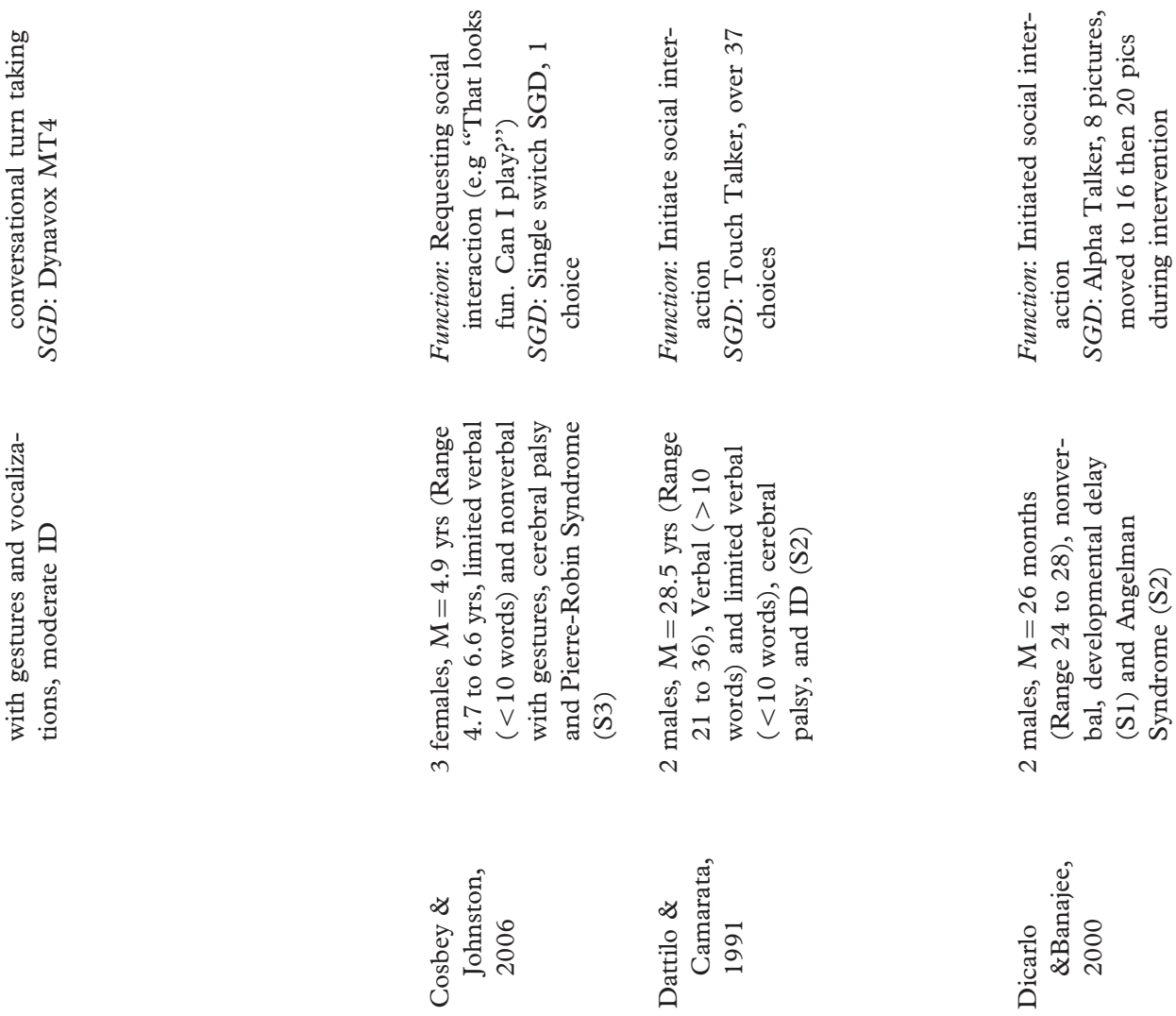

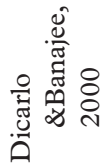




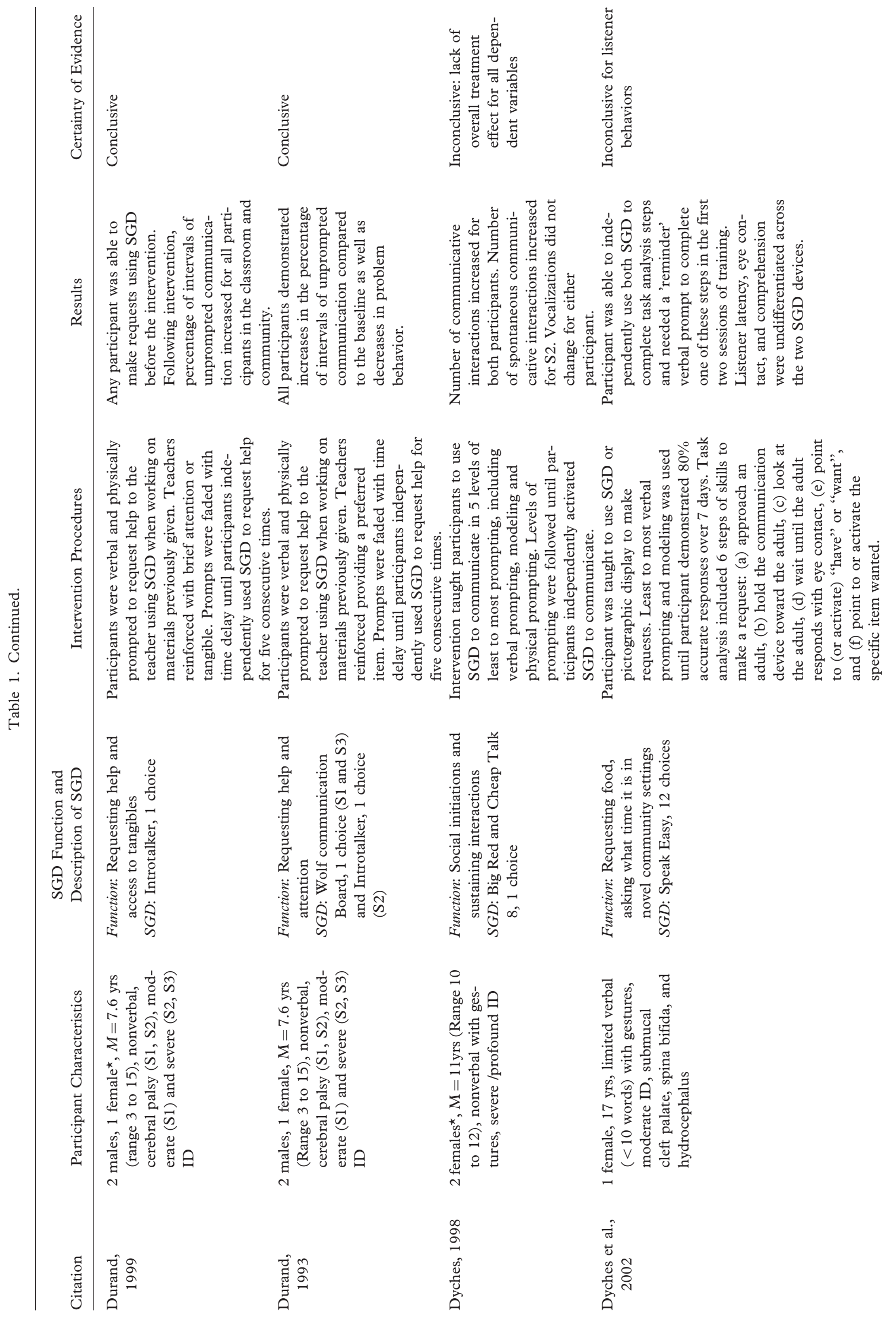



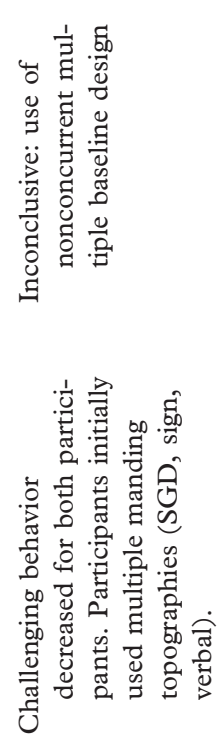
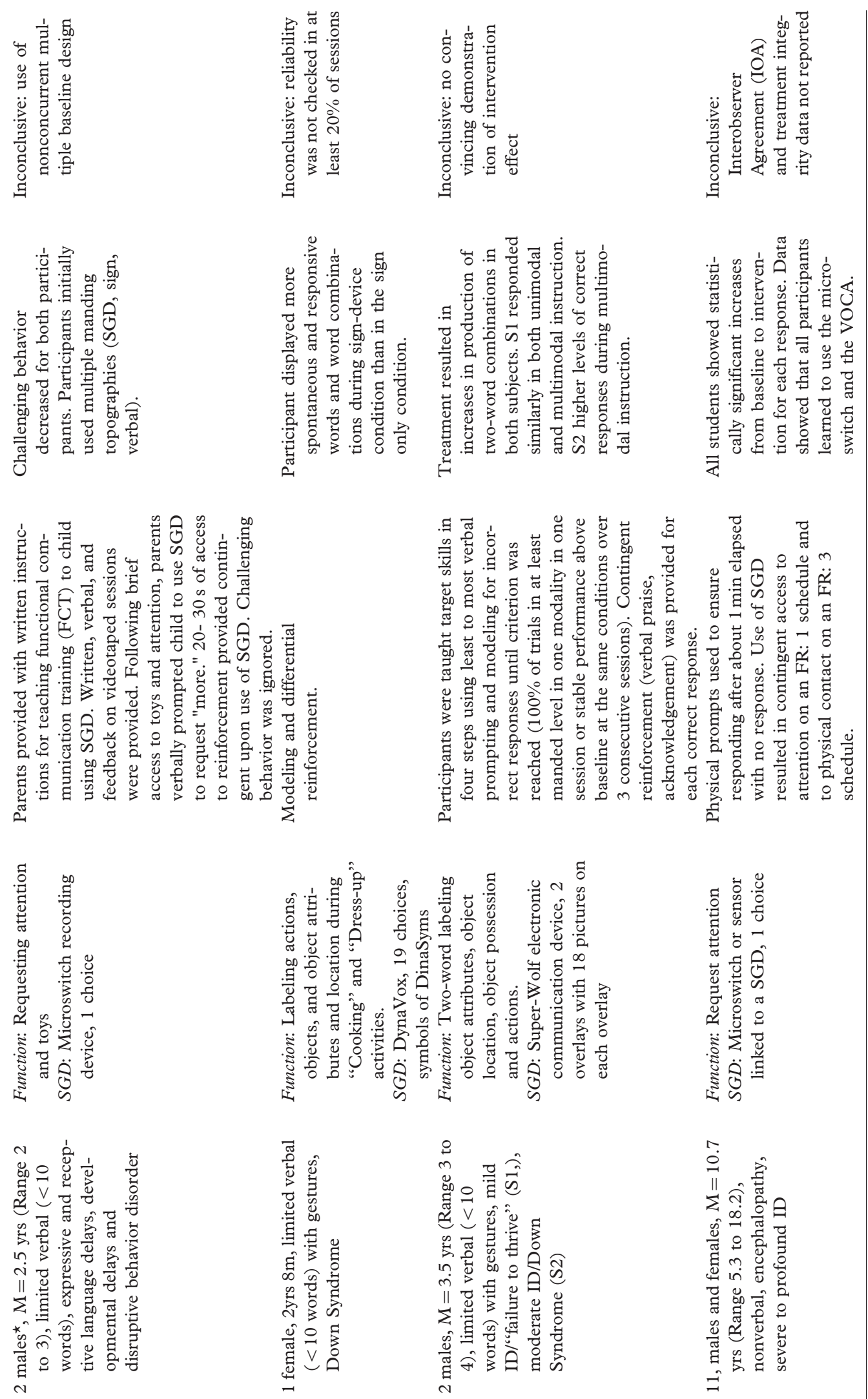

翌铰

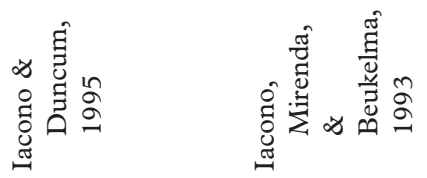


M. F. Rispoli et al.

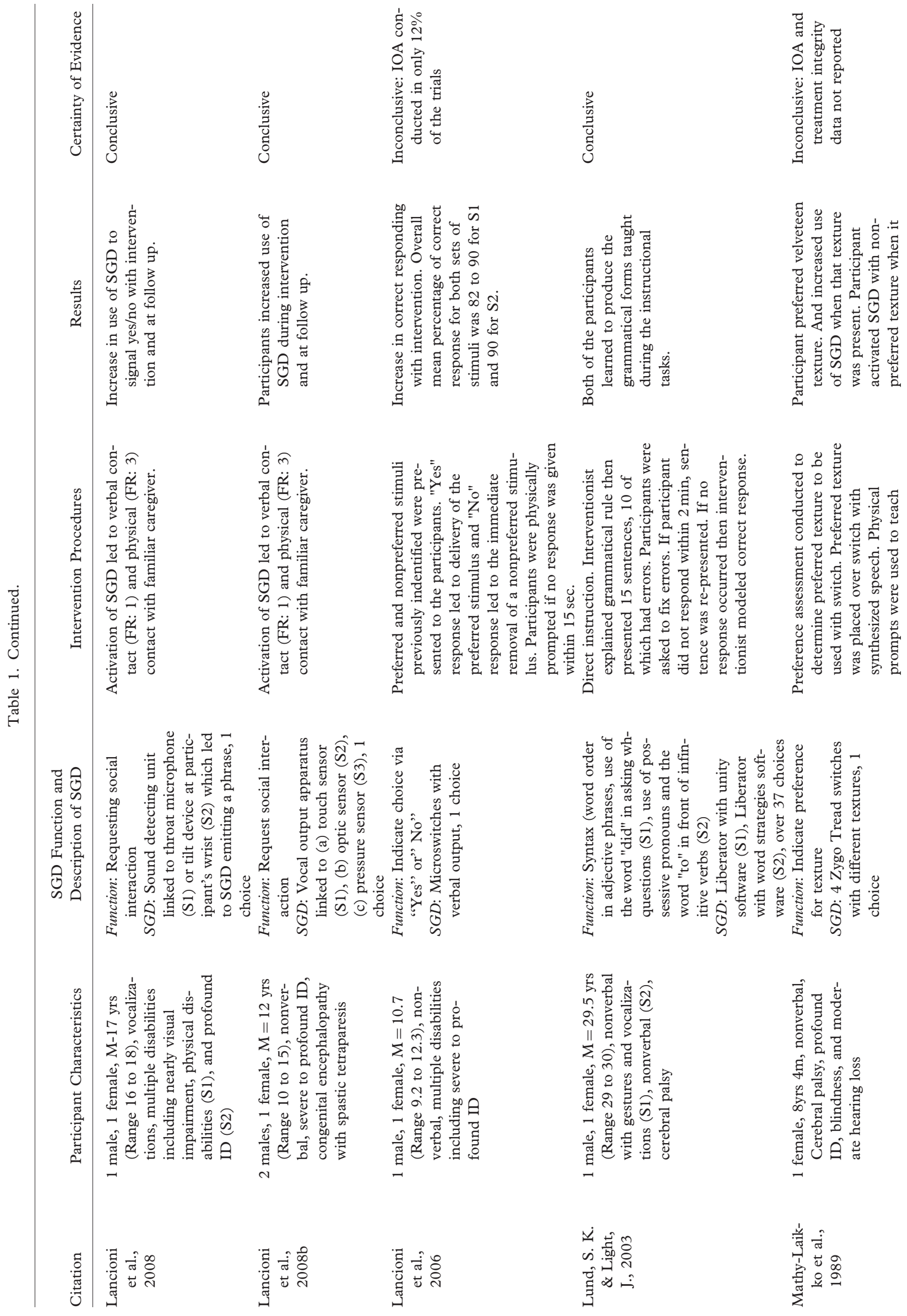



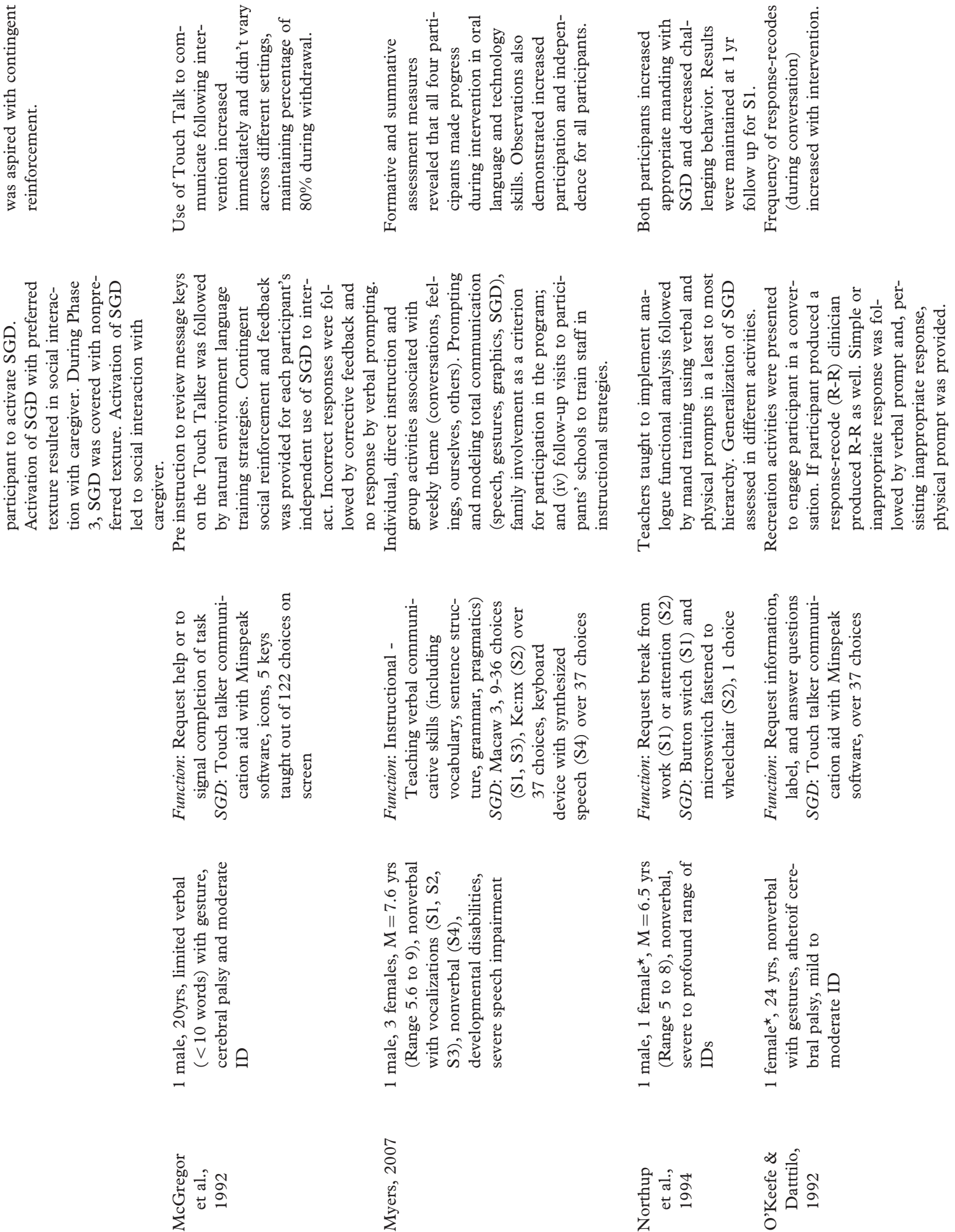

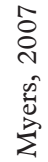

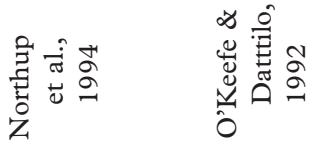




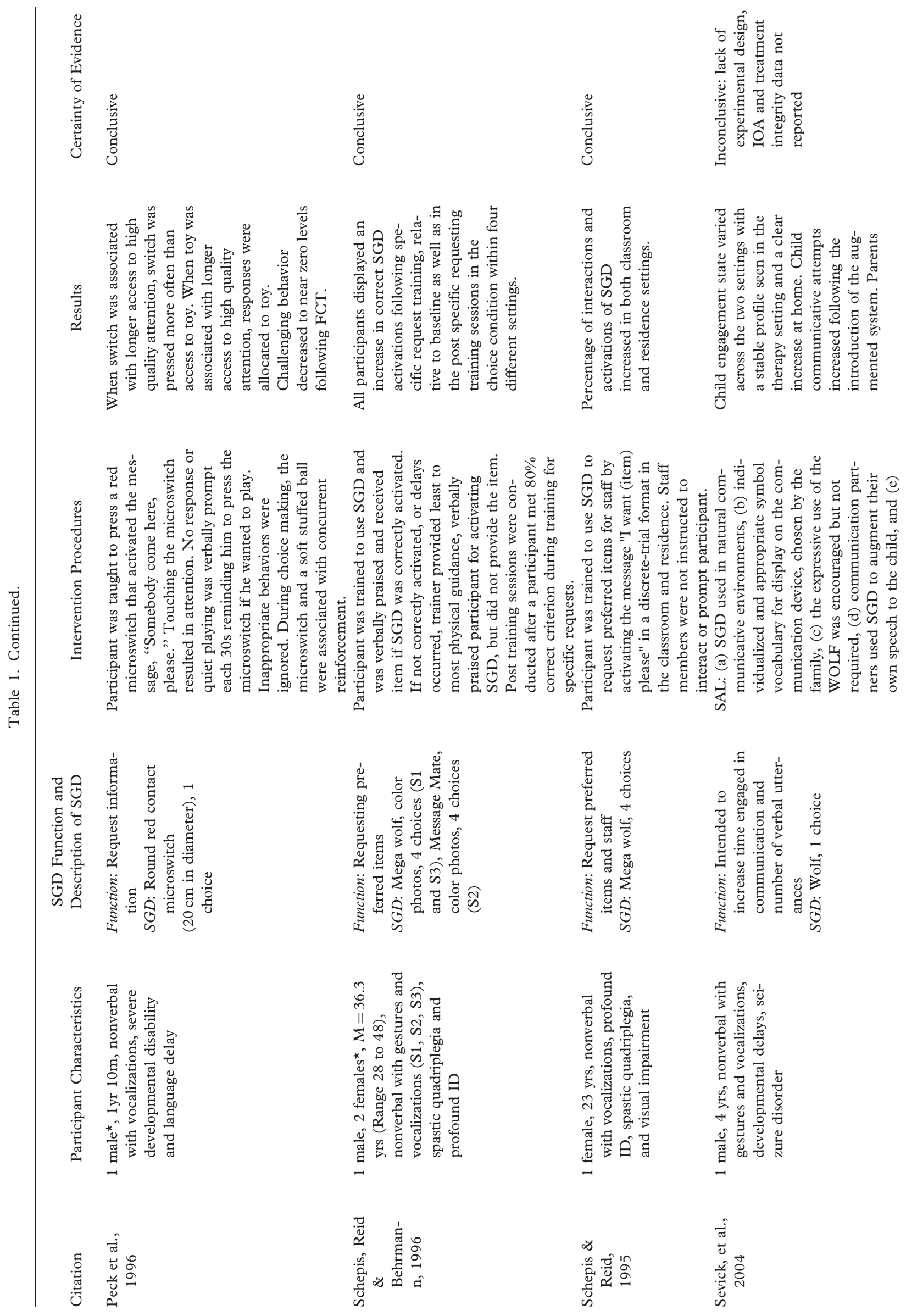




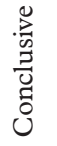

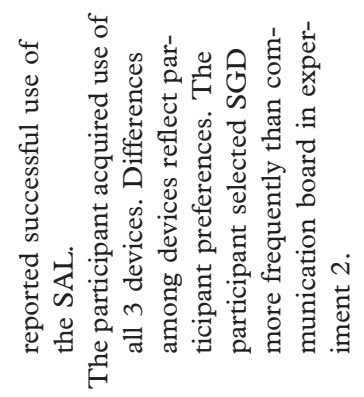

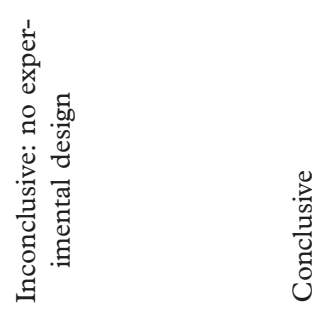

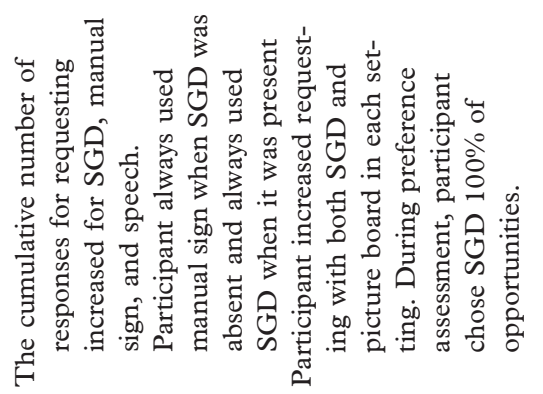

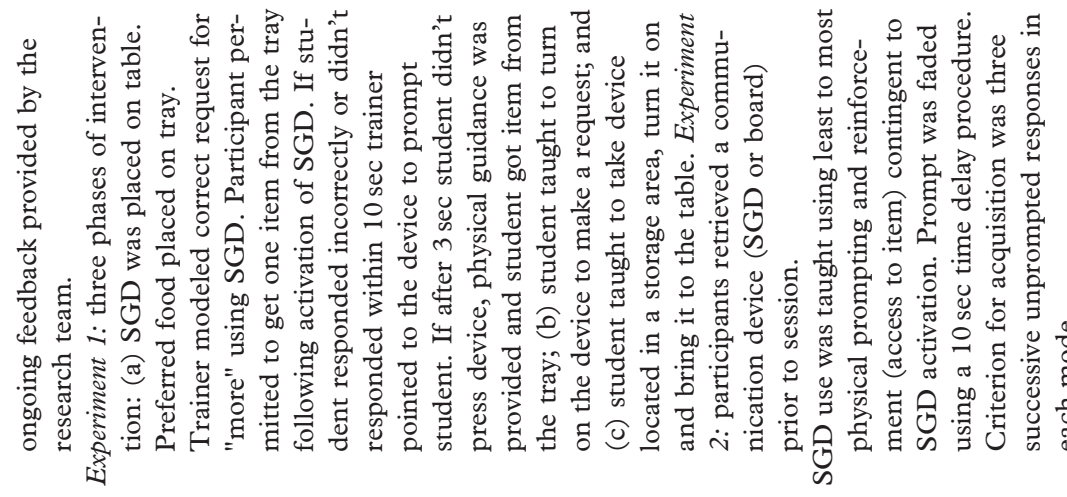
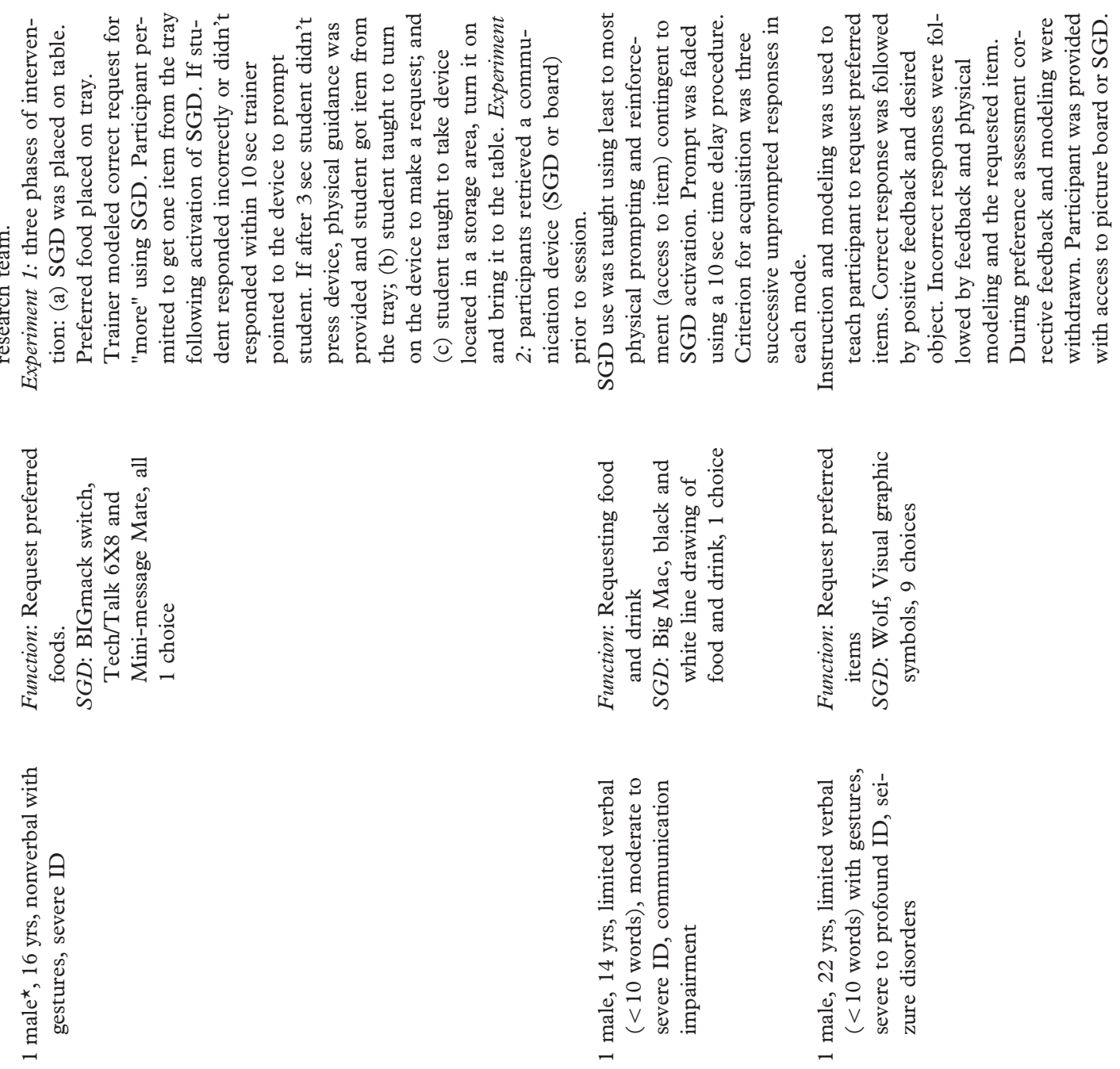

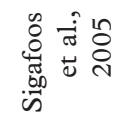

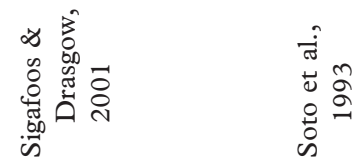




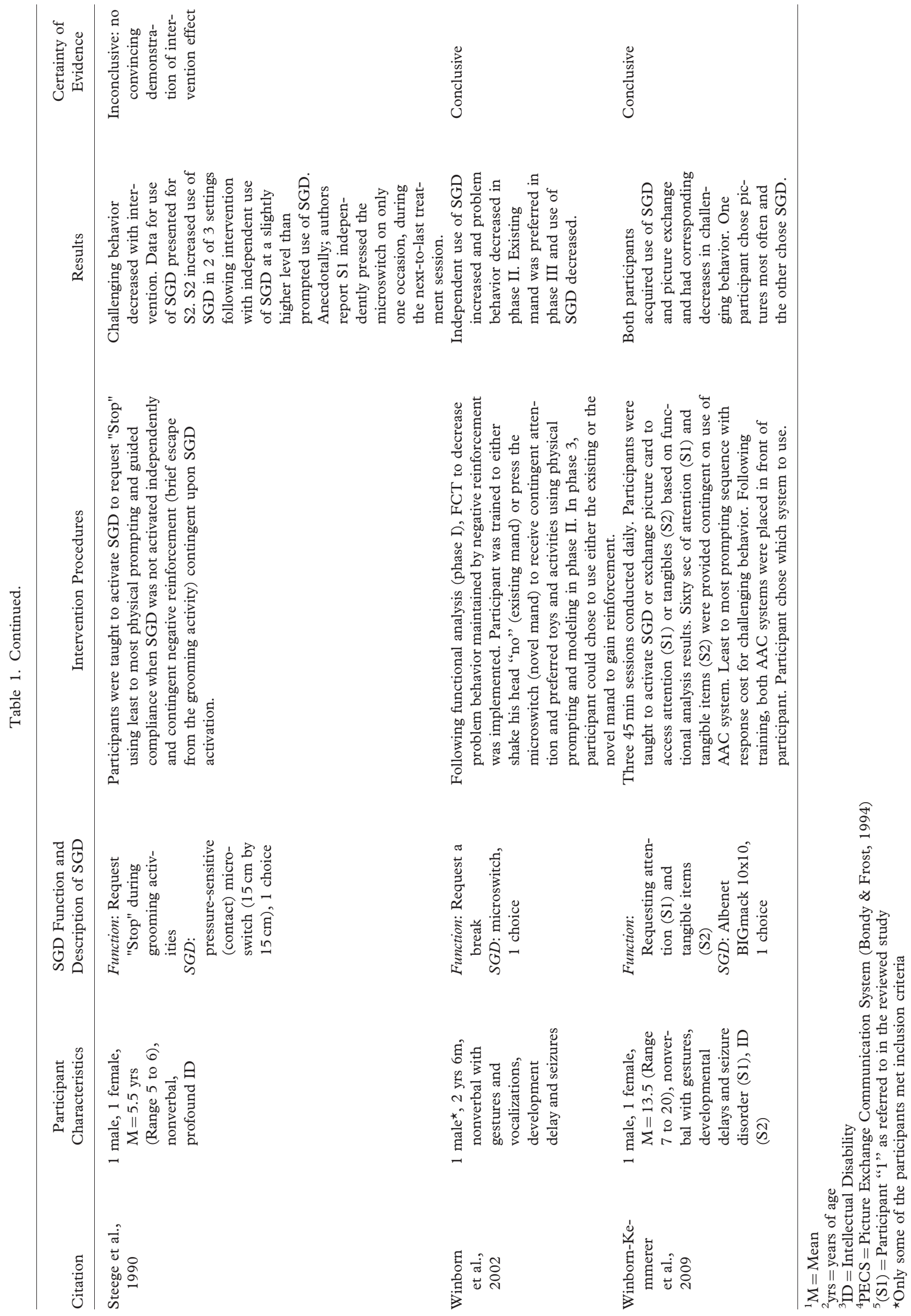


with synthesized speech, MT4 and Laptop with Ke:nx.

The number of choices or options available on the SGD also varied. Forty-six per cent of participants $(n=39)$ used SGDs with only one button or switch. Five per cent of participants $(n=4)$ had SGDs with two-to-eight choices, while $25 \%$ of participants $(n=22)$ used SGDs with nine-to-36 choices and $7 \%$ of the participants $(n=6)$ used an SGD with over 37 choices. Unfortunately, the exact number of choices could not be determined for $17 \%$ of the participants $(n=15)$.

Among participants who were non-verbal, $62 \%$ used single switch/microswitch $(n=28)$. The rest of the participants in this category used various devices, with $13 \%$ using the GoTalk $(n=6), 7 \%$ the IntroTalker $(n=3), 5 \%$ the Alpha Talker $(n=2)$, $5 \%$ the Macaw $3(n=2), 2 \%$ the Liberator $(n=1)$, $2 \%$ the Mega Wolf $(n=1), 2 \%$ a laptop with Ke: $n x$ $(n=1)$ and $2 \%$ a keyboard with synthesized speech $(n=1)$. Related to the number of choices, $62 \%$ of the participants with non-verbal communication skills used devices with a single choice $(n=28)$, $2 \%$ used devices with two-to-eight choices $(n=1)$ and $36 \%$ used devices with nine or more choices $(n=16)$.

Participants who were non-verbal but used gestures to communicate also utilized diverse types of devices. However, like the other non-verbal category, the majority of participants $(47 \%, n=7)$ used a single switch device. Thirteen per cent of participants in this category used the Go Talk $(n=2), 13 \%$ the Mega Wolf $(n=2)$ and $7 \%(n=1)$ the Liberator, Touch Talker, Message Mate and Wolf, respectively. With respect to the number of choices, $47 \%$ of participants who were non-verbal and used gestures used a device with one choice $(n=7), 20 \%$ used devices with two-to-eight choices $(n=3), 20 \%$ used devices with nine-to-36 choices $(n=3)$ and $13 \%$ used devices with over 37 choices $(n=2)$.

Among participants with limited verbal communication skills, $48 \%(n=12)$ used Portable Voice II with laptop, $16 \%(n=4)$ used single switch/microswitch, $8 \%(n=2)$ used Touch Talker, $8 \%(n=2)$ used Super Wolf, 4\% $(n=1)$ used Dynavox, $4 \%$ $(n=1)$ used GoTalk, 4\% $(n=1)$ used Speak Easy, $4 \%(n=1)$ used MT4 and $4 \%(n=1)$ used Wolf. The number of choices were not reported for $52 \%$ of the participants $(n=13)$ with limited verbal skills. Twenty-four per cent of this sub-group of participants $(n=6)$ used devices with nine-to-36 choices, $16 \%$ used devices with 1 choice $(n=4)$ and $8 \%$ used devices with over 37 choices $(n=2)$. The only participant with verbal skills used the Touch talker device with over 37 choices.

\section{Intervention}

Setting. Intervention was provided in a variety of settings. Intervention occurred in more than one setting for 11 studies [27-37]. The majority of studies (49\%) implemented interventions in a school setting $(n=17)$ [27, 30-32, 35, 38-48]. Thirty per cent of interventions occurred in the participants' home $(n=11)$ [27-29, 32-37, 49, 50]. Twentythree per cent of studies took place in a clinical setting such as a rehabilitation centre or therapy room $(n=8)[2,29,33,51-54]$. Fourteen per cent of studies took place in a community setting $(n=5)$ [34, 55-58]. Finally, $11 \%$ of interventions were implemented in a hospital or residential facility setting $(n=5)[28,30,31,36,59]$.

Procedures. Interventions were coded into one of three categories based upon how participants were taught to use their SGD: (a) discrete trial training, (b) milieu teaching or (c) combined instructional components. Discrete trial training involved a structured instructional plan that was characteristic of operant or behavioural teaching procedures. This type of intervention involved clear directives to the participant, prompting of correct responses, praising or reinforcing correct responding and correcting errors. Twenty-six studies utilized discrete trial training [2, 30-34, 37-39, 41-45, 51, 52, 54-58, 60].

Seven studies employed milieu teaching procedures to teach participants to use the SGD [27-29, $36,40,50,59]$. This intervention category consisted of participant-led intervention embedded in natural contexts. For example, Cosbey and Johnston [40] taught three young girls with cerebral palsy to activate a single switch SGD to request access to preferred activities or social interaction during play. Milieu teaching was provided in the participants' classroom and consisted of environmental arrangement, prompting and natural consequences (i.e. access to the requested activity).

Finally, two studies utilized interventions with combined instructional components [46, 53]. McGregor et al. [46] taught a 20-year-old male with moderate intellectual disability and cerebral palsy to use an SGD to request adult assistance or to signal task completion. Intervention consisted of pre-session discrete trials to teach the participant to use the SGD followed by milieu teaching in the individual's school.

\section{Study designs}

Study designs were classified as either experimental or non-experimental. Thirty-one of the studies were classified as experimental. Of these, 29 utilized a 
single-case design such as a multiple baseline or reversal design, to demonstrate experimental control. The remaining four studies were classified as non-experimental and implemented either an $\mathrm{AB}$ design, pre-post measures without a control group or intervention only $[27,28,36,53]$.

\section{Reliability data and treatment fidelity}

Thirty studies reported inter-observer agreement (IOA) data with respect to the dependent variable. Each of these studies reported an IOA at or above the generally accepted standard of $80 \%$ reliability [26]. Only eight studies [30, 38-40, 51, 52, 54, 60] reported treatment fidelity data for the accurate implementation of intervention procedures. The mean treatment fidelity score was $94 \%$ (range $67-$ $100 \%$ ). Two studies reported treatment fidelity data but didn't provide any other information [29, 47]. Four studies assessed treatment fidelity using an implementation checklist $[38,40,51,52]$.

\section{Outcomes}

Study outcomes were classified as positive, negative or mixed based on the data presented by the authors. Positive outcomes referred to studies in which the target communication skill(s) improved for all participants. Negative outcomes referred to studies in which none of the participants improved in the target communication skill(s). Mixed outcomes referred to studies in which some participants made improvements and others did not or in which some target skills improved and others did not. In some instances $[28,36]$ only narrative reporting of results was provided. In these cases, the classification of results was more subjective and based on the content of the narrative. Eighty-six per cent of studies $(n=30)$ reported positive outcomes and $14 \%(n=5)$ reported mixed outcomes. None of the reviewed studies reported negative outcomes.

\section{Certainty of evidence}

The certainty of evidence in each study was classified as either conclusive or inconclusive. The certainty of evidence was coded as conclusive for $54 \%(n=19)$ of the studies $[29,30,33-35,37-42,44-46,48,50$, $54,58]$. For the remaining $46 \%(n=16)$ of the studies, the certainty of evidence was coded as inconclusive due to lack of experimental design, lack of treatment effect, failure to provide sufficient detail to enable replication or the absence of reliability data.

\section{Discussion}

This systematic review of the literature yielded 35 studies investigating communication interventions using SGDs for individuals with developmental disabilities published between 1991-2009. With respect to the quantity of this literature base, it appears that substantial attention has been given to evaluating the use of SGDs to teach communication skills to individuals with developmental disabilities. In terms of the quality of this literature base, the certainty of evidence was predominately conclusive $(54 \%)$. The use of SGDs to improve communication in individuals with developmental disabilities can best be described as a promising practice [59]. Simpson et al. [59] defined a promising practice as a method of instruction that is potentially effective yet requires additional empirical investigation. Of the studies included in this review, $86 \%$ demonstrated positive outcomes. However, because some studies reporting positive outcomes were inconclusive with respect to certainty of evidence, such positive findings must be interpreted with caution. That is, the positive study outcomes should be considered in light of the study design and the study's demonstration of experimental control.

\section{Implications for practice}

The first purpose of this review was to assist practitioners in their efforts to utilize SGDs to improve the communicative ability of individuals with developmental disabilities. Implications for practice can be divided into two categories, SGD selection and SGD instruction. With respect to the selection of an SGD, practitioners may want to consider variables pertaining to the individual's current level of development and functioning as well as the targeted communication goal. The number of choices and complexity of the SGD in the studies reviewed appear to correspond to participant communication skills. Participants with the most severe communication impairments (i.e. those classified as non-verbal) were most likely to use the most basic SGD (e.g. a single switch with a single function). However, higher functioning participants whose communication goals were more complex (e.g. extending social interactions, conversational turn taking) were taught to use SGDs that were often more dynamic and versatile (i.e. capable of producing multiple phrases with multiple functions) [27, 28, 33, 41, 53, 59].

Considering future communication goals when selecting an SGD may also be important. Ideally, individuals with communication deficits will continue to develop new skills and receive on-going treatment and instruction. As progress is made, more complex goals will likely be targeted over time. 
For example, a child initially taught to request a single stimulus with a one button SGD may eventually be taught to scan an array of options on multiple buttons. Selecting an SGD that can continue to be functional for an individual whose communication skills and goals are continuing to develop and improve may reduce the need for multiple devices over time. For example, teaching a child to make a single request on a SGD capable of making multiple requests may allow for the device to continue being useful as the child targets more complex skills. This may reduce time spent teaching new skills and the cost of purchasing new SGDs. Dicarlo and Banajee [41] provide an example of this approach. In their study two young boys were taught to use an Alpha Talker to initiate social interaction. To match this goal only eight choices were provided. As the participant progressed, more choices were added to the device so that the participants communicated using between 16 and 20 choices.

In order to address issues related to instructing individuals with developmental disabilities to use SGDs, instructional strategies were classified as discrete trial training, milieu teaching or as containing elements of both approaches. Of the studies that utilized a discrete trial approach, the majority targeted requesting behaviours. However, when social initiations and conversational skills were targeted milieu teaching was more commonly used. Discrete trial training may be more practical and appropriate when teaching early communicative behaviours, such as requesting. However, when the targeted communication skill becomes more social in nature, a more naturalistic approach (i.e. milieu teaching) may be more effective. However, future research aimed at delineating the most efficient and effective approach for specific domains of communication remains warranted.

Within studies that used discrete trial training, time delay and least-to-most prompting systems were common. In addition, several studies implemented specific intervention protocols such as the picture exchange communication system (PECS) $[38,39,62]$ and functional communication training (FCT) $[49,56,58]$. The use of such interventions would seem logical as both PECS and FCT are designed to increase appropriate requesting behaviours, the most commonly targeted skill within this category. Similarly, within the category of naturalistic instruction, two of the six studies implemented the System for Augmenting Language (SAL) [63]. Under this protocol individuals were encouraged but not required to use the SGD in natural contexts. Additionally, communicative partners were trained in implementing the protocol in order to facilitate stimulus generalization for use of the SGD.
In summary, device selection seems to have been a product of desired communicative function and ease of instruction. One potential reason for this is the more sophisticated devices may also be inherently more difficult to teach participants to operate and participants with more severe disabilities may not readily acquire the necessary independence and skill with the SGD.

\section{Implications for research}

In terms of the second aim of this review, to identify areas warranting further research, there is a need for replication of these findings with additional participants. While there is a large number of studies, many had only one participant and subsequently the total participant pool is relatively small $(n=86)$. Group designs using random assignment and other appropriate controls are also needed.

Although the majority of studies reviewed here focused on improving requesting, three other communicative categories were identified including social or conversational skills, labelling items and receptive language skills. Research investigating the use of a single SGD to target multiple communicative functions is warranted. Finally, most of the studies reviewed here included SGDs that were programmed with only a few specific messages to be used in specific settings (e.g. 'I see a ball'). Such messages are often only meaningful in one setting and expanding upon these messages with many SGDs is not possible. Therefore, future research could explore how SGDs can be used across settings, such as school, work and in the community.

Declaration of interest: The authors report no conflicts of interest. The authors alone are responsible for the content and writing of the paper.

\section{References}

1. Harris SL. Your child's development. In: Powers MD, editor. Children with autism: A parents' guide. 2nd ed. Bethesda, MD: Woodbine House, Inc.; 2000. pp 155-180.

2. Sigafoos J, Drasgow E. Conditional use of aided and unaided AAC: A review and clinical case demonstration. Focus on Autism \& Other Developmental Disabilities 2001;16:152-161.

3. Lord C, Rhea P. Language and communication in autism. In: Cohen DV, editor. Handbook of autism and pervasive developmental disorders. 2nd ed. New York: John Wiley \& sons, Inc; 1997. pp 195-225.

4. Siller M, Sigmnan M. The behaviors of parents of children with autism predict the subsequent development of their children's communication. Journal of Autism and Developmental Disorders 2002;32:77-89. 
5. Lamb M, Bornstein M, Teti D. Development in infancy: An introduction. 4th ed. Mahwah, NJ: Lawrence Erlbaum Associates Publishers; 2002.

6. Sigafoos J, Arthur-Kelly M, Butterfield N. Enhancing everyday communication for children with disabilities. Baltimore, MD: Paul H Brookes Publishing Co.; 2006.

7. Beukelman DR, Mirenda P. Augmentative and alternative communication: Management of severe communication disorders in children and adults. Baltimore: Paul $\mathrm{H}$. Brookes; 1998.

8. Schlosser RW, Sigafoos J. Augmentative and alternative communication interventions for persons with developmental disabilities: Narrative review of comparative single-subject experimental studies. Research in Developmental Disabilities 2006;27:1-29.

9. Schlosser RW, Wendt O. Effects of augmentative and alternative communication intervention on speech production in children with autism: A systematic review. American Journal of Speech-Language Pathology 2008;17:212-230.

10. Bondy A, Frost L. The Picture Exchange Communication System: A training manual. Newark: Pyramid Educational Consultants; 2002.

11. Lancioni GE, O'Reilly MF, Cuvo AJ, Singh NN, Sigafoos J, Didden R. PECS and VOCAs to enable students with developmental disabilities to make requests: An overview of the literature. Research in Developmental Disabilities 2007; 28:468-488.

12. Schlosser R. Roles of speech output in augmentative and alternative communication: Narrative review. AAC: Augmentative and Alternative Communication 2003; 19:5-27.

13. Drager K, Anderson J, Debarros J, Hayes E, Liebman J, Panek E. Speech synthesis in background noise: Effects of message formulation and visual information on the intelligibility of American English DECTalk ${ }^{\mathrm{TM}}$. Augmentative and Alternative Communication 2007;23:177-186.

14. Mirenda P. Toward a functional augmentative and alternative communication for students with autism: Manual signs, graphic symbols, and voice output communication aids. Language, Speech, and Hearing Services in Schools 2003;34:203-216.

15. Schlosser RW, Lee DL. Promoting generalization and maintenance in augmentative and alternative communication: A meta-analysis of 20 years of effectiveness research. Augmentative and Alternative Communication 2000; 16:208-226.

16. Lancioni G, O'Reilly M, Basili G. Use of microswitches and speech output systems with people with severe/profound intellectual or multiple disabilities: A literature review. Research in Developmental Disabilities 2001;22:21-40.

17. Schlosser RW, Blischak DM. Is there a role for speech output in interventions for persons with autism? A review. Focus on Autism and Other Developmental Disabilities 2001; 16:170-178.

18. van der Meer LAJ, Rispoli MJ. Communication Interventions Involving Speech-Generating Devices for Children with Autism: A Review of the Literature. Developmental Neurorehabilitation 2010;13:294-306.

19. Romski M, Sevcik R, Fonseca A. Augmentative and alternative communication for persons with mental retardation. In: Abbeduto L, editor. International review of research in mental retardation: Language and communication in mental retardation. San Diego, CA: Academic Press; 2003. pp 255-280.

20. Soderholm S, Meinander M, Alantra $H$. Augmentative and alternative communication methods in locked-in syndrome. Journal of Rehabilitation Medicine 2001;33:235-239.
21. Millar D, Light J, Schlosser R. The impact of augmentative and alternative communication intervention on the speech production of individuals with developmental disabilities: A research review. Journal of Speech, Language, and Hearing Research 2006;49:248-264.

22. Schlosser RW, Sigafoos J. Navigating evidence-based information sources in augmentative and alternative communication. Augmentative \& Alternative Communication 2009; 25:225-235.

23. Simeonsson RJ, Bailey DB. Family-focused intervention: Clinical, training, and research implications. In: Marfo K, editor. Early intervention in transition: Current perspectives on programs for handicapped children. New York: England: Praeger Publishers; 1991. pp 91-108.

24. Smith NL. The certainty of judgments in health evaluations. Evaluation and Program Planning 1981;4:273-278.

25. Schlosser RW, Sigafoos J. Evidence-based communication assessment and intervention-purpose and procedures. Evidence-Based Communication Assessment and Intervention 2007;1:52-54.

26. Kennedy CH. Single-case designs for educational research. Boston: Pearson Education Inc.; 2005.

27. Adamsom LB, Romski MA, Deffebach K, Sevick RA. Symbol vocabulary and the focus of conversations: Augmenting language development for youth with mental retardation. Journal of Speech and Hearing Research 1992;35:1333-1343.

28. Cheslock MA, Barton-Hulsey A, Romski M, Sevcik RA. Using a speech-generating device to enhance communicative abilities for an adult with moderate intellectual disability. Intellectual and Developmental Disabilities 2008;46: 376-386.

29. Dattilo J, Camarata S. Facilitating conversation through selfinitiated augmentative communication treatment. Journal of Applied Behavior Analysis 1991;24:369-378.

30. Durand MV. Functional communication training using assistive devices: Recruiting natural communities of reinforcement. Journal of Applied Behavior Analysis 1999;32: 247-267.

31. Dyches TT, Davis A, Lucido BR, Young JR. Generalization of skills using pictographic and voice output communication devices. Augmentative and Alternative Communication 2002;18:124-131.

32. Lancioni GE, O'Reilly MF, Singh NN, Sigafoos J, Didden R, Oliva D, Campodonico F, de Pace C, Chiapparino C, Groeneweg J. Persons with multiple disabilities accessing stimulation and requesting social contact via microswitch and VOCA devices: New research evaluation and social validation. Research in Developmental Disabilities 2009;30:1084-1094.

33. Lund SK, Light J. The effectiveness of grammar instruction for individuals who use augmentative and alternative communication systems: A preliminary study. Journal of Speech Language \& Hearing Research 2003;46:1110-1123.

34. Schepis MM, Reid DH, Behrmann MM. Acquisition and functional use of voice output communication by persons with profound multiple disabilities. Behavior Modification 1996;20:451-468.

35. Schepis MM, Reid DH. Effects of output communication aid on interactions between support personnel and an individual with multiple disabilities. Journal of Applied Behavior Analysis 1995;28:73-77.

36. Sevcik RA, Romski MA, Adamson LB. Research directions in augmentative and alternative communication for preschool children. Disability and Rehabilitation 2004;26:1323-1329.

37. Soto G, Belfiore PJ, Acholosser RW, Haynes C. Teaching specific requests: A comparative analysis on skill acquisition and preference using two augmentative and alternative 
communication aids. Education and Training in Mental Retardation 1993;28:169-178.

38. Beck AA, Stoner JB, Bock SJ, Parton T. Comparison of PECS and the use of a VOCA: A replication. Education and Training in Developmental Disabilities 2008;43:198-216.

39. Bock SJ, Stoner JB, Beck AR, Hanley L, Prochnow J. Increasing functional communication in non-speaking preschool children: Comparison of PECS and VOCA. Education and Training in Developmental Disabilities 2005;40:264-278.

40. Cosbey JE, Johnston S. Using a single-switch voice output communication aid to increase social access for children with severe disabilities in inclusive classrooms. Research \& Practice for Persons with Severe Disabilities 2006; 31:144-156.

41. Dicarlo CF, Banajee M. Using voice output devices to increase initiations of young children with disabilities. Journal of Early Intervention 2000;23:191-199.

42. Durand MV. Functional communication training using assistive devices: Effects on challenging behavior and affect. Augmentative and Alternative Communication 1993; 9:168-176.

43. Dyches TT. Effects of switch training on the communication of children with autism and severe disabilities. Focus on Autism and other Developmental Disorders 1998; 13:151-162.

44. Lancioni GE, O'Reilly MF, Singh NN, Sigafoos J, Oliva D, Severini L. Enabling two persons with multiple disabilities to access environmental stimuli and ask for social contact through microswitches and a VOCA. Research in Developmental Disabilities 2008;29:21-28.

45. Lancioni GE, O'Reilly MF, Singh NN, Sigafoos J, Oliva D, Severini L. Three persons with multiple disabilities accessing environmental stimuli and asking for social contact through microswitch and VOCA technology. Journal of Intellectual Disability Research 2008;52:327-336.

46. McGregor G, Young J, Gerak J, Thomas B, Vogelsberg RT. Increasing functional use of an assistive communication device by a student with severe disabilities. Augmentative and Alternative Communication 1992;8:243-250.

47. Northup J, Wacker DP, Berg WK, Kelly L, Sasso G, Deraad A. The treatment of severe behavior problems in school settings using a technical assistance model. Journal of Applied Behavior Analysis 1994;27:33-47.

48. Sigafoos J, O’Reilly M, Ganz JB, Lancioni G, Schlosser RW. Supporting self-determination in AAC interventions by assessing preference for communication devices. Technology and Disability 2005;17:143-153.

49. Harding JW, Wacker, Wendy DP, et al. Analysis of multiple manding topographies during functional communication training. Educational and Treatment of Children 2009; 32:21-36.

50. O'Keefe BM, Datttilo J. Teaching the response-recode form to adults with mental retardation using AAC systems.
Augmentative and Alternative Communication 1992; 8:224-233.

51. Iacono TA, Duncum JE. Comparison of sign alone and in combination with an electronic communication device in early language intervention: Case study. Augmentative and Alternative Communication 1995;11:249-254.

52. Iacono $\mathrm{T}$, Mirenda $\mathrm{P}$, Beukelman D. Comparison of unimodal and multimodal ACC techniques for children with intellectual disabilities. Augmentative and Alternative Communication 1993;9:83-94.

53. Myers C. 'Please listen, it's my turn': Instructional approaches, curricula and contexts for supporting communication and increasing access to inclusion. Journal of Intellectual \& Developmental Disability 2007;32:263-278.

54. Winborn-Kemmerer L, Ringdahl JE, Wacker DP, Kitsukawa K. A demonstration of individual preference for novel mands during functional communication training. Journal of Applied Behavior Analysis 2009;42:185-189.

55. Mathy-Laikko P, Iacono T, Ratcliff A, Villarruel F, Yoder D, Vanderheiden G. Teaching a child with multiple disabilities to use a tactile augmentative communication device. Augmentative and Alternative Communication 1989; 5:249-256.

56. Peck SM, Wacker DP, Berg WK, et al. Choicemaking treatment of young children's severe behavior problems. Journal of Applied Behavior Analysis 1996;29:263-290.

57. Steege MW, Wacker DP, Cigrand KC, et al. Use of negative reinforcement in the treatment of self-injurious behavior. Journal of Applied Behavior Analysis 1990;23:459-467.

58. Winborn L, Wacker DP, Richman JA, Geier D. Assesment of mand selection for functional communication training packages. Journal of Applied Behavior Analysis 2002; 35:295-298.

59. Beck AR, Stoner JB, Dennis M. An investigation of aided language stimulation: Does it increase AAC use with adults with developmental disabilities and complex communication needs? Augmentative \& Alternative Communication 2009; 25:42-54.

60. Lancioni GE, O'Reilly MF, Singh NN, Sigafoos J, Oliva D, Baccani S. Teaching 'Yes' and 'No' responses to children with multiple disabilities through a program including microswitches linked to a vocal output device. Perceptual \& Motor Skills 2006;102:51-61.

61. Simpson RL, Boer-Ott SR, Griswold, D, et al. Autism spectrum disorders: Interventions and treatments for children and youth. Thousand Oaks, CA: Corwin Press; 2005.

62. Bondy A, Frost L. The Picture Exchange Communication System. Focus on Autistic Behavior 1994;9:1-19.

63. Romski MA, Sevcik RA. Developing augmented language in children with severe mental retardation. In: Warren SF, Reichle J, editors. Causes and effects in communication and language intervention. Baltimore: Paul H. Brookes; 1992. pp 113-130. 
Copyright of Developmental Neurorehabilitation is the property of Taylor \& Francis Ltd and its content may not be copied or emailed to multiple sites or posted to a listserv without the copyright holder's express written permission. However, users may print, download, or email articles for individual use. 


\section{APPENDIX D}

van der Meer, L., Sigafoos, J., O'Reilly, M., \& Lancioni, G. (2011).

Assessing preferences for AAC options in communication interventions

for individuals with developmental disabilities: A review of the literature.

Research in Developmental Disabilities, 32, 1422-1431 
Review article

\title{
Assessing preferences for AAC options in communication interventions for individuals with developmental disabilities: A review of the literature
}

\author{
Larah van der Meer $^{\mathrm{a}, *}$, Jeff Sigafoos ${ }^{\mathrm{a}}$, Mark F. O’Reilly $^{\mathrm{b}}$, Giulio E. Lancioni ${ }^{\mathrm{c}}$ \\ ${ }^{\text {a }}$ Victoria University of Wellington, New Zealand \\ ${ }^{\mathrm{b}}$ Meadows Center for Preventing Educational Risk, The University of Texas at Austin, Austin, TX, USA \\ ${ }^{\mathrm{c}}$ University of Bari, Italy
}

\section{A R T I C L E I N F O}

\section{Article history:}

Received 1 February 2011

Accepted 2 February 2011

Available online 5 March 2011

\section{Keywords:}

Augmentative and alternative

communication

Developmental disability

Preference assessment

Self-determination

\begin{abstract}
A B S T R A C T
We synthesized studies that assessed preference for using different augmentative and alternative communication (AAC) options. Studies were identified via systematic searches of electronic databases, journals, and reference lists. Studies were evaluated in terms of: (a) participants, (b) setting, (c) communication options assessed, (d) design, (e) communication skill(s) taught to the participant, (f) intervention procedures, (g) outcomes of the intervention and outcome of the preference assessment, ( $h$ ) follow-up and generalization, and (i) reliability of data collection and treatment integrity. Seven studies, involving 12 participants, met the inclusion criteria. In these studies, individuals were taught to use either speech-generating devices (SGD), (b) picture exchange (PE) systems, and/or (c) manual signs. Assessments to identify preferences for using each AAC option were conducted in each study. Sixty-seven percent $(n=8)$ of participants demonstrated some degree $(\geq 55 \%)$ of preference for using SGD compared to $33 \%(n=4)$ of participants who demonstrated some degree $(\geq 55 \%)$ of preference for PE. The results indicate that individuals with developmental disabilities often show a preference for different AAC options. Incorporating an assessment of such preferences might therefore enable individuals to exert some degree of self-determination with respect to AAC intervention. (c) 2011 Elsevier Ltd. All rights reserved.
\end{abstract}

\section{Contents}

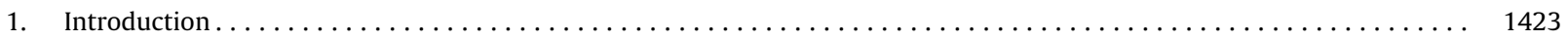

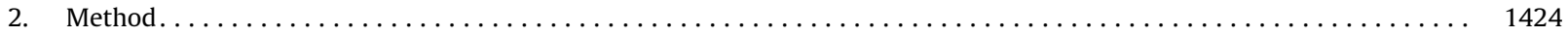

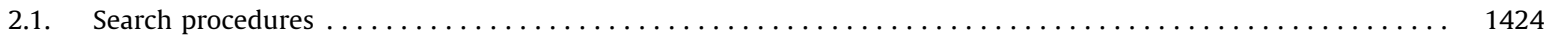

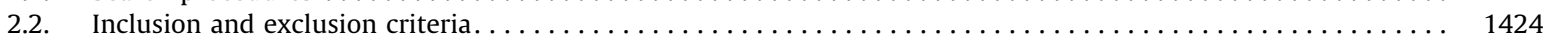

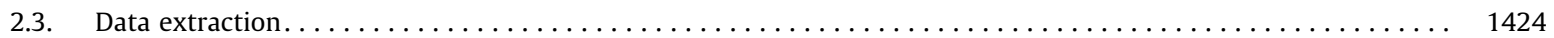

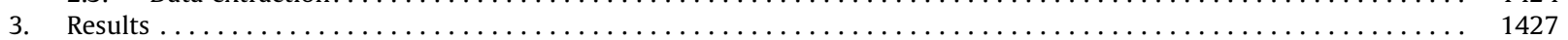

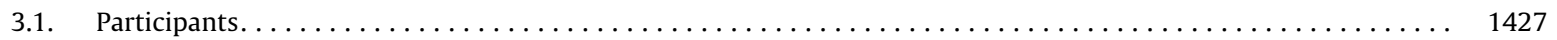

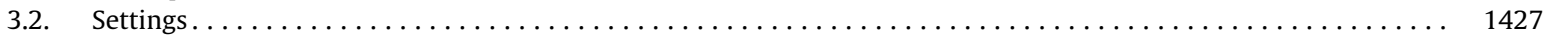

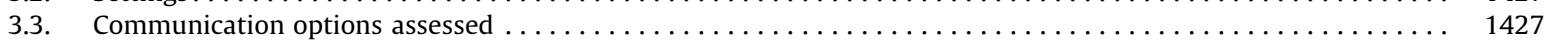

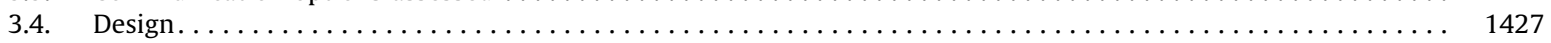

\footnotetext{
* Corresponding author at: School of Educational Psychology, Victoria University of Wellington, Karori Campus, P.O. Box 17-310, Wellington, New Zealand.

E-mail address: Larah.vanderMeer@vuw.ac.nz (L. van der Meer).
} 


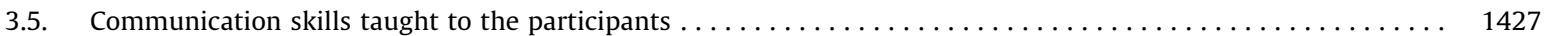

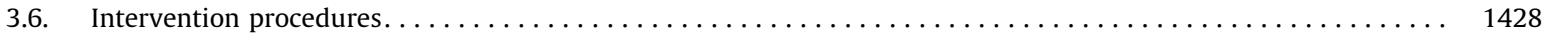

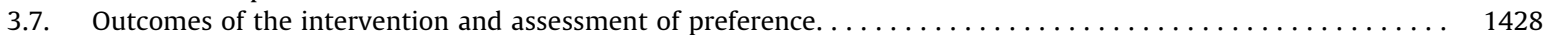

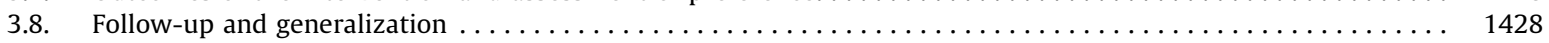

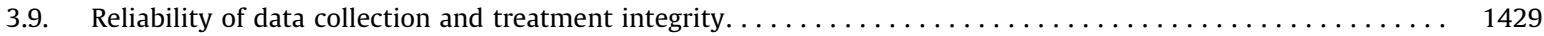

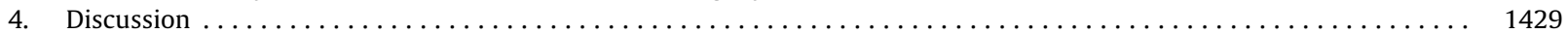

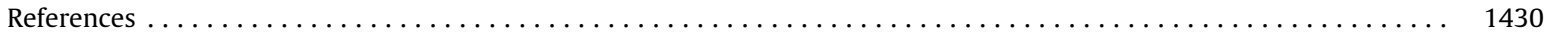

\section{Introduction}

Individuals with developmental disabilities often fail to develop speech or develop only limited speech and language abilities (National Research Council, 2001; Peeters \& Gillberg, 1999; Weitz, Dexter, \& Moore, 1997; Wing \& Attwood, 1987). Due to the prevalence and persistence of these communication deficits, individuals with developmental disabilities are often candidates for AAC, that is augmentative and alternative communication (Beukelman \& Mirenda, 2005). Various AAC options exist, which have been classified as either unaided approaches, such as gestures or manual signing; or aided systems, involving the use of external equipment to enable the person to communicate. Examples of aided AAC include low-tech communication boards (Sigafoos \& Iacono, 1993), picture-exchange (PE) systems (Bondy \& Frost, 1994; Bondy \& Frost, 2001), and speech-generating devices (SGD), often referred to as voice-output communication aids (VOCA, Lancioni et al., 2007).

When beginning an AAC intervention, Sigafoos, Drasgow, and Schlosser (2003) noted that an important decision for clinicians is the selection of a suitable AAC device for an individual. One way of doing so is to base the selection of an AAC device upon a systematic analysis of learner characteristics and environmental demands (Schlosser, Blischak, \& Koul, 2003). However, the decision is also complicated by the fact that there is considerable debate within the literature regarding which of the various unaided and aided AAC options is best suited to individuals with developmental disabilities (Mirenda, 2003; Schlosser \& Blischak, 2001).

Some guidelines have been developed to assist clinicians in selecting an appropriate AAC option to meet the communication needs of individuals with developmental disabilities (Reichle, 1991; Sigafoos \& Iacono, 1993). Once it has been established how the learner currently communicates, what vocabulary is required to meet communicative obligations and opportunities, and which communicative intents should be taught, a decision can be made regarding whether to implement an aided or unaided AAC system (Reichle, 1991). For unaided gestural mode systems it is important to consider, for example, motor skills and which vocabulary to use. For aided graphic mode systems, clinicians must decide between high (SGD) and low tech (PE) options. Sigafoos and Iacono (1993) noted several factors could be considered to help make this decision. These include: symbol option (photograph vs. line-drawing), symbol size, message representation (nature and length), message access (direct selection, scanning, eye gaze), output options (visual display, synthesized speech), expandability and portability of the device, social interaction (ability to recruit a listener, produce the communication).

Despite such guidelines, studies comparing acquisition of communication skills reveal few differences in terms of how quickly many children can learn to use some of the available AAC options. For example, PE and sign language comparison studies indicate mixed results. Some studies suggested that PE was acquired more effectively than manual signing (Adkins \& Axelrod, 2001; Rotholz \& Berkowitz, 1989), while other results indicated children acquired both PE and manual signing at an equally rapid pace (Gregory, DeLeon, \& Richman, 2009), or that acquisition varied between participants as a function of individual characteristics (Tincani, 2004). Similarly, results of studies comparing PE and SGD are also variable in terms of effectiveness of device use (Beck, Stoner, Bock, \& Parton, 2008; Bock, Stoner, Beck, Hanley, \& Prochnow, 2005).

The lack of any major differences in these comparison studies suggests how quickly an individual acquires the use of an AAC option may not be the most critical variable. Instead it is possible that the child's preference for using one AAC option over another is an important variable that could be considered when selecting AAC systems. Enabling a child to express his/ her preference could also be viewed as one way of promoting self-determination in AAC interventions (Sigafoos, 2006). Because many individuals with developmental disabilities who require AAC are unlikely to be able to verbally communicate a preference for different AAC options, it would therefore seem important to determine whether there are other ways in which individuals with developmental disabilities might indicate which of the various AAC options they would prefer to use.

Given the potential importance of assessing an individual's preference for one communication option over another on potential intervention outcomes, a review of relevant studies seems warranted. While this is a relatively new area of AAC research, with a small number of studies, a systematic review of these studies may facilitate evidence-based practice in two ways: (a) assist clinicians in their efforts to improve communication interventions for individuals with developmental disabilities, and (b) identify areas in need of future research. Specifically, this review will assess the methodology used by these studies in order to provide an initial evaluation of the viability for comparing acquisition and assessing AAC preferences in individuals with developmental disabilities. Each study included in the review was summarized and discussed in terms of: (a) participants, (b) setting, (c) communication options assessed, (d) design, (e) communication skill(s) taught to the participant, (f) intervention procedures, including procedures for assessing preferences for different AAC 
options, (g) outcomes of the intervention and assessment of preference, (h) follow-up and generalization, and (i) reliability and treatment integrity.

\section{Method}

\subsection{Search procedures}

Systematic searches were conducted in five electronic databases: Education Resources Information Center (ERIC), Medline, Linguistics and Language Behavior Abstracts (LLBA), Proquest, and PsycINFO. Publication year was not restricted, but the search was limited to English-language journal articles. Articles in other languages were excluded. The search covered all dates covered by these databases up to May 2010.

For each database, the free-text terms augmentative and alternative communication and preference were inserted into the Keywords field. Abstracts of the records returned from these electronic searches were independently reviewed by the first and second authors to identify studies for inclusion in the review (see Section 2.2). This initial search returned 48 articles, which were examined to determine if they met the criteria for inclusion in this review (see Section 2.2).

Three additional search strategies were used in order to find other possibly relevant studies that may have been missed by the electronic searches. First, the reference lists for the included studies were reviewed to identify additional articles for possible inclusion. Second, hand searches were completed for the journals that had published the included studies. Third, using an author search, the five databases were searched again for additional related work by authors of the studies that met the inclusion criteria. The reference list search yielded one additional article for possible inclusion in the current review, while the journal and author searches did not identify any further articles.

Finally, searches of the Journal of Applied Behavior Analysis (JABA), Journal of Developmental and Physical Disabilities (JDPD), and Research in Developmental Disabilities (RIDD) were undertaken because these journals have a history for including articles involving interventions that teach communication skills to individuals with developmental disabilities. The keyword preference was entered into the search section of each of these journals' websites. Two hundred and thirty nine articles were identified in the JABA search, 140 articles in the JDPD search, and 284 articles in the RIDD search. The records returned from these electronic searches were independently reviewed by the first and second authors to identify any further studies for inclusion in the review (see Section 2.2).

\subsection{Inclusion and exclusion criteria}

To be included in this review, the article had to be a research study that included individuals with a diagnosis of a developmental disability and involved a communication intervention that examined individual preferences between two or more AAC options. AAC included both unaided (e.g., gestures or manual signing) and aided (e.g., picture exchange, SGD) systems. Intervention was defined as implementing one or more therapeutic/teaching procedures for the purpose of trying to increase or improve the individual's communication skills through the use of AAC. Examples could include teaching an individual to use AAC to make requests or answer questions.

The research study had to include objective empirical data from which one could assess the success of the intervention and whether or not the individual showed a preference for using one AAC option over another. That is, the study had to include procedures for assessing whether participants showed a preference among two or more different AAC options or provide evidence related to the extent to which the participants used one AAC option more than another. Studies that focused only on the description or assessment of communication skills were not included.

Of the original 48 studies identified during the initial systematic search, five met criteria for inclusion in this review. One of the 239 articles identified in the JABA search and the article identified in the reference list search were also included, resulting in a total of seven articles for inclusion in this review. There was $100 \%$ agreement between the first and second author as to which studies were included and excluded.

\subsection{Data extraction}

All studies that met the inclusion criteria were coded by the first author in terms of: (a) participants (e.g., age, gender, number, and diagnosis), (b) setting (e.g., school, home or community setting), (c) communication options assessed (e.g., manual signing, PE, SGD), (d) design, (e) communication skill(s) taught to the participants, (f) intervention procedures (i.e., how communication skills were taught and how preference was assessed), ( $\mathrm{g}$ ) outcomes of the intervention and assessment of preference, ( $h$ ) follow-up and generalization, if any, and (i) reliability of data collection and treatment integrity. The results of the studies were analyzed to determine the magnitude of preference for one communication option over another.

The first author then wrote a summary of each of the seven included studies (see Table 1 ) with the accuracy of these summaries assessed by the second author using a checklist that included the initial table with summaries of the studies and a number of questions regarding various details of the study (i.e., Is this an accurate description of the purpose of the study?, Is this an accurate description of the participants?, Is this an accurate description of the communication options assessed?, Is this an accurate description of the design?, and Is this an accurate description of the outcomes of 
Table 1

Studies assessing preference for various communication options.

\begin{tabular}{|c|c|c|c|c|c|c|}
\hline Study & Purpose & Participants & $\begin{array}{l}\text { Communication } \\
\text { options assessed }\end{array}$ & Design & Outcomes & $\begin{array}{l}\text { Magnitude of preference } \\
\text { (selections/opportunities) }\end{array}$ \\
\hline $\begin{array}{l}\text { Cannella-Malone } \\
\text { et al. (2009) }\end{array}$ & $\begin{array}{l}\text { To compare acquisition of } \\
\text { requesting for specific items } \\
\text { and preference between } 2 \text { SGD } \\
\text { and a PE system. To teach } \\
\text { functional use of the preferred } \\
\text { communication option. }\end{array}$ & $\begin{array}{l}2 \text { males, } 1 \text { with } \\
\text { mitochondrial disorder } \\
\text { and significant } \\
\text { intellectual disability (13 } \\
\text { years) and } 1 \text { with } \\
\text { neurological disorder } \\
\text { etiology-not-specified, } \\
\text { seizure disorder, and } \\
\text { significant intellectual } \\
\text { disability ( } 11 \text { years) }\end{array}$ & $\begin{array}{l}\text { Picture } \\
\text { communication board; } \\
\text { Mini-messageMate } \\
\text { (digitized) and Cyrano } \\
\text { Communicator } \\
\text { (synthesized) SGD. } \\
\text { Picture icons used for } \\
\text { all } 3 \text { AAC devices were } \\
\text { digital color } \\
\text { photographs }\end{array}$ & $\begin{array}{l}\text { Multiple-probe- } \\
\text { across-AAC-devices } \\
\text { design, with a } \\
\text { changing-criterion } \\
\text { design during } \\
\text { functional training }\end{array}$ & $\begin{array}{l}\text { Participant A only learnt } \\
\text { to use the PE system to } \\
\text { request preferred items; } \\
\text { therefore did not } \\
\text { commence to the } \\
\text { preference assessment or } \\
\text { functional training. } \\
\text { Participant B learnt to use } \\
\text { all three communication } \\
\text { options and demonstrated } \\
\text { a preference for the } \\
\text { Cyrano Communicator. } \\
\text { Reached criterion for } \\
\text { functional training }\end{array}$ & $\begin{array}{l}\text { Cyrano Communicator } \\
\text { selected } 65 / 78 \\
\text { opportunities (83\%) }\end{array}$ \\
\hline $\begin{array}{l}\text { Iacono and } \\
\text { Duncum (1995) }\end{array}$ & $\begin{array}{l}\text { To compare the use of sign alone } \\
\text { and sign in combination with a } \\
\text { SGD on the development of } \\
\text { expressive language skills (with } \\
\text { vocabulary targeting pretend } \\
\text { cooking and dressing-up scripts) }\end{array}$ & $\begin{array}{l}1 \text { female with Down } \\
\text { syndrome ( } 2 \text { years, } 8 \\
\text { months) }\end{array}$ & $\begin{array}{l}\text { Signs from the } \\
\text { Dictionary of } \\
\text { Australasian Signs } \\
\text { (Jeanes et al., 1989); } \\
\text { DynaVox }{ }^{\mathbb{B}} \text { SGD (voice- } \\
\text { output not specified), } \\
\text { with DynaSims }{ }^{\mathbb{B}} \\
\text { symbols }\end{array}$ & $\begin{array}{l}\text { Alternating- } \\
\text { treatments design }\end{array}$ & $\begin{array}{l}\text { Overall preference for the } \\
\text { sign and SGD condition for } \\
\text { both spontaneous/ } \\
\text { response and imitated } \\
\text { productions. More single- } \\
\text { word and two- and three- } \\
\text { word phrases in sign and } \\
\text { SGD condition compared } \\
\text { to sign alone condition }\end{array}$ & $\begin{array}{l}\text { SGD identified by more } \\
\text { spontaneous/response } \\
\text { productions than the sign } \\
\text { alone condition }\end{array}$ \\
\hline Sigafoos et al. (2009) & $\begin{array}{l}\text { To compare the effects of } \\
\text { acquisition (study } 1 \text { ) and } \\
\text { preference (study } 2 \text { ) for a SGD- } \\
\text { vs. PE-based requesting } \\
\text { response; and the effects on } \\
\text { social interaction (study 3) }\end{array}$ & $\begin{array}{l}1 \text { male with Down } \\
\text { syndrome and Autistic } \\
\text { disorder ( } 15 \text { years) }\end{array}$ & $\begin{array}{l}\text { PE system; Tech/Talk } \\
68^{\mathrm{TM}} \text { SGD (digitized). } \\
\text { Both AAC devices used } \\
\text { Picture } \\
\text { Communication } \\
\text { Symbols }^{\mathrm{TM}} \text { (Mayer- } \\
\text { Johnson Co, 1994) }\end{array}$ & $\begin{array}{l}\text { Alternating- } \\
\text { treatments design }\end{array}$ & $\begin{array}{l}\text { Equally rapid acquisition } \\
\text { of PE- and SGD-based } \\
\text { requesting response. } \\
\text { Slight preference for PE } \\
\text { (56\%) over the SGD (44\%). } \\
\text { Distancing manipulation } \\
\text { resulted in significant } \\
\text { reductions in social } \\
\text { withdrawal }\end{array}$ & PE 35/62 (56\%) \\
\hline Sigafoos et al. (2005) & $\begin{array}{l}\text { To compare acquisition of } \\
\text { requesting more (i.e., "I want } \\
\text { more") snacks and preference } \\
\text { between } 3 \text { SGD (demonstration } \\
\text { 1). To compare acquisition of } \\
\text { requesting specific snack items } \\
\text { and preference between the SGD } \\
\text { chosen in demonstration } 1 \text { and a } \\
\text { PE system (demonstration } 2 \text { ) }\end{array}$ & $\begin{array}{l}2 \text { males, } 1 \text { with autism (12 } \\
\text { years) and } 1 \text { with } \\
\text { intellectual disability ( } 16 \\
\text { years) }\end{array}$ & $\begin{array}{l}\text { BIGmack }{ }^{\mathrm{TM}} \text { switch, } \\
\text { Tech/Talk 6/8 }{ }^{\mathrm{TM}} \text {, and } \\
\text { Mini-messageMate } \\
\text { (Words+) SGD (all } \\
\text { digitized); Picture } \\
\text { communication board. } \\
\text { All AAC devices used } \\
\text { Picture } \\
\text { Communication } \\
\text { Symbols }{ }^{\mathrm{TM}} \text { (Mayer- } \\
\text { Johnson Co, 1994) }\end{array}$ & $\begin{array}{l}\text { Multiple-baseline } \\
\text { design }\end{array}$ & $\begin{array}{l}\text { Both participants learnt to } \\
\text { use all three SGD and the } \\
\text { communication board to } \\
\text { make requests. } 1 \\
\text { participant preferred the } \\
\text { Mini-messageMate, while } \\
\text { the other preferred the } \\
\text { Tech/Talk. In the second } \\
\text { demonstration both } \\
\text { participants preferred the } \\
\text { SGD (100\% and } 70 \% \text { ) over } \\
\text { the PE-based system }\end{array}$ & $\begin{array}{l}\text { Demonstration } 1 \\
\text { Jason = Tech/Talk } \\
\text { Ryan = Mini- } \\
\text { messageMate } \\
\text { Demonstration } 2 \\
\text { Jason = Tech/Talk 22/31 } \\
\text { (70\%) Ryan = Mini- } \\
\text { messageMate 6/6 } \\
\text { opportunities (100\%) }\end{array}$ \\
\hline
\end{tabular}




\section{Table 1 (Continued)}

\begin{tabular}{|c|c|c|c|c|c|c|}
\hline Study & Purpose & Participants & $\begin{array}{l}\text { Communication } \\
\text { options assessed }\end{array}$ & Design & Outcomes & $\begin{array}{l}\text { Magnitude of preference } \\
\text { (selections/opportunities) }\end{array}$ \\
\hline Son et al. (2006) & $\begin{array}{l}\text { To compare acquisition of } \\
\text { requesting for specific items and } \\
\text { preference for a SGD vs. PE } \\
\text { system }\end{array}$ & $\begin{array}{l}2 \text { females with autism } \\
\text { ( } 5 \text { years, } 5 \text { months; } \\
3 \text { years, } 8 \text { months) and } 1 \\
\text { male with pervasive } \\
\text { developmental disorder } \\
\text { ( } 3 \text { years) }\end{array}$ & $\begin{array}{l}\text { PE system; Tech/Talk } \\
68^{\mathrm{TM}} \text { SGD (digitized). } \\
\text { Both AAC devices used } \\
\text { Picture } \\
\text { Communication } \\
\text { Symbols }{ }^{\mathrm{TM}} \text { (Mayer- } \\
\text { Johnson Co, 1994) }\end{array}$ & $\begin{array}{l}\text { Alternating- } \\
\text { treatments design }\end{array}$ & $\begin{array}{l}\text { Little difference in } \\
\text { acquisition of PE- and } \\
\text { SGD-based requesting } \\
\text { response. Two } \\
\text { participants displayed } \\
\text { preference for PE ( } 98 \% \text { and } \\
72 \% \text { ), while the third } \\
\text { demonstrated a } \\
\text { preference for the SGD } \\
\text { (94\%). All children used } \\
\text { their preferred device } \\
\text { with a high level of } \\
\text { proficiency }(86-100 \%)\end{array}$ & $\begin{array}{l}\text { Kim }=\text { SGD 30/32 (94\%) } \\
\text { Lucy }=\text { PE 86/88 (98\%) } \\
\text { Bruce = PE 52/72 (72\%) }\end{array}$ \\
\hline Soto et al. (1993) & $\begin{array}{l}\text { To compare acquisition of } \\
\text { requesting for specific items } \\
\text { (surrounding drawing and } \\
\text { snack-time activities) and } \\
\text { preference for a SGD vs. PE } \\
\text { system in two settings. Assess } \\
\text { generalization and maintenance } \\
\text { of requesting skills with the } \\
\text { preferred AAC device }\end{array}$ & $\begin{array}{l}1 \text { male with severe to } \\
\text { profound intellectual } \\
\text { disability ( } 22 \text { years) }\end{array}$ & $\begin{array}{l}\text { Picture } \\
\text { communication board; } \\
\text { Wolf }^{\mathrm{TM}} \text { SGD (voice- } \\
\text { output not specified). } \\
\text { Both AAC devices used } \\
\text { Sigsymbols (Cregan, } \\
\text { 1984; Cregan \& Llyod, } \\
\text { 1990) }\end{array}$ & $\begin{array}{l}\text { Multiple-baseline } \\
\text { design across settings } \\
\text { with alternating } \\
\text { treatments }\end{array}$ & $\begin{array}{l}\text { The participant reached } \\
\text { criterion in both settings } \\
\text { for the SGD and PE } \\
\text { systems at similar rates. } \\
\text { Demonstrated preference } \\
\text { for the SGD } 100 \% \text { of the } \\
\text { time, with } 90-100 \% \\
\text { correct requesting. } \\
\text { Generalization and } \\
\text { maintenance } \\
\text { demonstrated }\end{array}$ & $\begin{array}{l}\text { SGD } 100 \% \text { Amount of } \\
\text { opportunities not } \\
\text { specified }\end{array}$ \\
\hline $\begin{array}{l}\text { Winborn-Kemmerer } \\
\text { et al. (2009) }\end{array}$ & $\begin{array}{l}\text { To assess the effects of FCT to } \\
\text { activate a SGD and touch a } \\
\text { picture card to gain access to } \\
\text { attention and tangible items in } \\
\text { order to reduce challenging } \\
\text { behavior; and assess preference } \\
\text { for one AAC option over the other }\end{array}$ & $\begin{array}{l}1 \text { male with pervasive } \\
\text { developmental delays and } \\
\text { seizure disorder ( } 7 \text { years) } \\
\text { and } 1 \text { female with } \\
\text { intellectual disability ( } 20 \\
\text { years) }\end{array}$ & $\begin{array}{l}\text { Picture } \\
\text { communication } \\
\text { system (picture of } \\
\text { therapist and an item); } \\
\text { BIGmack }{ }^{\mathrm{TM}} \text { SGD } \\
\text { (digitized message: } \\
\text { "Play, please" or "Can I } \\
\text { have my --- } \\
\text { please?"). }\end{array}$ & $\begin{array}{l}\text { ABAB design } \\
(A=\text { picture card, } \\
B=S G D) \text {, and } \\
\text { multielement design } \\
\text { where training } \\
\text { procedures were } \\
\text { randomly drawn (i.e., } \\
\text { picture card or SGD), } \\
\text { followed by the } \\
\text { alternate condition } \\
\text { and another random } \\
\text { draw }\end{array}$ & $\begin{array}{l}\text { Problem behavior and } \\
\text { correct requesting } \\
\text { increased for both } \\
\text { participants as a function } \\
\text { of the intervention. The } \\
\text { choice analysis } \\
\text { demonstrated that the } \\
\text { male participant preferred } \\
\text { the picture card, while the } \\
\text { female preferred the SGD } \\
\text { indicated by more } \\
\text { responding }\end{array}$ & $\begin{array}{l}\text { Jack }=\text { PE indicated by } \\
\text { more }(M=0.6) \text { responses } \\
\text { per minute than SGD } \\
(M=0.2) \text { Sally }=\text { SGD } \\
\text { indicated by more } \\
(M=0.6) \text { responses per } \\
\text { minute than PE }(M=0.2)\end{array}$ \\
\hline
\end{tabular}


the intervention?). There were 35 items on which there could be agreement or disagreement regarding data extraction and summary (i.e., 7 studies with 5 questions per study). Agreement was obtained on 100\% of the items.

\section{Results}

A total of 10 interventions/experiments were reported in the seven included studies. Table 1 summarizes the purpose, participants, communication options assessed, design, outcomes, and magnitude of preference for each of the seven included studies.

\subsection{Participants}

A total of 12 participants ( 8 males, 4 females) were included in the studies. Participants were reported as having a range of disabilities, including intellectual disability $(n=3,25 \%)$, autism $(n=3,25 \%)$, Down syndrome $(n=2,16.7 \%)$, pervasive developmental disorder $(n=2,16.7 \%)$, mitochondrial disorder $(n=1,8.3 \%)$, and neurological disorder etiology-not-specified ( $n=1,8.3 \%)$. Four (33.3\%) of these participants also had a combination of diagnoses (e.g., intellectual disability and autism).

Ages ranged from 2.8 to 22 years (mean=10.9). Sample sizes from the seven studies ranged from one to three participants. Only one study (Son, Sigafoos, O’Reilly, \& Lancioni, 2006) had three participants. Three (42.9\%) studies had one participant and the remaining three (42.9\%) studies had two participants.

\subsection{Settings}

Forty-three percent $(n=3)$ of the interventions were undertaken in the participants' schools. Two (28.6\%) studies were undertaken in a clinical setting. One (14.3\%) study took place in the participant's home and the final study (Soto, Belfiore, Schlosser, \& Haynes, 1993) was undertaken at the participant's group home and vocational centre, with generalization probes conducted in a community setting (i.e., fast food restaurant).

\subsection{Communication options assessed}

Three different communication options were assessed in the 10 individual interventions reported: SGD, PE, and manual signing. A total of six different types of SGD were used. Of the various SGD used, the Tech/Talk 6 X8 ${ }^{\mathrm{TM}}$ had the highest frequency $(n=3,50 \%)$, both the Mini-messageMate ${ }^{\mathrm{TM}}$ and BIGmack $^{\mathrm{TM}}$ were used twice (33.3\%), and the Cyrano communicator, DynaVox ${ }^{\circledR}$ and Wolf ${ }^{\mathrm{TM}}$ were all used once. Four different types of symbols were used to represent messages on both the PE systems and SGD. Of these, the Picture Communication Symbols ${ }^{\mathrm{TM}}$ (Mayer-Johnson Co, 1994) had the highest frequency $(n=3,75 \%)$. Digital photographs, DynaSims ${ }^{\circledR}$, and Sigsymbols (Cregan, 1984; Cregan \& Llyod, 1990) were each used once. One study (Winborn-Kemmerer, Ringdahl, Wacker, \& Kitsukawa, 2009) did not specify what symbols were used to represent the messages used on the SGD and PE-based systems. The one study (Iacono \& Duncum, 1995) comparing manual signs and a SGD used signs from the Dictionary of Australasian Signs (Jeanes, Reynolds, \& Coleman, 1989).

The majority of studies $(n=6,86 \%)$ compared how efficiently and effectively participants learnt to use SGD and PE systems. They also assessed which of these AAC systems participants preferred to use (see Section 3.6). Only one study (Iacono \& Duncum, 1995) compared the use of manual signing with a SGD. Of the studies that included SGD, two studies taught participants to use two (Cannella-Malone, DeBar, \& Sigafoos, 2009) or three (Sigafoos, O’Reilly, Ganz, Lancioni, \& Schlosser, 2005) different types of SGD to request access to preferred objects. Sigafoos et al. (2005) assessed which of these SGD participants preferred to use and continued intervention with the participants' preferred SGD, while simultaneously teaching the participant to use a PE system. They then assessed whether the participants preferred to use the SGD or the PE system.

\subsection{Design}

All of the studies included in the current review used some type of single-case experimental design (Kennedy, 2005) to evaluate the effects of the intervention on communication using AAC devices. Specifically, all studies implemented baseline and intervention phases using some variation of a multiple-baseline and/or alternating-treatments design. Following intervention, six (86\%) of the studies implemented a choice assessment phase to determine which AAC option the participant preferred to use (see Section 3.6). The remaining study (Iacono \& Duncum, 1995) assessed preference in terms of effectiveness of AAC device use.

\subsection{Communication skills taught to the participants}

The majority of studies ( $n=6,86 \%$ ) taught participants some form of requesting as the primary communication skill. Fifty percent $(n=3)$ of these six studies (Son et al., 2006; Soto et al., 1993; Winborn-Kemmerer et al., 2009) implemented interventions targeting requesting of specific preferred items (e.g., "I want chocolate, please"). One of these six studies (Sigafoos et al., 2009) taught the participants to make generalized requests for snacks (e.g., "I want a snack, please"). The 
remaining two studies (Cannella-Malone et al., 2009; Sigafoos et al., 2005) first taught participants to make a general request for preferred items and then taught the participant to request specific items (e.g., "I want a cookie." "I want a potato chip."). Two of these studies (Cannella-Malone et al., 2009; Sigafoos et al., 2005) also implemented an intervention to teach the participant how to turn on the SGD. One study (Iacono \& Duncum, 1995) taught the sole participant expressive language skills (i.e., single word vocabulary, as well as two- and three-word phrases).

\subsection{Intervention procedures}

Eighty-six percent $(n=6)$ of the studies used systematic instructional procedures to teach communication skills. These procedures included (a) presenting an opportunity or discriminative stimulus, (b) prompting a communicative behavior, (c) fading prompts, and (d) providing reinforcement for correct communicative behavior. Within this broad class of procedures, some studies also identified more specific techniques as their core teaching mechanism, including (a) functional communication training (FCT, Winborn-Kemmerer et al., 2009), (b) graduated guidance (Sigafoos et al., 2009), and (c) leastto-most prompting (Sigafoos et al., 2005; Son et al., 2006; Soto et al., 1993).

Following intervention, these six studies implemented a structured choice making arrangement (e.g., Sigafoos, 1998) to identify a participant's preference for one AAC option over another. The general approach used by these studies involved making all communication options (i.e., both the SGD and PE system) available and then allowing the participant to choose one device. The remaining device/s were removed and a requesting opportunity was initiated with the chosen AAC option. When the participant consistently chose one option over another, it was considered to be his/her preferred mode of communication (see Section 3.7).

One study (Iacono \& Duncum, 1995) implemented a child-directed approach. Intervention centered around activities that the participant had demonstrated an interest in, including pretend cooking and dressing up. Single-word vocabulary related to these activities was targeted for intervention. The treatment procedure involved providing models in response to the child's activities and initiations (e.g., "hat" when the participant put a hat on a doll) and specific reinforcement (e.g., "that's right") if the participant imitated the model. This was followed by a two-word expansion. The trainer's models varied according to the treatment condition (sign or sign and SGD). A post-intervention phase was implemented with the most effective (in terms of word production) treatment modality, which was considered to be the participant's preferred AAC option.

\subsection{Outcomes of the intervention and assessment of preference}

Outcomes for AAC device acquisition were classified and ranked into one of three outcome categories: (a) positive outcomes in which target communication skill(s) improved for all participants, (b) negative outcomes in which none of the participants improved in the target communication skill(s), and finally (c) mixed outcomes in which improvement was evident for some, but not all participants in the study, or in which some target skills improved and others did not.

Eighty-six percent $(n=6)$ of studies reported positive outcomes in terms of participants learning to use the AAC devices for targeted communication skills. Only one study (Cannella-Malone et al., 2009) reported mixed outcomes, where one of the two participants did not reach criterion for AAC device acquisition, and therefore did not progress to the preference assessment phase of the study. No studies reported negative outcomes.

For the purpose of the current review a communication option was classified as highly preferred if it was selected on at least $70 \%$ of the provided opportunities, moderately preferred if it was selected on $55-70 \%$ of opportunities, and nonpreferred if it was selected on less than 55\% of the opportunities. For the 6 studies, involving 10 participants, that compared preference for SGD vs. PE, $50 \%$ of the participants $(n=5)$ demonstrated a high preference for the SGD over PE. This included the study that first assessed preference between three SGD and then assessed preference between the preferred SGD and a PE system (Sigafoos et al., 2005). Thirty percent $(n=3)$ of the participants demonstrated a high preference for PE. One study (Winborn-Kemmerer et al., 2009) assessed preference in terms of amount of responses made with each AAC device per minute. For this study one participant exhibited a preference for the SGD, while the other participant preferred the PE system. The one study (Iacono \& Duncum, 1995) that compared the combined use of signs and a SGD and signs alone found that the participant showed a preference for using the SGD, but the participant also showed an increased production of single-word vocabulary as well as two- and three-word phrases during the combined sign and SGD condition.

When comparing all three communication options (SGD, PE, and manual signing) across the seven studies included in this review, $67 \%(n=8)$ of the participants demonstrated some degree of preference $(\geq 55 \%)$ for using a SGD. Thirty-three percent $(n=4)$ of the participants, in contrast, demonstrated some degree of preference $(\geq 55 \%)$ for PE.

\subsection{Follow-up and generalization}

One study (Soto et al., 1993) undertook follow-up and generalization probes. Only the preferred AAC option (SGD) was used during follow-up and generalization. Communication skills maintained at a level of 66-100\% correct requesting for four weeks. SGD use also generalized to different settings and different communication partners with $70-$ $75 \%$ correct requesting. 


\subsection{Reliability of data collection and treatment integrity}

All of the studies included checks on the reliability of data collection with respect to the dependent variables, that is interobserver agreement (IOA). Each of these studies reported average rates of IOA above the generally accepted standard of $80 \%$ (Kennedy, 2005). Fewer studies $(57 \%, n=4)$ reported treatment integrity data for the accurate implementation of intervention procedures. All of these studies reported high treatment integrity scores of over $80 \%$ correct implementation. One of these four studies (Sigafoos et al., 2009) did not collect treatment integrity data for one of the three demonstrations within their study.

\section{Discussion}

This systematic search identified 10 interventions reported in 7 studies published between 1993 and 2009 . Five of these seven studies have been published since 2005, indicating an increase in assessing individuals' preferences between different modes of AAC and the potential impacts on intervention outcomes. In terms of quality of the evidence, all of the studies utilized appropriate single-case experimental designs with generally positive outcomes with respect to teaching requesting skills using each of the communication options assessed.

A key aspect of these studies was the attempt to assess preference for different AAC options. Structured choice-making arrangements (e.g., Sigafoos, 1998) were utilized to assess preferences for one mode communication over another. Following the initial AAC intervention to teach requesting skills, the general approach used by the studies synthesized in this review was to give the individual an opportunity to choose which of the AAC options (SGD or PE) to use. When the individual consistently chose one option over another, it was considered to be his/her preferred mode of communication. Using this approach, it appeared that most participants did in fact appear to show some degree of preference for one AAC option over another. Because many AAC options exist and because it is difficult to predetermine whether any given option is viable, the preference assessment approach described in this review maybe one way to enable individuals with developmental disabilities to exert some control over their lives and participate in this important clinical decision.

However, results with respect to the certainty of evidence in terms of an individual's preference, or the 'magnitude' of preference, for one AAC option over another should be interpreted with caution. The literature pertaining to general choicemaking and assessment of preference in individuals with developmental disabilities (Sigafoos, 1998) defines preference in behavioral terms specifically as the frequency with which one selects an object or activity from an array of options. It is assumed that objects or activities selected more frequently are more preferred than objects or activities selected less frequently. The choice-making paradigm used in the studies included in this review could be seen as a type of preference assessment similar to those that have been used to identify stimuli for use as reinforcers (Cannella, O'Reilly, \& Lancioni, 2005). Generally this preference assessment literature defines those alternatives that are selected or approached $80 \%$ of the time as preferred (Green et al., 2008; Pace, Ivancic, Edwards, Iwata, \& Page, 1985; Stafford, Alberto, Fredrick, Heflin, \& Heller, 2002). However, Son et al. (2006) defined an AAC option as preferred if it was selected on $70 \%$ or more of the total choice opportunities. Therefore, as mentioned, for the purpose of the current review, a communication option was also classified as highly preferred if it was selected on at least $70 \%$ of opportunities.

An important practical issue is how many choice opportunities are required to enable the demonstration of a preference for one mode of communication over another? In the studies analyzed in the current review, participants were given between 6 and 88 choice opportunities. This is similar to other preference assessment research (Green et al., 2008; Pace et al., 1985; Stafford et al., 2002) where the range of choice opportunities has also varied greatly. With such disparity in the amount of opportunities individuals were given to choose one stimulus over another, we cannot reliably define a percentage of selections for which an item can be considered preferred.

Similar to previous research (Beck et al., 2008; Bock et al., 2005), in the six studies that utilized a direct choice assessment (Cannella-Malone et al., 2009; Sigafoos et al., 2009; Sigafoos et al., 2005; Son et al., 2006; Soto et al., 1993; WinbornKemmerer et al., 2009) participants gained proficiency in using each AAC system relatively quickly and with comparable ease. Because participants had equal exposure to each device prior to assessing their preferences, they could be considered to be capable of making an informed choice when demonstrating preference for one mode of communication over another and that preference was generally not due to differences in performance between AAC options. However, it remains unclear why these individuals preferred one option over another. Son et al. (2006) and Sigafoos et al. (2005) suggest that there is perhaps something about the devices themselves (shape, color, size, voice-output) or the way that they are used (pressing a switch on a SGD or handing over a card for PE) that makes them more or less appealing. Future research could investigate several such variables that might influence AAC preferences.

It is widely documented in the choice assessment literature that preferences change over time (e.g., Stafford et al., 2002) and may need to be reassessed at regular intervals. This would seem necessary not only because giving an individual the opportunity to express preferences is important in its own right, but because it may also affect communication intervention outcomes.

The studies examined in this review do not provide any results in terms of the effect preference may have on potential intervention progress and outcomes. In each of these studies, the child's preference for AAC options was assessed post hoc; that is, the choice-making preference assessment was undertaken only after learning to use both options. This post hoc assessment of preferences prevents an evaluation of the effects of preference on the child's progress during the 
communication intervention. It is unclear, however, whether these preferences can be identified during, and incorporated into, the beginning stages of intervention. Only one of the studies in this review assessed maintenance and generalization of communication skills with the preferred AAC device (Soto et al., 1993). For the most part, it therefore remains unclear whether utilizing preferred AAC options actually improves intervention outcomes in terms of promoting long-term maintenance of newly acquired communication skills. This limitation is important, as it is clear that individuals often abandon the use of AAC (Schlosser \& Lee, 2000). Findings indicating improved progress during intervention as well as better maintenance and generalization of communication skills with the preferred device would provide further evidence for practitioners to allow individuals themselves to determine which communication option they would rather use.

Because this is an emerging field of AAC with a relatively small corpus of research, it is evident that the studies evaluated within this review are limited in several ways and that replications are warranted to address these limitations. Firstly, although the participant sample covered a good range of ages, diagnoses, and intervention contexts, the number is still limited with only 12 participants across the 7 studies. It therefore remains unclear what percentage of individuals with developmental disabilities will in fact show a preference for one mode of communication over another.

Secondly, in six of the seven studies just two AAC options (SGD vs. PE) have been compared. Only one study (Iacono \& Duncum, 1995) compared manual signing and use of an SGD. Within the current sample the majority (67\%) of participants demonstrated some degree of preference ( $\geq 55 \%$ ) for SGD. These results reflect recent reviews (Rispoli, Franco, van der Meer, Lang, \& Carmargo, 2010; van der Meer \& Rispoli, 2010) providing further evidence for the effectiveness of SGD-based communication interventions. However, preference for other AAC options, such as manual signing and PE or comparison of SGD, PE, and manual signing, has not yet been investigated. It is therefore uncertain whether most children do in fact prefer SGD over other communication options.

Third, in six of the seven studies the only dependent variable studied has been requesting behavior. Iacono and Duncum (1995) taught expressive language skills and Winborn-Kemmerer et al. (2009) taught requesting in terms of FCT. Through FCT participants were taught appropriate communicative acts (to request a preferred item or to play) to replace their respective challenging behaviors. However, extension to more social and complex communicative interactions needs to be investigated. It remains unclear whether preference will influence how well the individual learns new and more advanced communication skills (e.g., commenting on the environment, initiating and maintaining conversations, and answering questions).

Finally, these studies have not undertaken a comprehensive assessment of the individual's skills and deficits to investigate which factors may influence AAC system acquisition and preference. It can be difficult for clinicians to determine the most appropriate mode of communication for an individual (Sigafoos \& Drasgow, 2001). Assessing learner characteristics, such as communicative and motor skills level, may predict not only which AAC modality the individual will be most effective at learning to use, but also which option he or she will prefer to use. For example, do children with more functional communication skills choose a more complex AAC device, such as a SGD? If clear patterns between learner characteristics, acquisition, and preference do emerge then selection of an appropriate AAC system for individuals with developmental disabilities will be much easier to determine. Systematic replications to address these limitations would lead to further understanding on whether and how preferences will influence the effectiveness of AAC interventions.

It would also be important to determine whether preference for different AAC options might vary across situations and communicative partners. An SGD might, for example, be preferred for use in community settings, whereas manual signs might be preferred when communicating with peers who know manual signs. Such factors were not investigated in the current studies reviewed. Future research should therefore assess whether preferences for different AAC options generalizes across situations and different communicative partners.

The current review has evaluated the feasibility and effects of incorporating aspects of self-determination into communication interventions for individuals with developmental disabilities. Specifically it has assessed seven studies from an emerging field of AAC research incorporating a promising methodology to evaluate individuals' preferences for using one communication option over another. Findings suggest that while individuals are able to be taught to use alternative communication options (SGD, PE, and manual signing) for requesting, and often demonstrate a preference for one communication option over another, the evidence base supporting these findings are limited and the numerous variables that might influence $A A C$ preferences and use require future research.

\section{Conflict of interest}

The authors report no conflicts of interests. The authors alone are responsible for content and writing of the paper.

\section{References}

Adkins, T., \& Axelrod, S. (2001). Topography- versus selection-based responding: Comparison of mand acquisition in each modality. The Behavior Analyst Today, 2, 259-266.

Beck, A. R., Stoner, J. B., Bock, S. J., \& Parton, T. (2008). Comparison of PECS and the use of a VOCA: A replication. Education and Training in Developmental Disabilities, 43, 198-216.

Beukelman, D., \& Mirenda, P. (2005). Augmentative and alternative communication: Supporting children and adults with complex communication needs (3rd ed.). Baltimore: Paul H Brookes Publishing Co.

Bock, S. J., Stoner, J. B., Beck, A. R., Hanley, L., \& Prochnow, J. (2005). Increasing functional communication in non-speaking preschool children: Comparison of PECS and VOCA. Education and Training in Developmental Disabilities, 40, 268-278. 
Bondy, A., \& Frost, L. (1994). The picture exchange communication system. Focus on Autistic Behavior, 9, 1-19.

Bondy, A., \& Frost, L. (2001). The picture exchange communication system. Behavior Modification, 25, 725-744.

Cannella, H., O'Reilly, M., \& Lancioni, G. (2005). Choice and preference assessment research with people with severe to profound developmental disabilities: A review of the literature. Research in Developmental Disabilities, 26, 1-15.

Cannella-Malone, H., DeBar, R., \& Sigafoos, J. (2009). An examination of preference for augmentative and alternative communication devices with two boys with significant intellectual disabilities. Augmentative and Alternative Communication, 25, 262-273.

Cregan, A. (1984, January). Sigsymbols-A graphic aid to communication and language development. Paper presented at the Curriculum Conference. Cambridge, England: Cambridge Institute of Education.

Cregan, A., \& Llyod, L. (1990). Sigsymbols. Wauconda, IL: Don Johnston Developmental Inc.

Green, V. A., Sigafoos, J., Didden, R., O’Reilly, M., Lancioni, G. E., Ollington, N., et al. (2008). Validity of a structured interview protocol for assessing children's preferences. In P. Grotewell \& Y. Burton (Eds.), Early childhood education: Issues and developments (pp. 87-103). New York: Nova Science Publishers.

Gregory, M., DeLeon, I., \& Richman, D. (2009). The influence of matching and moto-imitation abilities on rapid acquisition of manual signs and exchange-based communication responses. Journal of Applied Behavior Analysis, 42, 399-404.

Iacono, T., \& Duncum, J. (1995). Comparison of sign alone and in combination with an electronic communication devices in early language intervention: Case study. Augmentative and Alternative Communication, 11, 249-259.

Jeanes, R., Reynolds, B., \& Coleman, B. (1989). Dictionary of Australasian signs (2nd ed.). Melbourne: Victorian School for Deaf Children.

Kennedy, C. H. (2005). Single-case designs for educational research. Boston: Pearson Education.

Lancioni, G. E., O'Reilly, M. F., Cuvo, A. J., Singh, N. N., Sigafoos, J., \& Didden, R. (2007). PECS and VocAs to enable students with developmental disabilities to make requests: An overview of the literature. Research in Developmental Disabilities, 28, 468-488.

Mayer-Johnson Co. (1994). Picture communication symbols combination book. Solano Beach, CA: Mayer-Johnson Co.

Mirenda, P. (2003). Toward functional augmentative and alternative communication for students with autism: Manual signs, graphic symbols, and voice output communication aids. Language, Speech, E Hearing Services in Schools, 34, 203-216.

National Research Council. (2001). Educating children with autism. Washington, DC: Henry (Joseph) Press.

Pace, G., Ivancic, M., Edwards, G., Iwata, B., \& Page, T. (1985). Assessment of stimulus preference and reinforcer value with profoundly retarded individuals. Journal of Applied Behavior Analysis, 18, 249-255.

Peeters, T., \& Gillberg, C. (1999). Autism: Medical and educational aspects. London: Whurr.

Reichle, J. (1991). Defining the decisions involved in designing and implementing augmentative and alternative communication systems. In J. Reichle, J. York, \& J. Sigafoos (Eds.), Implementing augmentative and alternative communication: Strategies for learners with severe disabilities (pp. 39-60). Baltimore: Paul H.

Rispoli, M., Franco, J. H., van der Meer, L., Lang, R. B., \& Carmargo, S. (2010). The use of speech generating devices in communication interventions for individuals with developmental disabilities: A review of the literature. Developmental Neurorehabilitation, 13, 276-293.

Rotholz, D. A., \& Berkowitz, S. F. (1989). Functionality of two modes of communication in the community by students with developmental disabilities: A comparison of signing and communication books. Journal of the Association for Persons with Severe Handicaps, 14, $227-233$.

Schlosser, R., Blischak, D. M., \& Koul, R. (2003). Roles of speech-output in AAC. In R. Schlosser (Ed.), The efficacy of augmentative and alternative communication: Toward evidence-based practice (pp. 471-532). San Diego: Academic Press.

Schlosser, R., \& Lee, D. L. (2000). Promoting generalization and maintenance in augmentative and alternative communication: A meta-analysis of 20 years of effectiveness research. Augmentative and Alternative Communication, 16, 208-226.

Schlosser, R., \& Blischak, D. M. (2001). Is there a role for speech output in interventions for persons with autism? Focus on Autism E' Other Developmental Disabilities, $16,170$.

Sigafoos, J. (1998). Choice making and personal selection strategies. In J. K. Luiselli \& M. Cameron (Eds.), Antecedent control: Innovative approaches to behavioral support (pp. 187-221). Baltimore: Paul H.

Sigafoos, J. (2006). Self-determination: Can we let the child determine the "best" treatment? Pediatric Rehabilitation, 9, 1-2.

Sigafoos, J., \& Drasgow, E. (2001). Conditional use of aided and unaided AAC: A review and clinical case demonstration. Focus on Autism E Other Developmental Disabilities, 16, 152-161.

Sigafoos, J., Drasgow, E., \& Schlosser, R. (2003). Strategies for beginning communicators. In R. Schlosser (Ed.), The efficacy of augmentative and alternative communication: Toward evidence-based practice (pp. 246-323). San Diego: Academic Press.

Sigafoos, J., Green, V. A., Payne, D., Son, S. H., O’Reilly, M., \& Lancioni, G. E. (2009). A comparison of picture exchange and speech-generating devices: Acquisition, preference, and effects on social interaction. Augmentative and Alternative Communication, 25, 99-109.

Sigafoos, J., \& Iacono, T. (1993). Selecting augmentative and alternative communications devices for persons with severe disabilities: Some factors for educational teams to consider. Australian and New Zealand Journal of Developmental Disabilities, 18, 133-146.

Sigafoos, J., O’Reilly, M., Ganz, J. B., Lancioni, G. E., \& Schlosser, R. W. (2005). Supporting self-determination in AAC interventions by assessing preference for communication devices. Technology and Disability, 17, 1-11.

Son, S. H., Sigafoos, J., O’Reilly, M., \& Lancioni, G. E. (2006). Comparing two types of augmentative and alternative communication systems for children with autism. Pediatric Rehabilitation, 9, 389-395.

Soto, G., Belfiore, P. J., Schlosser, R., \& Haynes, C. (1993). Teaching specific requests: A comparative analysis of skill acquisition and preference using two augmentative and alternative communication aids. Education and Training in Mental Retardation, 28, $169-178$.

Stafford, A., Alberto, P., Fredrick, L., Heflin, L., \& Heller, K. (2002). Preference variability and the instruction of choice making with students with severe intellectual disabilities. Education and Training in Mental Retardation and Developmental Disabilities, 37, 70-88.

Tincani, M. (2004). Comparing the picture exchange communication system and sign language training for children with autism. Focus on Autism $\mathcal{E}$ Other Developmental Disabilities, $19,152-163$.

van der Meer, L., \& Rispoli, M. (2010). Communication interventions involving speech-generating devices for children with autism: A review of the literature. Developmental Neurorehabilitation, 13, 294-306.

Weitz, C., Dexter, M., \& Moore, J. (1997). AAC and children with developmental disabilities. In S. Glennen \& D. DeCoste (Eds.), Handbook of augmentative and alternative communication (pp. 395-431). San Diego, CA: Singular.

Winborn-Kemmerer, L., Ringdahl, J. E., Wacker, D. P., \& Kitsukawa, K. (2009). A demonstration of individual preference for novel mands during functional communication training. Journal of Applied Behavior Analysis, 42, 185-189.

Wing, L., \& Attwood, A. (1987). Syndromes of autism and atypical development. In D. J. Cohen \& A. M. Donnellan (Eds.), Handbook of autism and pervasive developmental disorders (pp. 3-19). New York: Wiley. 


\section{APPENDIX E}

van der Meer, L., Kagohara, D., Achmadi, D., O'Reilly, M., Lancioni, G., Sutherland, D., \& Sigafoos, J. (2012). Speech-generating devices versus manual signing for children with developmental disabilities. Research in Developmental Disabilities, 33, 1658-1669 
Provided for non-commercial research and education use. Not for reproduction, distribution or commercial use.

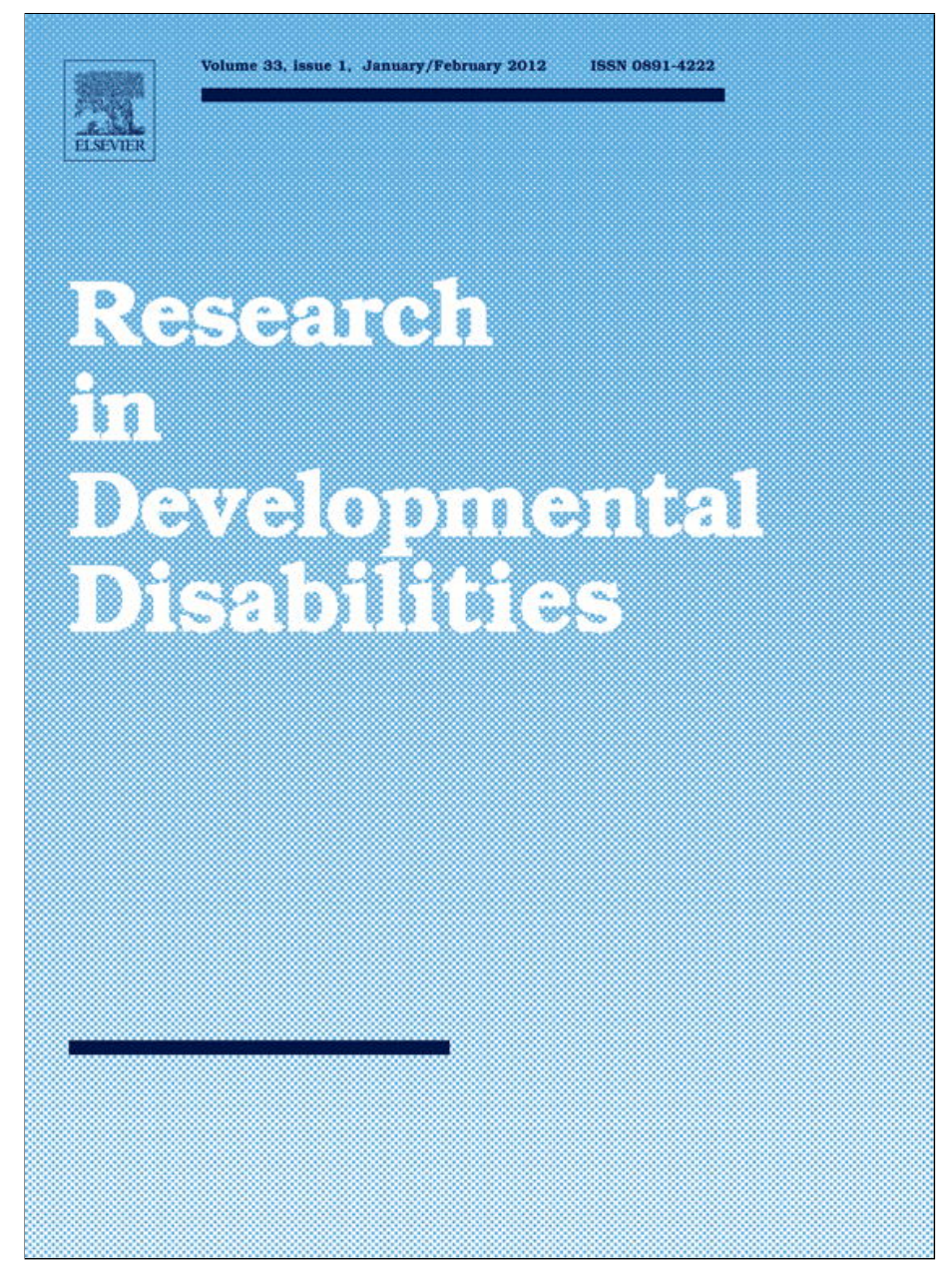

(This is a sample cover image for this issue. The actual cover is not yet available at this time.)

This article appeared in a journal published by Elsevier. The attached copy is furnished to the author for internal non-commercial research and education use, including for instruction at the authors institution and sharing with colleagues.

Other uses, including reproduction and distribution, or selling or licensing copies, or posting to personal, institutional or third party websites are prohibited.

In most cases authors are permitted to post their version of the article (e.g. in Word or Tex form) to their personal website or institutional repository. Authors requiring further information regarding Elsevier's archiving and manuscript policies are encouraged to visit:

http://www.elsevier.com/copyright 


\title{
Speech-generating devices versus manual signing for children with developmental disabilities
}

\author{
Larah van der Meer $^{\mathrm{a}, *}$, Debora Kagohara ${ }^{\mathrm{a}}$, Donna Achmadi ${ }^{\mathrm{a}}$, Mark F. O’Reilly ${ }^{\mathrm{b}}$, \\ Giulio E. Lancioni ${ }^{\mathrm{c}}$, Dean Sutherland ${ }^{\mathrm{d}}$, Jeff Sigafoos ${ }^{\mathrm{a}}$ \\ ${ }^{a}$ Victoria University of Wellington, New Zealand \\ ${ }^{\mathrm{b}}$ Meadows Center for the Prevention of Educational Risk, University of Texas at Austin, Texas, USA \\ ${ }^{\mathrm{c}}$ University of Bari, Italy \\ ${ }^{\mathrm{d}}$ University of Canterbury, Christchurch, New Zealand
}

\section{A R T I C L E I N F O}

\section{Article history:}

Received 3 April 2012

Accepted 5 April 2012

Available online

\section{Keywords:}

Augmentative and alternative

communication

Communication intervention

Developmental disability

Manual signing

Preference assessment

Speech-generating device

\begin{abstract}
A B S T R A C T
We compared speed of acquisition and preference for using a speech-generating device (SGD) versus manual signing (MS) as augmentative and alternative communication (AAC) options. Four children with developmental disabilities (DD), aged 5-10 years, were taught to request preferred objects using an $\mathrm{iPod}^{\mathbb{R}}$-based SGD and MS. Intervention was introduced in a multiple-probe across participants design and SGD and MS conditions were compared in an alternating treatments design. A systematic choice-making paradigm was implemented to determine if the children showed a preference for using SGD or MS. All participants showed increased use of SGD when intervention was introduced, but only three learned under the MS condition. Three participants exhibited a preference for the SGD while the remaining participant demonstrated a preference for using MS. Results support previous studies showing that individuals with DD often show a preference for different AAC options and extend previous data by suggesting that acquisition and maintenance was better for the preferred option.
\end{abstract}

(c) 2012 Elsevier Ltd. All rights reserved.

\section{Introduction}

Children with developmental disabilities (DD) often fail to develop the ability to speak (National Research Council, 2001; Peeters \& Gillberg, 1999) and therefore need intervention to establish augmentative and alternative communication (AAC; Beukelman \& Mirenda, 2005; Schlosser, 2003a). Various AAC options have been developed, including unaided approaches, such as gestures and manual signing (MS); and aided systems, which involve the use of external equipment to enable the individual to communicate. Examples of aided AAC include low-tech communication boards (Sigafoos \& Iacono, 1993), the Picture Exchange Communication System (PECS; Bondy \& Frost, 2001), and speech-generating devices (SGDs; Lancioni et al., 2007).

Two recent reviews, summarizing 58 studies, provide empirical support for the use of SGDs in communication interventions for individuals with DD (Rispoli, Franco, van der Meer, Lang, \& Carmargo, 2010; van der Meer \& Rispoli, 2010). Results from these reviews support a conclusion that individuals with DD can learn to use a range of SGDs for functional communication. Similarly, several systematic reviews of the literature (e.g., Goldstein, 2002; Schlosser \& Wendt, 2008a,b;

\footnotetext{
* Corresponding author at: School of Educational Psychology and Pedagogy, Victoria University of Wellington, PO Box 17-310, Karori 6147, Wellington, New Zealand. Tel.: +644 $4635233 \times 4597$.

E-mail address: larah.vandermeer@vuw.ac.nz (L. van der Meer).
} 
Wendt, 2009) pertaining to the use of MS provide results from more than 20 studies demonstrating that MS use can also be taught to individuals with DD.

Despite this supporting evidence, there is considerable debate within the literature regarding which of the various aided and unaided AAC options is best suited to individuals with DD (Mirenda, 2003; Schlosser \& Blischak, 2001). There does not appear to be one AAC system that is appropriate for all beginning communicators (Sigafoos, Drasgow, \& Schlosser, 2003). For example, studies comparing the acquisition of different AAC options have revealed few major or consistent differences in terms of acquisition for PECS and MS (Adkins \& Axelrod, 2001; Gregory, DeLeon, \& Richman, 2009; Rotholz, Berkowitz, \& Burberry, 1989; Tincani, 2004), as well as PECS and SGDs (Beck, Stoner, Bock, \& Parton, 2008; Bock, Stoner, Beck, Hanley, \& Prochnow, 2005). However, few studies have compared SGDs and MS (Iacono \& Duncum, 1995; Iacono, Mirenda, \& Beukelman, 1993; Sigafoos \& Drasgow, 2001).

The lack of any major difference in these studies suggests that how quickly an individual acquires an AAC option may not be the most critical variable to examine when considering which AAC option to implement. Because individuals with DD are a heterogeneous group, general statements about successful or unsuccessful interventions could be seen as providing relatively less guidance when selecting an AAC approach for a specific person. Selecting a suitable AAC system for an individual can therefore be a difficult process that is more likely to depend on specific task demands and individual characteristics (Sigafoos et al., 2003; Ringdahl et al., 2009; Wendt, 2009). For example, an individual's preference for using one AAC option over another may be an important variable to consider when selecting an appropriate AAC system. Giving students the opportunity to self-select their most preferred AAC option could also be viewed as one way of promoting selfdetermination in AAC interventions (Sigafoos, 2006), and might significantly influence progress in learning to communicate.

Indeed, several studies have assessed personal preferences between AAC devices in communication interventions for individuals with DD (Cannella-Malone, DeBar, \& Sigafoos, 2009; Sigafoos, Green, et al., 2009; Sigafoos, O’Reilly, Ganz, Lancioni, \& Schlosser, 2005; Son, Sigafoos, O’Reilly, \& Lancioni, 2006; Soto, Belfiore, Schlosser, \& Haynes, 1993; WinbornKemmerer, Ringdahl, Wacker, \& Kitsukawa, 2009). These studies implemented a structured choice-making arrangement (e.g., Sigafoos, 1998) to identify a participant's preference for one mode of communication over another. The general approach used in these studies was to teach individuals to use two different AAC options for functional communication (e.g., requesting to play with a toy). The next step was to give the individual an opportunity to choose which AAC option (SGD versus PECS) to use. When one option was consistently chosen over another, it was considered to be the participant's preferred mode of communication.

Evidence from a systematic review of the literature (van der Meer, Sigafoos, O’Reilly, \& Lancioni, 2011) pertaining to these studies indicated that, although individuals with DD did often demonstrate a preference for one AAC device over another, these studies were limited in a number of ways. Specifically, the choice-making preference assessments were post hoc in that they were undertaken only after learning to use each communication option. Also, only one of these studies (Soto et al., 1993) assessed the influence of preference on maintenance of communication skills. It therefore remains unclear whether preference can be identified during and incorporated into the beginning stages of AAC intervention and whether utilizing a learner's preferred AAC option might actually improve intervention outcomes in terms of maintenance of newly acquired communication skills.

In six of the seven studies synthesized by van der Meer, Sigafoos, et al. (2011), just two AAC options (PECS and SGD) were compared. Only one study (Iacono \& Duncum, 1995) compared the use of MS and a SGD. In this latter study, however, instead of implementing a choice-making arrangement, the most effective treatment modality was considered to be participant's preferred AAC option. This may be due to some of the apparent difficulties in assessing preferences between aided and unaided AAC. Sigafoos et al. (2005) explained that it is probably more complex to assess preference for using MS versus a SGD, for example, because it would seem more difficult to present the MS option as concretely as one can present a SGD or a PECS board. They therefore suggested teaching the use of MS in the presence of one distinctive stimulus and the use of SGD in the presence of another distinctive stimulus. A choice condition could then be arranged by presenting both of these distinctive stimuli.

The present study aimed to employ such a procedure in order to assess preference for aided (SGD) versus unaided (MS) modes of AAC. Previous research highlighted the importance of utilizing more sophisticated AAC devices that allow for individuals to expand on their communication capabilities (Cannella-Malone et al., 2009), yet remain cost effective (Sigafoos et al., 2005). Therefore the present study used a new, empirically validated (Kagohara et al., 2010; van der Meer, Kagohara, et al., 2011), software application known as Proloquo2Go ${ }^{\mathrm{TM}}$ (Sennott \& Bowker, 2009) run on an iPod ${ }^{\mathbb{R}}$-based SGD, as well as signs from the Makaton (Makaton New Zealand/Aotearoa, 1998-1999) sign language vocabulary. In comparing preferences for this iPod ${ }^{\circledR}$-based SGD versus MS, the current study also aimed to address some of the aforementioned limitations of AAC device preference studies. Specifically, we aimed to determine whether the participants made greater progress, showed increased communication ability, and showed better maintenance of the AAC system for which they showed a preference. It was hypothesized that acquisition and maintenance of the two AAC options would be better for the AAC option that the participants also showed a preference for using.

\section{Method}

\subsection{Participants}

Four children were recruited from a special education unit in a public school. All four participants met the following criteria: (a) diagnosis of intellectual/developmental disability or ASD, (b) school aged children of less than 18 years of age, 
(c) very limited or no communication skills determined by an age level of 2.5 years or less in the Expressive Communication Domain of the Vineland Adaptive Behavior Scales (Vineland-II; Sparrow, Cicchetti, \& Balla, 2005), (d) no auditory or visual impairments that would interfere with the use of AAC, and (e) sufficient motor skills to operate the AAC communication system, determined by an age level of 1.0 year or more on the Fine Motor Skills Domain of the Vineland-II.

David was a 10-year-old male diagnosed with ASD. On the communication sub-domain of the Vineland-II (Sparrow et al., 2005), David received age equivalencies of 3:11 (years:months) for receptive communication, 1:1 for expressive communication, and 4:3 for written communication. David did not have any spoken language. At the time of the study he was using the Informative Pointing Method (Iversen, 2007), with assistance, as a form of communication. David had limited experience with Makaton Keyword Signing and PECS for expressive and receptive language prior to the current intervention. In addition to his severe communication impairment, David had limited social skills and engaged in frequent problem behavior, including self-injurious behavior.

Tom was a 5.5-year-old boy diagnosed with Multi-System Developmental Disorder (MSDD) with autistic-like behaviors (e.g., impairment in reciprocal social communication, stereotyped and repetitive behaviors). His age equivalencies on the communication domain of the Vineland-II (Sparrow et al., 2005) were 0:11, 1:0, and 2:5 for receptive, expressive, and written communication. Tom was able to use a few single words to make requests and comment on his environment, but he rarely spoke unless he was prompted by an adult. Prior to the current intervention Tom had been introduced to Makaton Keyword Signing and PECS for receptive and expressive language, with limited success.

Zac was a 7-year-old male diagnosed with Down syndrome and ASD. He received age equivalencies of 1:3, 2:1, and 4:10 on the receptive, expressive, and written communication domains of the Vineland-II (Sparrow et al., 2005). Prior to intervention Zac used Makaton Keyword Signing and PECS for expressive and receptive language. During the current intervention Zac received a GoTalk 2.0 ${ }^{\circledR}$ SGD, which his teachers started to use with him. Although Zac attempted to speak frequently, his speech was largely unintelligible.

Eli was a 5.5-year-old male with Congenital Myotonic Dystrophy and autistic-like behaviors (e.g., impairment in reciprocal social communication, stereotyped and repetitive behaviors). His age equivalencies were 0:11, 0:8, and 2:5 on the receptive, expressive, and written communication domains of the Vineland-II (Sparrow et al., 2005). Eli did not have any spoken language. He was introduced to Makaton Keyword Signing for receptive and expressive language prior to the current intervention. He could use the signs for MORE and EAT. Aside from this, Eli made few communicative attempts. He engaged in frequent stereotypic (self-stimulatory) behavior and was said to often fixate on certain objects, such as computers.

\subsection{Setting and intervention context}

All four participants attended the same classroom at a special education unit for children with disabilities that was part of a public primary school. Fourteen additional children were educated in this classroom by two teachers and six teaching assistants. The procedures related to this study were conducted at a table in a small room that linked to the main classroom during a morning snack/leisure activity. The procedures were implemented in a one-to-one context consisting of the trainer (trained graduate assistant) and one participant at a time.

\subsection{Preferred stimuli}

Snacks and/or toys that the participants seemed to prefer, and which would be appropriate for the participants to request during the snack/leisure activity, were identified by a systematic two-stage stimulus assessment (Green et al., 2008). Stage 1 involved an indirect assessment in which both teachers were asked to list snack foods, sensory stimuli, and toys that the participants appeared to enjoy and would be appropriate for the classroom. Three to six of the most preferred food and play stimuli were then selected for a direct stimulus assessment. The direct method for assessing preferences involved the simultaneous presentation of multiple items, without replacement (DeLeon \& Iwata, 1996; Duker, Didden, \& Sigafoos, 2004). Each participant was presented with an array of preferred items (random placement) and allowed to select one. Items were not replaced once they had been selected, thereby eliminating the chance of the participant choosing only one or a few items, as well as allowing the trainer to develop a rank order of items in terms of preference. Play and food items were assessed separately. The top two to four food and play items were identified by calculating the percentage of times that the stimulus was selected, out of six trials (across four sessions) in which each stimuli were offered.

Preferred stimuli for David included a venting ball (46\%), dental floss (43\%), and water play (represented by a container filled with water and plastic toys; $29 \%$ ) for toy items; fruit-leather (66\%), M\&Ms (40\%), and chocolate and gherkins (33\% each) for snack items. Preferred stimuli for Tom included toy cars (100\%), bubbles (42\%), and building blocks (35\%) and a lollipop (86\%), muesli bar (42\%), and crackers (22\%). Preferred stimuli for Zac included an alphabet musical toy (55\%), a Hi5 CD (40\%), and playing on the computer (30\%) and juice (50\%) and chips (20\%). Preferred items for Eli were bubbles (67\%), paper (43\%), and an alphabet musical toy (38\%) and peppermints (75\%), chips (46\%), and juice (31\%). All participants, excluding Zac (his parents indicated a preference for teaching play items, not snacks), received baseline with both the preferred snack and play options. David initially received training to request both snack and play items. However, due to time constraints it was decided that David would be taught to request snacks only (at Session 8 of Fig. 1), while Tom, Zac, and Eli were taught to request toys only. Snacks for David and toys for the other children were identified as the more preferred options in the direct stimulus assessment. 


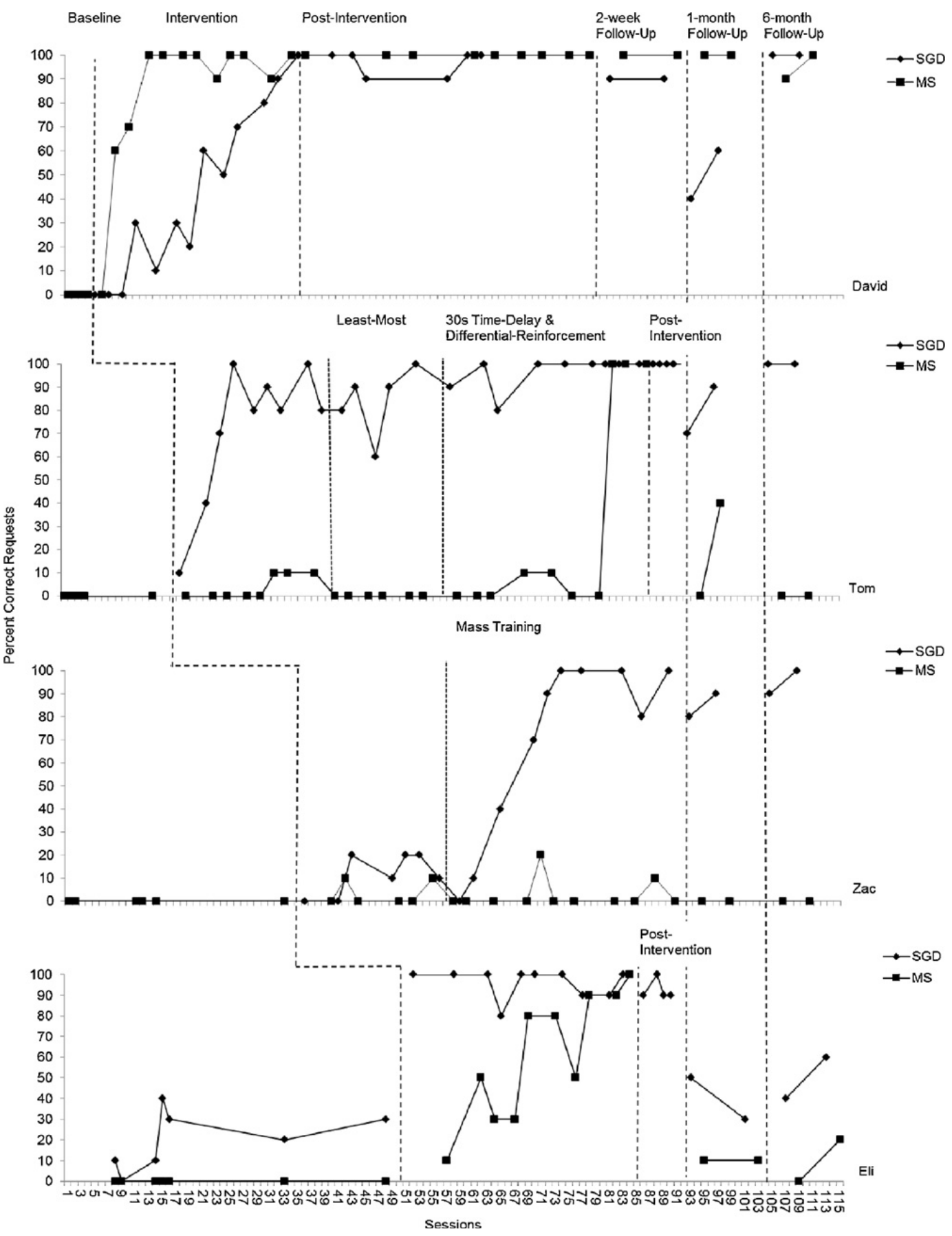

Fig. 1. Percentage of correct requests using the SGD and MS options across sessions for each participant.

\subsection{Speech-generating device}

Participants were taught to request their preferred toys or snacks using an Apple iPod Touch ${ }^{\circledR}$ with Proloquo2Go ${ }^{\mathrm{TM}}$ software. The $\operatorname{iPod}^{\circledR}$ was placed inside an iMainGo $2^{\circledR}$ speaker case to increase sound amplification. The iPod ${ }^{\circledR}$ was configured to show a single page containing three $(2.5 \mathrm{~cm} \times 2.5 \mathrm{~cm})$ graphic symbols, representing requests for $S N A C K S$, PLAY, and SOCIAL INTERACTION. Touching each symbol activated corresponding synthetic speech-output (i.e., "I want a snack please.", "I want to play.", and "How are you?").

\subsection{Manual signing}

Participants were also taught to request preferred stimuli using manual signs from the Makaton Sign Language system (Makaton New Zealand/Aotearoa, 1998-1999). Participants were taught the signs for SNACK or PLAY. The MS option was represented by a laminated photo $(16 \mathrm{~cm} \times 11 \mathrm{~cm})$ of the trainer making the hand formations for the signs for SNACK and PLAY. 


\subsection{Response definitions and measurement}

For SGD use, correct responding was defined as independently (without a gestural or verbal prompt) touching the symbol on the screen of the SGD to activate the corresponding speech output in exchange for a desired item from the trainer. MS was defined as independent (without a gestural or verbal prompt) hand gestures to produce correct signs in exchange for a desired item from the trainer. The frequency of correct responding was calculated for each session consisting of 10 offers of snack or play items. The target response for David was touching the SNACK symbol on the SGD to activate the message "I want a snack please.". The PLAY and SOCIAL INTERACTION symbols were distracters. David's target response for MS was to produce the sign for SNACK. The PLAY sign (on the laminated card) was a distractor. The target response for Tom, Zac, and Eli was touching the PLAY symbol on the SGD to activate the message "I want to play.". The SNACK and SOCIAL INTERACTION symbols were distracters. The MS target response for Tom, Zac, and Eli was to produce the sign for PLAY. The SNACK sign (on the laminated card) was a distractor.

\subsection{Experimental design}

The study included the following phases arranged in a multiple-probe across participants design (Kennedy, 2005): Baseline, Intervention, Preference Assessment (throughout Intervention, Post-Intervention, and Follow-Up), PostIntervention, and Follow-Up. An alternating-treatments design was also embedded within the multiple-probe to compare performance of the SGD and MS options.

\subsection{Session schedule}

Two to four requesting sessions were conducted three to four days per week. Each session lasted about 5 min and consisted of 10 discrete trials. The AAC option available during a session (i.e., SGD versus MS) was alternated across sessions. Whether training commenced with SGD or MS was random depending on how many sessions were implemented on the previous day of training. For all sessions, the participant and trainer were seated next to each other at a table with one or two additional reliability and procedural integrity observers seated nearby.

\subsection{Procedures}

Because participants were at the beginning stages of intervention they were taught to request only one type of highly preferred item. However, to ensure some level of symbol discrimination, the distractor symbols/signs were included in the SGD and MS options. If participants requested a snack when they were undertaking training to request a toy or vice versa, the trainer explained We are learning to request toys/snack at the moment, you can request a toy/snack another time. Activating the SOCIAL INTERACTION symbol on the SGD resulted in a brief spoken reply from the trainer (e.g., I'm good thanks, how about you?). It was considered more natural to provide participants with some feedback when they activated these (non-target) symbols/produced these (non-target) signs, but not to reinforce these with preferred tangibles. Similarly, producing MS to request items during SGD sessions was ignored in order to bring the use of each option under stimulus control.

\subsubsection{Baseline}

During baseline, a tray containing three different snack (toy) items was placed on the table in view, but out of the participants' reach. The SGD was placed on one side of the table in an upright orientation. A photo of the trainer signing was used to present the MS option. The photo was placed on the other side of the table, also in an upright orientation. Each session involved one block of five discrete trials for snacks and one block of five discrete trials for toys, with the order of blocks counterbalanced across sessions. The session began with the trainer telling the participant: Here is a tray of snacks (toys), let me know if you want something. After $10 \mathrm{~s}$, the trainer moved the tray within reach and allowed the participant to take one item. This was repeated across 5 discrete trials each for snacks and toys. This 10 -s fixed time schedule of reinforcement was provided to ensure continued motivation to participate in sessions. When offering snacks, participants were allowed to select one item from the tray, which was then replenished before the next offer. When offering the tray of toys, participants were allowed to select one toy and play with it for approximately $30 \mathrm{~s}$ before it was returned to the tray. SGD and MS responses were recorded, but had no programmed consequences.

\subsubsection{Intervention}

This phase was conducted in a discrete trial format until participants reached criterion (i.e., $80 \%$ correct requesting across 3 consecutive sessions for each AAC option). Either the SGD or the MS option was placed on the table (see Session Schedule) in reach of the child in accordance with the alternating treatments design. Each trial consisted of the trainer pointing to a tray of toys (snacks) and saying: Here's a tray of toys (snacks). Let me know if you want something. Training involved a 10-s time delay between the verbal cue (i.e., Let me know if you want something) and the use of graduated guidance to prompt a correct request. Graduated guidance involved use of the least amount of physical guidance necessary to ensure the child made a correct request, while simultaneously explaining the required response (e.g., Press PLAY to ask to play with a toy. or Move your 
hand to your mouth to make the sign for SNACK.). Immediately after the speech output occurred, or the correct sign was made, the trainer moved the tray containing the toys (or snacks) within reach of the participant. The participant was allowed to select one item from the tray and consume the chosen snack or play with the chosen toy for about $30 \mathrm{~s}$. After this, the next trial was initiated.

\subsubsection{Procedural modifications}

Because Tom made little progress with acquisition of MS during the initial intervention sessions, his teaching procedures were modified to include 10-s time delay followed by a least-to-most prompting procedure. This involved first tapping Tom's elbow, then lifting his forearm, followed by a full physical prompt if he did not make the sign correctly. However, because progress was not observed with this procedure, a 30-s time delay followed by graduated guidance, as well as differential reinforcement (where Tom was only given the opportunity to play if he independently used the sign to request to play), was introduced. Prompted trials were not reinforced.

Another participant $(\mathrm{Zac})$ did initiate correct requests during the initial intervention sessions, but he appeared to have trouble physically performing the task independently for both MS and the SGD. It was therefore decided to implement massed training trials before each SGD and each MS session. This involved 10 consecutive graduated guidance trials with no time delay and no reinforcement per session to get Zac to perform the task as independently as possible.

\subsubsection{AAC preference assessments}

These assessments were undertaken to determine if participants would show a preference for using one of the two AAC options. These assessments occurred after every eighth session (i.e., after four MS and four SGD sessions) on average. This number varied slightly because these assessments had to occur before the first session for the day to prevent sequence effects (selecting the AAC option that was taught last; Sigafoos et al., 2005), as well as to ensure that at least two such preference assessments were undertaken during intervention for each participant.

During a preference assessment, the trainer presented the MS option on one side of the table and the SGD option on the other side of the table (alternated across sessions to control for choice being made dependent on location of the AAC option). The trainer asked the participant: Which communication option would you like to use? Sign language on this side (while pointing), or the SGD on this side (while pointing)? The trainer initiated one requesting opportunity with the chosen AAC option before reverting back to initiating requesting opportunities with the AAC device that was being used for that session. Choice for an AAC option was defined as physically pointing to, touching, or picking up the selected communication option. If the child did not choose an option within $10 \mathrm{~s}$, the device preference assessment was terminated and training continued with the AAC option that was scheduled for use in that session.

\subsubsection{Post-intervention}

Once the participant reached criterion with both the MS and SGD options, post-intervention preference assessments were introduced. These were identical to previously described device preference assessments, except that once an AAC option had been chosen, the participant continued to request preferred items using the chosen communication method for the entire session. A minimum of four of these post-intervention preference assessments were undertaken for each participant.

\subsubsection{Follow-up}

David received three sets of follow-up sessions, at 2 weeks, 1 month, and 6 months. The remaining participants received two sets of follow-up sessions at 1 month and 6 months. David received four follow-up sessions two weeks after his last post-intervention session. During the two-week interval, David was on midwinter break, did not attend school, and did not use either communication option. All participants received four follow-up sessions one month after their final intervention or post-intervention session. Participants did not use either communication option for requesting preferred items during the one month interval before these follow-up sessions. Six months later, another four follow-up sessions were conducted for all participants. During the interval between the 1 and 6 month follow-up sessions, the participants had access to iPods ${ }^{\mathbb{R}}$ and iPads ${ }^{\mathbb{R}}$ for educational and leisure activities, but did not use the iPod ${ }^{\mathbb{R}}$-based SGD or MS communication options for requesting preferred items. Procedures for follow-up were identical to the intervention phase, except no teaching occurred and reinforcement was contingent upon a correct request. A device preference assessment was implemented before each session.

\subsection{Inter-observer agreement}

The trainer collected data on the frequency of correct requesting, the level of prompting required during intervention for each trial, as well as which communication mode was selected during device preference assessments. To assess the reliability of the trainer's data collection, an independent observer also collected data on the frequency of requesting, level of prompting, and communication mode chosen. For each session, percentages of agreement between the independent observer and the trainer were calculated using the formula:agreements/(agreements + disagreements) $\times 100$. Reliability of data collection was obtained by the independent observer on $43 \%$ of all sessions and ranged from $90 \%$ to $100 \%$ with an overall mean of $99.6 \%$. 


\subsection{Procedural integrity}

To assess procedural integrity, the independent observer used a checklist of the procedures and recorded whether or not the trainer had correctly implemented each procedural step in its proper sequence. The independent observer assessed procedural integrity during $43 \%$ of all sessions, which ranged from $98 \%$ to $100 \%$ with an overall mean of $99.9 \%$. A second independent observer collected inter-observer agreement data on $21 \%$ of these integrity checks with $100 \%$ agreement.

\section{Results}

Fig. 1 shows the percentage of correct requests during each session for both AAC modes across participants. Fig. 2 provides a summary of the results from the AAC preference assessments across each phase of the study. During baseline, participants never used MS to request snacks or toys. They did occasionally touch the MS card, pick it up, or play with it for a few seconds. Eli used the SGD to request toys or snacks on $20 \%$ of the baseline trials. It should be noted that many of these requests appeared to be made by chance as Eli repeatedly touched the icons on the SGD representing items (snack or toy) that were not being offered on that trial. Although none of the other participants activated the SGD, they did occasionally touch it, pick it up, or play with it for a few seconds. Also, during baseline, all participants often reached for the tray of snacks or toys before it was offered to them (these responses were blocked). Zac attempted to verbally request certain items from the tray, but this speech was mostly unintelligible. When the tray of snacks or toys was offered, David and Tom selected and consumed or played with items $98 \%$ of the time, Zac did so $100 \%$ of the time, and Eli did so $94 \%$ of the time.

\subsection{David}

David achieved acquisition of requests for snacks using MS on his 6th MS session (Session 18 of Fig. 1). He achieved acquisition using the SGD on his 13th SGD session (Session 35 of Fig. 1). During intervention, David received four device preference assessments in which he chose MS on each occasion. A second device preference assessment was undertaken for David three sessions after the first to ensure the accuracy of the initial device preference assessment because a correct response had to be prompted. Because the results were consistent, this was not repeated for other participants where a response had to be prompted. During the post-intervention phase David chose MS on $60 \%$ of sessions and used it with $100 \%$ proficiency during subsequent requesting opportunities. He chose the SGD 40\% of the time and correctly used it 90-100\% of the time.

During his first set of follow-up sessions, David maintained requesting for snacks using both the SGD and MS at a high level. He chose the MS option on three out of four (75\%) preference assessment opportunities and used it with $100 \%$ proficiency. While MS maintained at $100 \%$ correct requesting during his second set of follow-up sessions (one month after

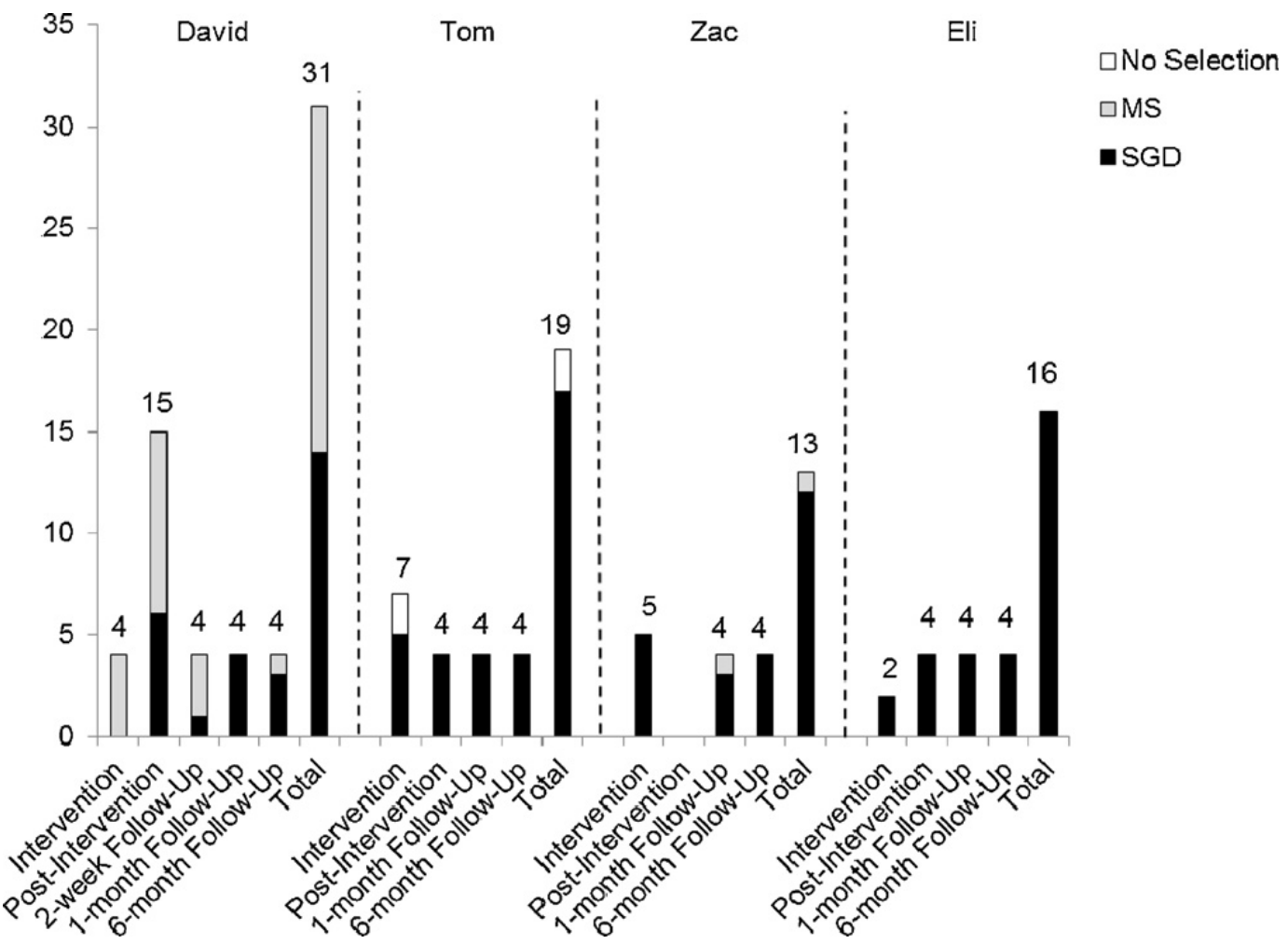

Fig. 2. Results from the device preference assessment probes depicting the number of times each communication option (SGD and MS) was chosen and number of times a device was not chosen (no selection) across each phase of the study for each participant. 
the initial follow-up), SGD use dropped to $40-60 \%$ correct requesting. Interestingly, David chose the SGD on $100 \%$ of the device preference assessments, but on three out of these four occasions he did not make a correct request using the SGD. At the six month follow-up, MS use maintained at 90-100\% correct and David demonstrated increased SGD performance with $100 \%$ correct requesting. Again, David showed a preference for the SGD, selecting it on 75\% (3 out of 4) of opportunities. Overall David received a total of 31 opportunities to choose between the SGD and MS (Fig. 2) and chose MS (55\%) on a slightly higher percentage of occasions.

\subsection{Tom}

Tom achieved acquisition of requests for toys using the SGD on his 6th SGD session (Session 31 of Fig. 1), but he made little progress with MS. On his 9th MS training session (Session 41 of Fig. 1), the least-to-most prompting procedure with a 10-s time delay was implemented during MS sessions. Across all MS sessions, Tom needed all three levels of prompting. By his 15th MS session (Session 59 of Fig. 1), little progress was observed and it appeared that Tom was becoming prompt dependent. Thus the 30-s time delay followed by graduated guidance, as well as differential reinforcement was introduced. Tom had shown slight improvement during initial graduated guidance sessions, but no improvement with least-most prompting; it was therefore thought that reverting back to graduated guidance with a greater time delay and differential reinforcement might increase opportunities for independent responding. With this new prompting procedure, Tom demonstrated $100 \%$ correct requesting at his 22nd MS session and achieved the acquisition criterion by his 24 th signing session (Session 87 of Fig. 1). Anecdotally, throughout both SGD and MS sessions Tom demonstrated an increase in his spoken language. He often imitated the trainer's words surrounding the play activity and even spontaneously spoke several words and short phrases.

Throughout intervention Tom received seven device preference assessments. He consistently chose the SGD and used it correctly to make a request. On two occasions, however, Tom did not make a choice for MS or SGD. During post-intervention preference assessments Tom received a total of four choice opportunities. He chose the SGD on each occasion and achieved $100 \%$ correct requesting with it. Tom maintained a high level of correct requesting using the SGD during the one month follow-up and chose to use it on each device preference assessment. His MS use ranged from $0 \%$ to $40 \%$ correct. At the six month follow-up, Tom's preference for, and correct use of, the SGD (both 100\%) maintained. Correct use of MS dropped completely to 0\%. Overall Tom received 19 preference assessments (Fig. 2) and chose the SGD the majority of the time (89\%).

\subsection{Zac}

Zac made little progress to request with either AAC option. With the implementation of mass training trials (Session 57 of Fig. 1), Zac achieved acquisition using the SGD on his 14th SGD session (Session 77 of Fig. 1). He failed to reach acquisition during MS sessions. Throughout intervention Zac received five device preference assessments. He chose the SGD on each of these assessments. Once he was able to use the SGD independently, he was also able to request to play correctly on these preference assessments. Zac did not progress to the post-intervention phase due to his failure to acquire use of the MS option.

Zac maintained a high level (80-90\%) of correct requesting with the SGD, but he did not make any correct requests with MS during the one month follow-up. Despite not being able to make a correct request with it, Zac did choose the MS option on one preference assessment opportunity, but chose the SGD on the other three assessments. Zac maintained higher levels of correct requesting (90-100\%) and preference (100\%) for SGD during the six month follow-up. His MS remained at 0\% correct. Overall, Zac received 13 AAC preference assessments (Fig. 2) during which he chose the SGD 92\% of the time.

\subsection{Eli}

Eli achieved acquisition of requests for toys using the SGD on his 3rd SGD session (Session 63 of Fig. 1). However, throughout the SGD intervention sessions he made many errors, repeatedly pressing all three icons (SNACK, PLAY, and SOCIAL INTERACTION) on the SGD. Eli achieved acquisition for requesting toys using MS on his 10th MS session (Session 84 of Fig. 1). During the intervention phase he received two device preference assessments each time choosing the SGD and using it correctly to request to play with toys. During the post-intervention preference assessments Eli received a total of four choice opportunities and chose the SGD all four times in which he requested at $90-100 \%$ proficiency.

Eli's performance dropped for both the SGD (30-50\% correct requesting) and MS (10\% correct requesting) during the one month follow-up. He chose the SGD on $100 \%$ of choice opportunities using it with $100 \%$ proficiency on three out of four device preference assessments. As with the other participants, Eli's performance increased during the six month follow-up, with $40-60 \%$ correct requesting with the SGD and 0-20\% correct requesting with MS. Although he chose the SGD on 100\% of opportunities, Eli used it correctly on only one of the four occasions. Overall Eli received 16 opportunities to choose between the SGD and MS option (Fig. 2) during which he always chose the SGD (100\%).

\section{Discussion}

All four participants learned to make augmented requests and demonstrated a preference for using the SGD or MS. Specifically, three (David, Tom, and Eli) of the four boys reached criterion on use of both communication options to request 
preferred stimuli. The fourth boy (Zac) reached criterion for SGD use, but did not learn to use MS. Tom, Zac, and Eli demonstrated a preference for using the SGD, while David exhibited a slight preference for using MS. These results support findings from previous research suggesting that students with DD can learn to use various AAC systems for functional communication and will often demonstrate a preference for one communication device over another (van der Meer, Sigafoos, et al., 2011). Moreover, most of the children assessed to date in this and the previous studies reviewed by van der Meer, Sigafoos, et al. showed a preference for using SGDs over PECS (or MS), although it is important to note that studies comparing SGDs and MS are rare. In fact, the present study appears to be the only study to date that has directly compared acquisition of, and preference for, SGDs versus MS.

However, while results from this systematic review of the preference literature (van der Meer, Sigafoos, et al., 2011) and results from other studies comparing acquisition of various modes of AAC (e.g., Bock et al., 2005; Gregory et al., 2009) highlight few differences in terms of how quickly children learn to use each communication system, outcomes from the current investigation indicate differences in terms of how rapidly participants learned to use each AAC option. Specifically, three of the four participants learned to use the SGD (also their preferred communication option) more quickly than MS. A possible explanation for this difference in findings is that the majority of the previous research did not include a comparison between SGD and MS (e.g., Bock et al., 2005; Gregory et al., 2009). Of the few studies (Iacono \& Duncum, 1995; Iacono et al., 1993; Sigafoos \& Drasgow, 2001) that did compare SGD with MS, the results also showed better performance with the SGD option. Similarly, results from research comparing acquisition of PECS and MS indicate that PECS was learned at a faster rate than MS (Adkins \& Axelrod, 2001; Rotholz et al., 1989; Tincani, 2004), but it is argueable whether these differences were clinically significant. In this and previous studies, SGD use could have been acquired faster because it might be more difficult to teach MS use, rather than it being inherently more difficult for children with DD to learn.

The findings of the present study, when considered in light of previously reviewed studies (van der Meer, Sigafoos, et al., 2011), suggest that there may not be one single mode of AAC that is best for children with DD. Thus decisions as to which AAC option to implement might be based upon an assessment of the individual user's unique abilities, needs, and their preferences for different AAC options (Sigafoos \& Drasgow, 2001). A key aspect of the current study was the assessment of each child's preference for the two AAC options using a structured choice-making protocol (e.g., Sigafoos, 1998). In previous preference research (Sigafoos et al., 2005; Son et al., 2006; Soto et al., 1993), it has been argued that because participants had equal exposure to and achieved equal proficiency with each communication system prior to implementing the choice assessment, they were able to make an informed choice, and that preference for one device over another was not due to differences in performance. However, by utilising this post hoc approach, it remained unclear when a child's preference for an AAC option might first emerge and why children might prefer one AAC option over another. It was also unclear whether preferences led to improved therapeutic outcomes. By incorporating preference assessments into the initial stages of the intervention, the current study was able to determine when preferences began to emerge and whether preference might have influenced acquisition and maintenance.

Along these lines, our results indicated that for three of the four participants (Tom, Zac, and Eli), preference was evident during the early stages of intervention and appeared to remain stable during the study. For David, however, preference seemed to change as he gained proficiency with each communication option. Specifically, David learned MS rapidly and initially demonstrated a preference for this communication option. However, after learning to use the iPod ${ }^{\circledR}$-based SGD, he then started to choose this communication option.

The results also suggested that participants were more proficient at using their preferred AAC option or perhaps it should be said that they preferred the option with which they were more proficient. Furthermore, maintenance of newly acquired communication skills was better when participants used their preferred option. These results provide the first empirical evidence to suggest that allowing individuals with DD to determine which communication option they would like to use might positively influence progress in learning to communicate. These findings support the argument by Soto et al. (1993) that AAC acquisition is interconnected with choice-making and preference.

However, similar to the literature pertaining to general choice-making and assessment of preference in individuals with DD (e.g., Stafford, Alberto, Fredrick, Heflin, \& Heller, 2002), preference did change over time for one participant (David), verifying the idea that preference should be reassessed at regular intervals. Because preferences can vary it has also been suggested that an individual's preference, or the 'magnitude of preference', for one AAC option over another should be interpreted with caution (van der Meer, Sigafoos, et al., 2011). Van der Meer, Sigafoos, et al. raised the question: "How many choice opportunities are required to demonstrate a preference for one mode of communication over another?" (p. 1429). There is not yet any firm empirical basis for setting the percentage of selections from which an item can be considered preferred. Future research should investigate this issue.

Results from the present study suggest that the choice-making paradigm (e.g., Sigafoos, 1998) utilized in the preference research to date might be effectively extended to assess preferences for aided (SGD) versus unaided (MS) AAC. Prior to this study, it was unknown whether using a photo/diagram of a person signing would be a viable means of representing the MS option. David did select the MS option the majority of the time suggesting that at least one participant did understand that the photo of the trainer signing represented the MS option, enabling him to indicate a preference for an unaided mode of AAC. Future research is required to further investigate the validity of this approach, especially considering it could be argued that the SGD and MS options were not functionally equivalent. While the SGD option included symbols for SOCIAL INTERACTION, SNACK, and PLAY, the MS option only had two photos to represent SNACK and PLAY. Furthermore, in order to compare two conditions on symbol learning, it is crucial to equate the sets of symbol-referent associations for SGD and MS for the degree of 
iconicity and for receptive understanding of the referent (Schlosser, 2003b). Linked to this, it has been argued that the response effort required for each communication system may influence preference and intervention outcomes (Ringdahl et al., 2009; Winborn-Kemmerer et al., 2009). Although signing to request to eat/play was considered comparable to using the SGD, this decision was based only upon informal methods of creating equivalency in terms of performance difficulty (Schlosser, 2003b). However, the two systems might not be comparable in terms of cognitive demands. Indeed, it has been argued that graphic symbols of the type used with the SGD are less demanding on working memory because only recognition is needed, whereas the MS option requires the use of recall memory (Iacono et al., 1993). This could be one possible reason why some participants might demonstrate a preference for SGD over MS (Iacono \& Duncum, 1995).

A further potential problem of the current study is that a MS response could be emitted during SGD sessions, but not vice versa because the SGD was not available during MS sessions. This could have meant that correct MS responses were put on a more intermittent schedule (correct MS responses resulted in reinforcement only some of the time, while correct SGD responses resulted in reinforcement $100 \%$ of the time) relative to correct SGD responses, thereby weakening the teaching contingencies and potentially influencing both preferences and rapidity of acquisition. Despite this possibility, the majority of participants (David, Tom, and Eli) did learn to use both MS and SGD for functional communication suggesting that any disruption from intermittent reinforcement of the MS option was minimal and short-lived. Overall, the results of the intervention phase, showing acquisition of SGD and MS use in most cases, are consistent with previous research (Rispoli et al., 2010; van der Meer \& Rispoli, 2010; Wendt, 2009) showing that systematic instructional procedures, including graduated guidance, time delay, and contingent reinforcement procedures, can be effectively applied to teach requesting for preferred stimuli using both unaided and aided AAC (Duker et al., 2004).

While David and Eli demonstrated rapid acquisition of both the SGD and MS, Zac failed to show any progress during intervention for MS and struggled to learn the SGD. Adaptation of the instructional techniques included the introduction of mass training trials. With this procedure in place Zac rapidly learnt to use the SGD, but failed to learn MS. His lack of progress with the MS option did not appear to be a motivational issue in that he did consistently select and play with preferred stimuli. In addition, it did not appear to be due to an inability to physically perform the hand gestures required to make the manual sign because he did independently request to play using MS on a number of occasions. Anecdotally, when Zac was prompted to make the sign for PLAY he also often attempted to verbalize the word play. As mentioned earlier, treatment outcomes for learning alternative forms of communication may vary if the new response topography is more demanding than the individual's original form of communication (Winborn-Kemmerer et al., 2009). Perhaps learning the sign for PLAY required significantly more effort for Zac than attempting to verbalize the request.

Adaptations to the instructional procedures, including least-to-most prompting and later an increased time-delay with graduated guidance and differential reinforcement did lead to acquisition of the MS option for Tom. This highlights the importance of not only implementing well established teaching procedures, but also modifying these techniques to suit the needs of each individual (Linscheid, 1999). Results also support preliminary evidence in the instructional literature on teaching the use of a new iPod ${ }^{\circledR}$-based SGD. Similar to this previous research (Kagohara et al., 2010; van der Meer, Sigafoos, et al., 2011), two of the participants (David and Zac) in the current study did show some initial difficulty in learning to touch the target symbols on the SGD with sufficient finesse to activate speech output. Fortunately, with practice, both David and Zac became proficient with using the iPod ${ }^{\circledR}$-based SGD.

While using the behavioral strategies described above often leads to rapid acquisition of functional communication, several problems can emerge following acquisition of an initial requesting repertoire (Sigafoos, Ganz, O’Reilly, \& Lancioni, 2008). One such problem, which was evident for David and Eli, was the development of preservative requesting, which refers to making repeated requests, even after being informed to wait for the requested item or when the item is not being offered (Sigafoos et al., 2008). David initially used MS repeatedly even during SGD sessions, which Cannella-Malone et al. (2009) suggested could be an indication of preference for a particular AAC device. However, we ignored signing when it was used in an incorrect context and this appeared to be a uselful part of the training program for David because he eventually developed proficiency with the SGD and his preservative signing diminished. He also began to choose the SGD during the device preference assessments. Eli, on the other hand, developed a tendency to repeatedly press the icons on the SGD resulting in constant voice-output. Observations of this behavior suggested that this might have been automatically reinforced by the resulting synthesized speech output.

Because Eli repeatedly pressed non-target symbols on the SGD, it could be argued that he did not have good symbol discrimination abilities. However, for the most part Eli and the other participants did correctly request to play (eat) the items that were being offered, indicating good symbol discrimination abilities. Still, future research could implement additional manipulations to control for item-biased or position-biased responding, such as the procedures implemented by van der Meer, Kagohara, et al. (2011). They shifted the orientation of the iPod ${ }^{\circledR}$-based SGD and alternated offering only toys or only snacks across sessions to test for the relevant symbol discriminations.

The results of the present study are limited in several ways and should be interpreted with caution. In line with previous research on assessing preferences for different AAC options (see van der Meer, Sigafoos, et al., 2011), the present study focused on teaching a single communicative function (i.e., requesting). It therefore remains unclear whether preference will influence learning of other communication skills. Furthermore, because we used a verbal prompt (Let me know if you want something.) to initiate requesting opportunities, it is possible that the participant's responses were at least partially under the control of the speech of the communication partner. This means that the participants might not have acquired the ability to request items more spontaneously. Future interventions could be improved by teaching more spontaneous communication 
(Sigafoos, Drasgow, et al., 2009). The fact that only a limited communicative repertoire was taught and that generalization data to other communicative forms, contexts, and people were not collected are further limitations. Lastly, preferences across a wider range of AAC options (e.g., PECS, SGD, and MS) have yet to be investigated. Since these are the three main AAC modes taught to children with DD, a study that simultaneously compared all three options in terms of acquisition, preference and maintenance would seem relevant and timely.

\section{Conflict of interest}

The authors report no conflicts of interest. The authors alone are solely responsible for the content and writing of this paper.

\section{Acknowledgments}

Support for this research was provided from the New Zealand Government through the Marsden Fund Council, administered by the Royal Society of New Zealand; and by Victoria University of Wellington, The University of Canterbury, and The New Zealand Institute of Language, Brain \& Behaviour.

\section{References}

Adkins, T., \& Axelrod, S. (2001). Topography- versus selection-based responding: Comparison of mand acquisition in each modality. The Behavior Analyst Today, 2, $259-266$.

Beck, A., Stoner, J., Bock, S., \& Parton, T. (2008). Comparison of PECS and the use of a VOCA: A replication. Education and Training in Developmental Disabilities, 43, $198-216$.

Beukelman, D., \& Mirenda, P. (2005). Augmentative and alternative communication: Supporting children and adults with complex communication needs (3rd ed.). Baltimore: Paul H Brookes Publishing Co.

Bock, S., Stoner, J., Beck, A., Hanley, L., \& Prochnow, J. (2005). Increasing functional communication in non-speaking preschool children: Comparison of PECS and VOCA. Education and Training in Developmental Disabilities, 40, 268-278.

Bondy, A., \& Frost, L. (2001). The Picture Exchange Communication System. Behavior Modification, 25, 725-744.

Cannella-Malone, H., DeBar, R., \& Sigafoos, J. (2009). An examination of preference for augmentative and alternative communication devices with two boys with significant intellectual disabilities. Augmentative and Alternative Communication, 25, 262-273.

DeLeon, I., \& Iwata, B. (1996). Evaluation of a multiple-stimulus presentation format for assessing reinforcer preferences. Journal of Applied Behavior Analysis, 29, 519-532.

Duker P., Didden R., \& Sigafoos J., (2004). One-to-one training: Instructional procedures for learners with developmental disabilities. Austin: Pro-Ed.

Goldstein, H., (2002). Communication intervention for children with autism: A review of treatment efficacy. Journal of Autism and Developmental Disorders, 32, 373-394.

Green, V., Sigafoos, J., Didden, R., O’Reilly, M., Lancioni, G., Ollington, N., et al. (2008). Validity of a structured interview protocol for assessing children's preferences. In P. Grotewell \& Y. Burton (Eds.), Early childhood education: Issues and developments (pp. 87-103). New York: Nova Science Publishers.

Gregory, M., DeLeon, I., \& Richman, D. (2009). The influence of matching and motor-imitation abilities on rapid acquisition of manual signs and exchange-based communication responses. Journal of Applied Behavior Analysis, 42, 399-404.

Iacono, T., \& Duncum, J. (1995). Comparison of sign alone and in combination with an electronic communication device in early language intervention: Case study. Augmentative and Alternative Communication, 11, 249-259.

Iacono, T., Mirenda, P., \& Beukelman, D. (1993). Comparison of unimodal and multimodal AAC techniques for children with intellectual disabilities. Augmentative and Alternative Communication, 9, 83-94.

Iversen, P. (2007). The informative pointing method. Available from www.strangeson.com (accessed 16.06.2010).

Kagohara, D., van der Meer, L., Achmadi, D., Green, V., O’Reilly, M., Mulloy, A., et al. (2010). Behavioral intervention promotes successful use of an iPod-based communication device by an adolescent with autism. Clinical Case Studies, 9, 328-338.

Kennedy, C. H., (2005). Single-case designs for educational research. Boston: Pearson Education Inc.

Lancioni, G., O'Reilly, M., Cuvo, A., Singh, N., Sigafoos, J., \& Didden, R. (2007). PECS and VOCAs to enable students with developmental disabilities to make requests: An overview of the literature. Research in Developmental Disabilities, 28, 468-488.

Linscheid, T., (1999). Commentary: Response to empirically supported treatments for feeding problems. Journal of Pediatric Psychology, 24, 215-216.

Makaton New Zealand/Aotearoa. (1998-1999). Sign illustrations for Makaton core vocabulary. Auckland: Westprint.

Mirenda, P., (2003). Toward functional augmentative and alternative communication for students with autism: Manual signs, graphic symbols, and voice output communication aids. Language, Speech E Hearing Services in Schools, 34, 203-216.

National Research Council. (2001). Educating children with autism. Washington, DC: Henry (Joseph) Press.

Peeters, T., \& Gillberg, C. (1999). Autism: Medical and educational aspects. London: Whurr.

Ringdahl, J., Falcomata, T., Christensen, T., Bass-Ringdahl, S., Lentz, A., Dutt, A., et al. (2009). Evaluation of a pre-treatment assessment to select mand topographies for functional communication training. Research in Developmental Disabilities, 30, 330-341.

Rispoli, M., Franco, J., van der Meer, L., Lang, R., \& Carmargo, S. (2010). The use of speech generating devices in communication interventions for individuals with developmental disabilities: A review of the literature. Developmental Neurorehabilitation, 13, 276-293.

Rotholz, D., Berkowitz, S., \& Burberry, J. (1989). Functionality of two modes of communication in the community by students with developmental disabilities: A comparison of signing and communication books. Journal of the Association for Persons with Severe Handicaps, 14, $227-233$.

Schlosser, R., (2003a). The efficacy of augmentative and alternative communication: Toward evidence-based practice. San Diego: Academic Press.

Schlosser, R., (2003b). Single-subject designs. In Schlosser, R. (Ed.). The efficacy of augmentative and alternative communication: Toward evidence-based practice. (pp.85-143). .

Schlosser, R., \& Blischak, D. (2001). Is there a role for speech output in interventions for persons with autism? Focus on Autism E' Other Developmental Disabilities, $16,170-178$.

Schlosser, R., \& Wendt, O. (2008a). Augmentative and alternative communication intervention for children with autism. In J. Luiselli, D. Russo, W. Christian, \& S. Wilczynski (Eds.), Effective practices for children with autism: Educational and behavioral support interventions that work (pp. 325-389). Oxford: Oxford University Press.

Schlosser, R., \& Wendt, O. (2008b). Effects of augmentative and alternative communication intervention on speech production in children with autism: A systematic review. American Journal of Speech-Language Pathology, 17, 212-230.

Sennott, S., \& Bowker, A. (2009). Autism, AAC and Proloquo2Go. Perspectives on Augmentative and Alternative Communication, 18, $137-145$.

Sigafoos, J., (1998). Choice making and personal selection strategies. In J. Luiselli \& M. Cameron (Eds.), Antecedent control: Innovative approaches to behavioral support (pp. 187-221). Baltimore: Paul H. 
Sigafoos, J., (2006). Self-determination: Can we let the child determine the best treatment? Pediatric Rehabilitation, 9, 1-2.

Sigafoos, J., \& Drasgow, E. (2001). Conditional use of aided and unaided AAC: A review and clinical case demonstration. Focus on Autism Er Other Developmental Disabilities, 16, 152-161.

Sigafoos, J., Drasgow, E., Duker, P., O’Reilly, M., Lancioni, G., \& Reichle, J. (2009). General intervention approaches for teaching speech and its prerequisites. In A. Fitzer \& P. Sturmey (Eds.), Language and autism: Applied behavior analysis, evidence, and practice (pp. 109-133). Austin, Texas: Pro-Ed.

Sigafoos, J., Drasgow, E., \& Schlosser, R. (2003). Strategies for beginning communicators. In R. Schlosser (Ed.), The efficacy of augmentative and alternative communication: Toward evidence-based practice (pp. 323-346). San Diego: Academic Press.

Sigafoos, J., Ganz, J., O'Reilly, M., \& Lancioni, G. (2008). Evidence-based practice in the classroom: Evaluating a procedure for reducing perseverative requesting in an adolescent with autism and severe intellectual disability. Australasian Journal of Special Education, 32, 55-65.

Sigafoos, J., Green, V., Payne, D., Son, S., O’Reilly, M., \& Lancioni, G. (2009). A comparison of picture exchange and speech-generating deivces: Acquisition, pereference and effects on social interaction. Augmentative and Alternative Communication, 25, 99-109.

Sigafoos, J., \& Iacono, T. (1993). Selecting augmentative communication devices for persons with severe disabilities: Some factors for educational teams to consider. Australian and New Zealand Journal of Developmental Disabilities, 18, 133-146.

Sigafoos, J., O'Reilly, M., Ganz, J., Lancioni, G., \& Schlosser, R. (2005). Supporting self-determination in AAC interventions by assessing preference for communication devices. Technology and Disability, 17, 1-11.

Son, S., Sigafoos, J., O’Reilly, M., \& Lancioni, G. (2006). Comparing two types of augmentative and alternative communication systems for children with autism. Pediatric Rehabilitation, 9, 389-395.

Soto, G., Belfiore, P., Schlosser, R., \& Haynes, C. (1993). Teaching specific requests: A comparative analysis of skill acquisition and preference using two augmentative and alternative communication aids. Education and Training in Mental Retardation, 28, 169-178.

Sparrow, S., Cicchetti, D., \& Balla, D. (2005). Vineland-II adaptive behavior scales (2nd ed.). Minneapolis: Pearson.

Stafford, A., Alberto, P., Fredrick, L., Heflin, L., \& Heller, K. (2002). Preference variability and the instruction of choice making with students with severe intellectual disabilities. Education and Training in Mental Retardation and Developmental Disabilities, 37, 70-88.

Tincani, M., (2004). Comparing the picture exchange communication system and sign language training for children with autism. Focus on Autism E Other Developmental Disabilities, 19, 152-163.

van der Meer, L., Kagohara, D., Achmadi, D., Green, V., O’Reilly, M., Lancioni, G., et al. (2011). Teaching functional use of an iPod-based speech-generating device to individuals with developmental disabilities. Journal of Special Education Technology, 26, 1-12.

van der Meer, L., \& Rispoli, M. (2010). Communication interventions involving speech-generating devices for children with autism: A review of the literature. Developmental Neurorehabilitation, 13, 294-306.

van der Meer, L., Sigafoos, J., O'Reilly, M., \& Lancioni, G. (2011). Assessing preferences for AAC options in communication interventions for individuals with developmental disabilities: A review of the literature. Research in Developmental Disabilities, 32, 1422-1431.

Wendt, O., (2009). Research on the use of manual signs and graphic symbols in autism spectrum disorders: A systematic review. In P. Mirenda \& T. Iacono (Eds.), Autism spectrum disorders and AAC (pp. 83-140). Baltimore: Paul H.

Winborn-Kemmerer, L., Ringdahl, J., Wacker, D., \& Kitsukawa, K. (2009). A demonstration of individual preference for novel mands during functional communication training. Journal of Applied Behavior Analysis, 42, 185-189. 


\section{APPENDIX F}

van der Meer, L., Didden, R., Sutherland, D., O'Reilly, M., Lancioni, G., \& Sigafoos, J. (2012). Comparing three augmentative and alternative communication modes for children with developmental disabilities.

Journal of Developmental and Physical Disabilities. doi: 10.1007/s10882012-9283-3 


\title{
Comparing Three Augmentative and Alternative Communication Modes for Children with Developmental Disabilities
}

\author{
Larah van der Meer • Robert Didden • \\ Dean Sutherland • Mark F. O'Reilly • \\ Giulio E. Lancioni • Jeff Sigafoos
}

(C) Springer Science+Business Media, LLC 2012

\begin{abstract}
We compared acquisition, maintenance, and preference for three AAC modes in four children with developmental disabilities (DD). Children were taught to make general requests for preferred items (snacks or play) using a speechgenerating device (SGD), picture-exchange (PE), and manual signs (MS). The effects of intervention were evaluated in a multiple-probe across participants and alternatingtreatments design. Preference probes were also conducted to determine if children would choose one AAC mode more frequently than the others. During intervention, all four children learned to request using PE and the SGD, but only two also reached criteria with MS. For the AAC preference assessments, three participants chose the SGD most frequently, while the other participant chose PE most frequently. The results suggest that children's preference for different AAC modes can be assessed
\end{abstract}

L. van der Meer · J. Sigafoos

Victoria University of Wellington, Wellington, New Zealand

R. Didden

Radboud University Nijmegen, Nijmegen, The Netherlands

D. Sutherland

University of Canterbury, Christchurch, New Zealand

M. F. O'Reilly

The Meadows Center for the Prevention of Educational Risk, University of Texas at Austin, Austin, TX, USA

G. E. Lancioni

University of Bari, Bari, Italy

L. van der Meer $(\bowtie)$

School of Educational Psychology and Pedagogy, Victoria University of Wellington, PO Box 17-310, Karori, 6147 Wellington, New Zealand

e-mail: larah.vandermeer@vuw.ac.nz 
during the early stages of intervention and that their preferences may influence acquisition and maintenance of AAC-based requesting responses.

Keywords Augmentative and alternative communication - Developmental disabilities $\cdot$ Manual sign $\cdot$ Picture-exchange $\cdot$ Speech-generating devices

Children with developmental disabilities (DD) often present with significant deficits in speech and language development. To enable these children to communicate, intervention typically involves teaching the use of augmentative and alternative communication (AAC; Beukelman and Mirenda 2005; Schlosser 2003a). Three common AAC modes are speech-generating devices (SGDs; Lancioni et al. 2007), picture exchange (PE) or more specifically the Picture Exchange Communication System (PECS; Bondy and Frost 1994; Bondy and Frost 2001), and manual signing (MS; Lloyd, Fuller, \& Arvidson, 1997). Data indicate that all three of these AAC modes can be successfully taught to children with DD (Flippin et al. 2010; Goldstein 2002; Hart and Banda 2010; Lancioni et al. 2007; Preston and Carter 2009; Rispoli et al. 2010; Schlosser and Wendt 2008a; Schlosser and Wendt 2008b; Sulzer-Azaroff et al. 2009; Tien 2008; van der Meer and Rispoli 2010; Wendt 2009).

While there is evidence to support the use of each of these three AAC modes, debate continues regarding the relative efficacy of these three options for individuals with DD (Mirenda 2003; Schlosser and Sigafoos 2006). To shed some empirical light on this debate, one can turn to the results of several studies that have compared acquisition of PE versus MS (Adkins and Axelrod 2001; Gregory et al. 2009; Rotholz et al. 1989; Tincani 2004), as well as SGDs versus PE (Beck et al. 2008; Bock et al. 2005). Few studies have compared SGD and MS (Iacono and Duncum 1995; Iacono et al. 1993; van der Meer et al. 2012). One consistent finding from these comparison studies is that there seems to be no large or consistent differences in terms how effectively and efficiently the compared AAC modes can be taught to participants with DD.

In light of such evidence, Sigafoos et al. (2003) proposed that clinicians might profitably examine the extent to which individuals show a preference for using one AAC mode over another. Along these lines, a number of studies have assessed preferences for different AAC modes (Cannella-Malone et al. 2009; Sigafoos et al. 2009; Sigafoos et al. 2005; Son et al. 2006; Soto et al. 1993; Winborn-Kemmerer et al. 2009). In these studies, the participant's preference for using one mode of communication over another was assessed using a structured choice-making arrangement (Sigafoos 1998). Specifically, participants were taught to use two different AAC options (e.g., SGD and PE) for functional communication (e.g., requesting a snack) and were then given the opportunity to choose which AAC mode to use during subsequent requesting opportunities. A communication system was considered preferred when it was consistently chosen more often than the other option.

A systematic review of this AAC-preference literature (van der Meer et al. 2011b) indicated that individuals with DD do in fact often demonstrate a preference for using (choosing) one AAC mode over another. However, this existing group of studies is limited in a number of ways. First, preference assessments were only undertaken after participants already learned to use each communication option. This post-hoc 
approach effectively prevented an evaluation of whether preference can be incorporated into, and identified during, the initial stages of AAC intervention. Second, only one study (Soto et al. 1993) collected maintenance data. It is therefore unclear whether employing preferred AAC systems improves intervention outcomes in terms of long-term maintenance of newly acquired communication skills or if preferences remain stable over time. Third, the majority of studies reviewed only assessed preference between two (SGD and PE) AAC modes (van der Meer et al. 2011b). Although one study (Iacono and Duncum 1995) compared the use of MS versus SGD and inferred a preference for one over the other based upon effectiveness of use, none of the studies assessed preferences among all three of these commonly used AAC modes (i.e., SGD, PE, and MS).

Along those lines, van der Meer et al. (2012) addressed each of these limitations. They compared acquisition of augmented requesting responses using an $\mathrm{PPod}^{\circledR}$-based SGD versus MS. Preference assessments were implemented throughout the intervention in order to determine whether four participants (with DD, aged 5 to 10 years) made relatively greater progress with the SGD or MS. Results showed that all four participants learned to request preferred objects. Three participants exhibited a preference for using the SGD, while one participant demonstrated a preference for using MS. Additionally, participants were more proficient at using their preferred AAC option and maintenance of communication skills was better with their preferred option.

The present study was designed to systematically replicate and extend the work of van der Meer et al. (2012) by including more participants and comparing acquisition of, and preference for, SGD, MS, and PE. Based on the results of the van der Meer et al. (2012) study, we hypothesized that the four children participating in the current study would show a preference for using one AAC mode over the other two, that these preferences would vary across children, and that the children would learn to request preferred stimuli more quickly with their most preferred AAC mode.

\section{Method}

\section{Participants}

Four children were recruited due to their severely limited speech from a childcare center for children with DD. All four participants met the following criteria: (a) diagnosis of intellectual/developmental disability or autism spectrum disorder (ASD), (b) less than 18 years of age, (c) very limited or no communication skills as determined by an age equivalency of 2 years or less on the Communication Domain of the Vineland - Z, Dutch edition (Sparrow et al. 2003), (d) no auditory or visual impairments that would interfere with the use of AAC, and (e) sufficient motor skills to operate/perform the motor actions required to use each of the three AAC modes.

Joe was a 12-year-old male diagnosed with ASD. On the Vineland - Z (Sparrow et al. 2003), Joe received age equivalencies of 1:2 (years:months) for communication, 1:4 for daily living skills, and 1:1 for socialization. Joe did not have any spoken language, but made sounds that were presumed to be his way of expressing how he 
was feeling (e.g., happy versus sad). He also appeared to communicate his wants and needs by taking a person's hand and leading them to an object. Joe's teachers used a picture communication board to explain routine activities for the day (toilet, food and drink, gym, bus, free choice, and outside play). Teachers had also introduced him to MS for receptive language. He did not have any prior experience with SGDs, PE, or MS as a communication mode for requesting access to preferred objects. Joe's fine and gross motor skills appeared to be adequate for his chronological age. He demonstrated frequent stereotypical and repetitive behaviors, such as flapping small toys and pieces of paper in front of his eyes.

Sam was a 6-year-old male diagnosed with childhood disintegrative disorder and intellectual disability. He received age equivalencies on the Vineland - Z (Sparrow et al. 2003) of 1:2, 1:8, and 0:11 (years:months) for the communication, daily living skills, and socialization domains, respectively. Sam did not have any spoken language. He would take a person's hand and lead them to objects to seemingly express his wants and needs. He also made sounds that were thought to be indications of disapproval. His teachers tried to use MS when communicating with Sam, but he did not appear to show any interest in using MS to make requests. As was the case for Joe, Sam did not have any prior experience with SGDs, PE, or MS as a communication mode for requesting access to preferred objects.

Saskia was a 10-year-old female with Angelman syndrome. She received an age equivalency of 1:4 (years:months) for the communication domain and 1:0 for both the daily living skills and socialization domains of the Vineland $-\mathrm{Z}$ (Sparrow et al. 2003). Saskia was able to speak several single words, mostly in the form of echolalia. She would often take people's hands to seemingly direct them to what she wanted, to open things, or to clap for her. Saskia had some experience with MS and was able to produce the signs for FINISHED and OPEN. She had no further experience with other forms of AAC. She exhibited difficulty with social interaction and engaged in stereotypic and repetitive behaviors. For example, she seemed more interested in adults and, although she appeared to enjoy watching other children play, she would push them away if they approached her. She also flapped keys and other objects in front of her eyes.

Nicky was a 13-year-old female diagnosed with pervasive developmental disorder not otherwise specified (PDD-NOS). Nicky received age equivalencies on the Vineland $-\mathrm{Z}$ (Sparrow et al. 2003) of 1:3, 1:1, and 1:3 (years:months) for the communication, daily living skills, and socialization domains respectively. She was able to verbalize several single words, but generally only made babbling sounds. She had previously received MS training and was able to produce several signs, including OPEN, EAT, and CIRCLE TIME. Nicky appeared to understand symbols from her daily picture communication book and from routine activities. She was able to match picture cards and had received 1 year of PECS training, but reportedly had made little progress. She had received no further training in the use of AAC for requesting preferred objects. Nicky was able to maintain good eye contact and seek social contact, but she was said to be very excitable and did not seem to understand social boundaries. She was not able to play cooperatively with other children. Nicky often cried in an apparent attempt to gain attention. Nicky's fine motor skills were adequate for her developmental level. She was able to walk on her own, although she was hypotonic. 
Setting and Intervention Context

Participants were recruited from a Dutch childcare center for children with DD. The procedures related to this study were conducted in a small therapy room across the hall from the children's main classrooms. Sessions occurred during morning and afternoon snack/leisure activities. The procedures were implemented in a one-to-one context consisting of the trainer (first author) and one participant at a time. All instructions/interactions with the participants and responses programmed on the AAC systems were in the Dutch language.

\section{Preferred Stimuli}

Stimuli that the children seemed to prefer, and which would be appropriate for them to request during a snack or leisure activity, were identified by a systematic two-stage stimulus preference assessment (Green et al. 2008). Stage 1 of the preference assessment involved an indirect assessment in which teachers were asked to list foods, sensory stimuli, and toys that the participants appeared to enjoy and would be appropriate for the intervention.

For Stage 2, three to six of the most preferred food or play stimuli were then selected for a direct stimulus assessment. The direct preference assessment for Joe focused on identifying preferred foods because his intervention occurred during a snack activity and his teachers reported that he seemed to be highly motivated by snack foods. The direct preference assessment for Sam, Saskia, and Nicky, in contrast, focused on identifying preferred toys because their intervention occurred during a play activity and their teachers had concerns about using food reinforcers. Stage 2 involved the simultaneous presentation of multiple items, without replacement (DeLeon and Iwata 1996; Duker et al. 2004). Each participant was presented with an array of items from Stage 1 (random placement) and allowed to select one item. Items were not replaced once they had been selected, thereby eliminating the chance of the participant choosing only one or a few items, as well as allowing the trainer to develop a rank order of items in terms of preference. The top three food items for Joe and top three play items for Sam, Saskia, Nicky were identified by calculating a rank order of the percentage of times that the stimuli were selected. Across two to three sessions, each item was offered a total of nine times. Rank orders were calculated using the formula: Number of Selections/Number of Offers x $100 \%$.

Preferred stimuli for Joe included 'skittles' lollies (75 \%), 'tumtum' lollies (33\%), and potato chips (32\%). Preferred stimuli for Sam included a puzzle (60\%), venting ball (36 \%), and windmill (33\%). Preferred stimuli for Saskia included a musical toy (82\%), keys and lanyard (39\%), and bubbles (31\%). Preferred items for Nicky included a tea set $(69 \%)$, dolls $(56 \%)$, and a mirror (32\%).

\section{Speech-Generating Device (SGD)}

Participants were taught to request preferred toys or snacks using an Apple iPod Touch $^{\circledR}$ with Proloquo2Go ${ }^{\mathrm{TM}}$ software. The iPod was placed inside an iMainGo ${ }^{\circledR} 2$ speaker case to increase sound amplification. The iPod was configured to show a single page containing two graphic symbols $(2.5 \times 2.5 \mathrm{~cm})$, representing requests for 
SNACKS and PLAY. The messages were programmed in Dutch. Touching each symbol activated corresponding synthetic speech-output (i.e., "I want something to eat.", and "I want to play.").

\section{Picture Exchange (PE)}

Participants were also taught to request their preferred toys or snacks using PE. Three $(6 \times 6 \mathrm{~cm})$ symbols from the PECS 2009 Dictionary (Pyramid Educational Products 2009 ) were affixed with Velcro ${ }^{\mathrm{TM}}$ to a $19 \times 13 \mathrm{~cm}$ card. One symbol contained a colored line drawing showing two hands reaching out and the words I WANT. The second symbol consisted of a colored line drawing of various different toys and the words TO PLAY. The third symbol consisted of a colored line drawing of various snack items and the message SOMETHING TO EAT. All words were written in Dutch. The symbols were randomly allocated to the six $(6 \times 6 \mathrm{~cm})$ panels of the card.

\section{Manual Signing (MS)}

Participants were taught to request their preferred toys or snacks using signs from the Dutch sign language system for children (Nederlands Gebarencentrum 2006). Participants were taught the sign for SNACK or PLAY. The MS option was represented by a laminated photograph $(15 \times 8 \mathrm{~cm})$ of the trainer making the hand formations for the signs for SNACK and PLAY.

\section{Response Definitions and Measurement}

For SGD use, correct responding was defined as independently (without a gestural or verbal prompt) touching the symbol on the screen of the SGD to activate the corresponding speech output in exchange for a desired item from the trainer. MS was defined as independent (without a gestural or verbal prompt) hand gestures to produce correct signs in exchange for a desired item from the trainer. For PE, participants were required to independently (without gestural or verbal prompt) place the I WANT and corresponding ( $S N A C K S$ or PLAY) symbols - depending on whether they were requesting snacks or play - onto the two panels $(6 \times 6 \mathrm{~cm})$ provided on a separate $(21 \times 7 \mathrm{~cm})$ card in exchange for the desired item from the trainer.

The percentage of correct responses (requests) was calculated for each session. Each session consisted of 10 offers to request snack or play items. The SGD target response for Joe was touching the $S N A C K$ symbol on the SGD to activate the message "I want a snack." His target response for PE was placing the I WANT and SOMETHING TO EAT symbols on the separate card. The PLAY symbol (SGD and $\mathrm{PE}$ ) was intended as a distracter. Joe's target response for MS was to produce the manual sign for SNACK. The PLAY sign (on the laminated card) was intended as a distractor. The SGD target response for Sam, Saskia, and Nicky was touching the PLAY symbol on the SGD to activate the message "I want to play." Their target response for PE was placing the I WANT and TO PLAY symbols on the separate card. The SNACK symbol (SGD and PE) was intended as a distracter. The MS target response for Sam, Saskia, and Nicky was to produce the manual sign for PLAY. The SNACK sign (on the laminated card) was intended as a distractor. 


\section{Experimental Design}

The study included the following phases arranged in a multiple-probe across participants design (Kennedy 2005): Baseline, Intervention, Preference Assessment (throughout Intervention, Post-Intervention, and Follow-Up), Post-Intervention, and Follow-Up. An alternating-treatments design was embedded within each phase of the multiple-probe to compare children's performance with the SGD, PE, and MS options.

\section{Session Schedule}

Two to four sessions were conducted 5 days per week. Each session lasted about $10 \mathrm{~min}$ and consisted of 10 discrete trials. The AAC option available (i.e., SGD, PE, or MS) was counterbalanced across sessions to prevent order effects (Kennedy 2005). For all sessions, the participant and trainer were seated next to each other at a table with one or two additional reliability and procedural integrity observers seated nearby.

Once a participant showed an increase in requesting behavior above the level established in baseline for three consecutive sessions with at least one of the AAC options, training commenced with the next participant. Training was first provided to Joe, then Sam, Saskia, and finally Nicky. Training was provided in this order in accordance with results from the baseline phase (i.e., the participant with the most stable baseline commenced intervention first). Once a participant reached criterion for one AAC condition (i.e., $80 \%$ correct requesting across three consecutive sessions for each AAC option), maintenance probes were initiated with that system while the other communication systems continued to be taught using the intervention procedures. One maintenance session with the acquired AAC device was conducted after three sessions with each of the AAC options still being taught.

\section{Procedures}

Because participants were considered to be at the beginning stages of AAC intervention, they were taught to make general requests for either snacks or toys from which they could select one highly preferred item after each request. However, to ensure some level of symbol discrimination, the distractor symbols/signs were included on the AAC options. If participants requested a snack when they were undertaking training to request a toy or vice versa, the trainer explained: We are learning to request toys (snack) at the moment, you can request a toy (snack) another time. It was considered natural to provide them with this feedback when they activated the nontarget symbols/produced the non-target signs, but not to reinforce it with preferred tangibles. Similarly, producing MS to request snacks or toys during SGD or PE sessions was ignored in order to bring the use of each device under stimulus control.

Baseline During baseline, a tray containing three different snack (play) items was placed on the table in view, but out of the participants' reach. The SGD, PE, and MS option (represented by the photograph of the trainer making the two signs) were randomly placed on different sides of the table for each baseline session. Each session 
involved 10 discrete trials for snacks or toys. The session began with the trainer telling the participant: Here is a tray of snacks (toys), let me know if you want something. After $10 \mathrm{~s}$, the trainer moved the tray within reach and allowed the participant to take one item. This 10-s fixed time schedule of reinforcement was provided to ensure continued motivation to participate in sessions. When offering snacks, participants were allowed to select one item from the tray, which was then replenished before the next offer. When offering the tray of toys, participants were allowed to select one toy and play with it for approximately $30 \mathrm{~s}$ before it was returned to the tray. SGD, PE, and MS responses were recorded, but had no programmed consequences.

Intervention This phase was conducted in a discrete trial format until participants reached criterion (i.e., $80 \%$ correct requesting across three consecutive sessions for each AAC option). The SGD, PE, or the MS option was placed on the table (counterbalanced across trials) within reach of the child in accordance with the alternating treatments design. Each trial consisted of the trainer pointing to a tray of snacks (toys) and saying: Here's a tray of snacks (toys). Let me know if you want something. Training involved a 10-s time delay between the verbal cue (i.e., Let me know if you want something.) and the use of graduated guidance to prompt a correct request. Graduated guidance involved use of the least amount of physical guidance necessary to ensure the child made a correct request, while simultaneously explaining the required response (e.g., Press PLAY to ask to play with a toy. or Move your hand to your mouth to make the sign for EAT. or Put the I WANT and PLAY pictures on the velcro strips). Immediately after the child had used the SGD to produce the correct synthesized speech output, or had placed the appropriate symbols on the Velcro strips, or had made the correct manual sign, the trainer moved the tray containing the snacks (or toys) within reach of the participant. The participant was allowed to select one item from the tray and consume the chosen snack or play with the chosen toy for about $30 \mathrm{~s}$. After this, the next trial was initiated.

Procedural Modifications Joe did not reach criterion for each communication system during the initial intervention phase so he received a modified intervention. The modification was developed in response to what appeared to be a problem in teaching him to discriminate among the different symbols. Therefore, the PLAY symbol was removed from the SGD and PE options (see the 1 Symbol phase of Fig. 1). The only icon displayed on the SGD screen was therefore the SNACK icon, which was also enlarged to fit the entire screen. Joe was only required to press this icon to activate the voice-output in order to make a correct request for a preferred snack item. For the PE option, he no longer had to discriminate between the SNACK and PLAY symbols. For a correct request he had to place the I WANT and SOMETHIG TO EAT symbols onto the two locations provided on the separate card in exchange for the desired item from the trainer.

Sam also failed to learn how to use the SGD and MS communication options during the initial intervention sessions. Therefore, for SGD and for MS (see the $20 \mathrm{~s}$ Time Delay \& Differential Reinforcement phase of Fig. 1), the procedures changed to using a longer $(20 \mathrm{~s})$ time delay followed by graduated guidance, as well as differential reinforcement (where Sam was only given the opportunity to play if he 


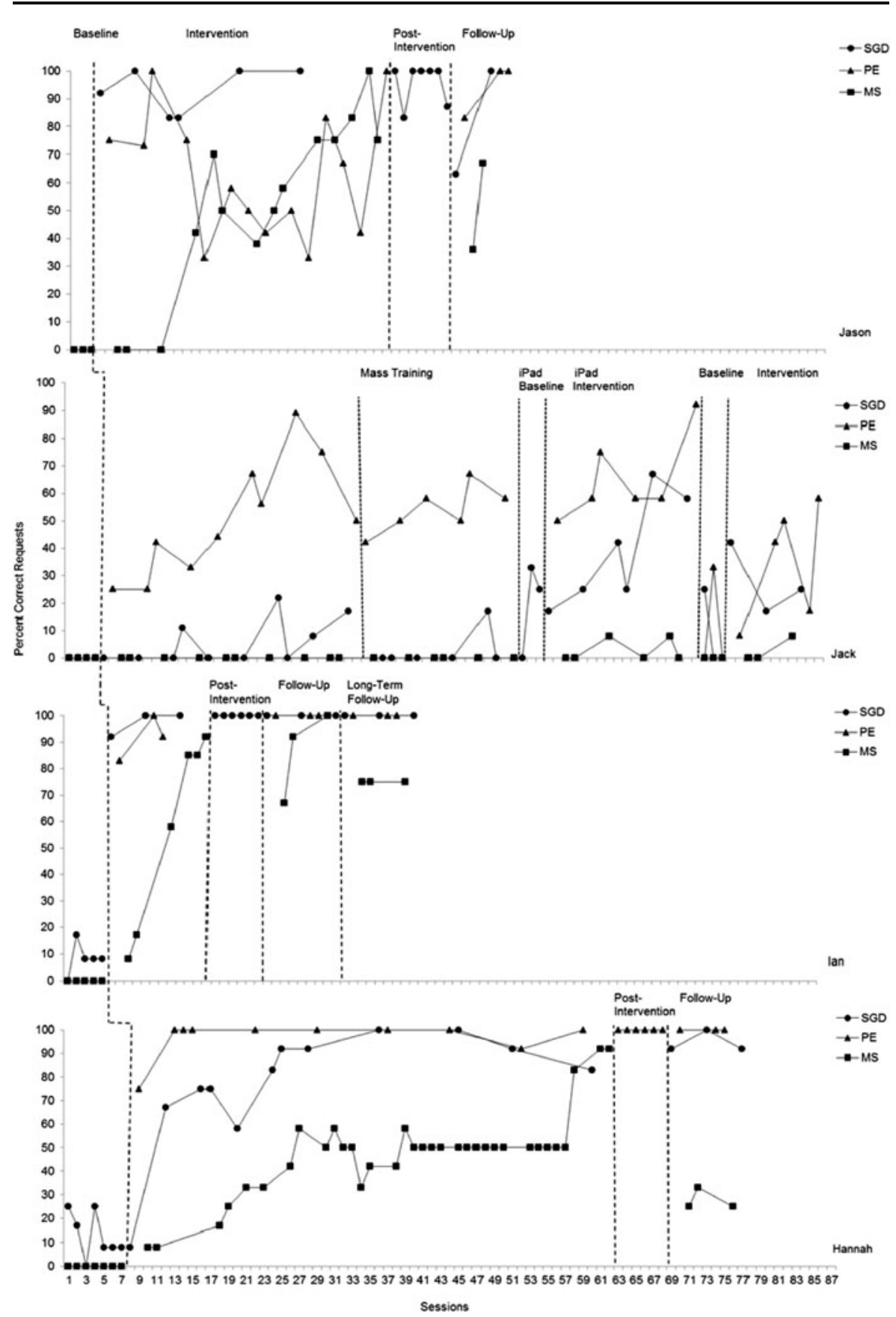

Fig. 1 Percentage of correct requests using the SGD, PE, and MS options across sessions for each participant

independently used the SGD or MS to request to play). Prompted trials were not reinforced. Because little progress was evident with these changes, a $0 \mathrm{~s}$ time delay was then implemented and immediate reinforcement was reintroduced (See the $0 \mathrm{~s}$ Time Delay and Differential Reinforcement phase of Fig. 1). That is, Sam was 
immediately prompted to make a correct request and then given access to the tray. However, for the MS option Sam appeared to become dependent on the trainer immediately prompting a correct response. He did not attempt to make the sign for PLAY and so for this reason, a $10 \mathrm{~s}$ time delay with reinforcement was reintroduced (10 s Time Delay and Differential Reinforcement phase of Fig. 1).

AAC Preference Assessments These assessments were undertaken to determine if participants would show a preference for using one of the three AAC options. They were undertaken after every sixth intervention session (i.e., after two sessions for each AAC option). During each preference assessment, the SGD, PE, and MS options were presented (randomly) at different positions on the table. While pointing to each option, the trainer asked the participant: Which communication option would you like to use? The SGD, PE, or MS? The child had $10 \mathrm{~s}$ in which to make a choice by touching one of the options. Once a choice was made, the trainer initiated one requesting opportunity with the chosen $\mathrm{AAC}$ option before reverting back to initiating requesting opportunities with the AAC device that was scheduled to be used for the session. If the child did not choose an option within $10 \mathrm{~s}$, the device preference assessment was terminated and training continued with the AAC option that was scheduled for use in that session.

Post-Intervention Once the participant reached criterion for each AAC device, postintervention preference assessments were introduced. These were identical to the previously described preference assessments, except that once an AAC option had been chosen, the participant continued to request preferred items using the chosen communication method for the entire 10-trial session.

Follow-up Six follow-up sessions were conducted 2 weeks following postintervention for Joe (Session 88 of Fig. 1) and Saskia (Session 85 of Fig. 1). Participants did not use either communication option during the break. Because Sam and Nicky did not complete all phases of the study due to time constraints, they did not receive any follow-up probes. Procedures for follow-up were identical to the intervention phase, except no prompting occurred and reinforcement was contingent upon a correct request. One AAC preference assessment was implemented before each follow-up session.

\section{Inter-Observer Agreement}

The trainer collected data on the frequency of correct requesting, the level of prompting required during intervention for each trial, as well as which communication mode was selected during the AAC preference assessments. To assess the reliability of the trainer's data collection, an independent observer also collected data on the frequency of requesting, level of prompting, and communication mode chosen. For each session, percentages of agreement between the independent observer and the trainer were calculated using the formula: Agreements/(Agreements + Disagreements) $\times 100 \%$. Inter-observer agreement data were collected on $28 \%$ of all sessions and ranged from 80 to $100 \%$ with a mean of $99.2 \%$. 


\section{Procedural Integrity}

To assess procedural integrity, the independent observer used a checklist of the procedures and recorded whether or not the trainer had correctly implemented each procedural step in its proper sequence. Procedural integrity was assessed on $28 \%$ of all sessions and ranged from 85 to $100 \%$ correct implementation of the procedural steps with an overall mean of $99.8 \%$. A second independent observer collected interobserver agreement data on $7 \%$ of these integrity checks with $100 \%$ agreement.

\section{Results}

Figure 1 shows the percentage of correct requests during each session/phase of the study and for each of the three AAC modes. Figure 2 provides a summary of the results from the AAC preference assessments conducted during intervention and subsequent phases. In baseline (Fig. 1), none of the participants ever used MS or $\mathrm{PE}$ to make the targeted requests. Saskia and Nicky made one and two correct SGDbased requests, respectively during baseline.

Joe When intervention was introduced, Joe reached the acquisition criterion for the MS option on his 15th MS training session. Similarly, when intervention was introduced, and then modified by removing the distractor symbols, Joe achieved acquisition with PE and SGD on his 16th and 17th intervention sessions, respectively. During the post-acquisition phase, Joe chose to use the SGD (55\%) more often than PE (45\%). Once chosen, he then used the selected option (i.e., either SGD or PE)

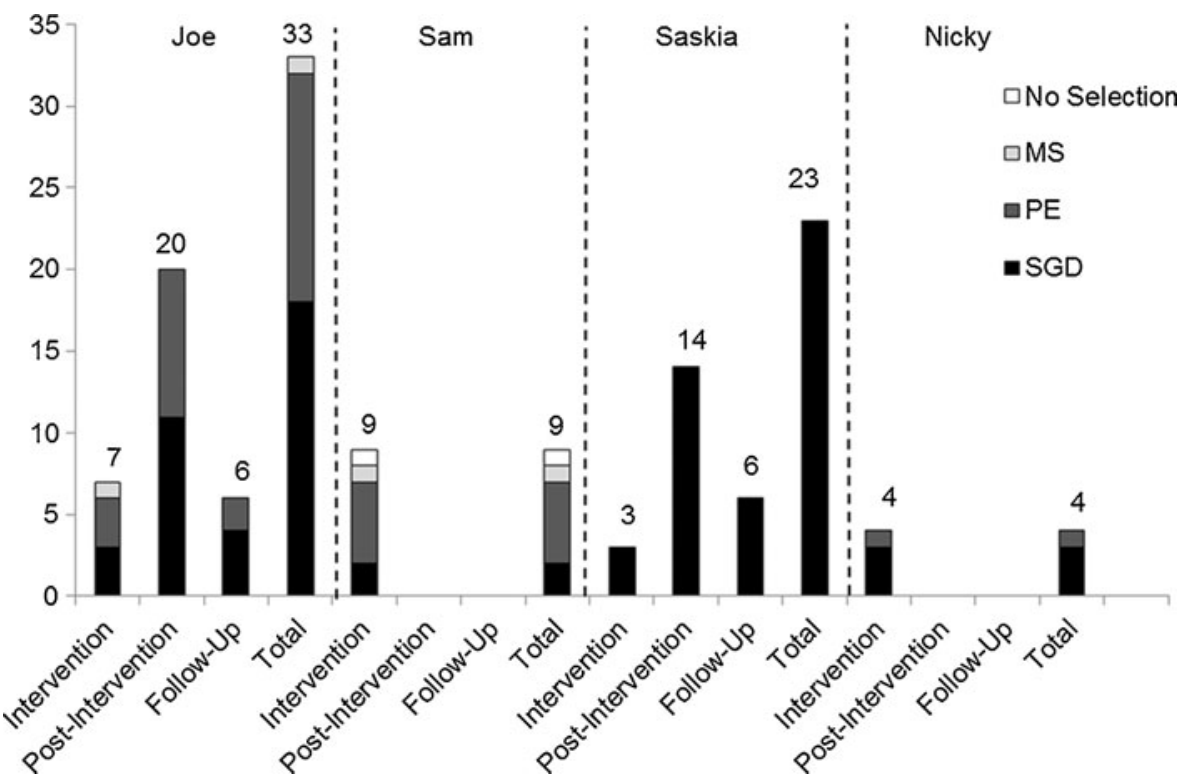

Fig. 2 Results from the device preference assessment probes depicting the number of times each communication option (SGD, PE, and MS) was chosen and the number of time a device was not chosen (No Selection) across each phase of the study for each participant 
with $100 \%$ proficiency. During follow-up, Joe maintained his level of correct SGDand PE-based requests at $100 \%$, but his performance dropped to 20 and $50 \%$ correct for MS. Overall, Joe received a total of 33 opportunities to choose between the SGD, PE and MS option (Fig. 2) and he chose the SGD most frequently (61\%).

Sam When intervention was introduced with Sam, he reached acquisition with PE on his 9th training session. When the intervention procedures were modified, he achieved acquisition of SGD on his 17th such session. However, even with additional procedural modifications, Sam did not achieve acquisition for MS within the timeframe of this study. Sam did not progress to the post-intervention or follow-up phases due to his failure to acquire use of the MS option. During intervention, Sam received nine AAC preference assessments (Fig. 2) and he chose PE most frequently (56\%).

Saskia Saskia achieved acquisition of PE-, MS- and SGD-based requests on her fifth, sixth, and eighth respective intervention sessions. During the post-intervention phase, Saskia always chose to use the SGD and then used it with 80 to $100 \%$ proficiency. During follow-up, her performance maintained at $100 \%$ correct for the SGD, but decreased to $40 \%$ and $0 \%$ correct for the PE and MS modes, respectively. Overall, Saskia received 23 AAC preference assessments (Fig. 2) during which she always chose the SGD.

Nicky Nicky achieved acquisition of SGD- and PE-based requests on her fifth and sixth respective intervention sessions. She showed an initial increase in the percentage of correct requests using MS, but failed to achieve acquisition within the timeframe of the study and did not progress to the post-intervention or follow-up phases. Across her four AAC preference assessments conducted during intervention (see Fig. 2), she chose the SGD three times (75\%).

\section{Discussion}

The present study extends previous research by van der Meer et al. (2012) by comparing acquisition of three common modes of AAC, namely SGD, PE, and MS. The findings suggest that the systematic instructional procedures used for each AAC option (Duker et al. 2004) were largely effective in teaching each participant to use at least two of the three AAC options. Furthermore, a key aspect of the study was to assess preferences for one mode of communication over the others throughout the intervention process, allowing participants some degree of self-determination with respect to AAC modes (Sigafoos 2006). Specifically, two participants (Joe and Saskia) reached criterion for use with each communication option and demonstrated a preference for using the SGD. The other two participants (Sam and Nicky) reached criterion for SGD and PE, but not MS. Nicky exhibited a preference for using the SGD, while Sam demonstrated a slight preference for using PE.

The findings support those of previous studies suggesting that students with DD can learn to use a SGD, PE, and MS for functional communication and that many will also indicate a preference for using a particular communication system (van der Meer 
et al. 2012, 2011b). The findings also provide further evidence indicating that most of the children assessed to date appear to show a preference for using SGD over PE and MS (van der Meer et al. 2012, 2011b), although the present study appears to be the only one to date that has compared acquisition of, and preference for SGD, PE, and MS.

While all of the participants learned to use PE and SGD, Sam and Nicky failed to reach criterion for MS, even with modifications to the intervention process. This finding could suggest that MS communication is more difficult for some children to learn or that the instructional procedures used in the present study were better suited for teaching use of the SGD and PE options. With respect to the first possibility, Iacono and colleagues (Iacono and Duncum 1995; Iacono et al. 1993) suggested that graphic symbols, such as those used for the SGD and PE options in this study, are less demanding on children's working memory because only recognition memory is needed, whereas MS requires the use of recall memory. This could be one reason why MS is sometimes learned at a slower rate than other AAC systems and this might also explain some of the patterns with respect to preferences for SGD and PE over MS.

Alternatively, MS might simply be a more difficult AAC system to teach because forming the signs requires more and varied physical movements than simply pointing to or handing a graphic to a partner (van der Meer et al. 2012). Another possibility is that Sam and Nicky's failure to reach criterion for MS reflected the fact that they did not prefer to use it and were therefore less motivated to participate in the MS intervention sessions, once they started to make progress with the other options. This possibility suggests that preference, or lack of preference, for an AAC option may influence motivation to learn to use that option. If this explanation has validity, it would highlight the value of assessing preferences for different AAC options during the early stages of intervention, as was attempted in the present study. However, it is unclear how early such assessments might be implemented. Pre-baseline assessments could be configured, for example, but it is unclear if participants would require some level of exposure to each option before their choices would represent valid indicators of preference.

For one participant (Joe), it appeared that discrimination of graphic symbols was difficult. Specifically, Joe did not learn to discriminate the SNACK symbol from the PLAY symbol, and only reached criterion for SGD and PE when the distracter (PLAY) symbol was removed. As with Sam and Nicky's failure to reach criterion for MS, Joe's difficulty could reflect either a problem in his discrimination learning abilities or ineffective instructional procedures. In any event, these problems in teaching Sam, Nicky, and Joe suggest there may be some value in implementing a pre-intervention assessment of children's learning and behavioral characteristics (Light et al. 1998), such as determining the level of iconicity appropriate for an individual to acquire graphic symbol and MS understanding (Koul et al. 2001). From a research perspective it is important to ensure each AAC system is comparable in terms of cognitive demands so as to maintain functional equivalence in order to compare acquisition and preference between AAC systems (Schlosser 2003b). It might also be important to ensure a match between the AAC system and skills being taught and the instructional strategies that are implemented to teach that system and those skills. 
A limitation of the present study is that the PE system was not equivalent to the SGD and MS systems. While the SGD and MS options required only a one-step request, the former included two steps. That is, for the PE option, participants were required to not only place the I WANT, but also the corresponding SNACK or PLAY symbols, depending on whether they were requesting snacks or toys, onto the two locations provided on a separate card. This may have increased the response effort for the PE system, which in turn may have negatively influenced acquisition and preferences (Ringdahl et al. 2009; Winborn-Kemmerer et al. 2009). Although this did not appear to influence acquisition of the PE system in the present study, it may have diminished preference for that system. However, Sam did in fact demonstrate a preference for PE. Furthermore, due to the inherent differences in response topographies of SGD and PE versus MS, another potential limitation outlined by van der Meer et al. (2012) was that a MS response could be produced during SGD and PE sessions, but not vice versa, possibly influencing preferences and rapidity of acquisition.

Joe, Saskia, and Nicky appeared to show a preference for using the SGD. While this could suggest that it was easier to use than either the PE or MS option, it is also possible that the SGD required somewhat more refined motor control, which might in fact make this a more difficult option to learn. That is, activating the speech-output function of the iPod-based SGD required a level of finesse (i.e., lightly touching or tapping the icon), which has been documented to be difficult for some adolescents with DD to master (Kagohara et al. 2010; van der Meer et al. 2011a, 2012). Despite what could be a slightly more difficult system to activate, Joe, Saskia, and Nicky showed a preference for using the SGD. While van der Meer et al. (2012) suggested that some participants may prefer AAC options that are easier to use, others may prefer SGD due to the dynamic display and speech-output features. Therefore, perhaps in addition to ease of use, it could be hypothesized that inherent features of some AAC options (e.g., speech-output) influence such preferences as suggested by Sigafoos et al. (2005). While our results suggest children showed idiosyncratic preferences for the AAC options, future research would be needed to determine variables that might influence such preferences.

Joe and Sam did not come to make any consistent choices for one communication device over the others until they had reached criteria with each system. Saskia and Nicky, in contrast, appeared to show a preference (for the SGD) before they had learned to use the communication options. These results suggest that preference for different AAC options may emerge at different times in the intervention process. In line with previous research (van der Meer et al. 2012) Joe and Saskia showed better performance during follow-up with their preferred communication option. This finding suggests that preference may influence maintenance of newly acquired AAC-based requesting skills. Future research is needed to examine whether these findings might extend to interventions that focus on teaching more complex communication skills, such as asking and answering questions and commenting on the environment.

It did not appear that differing reinforcement histories accounted for the children's preferences for the different AAC options because they received the same number of sessions/reinforcements with each option during baseline and intervention. While Sam did later receive differential reinforcement schedules for the SGD and MS 
options in an effort to increase his performance with these two options, he was already showing a preference for PE prior to this procedural manipulation. However, it could be that the children's prior (pre-baseline) experiences may have influenced their preferences to some degree. Specifically, prior to this study, it appeared that while none of the children had any experience with SGDs, they reportedly had experience with one or more of the other AAC modes. Joe, Sam, and Saskia, for example, were reported to have had prior experience with manual signing and Joe and Nicky were reported to have had some experience with picture-based communication systems. Unfortunately, it is impossible to know if any of these prior experiences influenced their learning rates and choices during the AAC preference assessments that were conducted in this study. Future research could be improved by controlling for the potential bias that may arise when children enter a study with differing amounts and types of prior experiences with the to be compared AAC options. In practice, however, it may be difficult to determine the precise amount and nature of any such prior experiences given the often subjective and anecdotal nature of the information available to researchers about children's prior AAC experiences. It is also perhaps inevitable that children with DD who have limited or no speech will be exposed to one or more AAC modes and that such exposure could influence acquisition of, and preference for, different AAC modes. We would argue that even with such difficulties and uncertainties with respect to children's prior AAC experiences, it would still seem useful to assess their preference for different AAC options so as to promote greater self-determination.

In summary, the results of present study extend the findings of van der Meer et al. (2012) by comparing acquisition of, and preference for, three commonly used AAC modes (SGD, PE, and MS) among four children with DD. The results showed that two children learned to use all three AAC modes, whereas the other two children learned to use SGD and PE, but not MS. Preference checks suggested that three of the four children appeared to prefer using the SGD, whereas the other child showed a preference for using PE. Preference appeared to influence acquisition and maintenance, but more research is needed to confirm any such effects.

Acknowledgments Support for this research was provided from the New Zealand Government through the Marsden Fund Council, administered by the Royal Society of New Zealand; and by Victoria University of Wellington, The University of Canterbury, and The New Zealand Institute of Language, Brain \& Behaviour.

Declaration of Interests The authors report no conflicts of interests. The authors alone are solely responsible for the content and writing of this paper.

\section{References}

Adkins, T., \& Axelrod, S. (2001). Topography-versus selection-based responding: comparison of mand acquisition in each modality. The Behavior Analyst Today, 2, 259-266.

Beck, A., Stoner, J., Bock, S., \& Parton, T. (2008). Comparison of PECS and the use of a VOCA: a replication. Education and Training in Developmental Disabilities, 43, 198-216.

Beukelman, D., \& Mirenda, P. (2005). Augmentative and alternative communication: Supporting children and adults with complex communication needs (3rd ed.). Baltimore: Paul H. Brookes Publishing Co. 
Bock, S., Stoner, J., Beck, A., Hanley, L., \& Prochnow, J. (2005). Increasing functional communication in non-speaking preschool children: comparison of PECS and VOCA. Education and Training in Developmental Disabilities, 40, 268-278.

Bondy, A., \& Frost, L. (1994). The picture exchange communication system. Focus on Autistic Behavior, 9 , $1-19$.

Bondy, A., \& Frost, L. (2001). The picture exchange communication system. Behavior Modification, 25, $725-744$.

Cannella-Malone, H., DeBar, R., \& Sigafoos, J. (2009). An examination of preference for augmentative and alternative communication devices with two boys with significant intellectual disabilities. Augmentative and Alternative Communication, 25, 262-273.

DeLeon, I., \& Iwata, B. (1996). Evaluation of a multiple-stimulus presentation format for assessing reinforcer preferences. Journal of Applied Behavior Analysis, 29, 519-532.

Duker, P., Didden, R., \& Sigafoos, J. (2004). One-to-one training: Instructional procedures for learners with developmental disabilities. Austin: Pro-Ed.

Flippin, M., Reszka, S., \& Watson, L. (2010). Effectiveness of the Picture Exchange Communication System (PECS) on communication and speech for children with autism spectrum disorders: a metaanalysis. American Journal of Speech - Language Pathology, 19, 178-195.

Goldstein, H. (2002). Communication intervention for children with autism: a review of treatment efficacy. Journal of Autism and Developmental Disorders, 32, 373-394.

Green, V., Sigafoos, J., Didden, R., O’Reilly, M., Lancioni, G., \& Ollington, N. (2008). Validity of a structured interview protocol for assessing children's preferences. In P. Grotewell \& Y. Burton (Eds.), Early childhood education: Issues and developments (pp. 87-103). New York: Nova.

Gregory, M., DeLeon, I., \& Richman, D. (2009). The influence of matching and motor-imitation abilities on rapid acquisition of manual signs and exchange-based communication responses. Journal of Applied Behavior Analysis, 42, 399-404.

Hart, S., \& Banda, D. (2010). Picture Exchange Communication System with Individuals with developmental disabilities: a meta-analysis of single subject studies. Remedial \& Special Education, 31, 476488.

Iacono, T., \& Duncum, J. (1995). Comparison of sign alone and in combination with an electronic communication device in early language intervention: case study. Augmentative and Alternative Communication, 11, 249-259.

Iacono, T., Mirenda, P., \& Beukelman, D. (1993). Comparison of unimodal and multimodal AAC techniques for children with intellectual disabilities. Augmentative and Alternative Communication, 9, 83-94.

Kagohara, D., van der Meer, L., Achmadi, D., Green, V. A., O’Reilly, M. F., Mulloy, A., et al. (2010). Behavioral intervention promotes successful use of an iPod-based communication device by an adolescent with autism. Clinical Case Studies, 9, 328-338.

Kennedy, C. (2005). Single-case designs for educational research. Boston: Pearson Education Inc.

Koul, R., Schlosser, R., \& Sancibrian, S. (2001). Effects of symbol, referent, and instructional variables on the acquisition of aided and unaided symbols by individuals with autism spectrum disorders. Focus on Autism \& Other Developmental Disabilities, 16, 162-169.

Lancioni, G., O’Reilly, M., Cuvo, A., Singh, N., Sigafoos, J., \& Didden, R. (2007). PECS and VOCAs to enable students with developmental disabilities to make requests: an overview of the literature. Research in Developmental Disabilities, 28, 468-488.

Light, J., Roberts, B., Dimarco, R., \& Greiner, N. (1998). Augmentative and alternative communication to support receptive and expressive communication for people with autism. Journal of Communication Disorders, 31, 153-180.

Lloyd, L., Fuller, D., \& Arvidson, H. (1997). Augmentative and alternative communication: A handbook of principles and practices. Boston: Allyn \& Bacon.

Mirenda, P. (2003). Toward functional augmentative and alternative communication for students with autism: manual signs, graphic symbols, and voice output communication aids. Language, Speech, \& Hearing Services in Schools, 34, 203-216.

Nederlands Gebarencentrum (2006). Gebarenwoordenboek voor kinderen 1. Biklin: Author.

Preston, D., \& Carter, M. (2009). A review of the efficacy of the Picture Exchange Communication System intervention. Journal of Autism and Developmental Disorders, 39, 1471-1486.

Pyramid Educational Products. (2009). PICS for PECS 2009. Newport: Author.

Ringdahl, J., Falcomata, T., Christensen, T., Bass-Ringdahl, S., Lentz, A., \& Dutt, A. (2009). Evaluation of a pre-treatment assessment to select mand topographies for functional communication training. $R e-$ search in Developmental Disabilities, 20, 330-341. 
Rispoli, M., Franco, J., van der Meer, L., Lang, R., \& Carmargo, S. (2010). The use of speech generating devices in communication interventions for individuals with developmental disabilities: a review of the literature. Developmental Neurorehabilitation, 13, 276-293.

Rotholz, D., Berkowitz, S., \& Burberry, J. (1989). Functionality of two modes of communication in the community by students with developmental disabilities: a comparison of signing and communication books. Journal of The Association for Persons with Severe Handicaps, 14, 227-233.

Schlosser, R. (2003a). The efficacy of augmentative and alternative communication: Toward evidencebased practice. San Diego: Academic.

Schlosser, R. (2003b). Single-subject designs. In R. Schlosser (Ed.), The efficacy of augmentative and alternative communication: Toward evidence-based practice (pp. 85-143). San Diego: Academic.

Schlosser, R., \& Sigafoos, J. (2006). Augmentative and alternative communication interventions for persons with developmental disabilities: narrative review of comparative single-subject experimental studies. Research in Developmental Disabilities, 27, 1-29.

Schlosser, R., \& Wendt, O. (2008a). Augmentative and alternative communication intervention for children with autism. In J. Luiselli, D. Russo, W. Christian, \& S. Wilczynski (Eds.), Effective practices for children with autism: Educational and behavioral support interventions that work (pp. 325-389). Oxford: Oxford University Press.

Schlosser, R., \& Wendt, O. (2008b). Effects of augmentative and alternative communication intervention on speech production in children with autism: a systematic review. American Journal of SpeechLanguage Pathology, 17, 212-230.

Sigafoos, J. (1998). Choice making and personal selection strategies. In J. Luiselli \& M. Cameron (Eds.), Antecedent control: Innovative approaches to behavioral support (pp. 187-221). Baltimore: Paul H. Brookes Publishing Co.

Sigafoos, J. (2006). Editorial: self-determination: can we let the child determine the "best" treatment? Pediatric Rehabilitation, 9, 1-2.

Sigafoos, J., Drasgow, E., \& Schlosser, R. (2003). Strategies for beginning communicators. In R. Schlosser (Ed.), The efficacy of augmentative and alternative communication: Toward evidence-based practice (pp. 323-346). San Diego: Academic.

Sigafoos, J., O’Reilly, M., Ganz, J., Lancioni, G., \& Schlosser, R. (2005). Supporting self-determination in AAC interventions by assessing preference for communication devices. Technology and Disability, 17, $1-11$.

Sigafoos, J., Green, V., Payne, D., Son, S., O’Reilly, M., \& Lancioni, G. (2009). A comparison of picture exchange and speech-generating deivces: acquisition, pereference, and effects on social interaction. Augmentative and Alternative Communication, 25, 99-109.

Son, S., Sigafoos, J., O’Reilly, M., \& Lancioni, G. (2006). Comparing two types of augmentative and alternative communication systems for children with autism. Pediatric Rehabilitation, 9, 389395 .

Soto, G., Belfiore, P., Schlosser, R., \& Haynes, C. (1993). Teaching specific requests: a comparative analysis of skill acquisition and preference using two augmentative and alternative communication aids. Education and Training in Mental Retardation, 28, 169-178.

Sparrow, S., Balla, D., \& Cicchetti, D. (2003). Vineland $-Z$ adaptive behavior scales (Dutch ed.). Leiden: PITS.

Sulzer-Azaroff, B., Hoffman, A., Horton, C., Bondy, A., \& Frost, L. (2009). The Picture Exchange Communication System (PECS): what do the data say? Focus on Autism \& Other Developmental Disabilities, 24, 89-103.

Tien, K. (2008). Effectiveness of the Picture Exchange Communication System as a functional communication intervention for individuals with autism spectrum disorders: a practice-based research synthesis. Education and Training in Developmental Disabilities, 43, 67-76.

Tincani, M. (2004). Comparing the Picture Exchange Communication System and sign language training for children with autism. Focus on Autism \& Other Developmental Disabilities, 19, 152-163.

van der Meer, L., \& Rispoli, M. (2010). Communication interventions involving speech-generating devices for children with autism: a review of the literature. Developmental Neurorehabilitation, 13, 294-306.

van der Meer, L., Kagohara, D., Achmadi, D., Green, V. A., Herrington, C., Sigafoos, J., et al. (2011a). Teaching functional use of an iPod-based speech-generating device to students with developmental disabilities. Journal of Special Education Technology, 26, 1-12.

van der Meer, L., Sigafoos, J., O’Reilly, M., \& Lancioni, G. (2011b). Assessing preferences for AAC options in communication interventions for individuals with developmental disabilities: a review of the literature. Research in Developmental Disabilities, 32, 1422-1431. 
van der Meer, L., Kagohara, D., Achmadi, D., O’Reilly, M., Lancioni, G., Sutherland, D., et al. (2012). Speech-generating devices versus manual signing for children with developmental disabilities. Research in Developmental Disabilities.

Wendt, O. (2009). Research on the use of manual signs and graphic symbols in autism spectrum disorders: A systematic review. In P. Mirenda \& T. Iacono (Eds.), Autism spectrum disorders and AAC (pp. 83140). Baltimore: Paul H. Brookes Publishing Co.

Winborn-Kemmerer, L., Ringdahl, J., Wacker, D., \& Kitsukawa, K. (2009). A demonstration of individual preference for novel mands during functional communication training. Journal of Applied Behavior Analysis, 42, 185-189. 


\section{APPENDIX G}

van der Meer, L., Sutherland, D., O'Reilly, M., Lancioni, G., \& Sigafoos, J. (2012). A further comparison of manual signing, picture exchange, and speech-generating devices as communication modes for children with autism spectrum disorders. Research in Autism Spectrum Disorders, 6, 1247-1257 
Provided for non-commercial research and education use. Not for reproduction, distribution or commercial use.

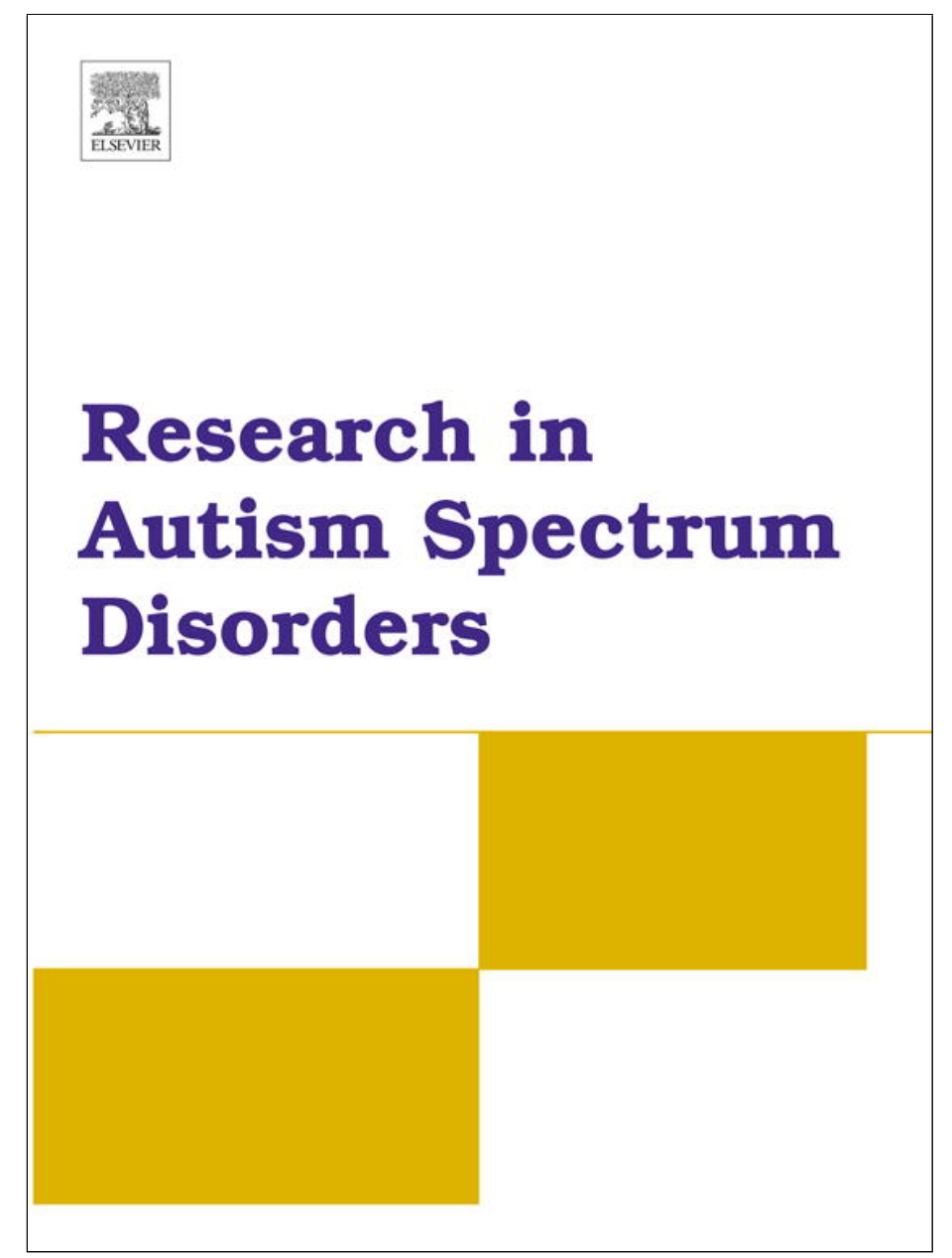

(This is a sample cover image for this issue. The actual cover is not yet available at this time.)

This article appeared in a journal published by Elsevier. The attached copy is furnished to the author for internal non-commercial research and education use, including for instruction at the authors institution and sharing with colleagues.

Other uses, including reproduction and distribution, or selling or licensing copies, or posting to personal, institutional or third party websites are prohibited.

In most cases authors are permitted to post their version of the article (e.g. in Word or Tex form) to their personal website or institutional repository. Authors requiring further information regarding Elsevier's archiving and manuscript policies are encouraged to visit:

http://www.elsevier.com/copyright 


\title{
A further comparison of manual signing, picture exchange, and speech-generating devices as communication modes for children with autism spectrum disorders
}

\author{
Larah van der Meer $^{\mathrm{a}, *}$, Dean Sutherland ${ }^{\mathrm{b}}$, Mark F. O’Reilly ${ }^{\mathrm{c}}$, Giulio E. Lancioni ${ }^{\mathrm{d}}$, Jeff Sigafoos ${ }^{\mathrm{a}}$ \\ ${ }^{a}$ Victoria University of Wellington, New Zealand \\ ${ }^{\mathrm{b}}$ University of Canterbury, New Zealand \\ ${ }^{\mathrm{c}}$ Meadows Center for Preventing Educational Risk, University of Texas at Austin, United States \\ ${ }^{\mathrm{d}}$ University of Bari, Italy
}

\section{A R T I C L E I N F O}

\section{Article history:}

Received 20 April 2012

Accepted 20 April 2012

\section{Keywords:}

Augmentative and alternative

communication

Autism spectrum disorders

Manual signing

Picture exchange communication

Preference assessment

Specific requesting

Speech-generating devices

\begin{abstract}
A B S T R A C T
We compared acquisition of, and preference for, manual signing (MS), picture exchange $(\mathrm{PE})$, and speech-generating devices (SGDs) in four children with autism spectrum disorders (ASD). Intervention was introduced across participants in a non-concurrent multiple-baseline design and acquisition of the three communication modes was compared in an alternating treatments design. Children's preference for using MS, PE or the SGD was also assessed. With intervention, all four participants learned to make specific requests using at least one of the three communication modes. The children also showed a preference for one mode. These results extend previous studies by demonstrating (in four new children with ASD) differential acquisition of, and idiosyncratic preferences for, three commonly used alternative communication modes. The present results further suggest faster acquisition and better maintenance with the preferred mode. We conclude that children's preferences for MS, PE, and SGDs should be considered when designing and implementing augmentative and alternative communication interventions.
\end{abstract}

(c) 2012 Elsevier Ltd. All rights reserved.

\section{Introduction}

Many children with autism spectrum disorder (ASD) fail to develop speech or have limited/unintelligible speech (Matson, Mahan, Kozlowski, \& Shoemaker, 2010). These individuals might learn to communicate using some form of augmentative and alternative communication (AAC; Beukelman \& Mirenda, 2005; Schlosser, 2003). Various AAC modes have been recommended for individuals with ASD, including manual signing (MS), picture exchange (PE; Bondy \& Frost, 2009), and electronic speech-generating devices (SGDs; Lancioni et al., 2007; Mirenda, 2003).

Systematic reviews of the literature provide empirical support for the use of each of these three AAC modes for individuals with ASD (Flippin, Reszka, \& Watson, 2010; van der Meer \& Rispoli, 2010; Wendt, 2009). However, the relative efficacy of these three AAC modes remains an open question (Mirenda, 2003; Schlosser \& Blischak, 2001). To address this issue, several studies have compared how quickly children have learned to use PE versus MS (Adkins \& Axelrod, 2001; Gregory, DeLeon, \& Richman, 2009; Tincani, 2004), PE versus SGDs (Beck, Stoner, Bock, \& Parton, 2008; Bock, Stoner, Beck, Hanley, \& Prochnow, 2005) and

\footnotetext{
* Corresponding author at: School of Educational Psychology, Victoria University of Wellington, PO Box 17-310, Karori 6147, Wellington, New Zealand. Tel.: +64 $44635233 \times 4597$.

E-mail address: larah.vandermeer@vuw.ac.nz (L. van der Meer).
} 
SGDs versus MS (Iacono \& Duncum, 1995; Iacono, Mirenda, \& Beukelman, 1993; Sigafoos \& Drasgow, 2001). As noted by van der Meer, Sigafoos, O'Reilly, and Lancioni (2011), the results of these comparisons have been somewhat equivocal. Consequently, these authors suggested that a child's preference for using one AAC mode over others may be an important variable to consider when designing and implementing AAC intervention.

Along these lines, two recent studies (van der Meer, Didden, et al., 2012; van der Meer, Kagohara, et al., 2012) compared not only acquisition, but also children's preferences for using different AAC modes. Specifically, van der Meer, Kagohara, et al., 2012 compared acquisition of, and preference for using, a SGD versus MS. The study focused on teaching a general request for preferred snacks or toys (e.g., "I want to play.") to four children (aged 5.5-10 years) with developmental disabilities. Intervention-consisting of offering preferred items, waiting $10 \mathrm{~s}$ for a correct request, and prompting and reinforcing correct requests-was introduced simultaneously with the MS and SGD mode in accordance with a multiplebaseline across participants and alternating treatments design. Choice-making opportunities, involving offering the children both AAC options and letting them choose one to use, occurred during and after intervention to determine if the children showed a preference for the SGD or MS mode. With intervention, all four children achieved acquisition of the SGD and three of the four also learned to use MS to make the general request. Interestingly, three children most often chose to use the SGD and showed better performance with the SGD, suggesting that preference might influence acquisition and maintenance or that acquisition might influence preference.

In the second study, van der Meer, Didden, et al. (2012) extended this comparison to three AAC modes, specifically SGD, $\mathrm{PE}$, and MS. This study involved four new children with developmental disabilities aged 6-13 years. The children were taught to make a general request for preferred snacks or toys using a SGD, PE, and MS. As with van der Meer, Kagohara, et al. (2012), the children learned to request, but at differing rates and to differing degrees of proficiency with the three modes. Specifically, while all four children reached the acquisition criteria with PE and the SGD, only two also achieved the acquisition criterion during MS instruction. Also, three of the four children showed a preference for the SGD, while the fourth preferred PE. These AAC preference assessment results were similar to those obtained by van der Meer, Kagohara, et al., 2012 Furthermore, the children's AAC preferences appeared to influence the rate of acquisition and performance during follow-up sessions. Specifically, children learned to use the more preferred AAC option quicker and showed better maintenance with the more preferred AAC option during follow-up.

Interesting questions arising from these two studies include (a) whether similar results would be found with additional children with ASD, (b) whether a child's relative preference for different AAC modes are present prior to intervention and remain stable over time after intervention, and (c) whether the findings of van der Meer, Didden, et al. (2012) and van der Meer, Kagohara, et al. (2012) would have generality when teaching more specific requesting forms (e.g., "I want to play with the ball." "I want to play with the doll.") as compared to the more general requesting forms (e.g., "I want to play.") taught in the two previous studies of van der Meer, Didden, et al., 2012 and van der Meer, Kagohra, et al., 2012 A final question is whether parents and general teaching staff could learn to effectively implement the intervention procedures successfully applied by research staff in our two previous studies (i.e., van der Meer, Didden, et al., 2012 and van der Meer, Kagohara, et al., 2012).

The purpose of the current study was therefore to assess: (a) whether four new children with ASD diagnoses could be taught by their parents and general teaching staff to use specific requesting forms with three different AAC modes (i.e., SGD, PE, and MS); (b) whether these participants would demonstrate a preference for one communication modality over the others prior to and during intervention; (c) whether any such preferences would be stable over time; and (d) whether any such preferences would influence acquisition rates and subsequent performance during follow-up sessions. Based on the previous research reviewed above, we hypothesized that the four participants would learn to make specific requests at varying rates with the three communication modes, but that they would make the slowest progress with MS. We further hypothesized that most children would prefer using the SGD and that their relative preference for the three AAC modes would be stable over time. Finally, we predicted that the children would show better maintenance of requesting with their most preferred AAC mode.

\section{Method}

\subsection{Ethical clearance and informed consent}

The relevant university ethics committee approved the study. Consent was obtained for the children to participate from their parents. Children's assent was inferred from their general compliance throughout each session.

\subsection{Participants}

Four children who met the following inclusion criteria participated in this study: (a) diagnosis of ASD, (b) school-aged children of less than 18 years of age, (c) very limited or no communication skills as determined by an age equivalency of 2.5 years or less in the Expressive Communication Sub-Domain of the Vineland Adaptive Behavior Scales (Sparrow, Cicchetti, \& Balla, 2005), (d) no auditory or visual impairments that would interfere with the use of AAC, and (e) sufficient motor skills to operate each of the three AAC communication modes, as determined by an age equivalency of 1.0 year or more on the Fine Motor Skills Sub-Domain of the Vineland-II. 


\subsubsection{Jason}

Jason was a 4-year-old male diagnosed with autism and global developmental delay. On the communication sub-domain of the Vineland-II (Sparrow et al., 2005), Jason received age equivalencies of 2:5 (years:months) for receptive communication, 1:8 for expressive communication, and 3:1 for written communication. His age equivalency on the fine motor skills sub-domain of the Vineland-II was 2:11. Jason was reportedly able to use a few single words to make requests and comment on his environment, although his speech was largely unintelligible. He also appeared to attempt to communicate his wants and needs by taking people's hands and leading them. Jason had been informally introduced to PE in the past, but was not receiving any such training throughout the period of this study. This prior PE experience was independent of, and unrelated to, the current intervention. He did not have any prior experience with SGD or MS to request preferred objects.

\subsubsection{Jack}

Jack was a 4-year-old male diagnosed with autism. His age equivalencies were 1:3, 0:8, and 1:10 on the receptive, expressive, and written communication sub-domains of the Vineland-II (Sparrow et al., 2005). Jack received an age equivalency of 2:0 on the Vineland-II for fine motor skills. He did not have any spoken language, but had prior experience with PE that was independent of, and unrelated to, the current intervention. As a result of this prior experience, he was reportedly able to discriminate among $20 \mathrm{PE}$ symbols that he used to request food items. Otherwise his communication attempts involved leading a person's hand to desired objects. He had no experience with SGD or MS for requesting preferred items.

\subsubsection{Ian}

Ian was a 10-year-old male diagnosed with autism, moderate intellectual disability, developmental co-ordination disorder, and epilepsy. He received age equivalencies of 2:2, 1:4, and 3:10 on the receptive, expressive, and written communication sub-domains of the Vineland-II (Sparrow et al., 2005). He received an age equivalency of 2:1 on the fine motor skills sub-domain of the Vineland-II. Ian had no formal means of expressing himself, but attempted to create his own signs for words, that were difficult to decipher. Ian also tried to sound out some words, but this was unintelligible. He would often take people's hands to direct them to what he wanted and frequently grabbed people in an apparent attempt to get their attention. Ian did not have any prior training with SGD, PE, or MS to request preferred items. Ian's mother indicated that he engaged in obsessive behavior (e.g., fixation on certain toys) and had difficulty with changes to routine.

\subsubsection{Hannah}

Hannah was an 11-year-old female diagnosed with autism, severe global developmental delay, and intellectual disability. On the Vineland-II (Sparrow et al., 2005), she received age equivalencies of 1:3 for receptive communication, 0:9 for expressive communication, and 2:5 for written communication. She received an age equivalency of 2:11 on the fine motor skills sub-domain of the Vineland-II. Hannah appeared to communicate her wants and needs by taking people's hands and leading them to objects. She had received training with PE and had been exposed to several gestures, as well as a BIGmack ${ }^{\circledR}$ SGD on several occasions. These prior experiences were independent of, and unrelated to, the present study. She appeared to make vocalizations to communicate pleasure and distress.

\subsection{Setting, intervention context, and trainer instruction}

Jason, Jack, and Ian received intervention in the dining room of their family home. Hannah received intervention sessions in a special education classroom that was part of a public primary school. For Jason, Jack, and Ian the procedures associated with this study were conducted at a table and implemented in a one-to-one format, consisting of the trainer (mother) and child. Hannah's intervention was implemented at a table in the special education classroom in a one-to-one context consisting of the trainer (teaching assistant) and Hannah.

Jack, Jason, and Ian's mothers were taught how to implement the procedures by the first author. This parent instruction involved (a) explaining the general aims, goals, and procedures of the study, (b) providing parents with step-by-step written instructions for each phase of the study and explaining these steps prior to each phase of the study, (c) modeling implementation of the steps during the first trials at the beginning of each new phase and then having the parent implement the remaining trials for that session, and (d) providing feedback at least once per week throughout each phase of the study. The same strategies were used to teach the teaching assistant how to implement the procedures with Hannah.

\subsection{Preferred stimuli}

Snacks and/or toys that the participants seemed to prefer, and which would be appropriate for the participants to request during the snack/leisure activity, were identified using a two-stage stimulus preference assessment (Green et al., 2008). Stage 1 involved an indirect assessment in which parents/teachers were asked to list snacks and toys that the participants appeared to enjoy and would be appropriate for the intervention. The five to six most preferred stimuli were then selected for a direct stimulus assessment, involving the simultaneous presentation of multiple items, without replacement (DeLeon \& Iwata, 1996; Duker, Didden, \& Sigafoos, 2004). Each participant was presented with an array of five to six items from Stage 1 
(random placement) and allowed to select one. A session consisted of repeating such offers five or six times depending on whether five or six items had been placed on the tray. Items were not replaced once they had been selected. Toy and food items were assessed separately over six sessions (i.e., six sessions with toys and six sessions with foods). The most preferred foods and/or toys were identified by calculating a rank order of the percentage of times that an item was selected using the formula: number of selections/number of offers $\times 100 \%$.

Preferred stimuli selected for Jason were marshmallows (75\%), balloons and bubbles (both 67\%), and chips (35\%). Preferred stimuli for Jack were wafer biscuits (55\%), K'NEX ${ }^{\circledR}$ building blocks (similar to Lego ${ }^{\circledR} ; 43 \%$ ), waterplay toy (40\%), and caramel lollies (38\%). Preferred stimuli for Ian were music box (100\%), puzzles (40\%), hide and seek game (30\%), and bouncy balls (29\%). Preferred stimuli for Hannah were $M \& M^{\circledR}$ candy (50\%), bubbles (46\%), chocolate (43\%), and music box (32\%).

\subsection{Speech-generating device}

Jason, Jack, and Ian were taught to request preferred stimuli using an Apple iPod Touch ${ }^{\circledR}$ with Proloquo2Go ${ }^{\mathrm{TM}}$ software (Sennott \& Bowker, 2009). The iPod Touch ${ }^{\circledR}$ was placed inside an iMainGo2 ${ }^{\circledR}$ speaker case to increase sound amplification. Hannah, and later Jack, were taught to use an Apple iPad ${ }^{\circledR}$ with Proloquo2Go ${ }^{\mathrm{TM}}$ software. The iPod Touch ${ }^{\mathbb{R}}$ and iPad ${ }^{\mathbb{R}}$ were configured to show a single page containing four graphic symbols $\left(2.5 \times 2.5 \mathrm{~cm}\right.$ for the iPod ${ }^{\circledR}$ and $9.5 \times 6 \mathrm{~cm}$ for the iPad $\left.{ }^{\circledR}\right)$, representing requests for specific snacks and toys (e.g., CHOCOLATE, BALL). The graphic symbols were photos of the items uploaded into the Proloquo2 $\mathrm{Go}^{\mathrm{TM}}$ software package. Touching each symbol activated corresponding synthetic speechoutput (e.g., "I would like some chocolate." and "I would like to play with my ball.").

\subsection{Picture exchange}

The PE system consisted of four laminated photos $(7 \mathrm{~cm} \times 7 \mathrm{~cm})$ of the participant's most preferred items, with the corresponding printed word written below the photo. Photos were affixed with Velcro ${ }^{\mathrm{TM}}$ to a laminated card $(22 \mathrm{~cm} \times 22 \mathrm{~cm})$. The pictures were randomly allocated to the four locations of the card.

\subsection{Manual signing}

Participants were also taught to request preferred stimuli using signs from the Makaton Sign Language System (Makaton New Zealand/Aotearoa, 1998-1999). Manual signing was represented by a laminated picture $(22 \mathrm{~cm} \times 22 \mathrm{~cm})$ with four line drawings $(8 \mathrm{~cm} \times 8 \mathrm{~cm})$ of the hand formations needed to make the sign for each of the children's preferred stimuli targeted for intervention.

\subsection{Response definitions and measurement}

For SGD use, correct responding was defined as independently (without a gestural or verbal prompt) touching the symbol on the screen of the SGD to activate the corresponding speech output in exchange for the item offered by the trainer. For PE, participants were required to independently (without gestural or verbal prompt) remove the corresponding picture from the PE card and hand it over to the trainer in exchange for the item being offered. Manual signing was defined as independent (without a gestural or verbal prompt) performance of the hand gestures to produce correct signs in exchange for the item offered by the trainer. The frequency of correct responding was calculated for each session. Sessions consisted of 12 trials (i.e., each of the four preferred items was offered three times). The order of offering items was counterbalanced across sessions.

\subsection{Experimental design}

An alternating treatments design was used to compare intervention performance across the SGD, PE, and MS options (Kennedy, 2005). The study phases (i.e., baseline, intervention, preference assessments, post-intervention, and follow-up) were implemented according to a non-concurrent multiple-baseline across participants design (Watson \& Workman, 1981). The participants received differing lengths of baseline, but started their baselines in a staggered fashion due to their being recruited into the study at different points in time. Training was first provided to Jason, then Jack, then Ian, and finally Hannah. Jack received additional phases following his initial intervention due to lack of progress. Specifically, he received a modified intervention that involved massed-practice trials with the SGD and MS options. After this, he received a baseline with a new SGD (e.g., an iPad ${ }^{\circledR}$ ), followed by an intervention to teach him to use this new SGD along with PE and MS. He then received a new preference assessment to identify additional items for him to request and a final intervention phase to teach him to request these new items.

\subsection{Session schedule}

Requesting sessions were conducted three to five days per week. The AAC option available (i.e., SGD, PE, or MS) was counterbalanced across sessions to prevent order effects (Kennedy, 2005). For all sessions, the participant and trainer were 
seated next to each other at a table. One or two additional observers/data collectors were seated nearby on some occasions to collect interobserver agreement data and conduct procedural integrity checks.

When a participant reached criterion for one AAC device (i.e., 80\% correct requesting across three consecutive sessions), teaching for that system was put on hold while it continued with the other communication systems, if necessary, until criterion was reached on the other systems. One maintenance session with the acquired AAC system was conducted after every third teaching session with the other (yet to be acquired) AAC systems.

\subsection{Procedures}

Participants were taught to request specific snacks or toys. To ensure symbol discrimination, participants had to select the graphic symbol from the SGD or PE board or produce the manual sign corresponding to the item being offered by the trainer. If a participant requested a different item to the one being offered, the trainer responded by saying: We are requesting (name of item) and pointed to the correct symbol on the SGD/PE board or modeled the correct sign. It was considered more natural to provide them with some feedback when they activated these (non-target) symbols or made the non-target sign, but counter-productive to discrimination training to consequate these non-target responses with access to preferred items. Similarly, producing a manual sign to request items during SGD or PE sessions and vice versa was ignored so as to bring the use of each device under stimulus control.

\subsubsection{Pre-baseline AAC preference assessments}

These assessments were undertaken to familiarize participants with each of the three communication modes (SGD, PE, and MS) and to determine if participants showed a preference for one mode over the other two before learning to use them. During these sessions, the SGD, PE, and MS options were placed on the table in randomly determined locations. The trainer briefly explained and demonstrated each communication mode (e.g., This is a PE board. You use it like this.). The trainer then asked the participant: Which communication option would you like to use? Participants were allowed $10 \mathrm{~s}$ to select one of the communication options. Selecting was defined as touching, holding, and/or manipulating the device. If the child did not select one of the communication options within $10 \mathrm{~s}$, the next trial was initiated by again asking the participant: Which communication option would you like to use? Communication options were not replaced once selected. Instead, the remaining communication options were then offered. This procedure was repeated across six sessions (three offers per session) and the percentage of times that each device was selected was calculated using the formula: number of selections/number of offers $\times 100 \%$. Pre-baseline preference assessments were not undertaken with Hannah due to an oversight.

\subsubsection{Baseline}

During this phase, a tray containing one of the participants' four preferred items was placed on the table in view, but out of reach. The SGD, PE, and MS communication options were randomly placed on the table. Each session began with the trainer asking the participant to Let me know if you want this, while holding the tray with the item being offered. After $10 \mathrm{~s}$, the trainer moved the tray within reach and allowed the participant to take the item. This was repeated 12 times (trials) per session, with each of the four items offered three times in a counterbalanced order. This 10-s fixed-time schedule of reinforcement was provided to ensure continued motivation to participate in sessions. Participants were given approximately $20 \mathrm{~s}$ to consume/play with the item being offered. Responses to the SGD, PE, and MS were recorded, but had no programmed consequences.

\subsubsection{Intervention}

This phase was conducted in a discrete-trial format until participants reached criterion (i.e., $80 \%$ correct requesting across three consecutive sessions for each AAC mode) or, failing that, until they had received at least 10 sessions of intervention with each AAC mode. Each trial consisted of the trainer placing a tray with one of the participant's preferred items on the table, showing the item to the participant, but keeping it out of reach, and saying, Let me know if you want this. Training involved a 10-s time delay between the verbal cue (i.e., Let me know if you want this.) and the use of graduated guidance to prompt a correct request. Graduated guidance involved using the least amount of physical guidance necessary to prompt the child to make a request, with a simultaneous explanation of how to perform the action (e.g., Press the ball icon to ask to play with a ball. for SGD, or Hand over the picture of the ball. for PE, or Move your hands in the formation of a ball to make the sign for ball. for MS). Immediately after a correct SGD, PE, or MS request, the trainer moved the tray containing that item within reach of the participant with simultaneous social reinforcement (e.g., Good asking.). The participant was allowed approximately $20 \mathrm{~s}$ to consume/play with that item. After this, the next trial was initiated. Twelve such trials (three trials per item) were conducted per session with the order of the items being offered counterbalanced to avoid order effects.

\subsubsection{Procedural modifications}

Because Jack made little progress with the SGD and MS options during the initial intervention sessions, his teaching procedures were modified to include 10 massed-practice trials before each SGD and MS session. This involved implementing 10 consecutive graduated guidance trials with no time delay and no reinforcement. The aim was to provide Jack with additional practice in making the target responses. With this modification, Jack began to initiate correct requests with the SGD, but appeared to struggle to press the icons with the appropriate degree of finesse to activate the voice output. It was 
therefore decided to switch to an $\mathrm{iPad}^{\mathbb{R}}$ because we thought that the larger size of icons and increased sensitivity of the screen on the iPad ${ }^{\circledR}$ would make it easier for Jack to activate the SGD. Although Jack made some progress with these modifications to the intervention process, he also rather quickly appeared to lose interest in the preferred stimuli that he was being taught to request. Therefore another stimulus preference assessment was implemented. New preferred stimuli were then chosen for intervention consisting of lollies (60\%), blocks (46\%), an alphabet toy (40\%), and chips (24\%).

\subsubsection{AAC preference assessments}

These assessments were undertaken after each baseline, intervention, and follow-up session to determine if participants would show a preference for using one of the three AAC systems. They were identical to pre-baseline device preference assessments, except when the participant selected a communication mode, the trainer then initiated one requesting opportunity with the chosen mode before reverting back to another baseline, intervention, or follow-up session. If the participant did not choose an option within $10 \mathrm{~s}$, that AAC preference assessment trial was terminated and training continued with the AAC option that was scheduled for use in that session.

\subsubsection{Post-intervention}

Once the participant reached criterion for each AAC device, post-intervention preference assessments were introduced. These were identical to the previously described AAC preference assessments, except that once an AAC option had been chosen, the participant continued to request preferred items using the chosen communication method for the entire session.

\subsubsection{Follow-up}

Nine follow-up sessions (three for each communication mode) were conducted after Session 24 for Ian and after Session 45 for Jason (see Fig. 1). These occurred three weeks after their last post-intervention session. Ian also received a second set of [long-term] follow-up sessions beginning eight months after his previous follow-up sessions (Session 33 of Fig. 1). Hannah received follow-up after Session 68 (Fig. 1), eight weeks after her last post-intervention session. Because of a change of schools in the interim, Hannah's follow-up sessions were conducted by the first author, not by the teaching assistant. During the interval, participants did not use any of the communication modes to request their preferred items. Jack did not receive follow-up because he did not reach criterion for any of the communication modes. Procedures for follow-up were identical to the intervention phase, except no prompting occurred and participants only received access to preferred items contingent on correct requesting. Also an AAC preference assessment (as described in Section 2.11.5) was implemented after each session.

\subsection{Interobserver agreement}

Trainers collected data on the frequency of correct requesting and on which communication mode was selected during AAC preference assessments. To assess the reliability of the trainers' data collection, an independent observer also collected data on the frequency of requesting and communication mode chosen. For each session, percentages of agreement between the independent observer and the trainer were calculated using the formula: agreements/(agreements + disagreements $) \times 100$. These agreement checks occurred on 32\% of all sessions and ranged from 95 to $99.7 \%$ with a mean of $97.8 \%$.

\subsection{Procedural integrity}

To assess procedural integrity, the independent observer had a checklist of the procedural steps and recorded whether or not the trainer had correctly implemented each procedural step in its proper sequence. Procedural integrity was assessed on $32 \%$ of all sessions and ranged from 98.8 to $99.9 \%$ correct implementation with a mean of $99.1 \%$. A second independent observer collected inter-observer agreement data on $11 \%$ of these integrity checks with $100 \%$ agreement.

\section{Results}

Fig. 1 shows the percentage of correct requests during each session for each of the three AAC modes. Table 1 provides a summary of the pre-baseline preference assessments. Fig. 2 provides a summary of the results from the AAC preference assessments conducted during baseline, intervention, and subsequent phases. In baseline (Fig. 1), none of the participants ever used PE or MS to make the targeted requests. Ian and Hannah made several correct SGD-based requests during baseline. However, a stable and low baseline was evident for all children prior to introducing intervention.

\subsection{Jason}

When intervention was introduced, Jason reached the acquisition criterion for the SGD option on his third SGD training session (Fig. 1). Although he did not reach criterion for PE and MS, Jason achieved above $80 \%$ correct requesting in three of his PE $(M=86 \%$, range $=33-100 \%)$ and two of his $M S(M=51 \%$, range $=0-100 \%)$ intervention sessions. During the postintervention phase, Jason chose to use the SGD 100\% of the time. Once chosen, he then used the SGD with at least 80\% proficiency. During follow-up, Jason maintained correct SGD- and PE-based requests at high levels (63-100\%), but his performance dropped to between 36 and 67\% correct for MS. He chose the SGD first on 100\% of opportunities during the 


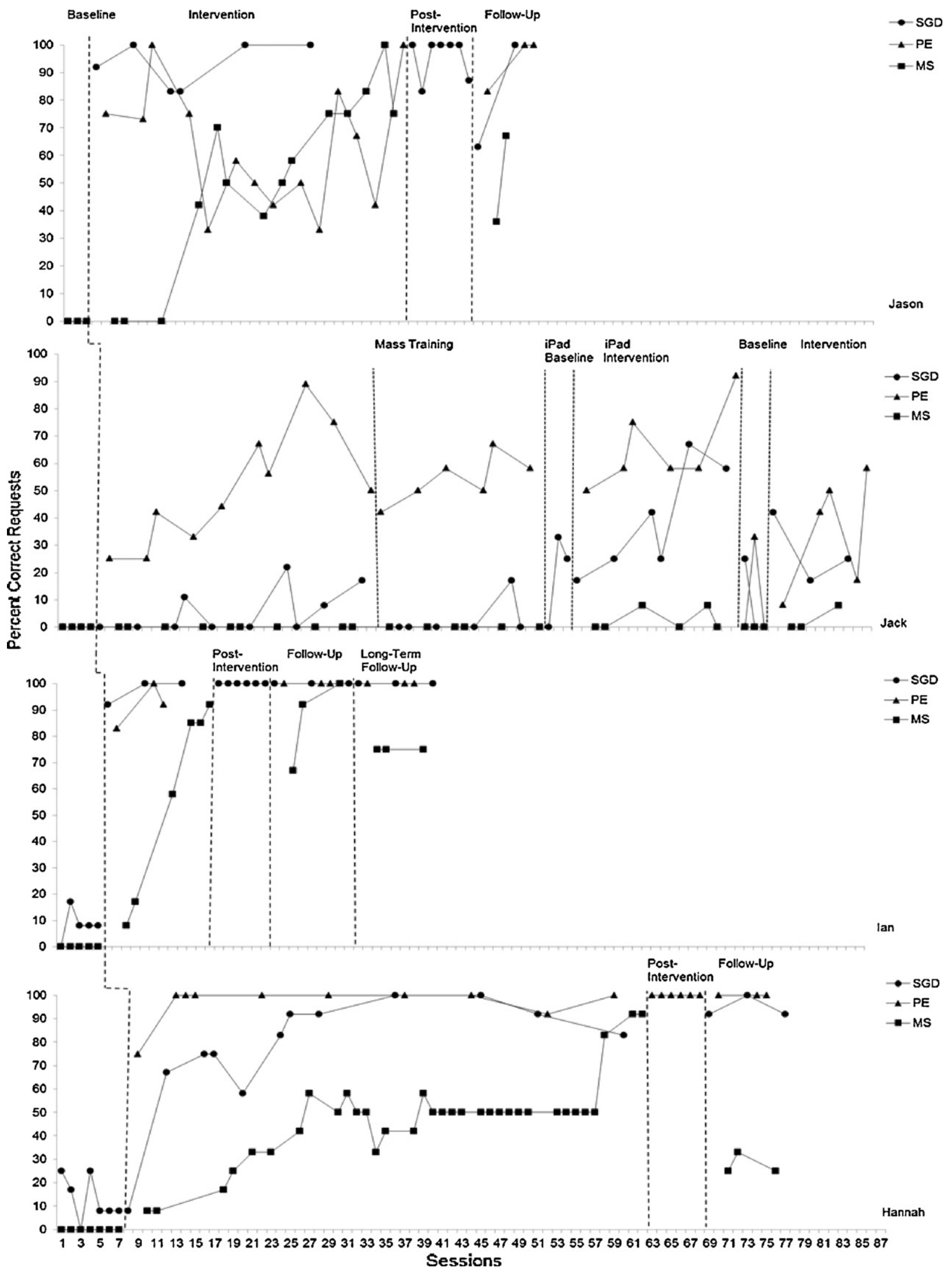

Fig. 1. Percentage of correct requests with SGD, PE, and MS across sessions for each participant. 
Table 1

Percentage of selection/times offered for SGD, PE, and MS during the pre-baseline AAC preference assessments.

\begin{tabular}{lrrr}
\hline Child & SGD & PE & MS \\
\hline Jason & 100 & 20 & 45 \\
Jack & 55 & 50 & 29 \\
Ian & 86 & - & 29 \\
Hannah & - & - \\
\hline
\end{tabular}

pre-baseline device preference assessments (Table 1). Overall, during baseline, intervention, post-intervention, and followup phases, Jason received a total of 45 opportunities to choose between the SGD, PE, and MS options (Fig. 2) and he chose the SGD most frequently (69\%).

\subsection{Jack}

Although Jack did not reach criterion with PE, he did achieve over 80\% correct requesting on a number of occasions. His performance with the PE mode averaged 52\% (range $=8-92 \%$ ) correct requesting. Similarly, even with modifications to the intervention procedures, he did not reach criterion with the iPod $\operatorname{Touch}^{\circledR}(M=5 \%$ range $=0-22 \%)$. When the iPad ${ }^{\circledR}$ was introduced, his performance increased to an average of $35 \%$ (range $=17-67 \%$ ) correct requesting. Even with additional procedural modifications, Jack made little progress with MS and did not achieve criterion within the timeframe of this study. Therefore he did not progress to the post-intervention or follow-up phases. Results from the pre-baseline preference assessment indicated that Jack chose each communication system comparably often (Table 1). During baseline and intervention, Jack received 89 AAC preference assessments (Fig. 2) and he chose PE most frequently (58\%), followed by the SGD (30\%).

\subsection{Ian}

Ian achieved criterion for SGD- and PE-based requesting on his third SGD and third PE intervention sessions (Fig. 1). He achieved criterion for MS-based requesting on his sixth MS intervention session (Fig. 1). During the post-intervention phase, Ian always chose to use the SGD and then used it with 100\% proficiency. During the first follow-up, his performance maintained at 100\% correct for the SGD and PE, but decreased with the MS option. During the second, long-term follow-up (after eight months), his performance with the SGD and PE option was at 100\%, whereas it was at 75\% for the MS option. Ian chose the SGD most frequently (86\%) during the pre-baseline AAC preference assessments (Table 1). During baseline,

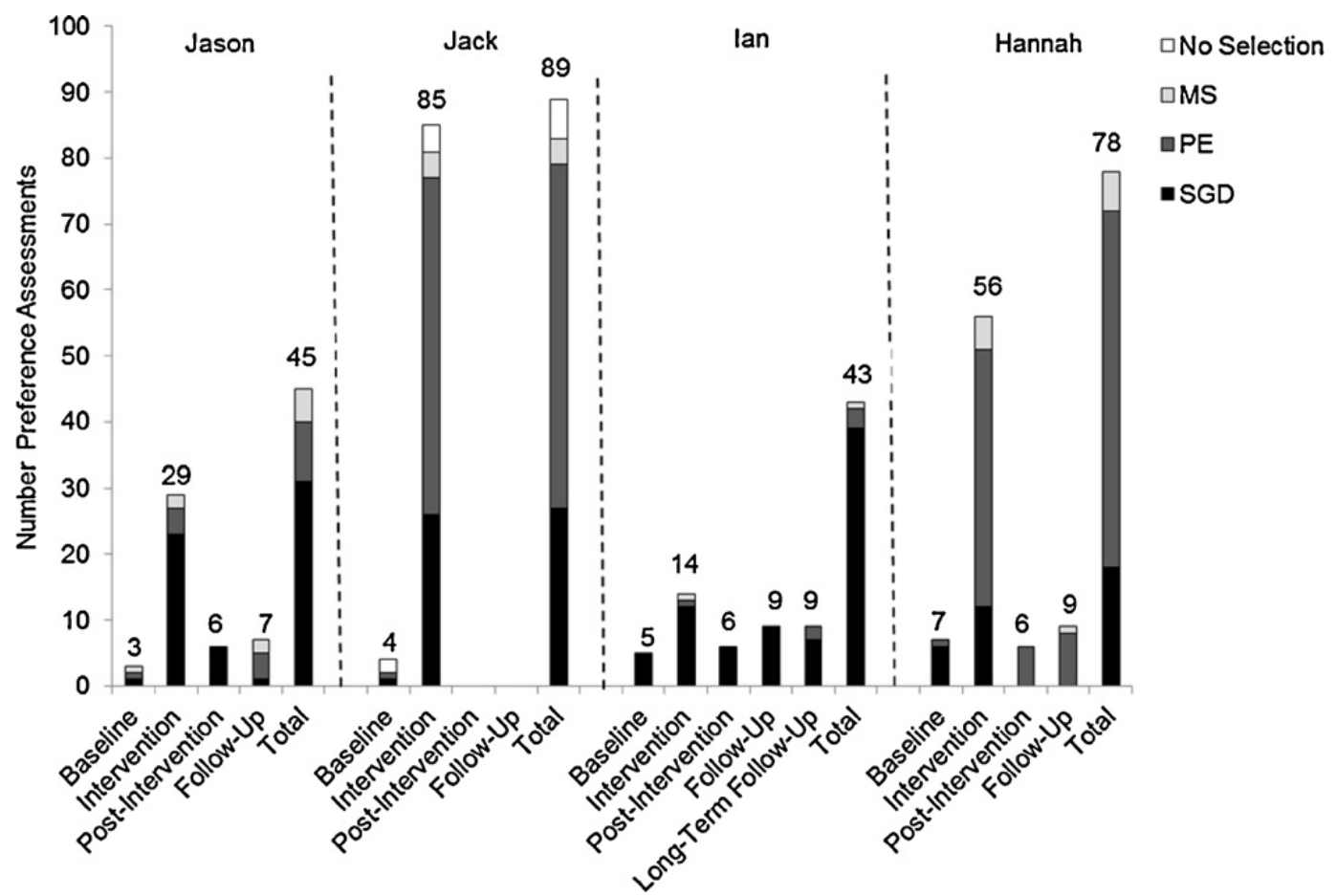

Fig. 2. Results from the AAC preference assessments depicting the number of times each communication option (SGD, PE, and MS) was chosen across phases and participants. 
intervention, post-intervention, and follow-up phases, Ian received 43 AAC preference assessments (Fig. 2) during which he chose the SGD the majority of the time (91\%).

\subsection{Hannah}

Hannah achieved acquisition criterion for PE- and SGD-based requests on her fourth and eighth respective intervention sessions (Fig. 1). She showed steady increases in the percentage of correct requests using MS, and finally reached criterion on her 34th MS intervention session (Fig. 1). During the post-intervention phase, Hannah always chose to use PE and then used it with 100\% proficiency. During follow-up, SGD and PE use maintained at high levels (92-100\%), while MS dropped to 25$33 \%$ correct requesting. Hannah did not receive pre-baseline AAC preference assessments. During baseline she chose the SGD $68 \%$ of the time, but overall across the total of 78 AAC preference assessments conducted during the study (Fig. 2 ), she chose PE most frequently (69\%).

\section{Discussion}

The first aim/hypothesis of this study was to determine if children with ASD could learn to request preferred items using one of three different AAC systems. This aim/hypothesis was achieved/confirmed with all four participants learning to make specific requests for preferred items using at least one of the three AAC modes taught. Specifically, two participants (Ian and Hannah) reached criterion with all three communication modes, while one participant reached acquisition criterion with the SGD and one (Jack) learned to use PE with moderate proficiency. These findings are consistent with previous studies reporting that children with ASD can learn to use SGDs, PE, and MS to make requests for preferred items (Flippin et al., 2010; van der Meer \& Rispoli, 2010; Wendt, 2009). Furthermore, van der Meer, Didden, et al. (2012) and van der Meer, Kagohara et al. (2012) showed that eight children with developmental disabilities learned to use SGD, PE, and MS to make general requests for snacks or toys. The present results extend the previous research by van der Meer and colleagues by demonstrating that four new children with ASD could concurrently learn to use SGD, PE, and MS to make specific requests.

The present results also extend previous research by showing that parents (i.e., Jason, Jack, and Ian's mothers) and a teaching assistant (for Hannah) could effectively apply the intervention procedures. Given that parents and teaching staff are arguably the children's more important communicative partners, this extension increases the applied relevance of the present findings, relative to van der Meer, Didden, et al. (2012) and van der Meer, Kagohara, et al. (2012), in which research staff implemented the procedures. The strategies for training these parents and the teaching assistant appeared to have been effective as evidenced by (a) the generally positive results with respect to acquisition of MS-, PE-, and SGD-based requesting, and (b) the high degree of procedural integrity. The fact that parents and the teaching assistant learned to effectively implement the procedures with relatively little training is not surprising given that our strategies for doing so were consistent with best practices for training paraprofessionals to implement similar types of behaviorally oriented teaching programs (Reid, O’Kane, \& Macurik, 2011).

The findings also demonstrated variability with which children with ASD learned the three AAC modes in line with the findings of van der Meer, Didden, et al. (2012) and van der Meer, Kagohara, et al. (2012). For example, one participant (Jack) did not learn to use MS and the remaining participants mastered MS at a slower rate than the other communication modes. van der Meer, Didden, et al. (2012) provide three possible reasons for this finding: (a) it may be more difficult to learn MS because it requires recall memory whereas graphic symbols (used for SGD and PE) could be less demanding on children's working memory - only needing recognition memory (Iacono \& Duncum, 1995; Iacono et al., 1993), (b) MS may be a more difficult AAC system to teach because forming signs requires more physical motions than pointing to or handing over a graphic symbol to a communication partner (SGD and PE), or (c) MS was the least preferred option, which may have reduced motivation to use it.

The second and third aims/hypotheses were to examine if children would indicate a preference for using one AAC system over the others during the early stages of acquisition and whether any such preferences would be stable over time. The initial choice-making patterns demonstrated by three of the participants (Jason, Ian, and Hannah) suggested a preference for using the SGD, while the remaining participant (Jack) chose each AAC system comparably often. Results suggested that for three participants (Jason, Jack, and Hannah), the pre-baseline and/or baseline choices for the different AAC systems were more variable, with preferences changing throughout later phases of the study as they learned to use each AAC mode. Therefore it could be hypothesized that some children might indicate an immediate preference based (perhaps) on some inherent features of the available AAC options (e.g., voice-output or dynamic display of SGDs), but that as they gain proficiency in using each AAC mode their preferences may change to reflect other features, such as ease of use. This could also explain lower rates for mastery and preference of MS in that the MS option might have less instant appeal. This in turn could mean that some children were less motivated to learn to use the MS. Further, the findings indicated that for some participants, their preferences for the three AAC modes only seemed to be stable once they achieved acquisition with each AAC mode. One implication of this finding is that preferences might be reliably assessed, and remain stable only after children have learned to use each communication mode to some [high] level of proficiency.

The final aim/hypothesis was to ascertain whether any demonstrated AAC system preferences would influence acquisition and maintenance of newly acquired requesting skills. Three participants (Jason, Ian, and Hannah) learned to use and/or achieved criterion with each communication system. In addition, the follow-up data suggested that maintenance was 
higher when these three children were using their most preferred AAC mode. Interestingly, although Jason demonstrated an overall preference for the SGD prior to follow-up, his preference shifted to PE during the follow-up phase and he also performed at a higher rate with PE, than with the SGD, during the follow-up phase. This suggests that for some children, their preferences for different AAC modes may change over time and influence performance. The implication of this is that there might be value in periodically reassessing children's preferences after intervention, even when they showed a preference for a particular AAC mode during acquisition and immediately after acquisition.

The participants in the study reliably discriminated between symbols used on the PE and SGD modes. This contrasts with prior research that described the difficulties children with ASD have in learning to use aided communication systems (SGD and $\mathrm{PE}$ ) due to the symbol discrimination (matching-to-sample) and scanning repertoire necessary to differentiate between and select symbols (e.g., Cannella-Malone, DeBar, \& Sigafoos, 2009; Koul, Schlosser, \& Sancibrian, 2001; Sundberg \& Sundberg, 1990; Wraikat, Sundberg, \& Michael, 1991). The use of photos of specific preferred stimuli in the current study may explain why participants relatively quickly learned to discriminate and select the correct symbol corresponding to the item being offered. However, further research is required to validate the hypothesis that photos of specific items could faciliate the acquisiton of SGD- and PE-based requesting (De Paul \& Yoder, 1986; Johnston \& Cosbey, 2012).

Activation of the iPod Touch ${ }^{\circledR}$ appeared to be problematic for Jack in that he appeared to have difficulty selecting screen icons with sufficient finesse to activate the speech output. This problem has been noted in two previous studies (CannellaMalone et al., 2009; Kagohara et al., 2010). In an attempt to rectify this problem, we introduced an iPad ${ }^{\circledR}$. As anticipated, the larger display appeared to be helpful and Jack's percentage of correct SGD-based requests increased. Massed-practice trials were also introduced in an attempt to teach Jack the MS-based request. However, he only made minimal progress in learning to make the sign for LOLLY. To some extent, his relatively limited progress with all three communication modes appeared to be a motivational issue in that he did not consistently select and then play with or eat the presumed preferred stimuli. Even after a second reinforcer assessment, and subsequent introduction of new preferred stimuli Jack seemed to quickly lose interest in the items he was being taught to request. The implication here is that progress in teaching AAC-based requesting to some children, regardless of mode, is likely to be compromised in the absence of powerful reinforcers.

While the overall findings were generally positive and consistent with previous research, several limitations must be considered when interpreting the present data set. First, only four children participated in this study and thus care should be taken when considering the implications of our findings with other children. However, when these results are combined with the two related studies (van der Meer, Didden, et al., 2012; van der Meer, Kagohara, et al., 2012), there are now a total of 12 children with developmental disabilities who have shown largely similar results. Still, further research involving increased numbers of children is needed to extend the generality of the findings. Second, no data were obtained on generalization of the requesting skills taught during the study to other preferred items, setting, or communicative partners. Research investigating generalization across the three AAC modes would be an obvious future direction. In addition, the present study focused only on teaching requesting or mands. It would be instructive to consider the use of MS, PE, and SGDs when teaching other communicative functions, such as tacting and intraverbal behavior (Skinner, 1957). Third, further research should examine factors that might influence a child's preference for using one AAC system over others. This is particularly relevant considering that our findings suggest that such preferences can influence performance during intervention and follow-up, a finding that was also noted by Ringdahl et al. (2009).

In summary, our results support previous findings that children with ASD and other developmental disabilities can be taught to use three common AAC systems to make requests and will also often show a preference for using one AAC system over others. The results extend the previous studies by van der Meer, Didden, et al. (2012), van der Meer, Kagohara, et al. (2012) by showing that while AAC mode preferences may be present prior to intervention, they can often change during and after the intervention phase. In addition, the present results provide further evidence to suggest that acquisition of AACbased requesting is faster and maintenance is better when children use their more preferred AAC mode. The results thus provide more evidence to support the value of assessing children's preferences for different AAC modes when designing and implementing communication interventions.

\section{Conflicts of interests}

The authors report no conflicts of interests and are solely responsible for the content and writing of this paper.

\section{Acknowledgements}

Preparation of this manuscript was supported in part by a grant from the New Zealand Government through the Marsden Fund Council, administered by the Royal Society of New Zealand; and by Victoria University of Wellington, The University of Canterbury, and The New Zealand Institute of Language, Brain \& Behaviour.

\section{References}

Adkins, T., \& Axelrod, S. (2001). Topography- versus selection-based responding: Comparison of mand acquisition in each modality. The Behavior Analyst Today, 2, $259-266$.

Beck, A., Stoner, J., Bock, S., \& Parton, T. (2008). Comparison of PECS and the use of a VOCA: A replication. Education and Training in Developmental Disabilities, 43, 198-216. 
Beukelman, D., \& Mirenda, P. (2005). Augmentative and alternative communication: Supporting children and adults with complex communication needs (3rd ed.). Baltimore: Paul H Brookes Publishing Co.

Bock, S., Stoner, J., Beck, A., Hanley, L., \& Prochnow, J. (2005). Increasing functional communication in non-speaking preschool children: Comparison of PECS and VOCA. Education and Training in Developmental Disabilities, 40, 268-278.

Bondy, A., \& Frost, L. (2009). The picture exchange communication system: Clinical and research applications. In P. Mirenda \& T. Iacono (Eds.), Autism spectrum disorders and AAC (pp. 279-302). Baltimore: Paul H. Brookes Publishing Co.

Cannella-Malone, H., DeBar, R., \& Sigafoos, J. (2009). An examination of preference for augmentative and alternative communication devices with two boys with significant intellectual disabilities. Augmentative and Alternative Communication, 25, 262-273.

DeLeon, I., \& Iwata, B. (1996). Evaluation of a multiple-stimulus presentation format for assessing reinforcer preferences. Journal of Applied Behavior Analysis, 29, $519-532$.

De Paul, R., \& Yoder, D. (1986). Iconicity in manual sign systems for the augmentative communication user: Is that all there is? Augmentative and Alternative Communication, 2, 1-10.

Duker, P., Didden, R., \& Sigafoos, J. (2004). One-to-one training: Instructional procedures for learners with developmental disabilities. Austin: Pro-Ed.

Flippin, M., Reszka, S., \& Watson, L. (2010). Effectiveness of the picture exchange communication system (PECS) on communication and speech for children with autism spectrum disorders: A meta-analysis. American Journal of Speech-Language Pathology, 19, 178-195.

Green, V. A., Sigafoos, J., Didden, R., O’Reilly, M. F., Lancioni, G. E., Ollington, N., \& Payne, D. (2008). Validity of a structured interview protocol for assessing children's preferences. In P. Grotewell \& Y. Burton (Eds.), Early childhood education: Issues and developments (pp. 87-103). New York: Nova Science Publishers.

Gregory, M., DeLeon, I., \& Richman, D. (2009). The influence of matching and motor-imitation abilities on rapid acquisition of manual signs and exchange-based communication responses. Journal of Applied Behavior Analysis, 42, 399-404.

Iacono, T., \& Duncum, J. (1995). Comparison of sign alone and in combination with an electronic communication device in early language intervention: Case study. Augmentative and Alternative Communication, 11, 249-259.

Iacono, T., Mirenda, P., \& Beukelman, D. (1993). Comparison of unimodal and multimodal AAC techniques for children with intellectual disabilities. Augmentative and Alternative Communication, 9, 83-94.

Johnston, S., \& Cosbey, J. (2012). Building blocks of a beginning communication system: Communicative modes. In S. Johnston, J. Reichle, K. Feeley, \& E. Jones (Eds.), AAC strategies for individuals with moderate to severe disabilities (pp. 25-50). Baltimore: Paul H. Brookes Publishing Co.

Kagohara, D., van der Meer, L., Achmadi, D., Green, V. A., O’Reilly, M. F., Mulloy, A., \& Sigafoos, J. (2010). Behavioral intervention promotes successful use of an iPodbased communication device by an adolescent with autism. Clinical Case Studies, 9, 328-338.

Kennedy, C. (2005). Single-case designs for educational research. Boston: Pearson.

Koul, R., Schlosser, R. W., \& Sancibrian, S. (2001). Effects of symbol, referent and instructional variables on the acquisition of aided and unaided symbols by individuals with autism spectrum disorders. Focus on Autism E' Other Developmental Disabilities, 16, 162-169.

Lancioni, G. E., O'Reilly, M. F., Cuvo, A., Singh, N. N., Sigafoos, J., \& Didden, R. (2007). PECS and VOCAs to enable students with developmental disabilities to make requests: An overview of the literature. Research in Developmental Disabilities, 28, 468-488.

Makaton New Zealand/Aotearoa. (1998-1999). Sign illustrations for Makaton core vocabulary. Auckland: Westprint.

Matson, J. L., Mahan, S., Kozlowski, A. M., \& Shoemaker, M. (2010). Developmental milestones in toddlers with autistic disorder, pervasive developmental disorder-not otherwise specified and atypical development. Developmental Neurorehabilitation, 13, $239-247$.

Mirenda, P. (2003). Toward functional augmentative and alternative communication for students with autism: Manual signs, graphic symbols, and voice output communication aids. Language, Speech, E Hearing Services in Schools, 34, 203-216.

Reid, D. H., O'Kane, N. P., \& Macurik, K. M. (2011). Staff training and management. In W. Fisher, C. C. Piazza, \& H. S. Roane (Eds.), Handbook of applied behavior analysis (pp. 281-294). New York: Guilford Press.

Ringdahl, J., Falcomata, T., Christensen, T., Bass-Ringdahl, S., Lentz, A., Dutt, A., \& Schuh-Claus, J. (2009). Evaluation of a pre-treatment assessment to select mand topographies for functional communication training. Research in Developmental Disabilities, 20, 330-341.

Schlosser, R. W. (2003). The efficacy of augmentative and alternative communication: Toward evidence-based practice. San Diego: Academic Press.

Schlosser, R. W., \& Blischak, D. (2001). Is there a role for speech output in interventions for persons with autism? Focus on Autism E' Other Developmental Disabilities, $16,170-178$.

Sennott, S., \& Bowker, A. (2009). Autism, AAC, and Proloquo2Go. Perspectives on Augmentative and Alternative Communication, $18,137-145$.

Sigafoos, J., \& Drasgow, E. (2001). Conditional use of aided and unaided AAC: A review and clinical case demonstration. Focus on Autism \& Other Developmental Disabilities, 16, 152-161.

Skinner, B. F. (1957). Verbal behavior. Englewood Cliffs, NJ: Prentice-Hall.

Sparrow, S., Cicchetti, D., \& Balla, D. (2005). Vineland-II adaptive behavior scales (2nd ed.). Minneapolis: Pearson.

Sundberg, C., \& Sundberg, M. (1990). Comparing topography-based verbal behavior with stimulus selection-based verbal behavior. The Analysis of Verbal Behavior, $8,31-41$.

Tincani, M. (2004). Comparing the Picture Exchange Communication System and sign language training for children with autism. Focus on Autism E Other Developmental Disabilities, 19, 152-163.

van der Meer, L., Didden, R., Sutherland, D., O’Reilly, M., Lancioni, G., \& Sigafoos, J. (2012). Comparing three augmentative and alternative communication modes for children with developmental disabilities. Journal of Developmental and Physical Disabilities http://dx.doi.org/10.1007/s10882-012-9283-3.

van der Meer, L., Kagohara, D., Achmadi, D., O’Reilly, M., Lancioni, G., Sutherland, D., \& Sigafoos, J. (2012). Speech-generating devices versus manual signing for children with developmental disabilities. Research in Developmental Disabilities, 33, 1658-1669.

van der Meer, L., \& Rispoli, M. (2010). Communication interventions involving speech-generating devices for children with autism: A review of the literature. Developmental Neurorehabilitation, 13, 294-306.

van der Meer, L., Sigafoos, J., O'Reilly, M., \& Lancioni, G. (2011). Assessing preferences for AAC options in communication interventions for individuals with developmental disabilities: A review of the literature. Research in Developmental Disabilities, 32, 1422-1431.

Watson, P. J., \& Workman, E. A. (1981). The non-concurrent multiple baseline across-individuals design: An extension of the traditional multiple baseline design. Journal of Behavior Therapy and Experimental Psychiatry, 12, 257-259.

Wendt, O. (2009). Research on the use of manual signs and graphic symbols in autism spectrum disorders: A systematic review. In P. Mirenda \& T. Iacono (Eds.), Autism spectrum disorders and AAC (pp. 83-140). Baltimore: Paul H. Brookes Publishing Co.

Wraikat, R., Sundberg, C., \& Michael, J. (1991). Topography-based and selection-based verbal behavior: A further comparison. The Analysis of Verbal Behavior, 9, $1-17$. 